\title{
A Synthetic Carbohydrate-Protein Conjugate Vaccine Candidate against Klebsiella Pneumoniae Serotype K2
}

\author{
Mettu Ravinder ${ }^{\dagger, \perp}$, Kuo-Shiang Liao ${ }^{\dagger, \S, \perp}$, Yang-Yu Cheng ${ }^{\dagger, \S}$, Sujeet Pawar ${ }^{\dagger, \infty}$, Tzu-Lung \\ Lin $^{\#}$, Jin-Town Wang ${ }^{\#}$, Chung-Yi Wu ${ }^{\dagger, \infty, *}$
}

$\dagger$ Genomics Research Center, Academia Sinica, No. 128 Academia Road, Section 2, Nangang District, Taipei 11529, Taiwan.

§Institute of Biochemistry and Molecular Biology, National Yang-Ming University, No. 155, Sec. 2, Linong Street, Taipei, 112, Taiwan

$\infty$ Chemical Biology and Molecular Biophysics, Taiwan International Graduate Program, Academia Sinica, No. 128 Academia Road, Section 2, Nangang District, Taipei 11529, Taiwan.

\#Department of Microbiology, National Taiwan University College of Medicine, No. 1 Jen Ai Road, Section 1, Zhonzheng District, Taipei 10051, Taiwan

*To whom correspondence should be addressed: C-Y. Wu, Tel: +886-2-227871263, Fax: +886-2-27898771, Email: cyiwu@gate.sinica.edu.tw

$\perp$ These authors contributed equally. 


\section{Table of contents}

\begin{tabular}{|c|c|c|}
\hline 1 & NMR spectrum of compound $17 a$ & S1-S6 \\
\hline 2 & NMR spectrum of compound $17 \mathrm{~b}$ & S7-S11 \\
\hline 3 & NMR spectrum of compound 18 & S12-S13 \\
\hline 4 & NMR spectrum of compound 19 & S14-S18 \\
\hline 5 & NMR spectrum of compound 20 & S19-S21 \\
\hline 6 & NMR spectrum of compound 22 & S22-S24 \\
\hline 7 & NMR spectrum of compound 8 & S25-S28 \\
\hline 8 & NMR spectrum of compound 6 & S29-S35 \\
\hline 9 & NMR spectrum of compound 23 & S36-S38 \\
\hline 10 & NMR spectrum of compound 27 & S39-S41 \\
\hline 11 & NMR spectrum of compound 28 & S42-S44 \\
\hline 12 & NMR spectrum of compound 7 & S45-S47 \\
\hline 13 & NMR spectrum of compound S2b & S48-S55 \\
\hline 14 & NMR spectrum of compound S2a & S56-S64 \\
\hline 15 & NMR spectrum of compound S3 & S65-S67 \\
\hline 16 & NMR spectrum of compound S4 & S68-S76 \\
\hline 17 & NMR spectrum of compound S5 & S77-S79 \\
\hline 18 & NMR spectrum of compound S7 & S80-S83 \\
\hline 19 & NMR spectrum of compound S8 & S84-S87 \\
\hline 20 & NMR spectrum of compound S9 & S88-S94 \\
\hline 21 & NMR spectrum of compound 13 & S95-S96 \\
\hline
\end{tabular}




\section{Table of contents}

\begin{tabular}{|c|c|c|}
\hline 22 & NMR spectrum of compound 24 & S97-\$99 \\
\hline 23 & NMR spectrum of compound 14 & S100-S102 \\
\hline 24 & NMR spectrum of compound 25 & S103-S106 \\
\hline 25 & NMR spectrum of compound 15 & S107-S110 \\
\hline 26 & NMR spectrum of compound 16 & S111-S114 \\
\hline 27 & NMR spectrum of compound 29 & S115-S122 \\
\hline 28 & NMR spectrum of compound $26 a$ & S123-S127 \\
\hline 29 & NMR spectrum of compound 30 & S128-S134 \\
\hline 30 & NMR spectrum of compound 31 & S135-S142 \\
\hline 31 & $\begin{array}{l}\text { NMR and Mass spectrum of compound } 1 \\
\text { (NMR at 313k) }\end{array}$ & S143-S151 \\
\hline 32 & $\begin{array}{l}\text { NMR and Mass spectrum of compound } 2 \\
\text { (NMR at 313k) }\end{array}$ & S152-S163 \\
\hline 33 & $\begin{array}{l}\text { NMR and Mass spectrum of compound } 3 \\
\text { (NMR at 313k) }\end{array}$ & S164-S175 \\
\hline 34 & $\begin{array}{l}\text { NMR and Mass spectrum of compound } 4 \\
\text { (NMR at 313k) }\end{array}$ & S176-S186 \\
\hline 35 & $\begin{array}{l}\text { NMR and Mass spectrum of compound } 5 \\
\text { (NMR at 313k and 298k) }\end{array}$ & S187-S200 \\
\hline 36 & $\begin{array}{l}\text { NMR and Mass spectrum of compound } 3 a \\
\text { (NMR at 313k) }\end{array}$ & S201-S203 \\
\hline
\end{tabular}




\section{Table of contents}

\begin{tabular}{|c|l|c|}
\hline 37 & $\begin{array}{l}\text { NMR and Mass spectrum of compound 4a } \\
\text { (NMR at 313k) }\end{array}$ & S204-S206 \\
\hline 38 & $\begin{array}{l}\text { NMR and Mass spectrum of compound 5a } \\
\text { (NMR at 313k) }\end{array}$ & S207-S209 \\
\hline
\end{tabular}




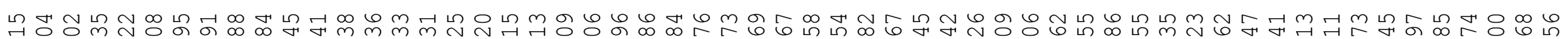

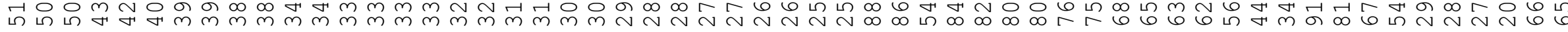

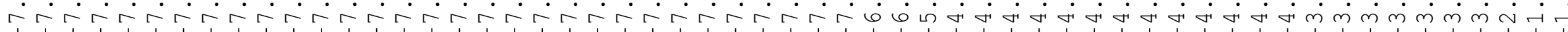

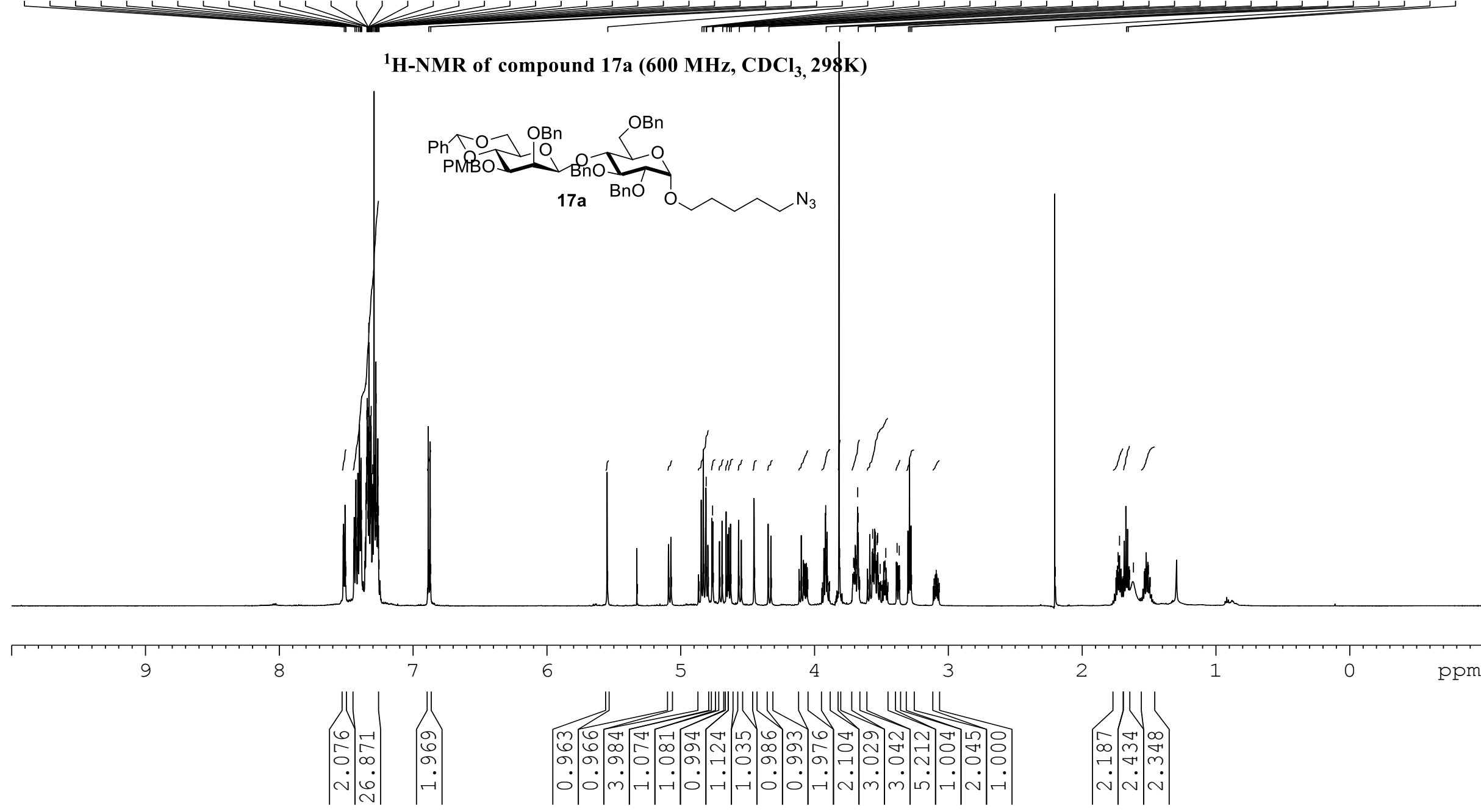




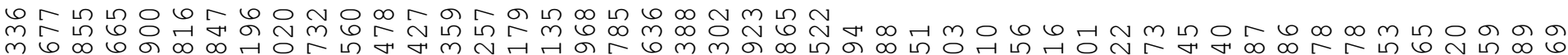
.

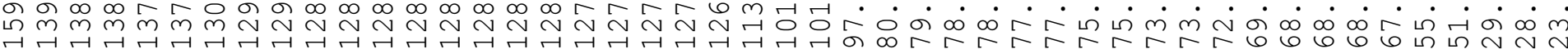

(n)

${ }^{13} \mathrm{C}-\mathrm{NMR}$ of compound $17 \mathrm{a}\left(150 \mathrm{MHz}, \mathrm{CDCl}_{3}, 298 \mathrm{~K}\right)$
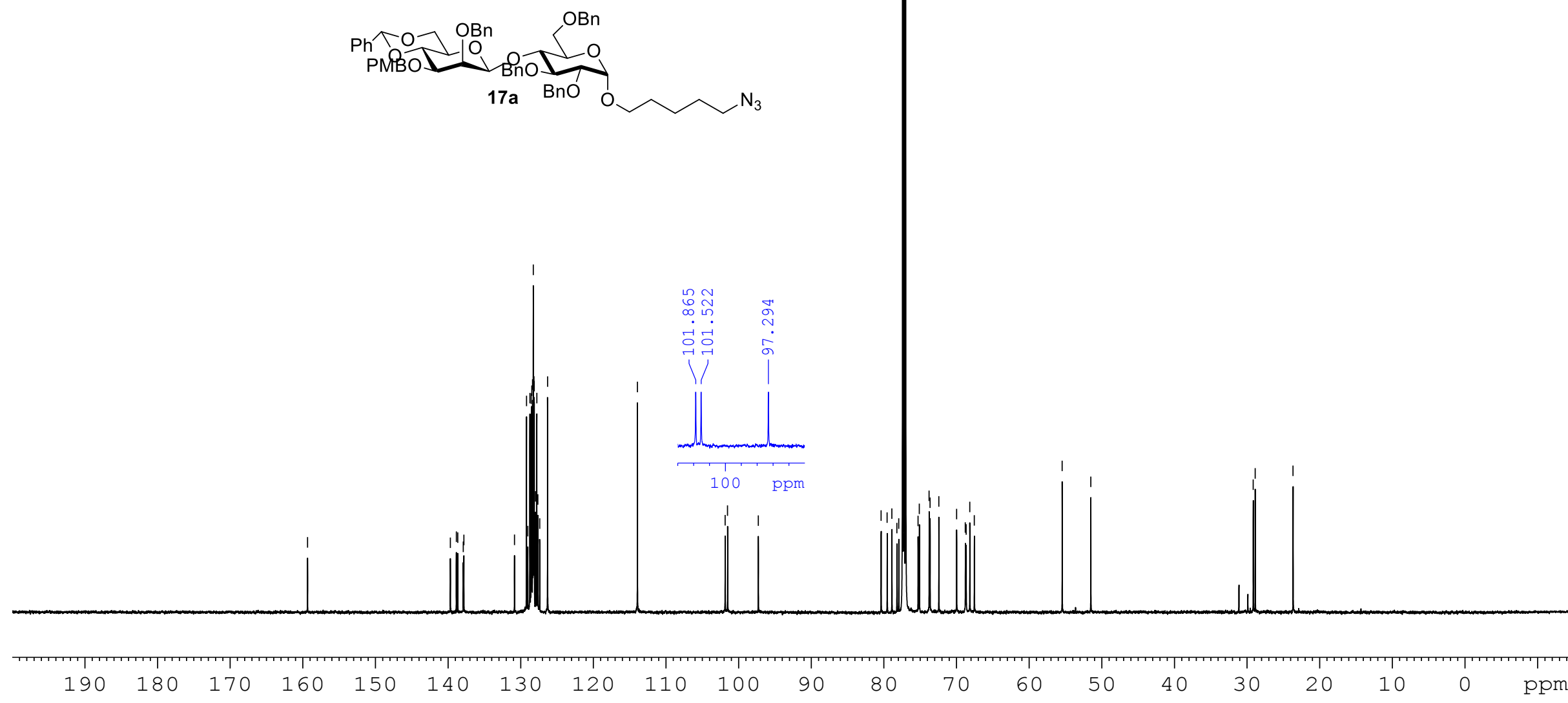


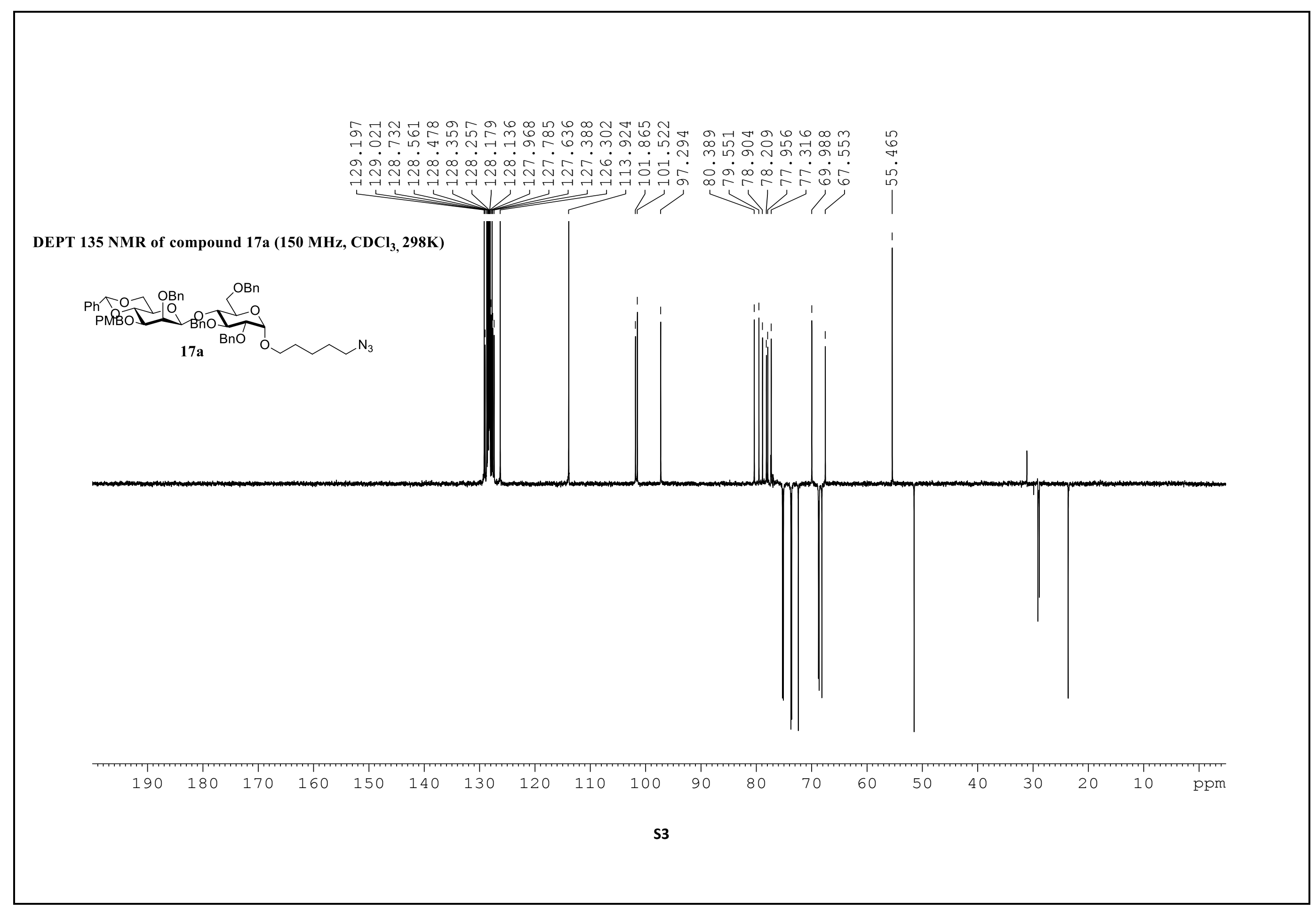




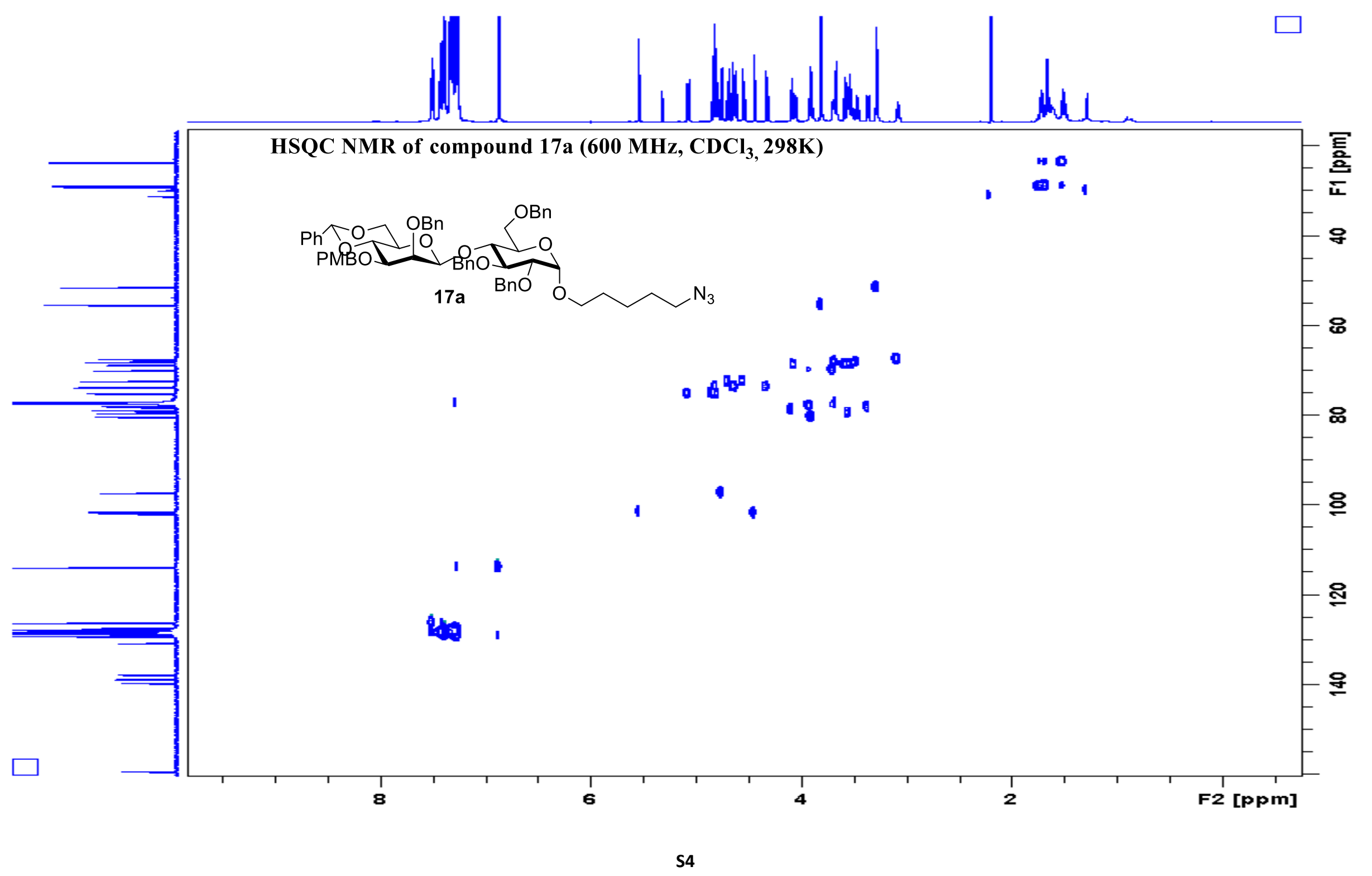




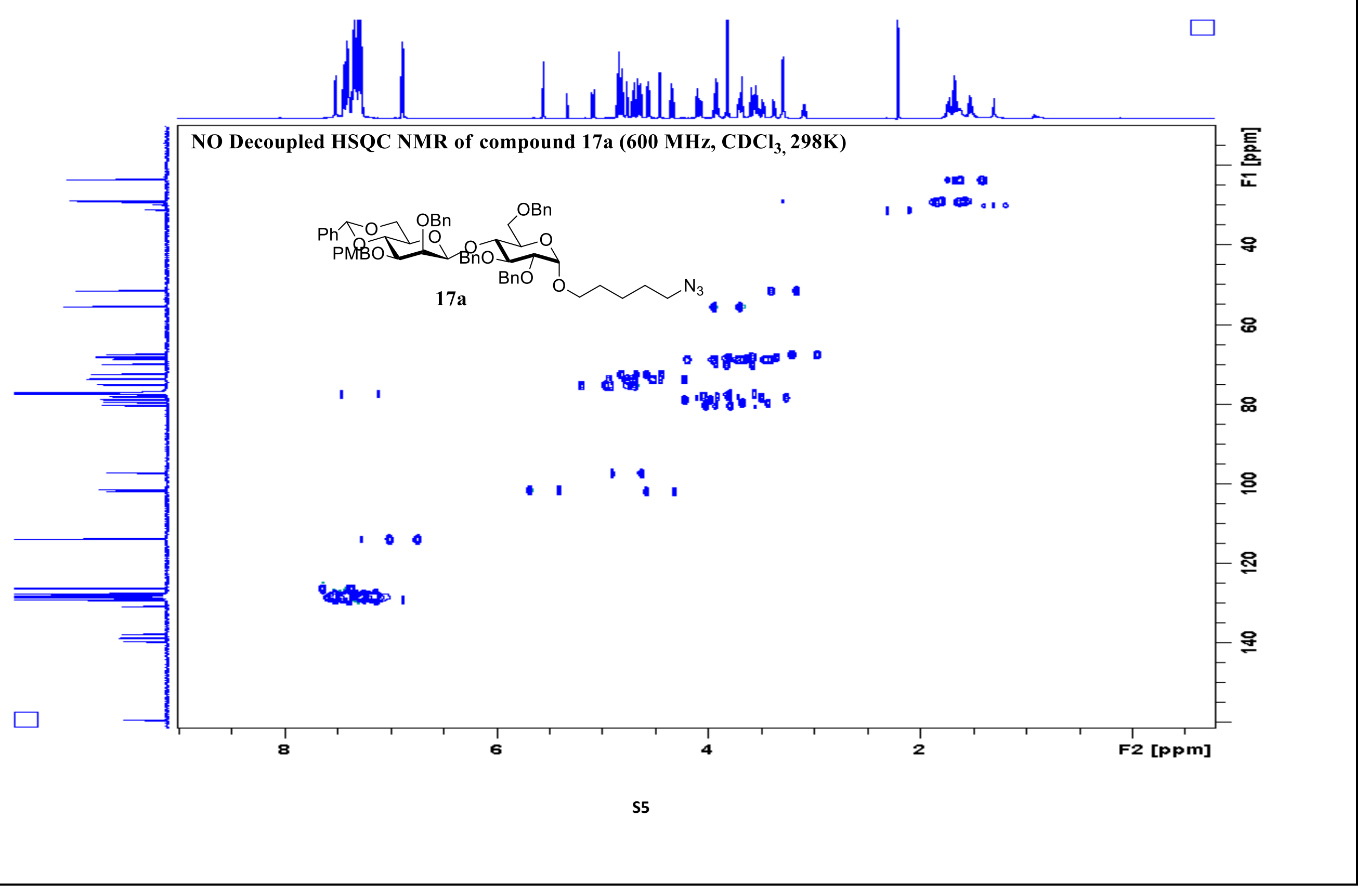




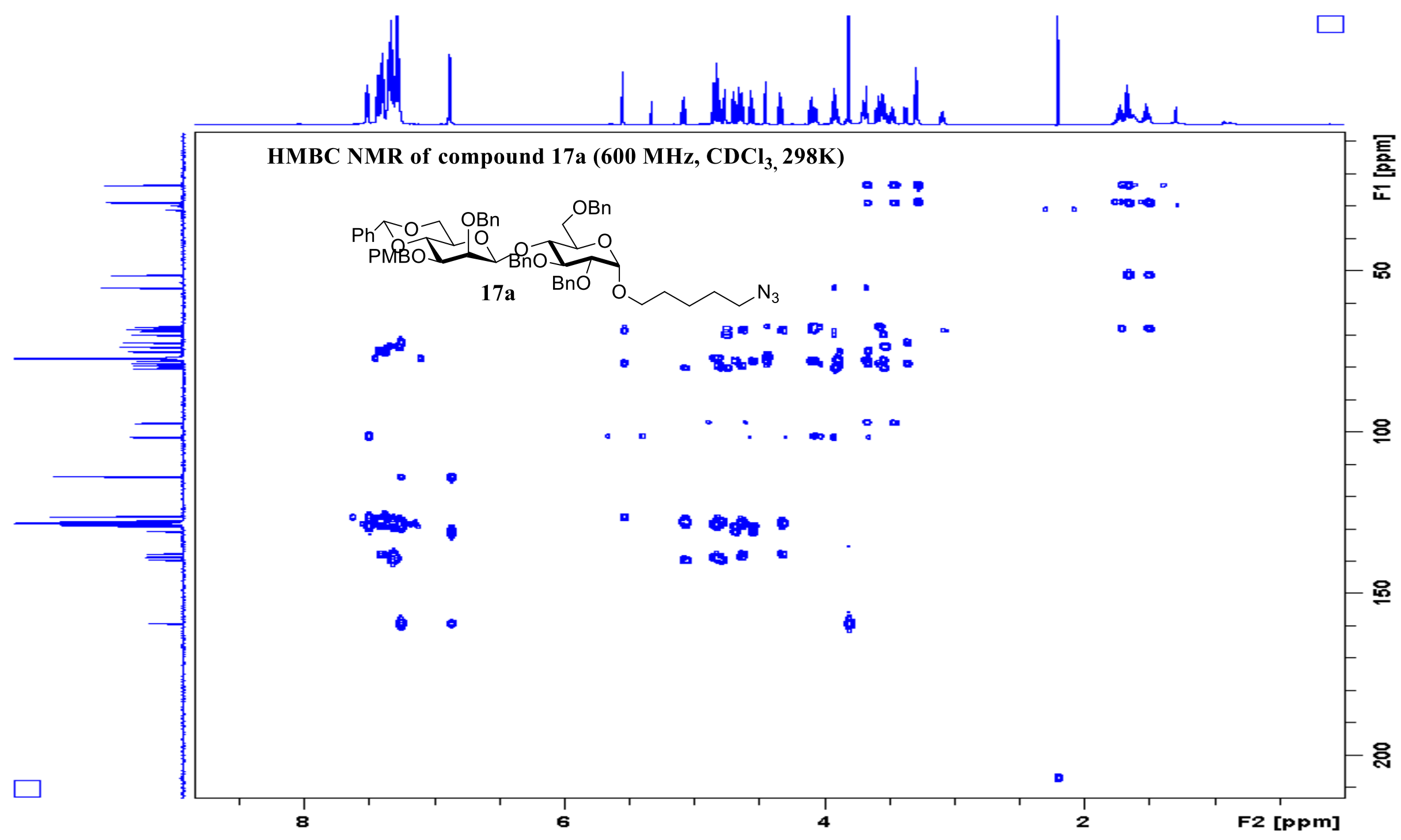




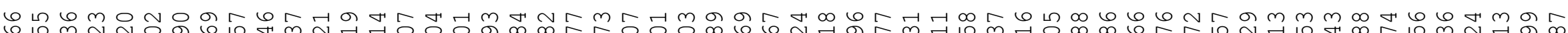

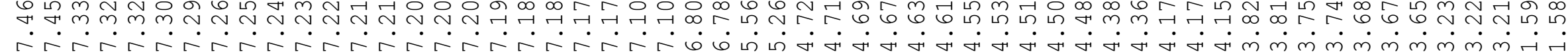

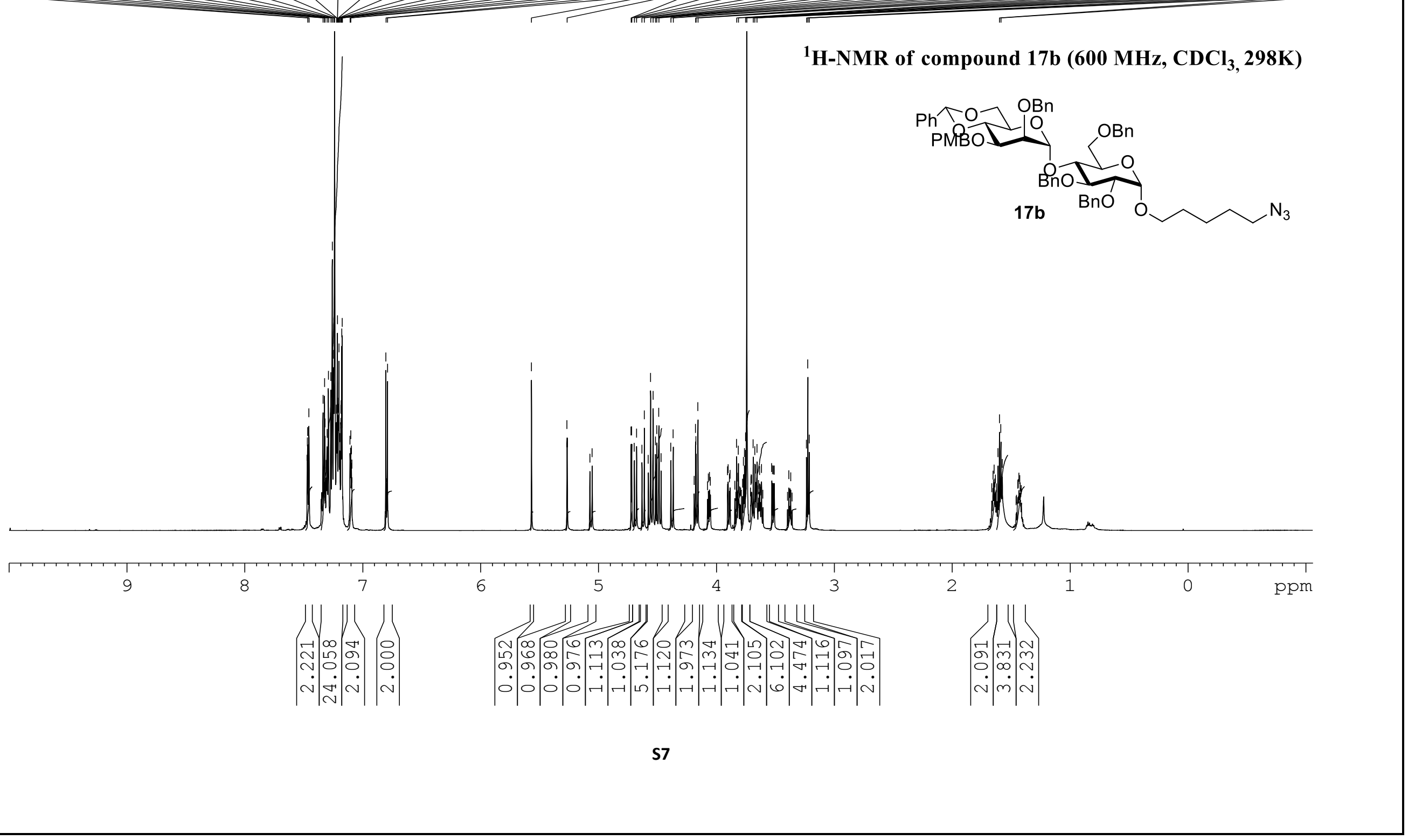




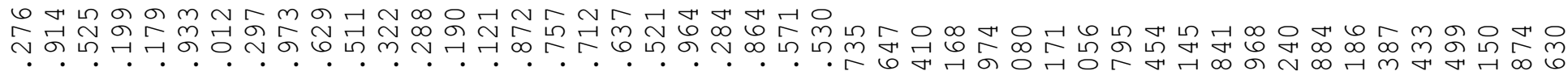

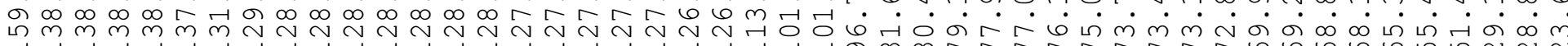
${ }_{1}^{1}$

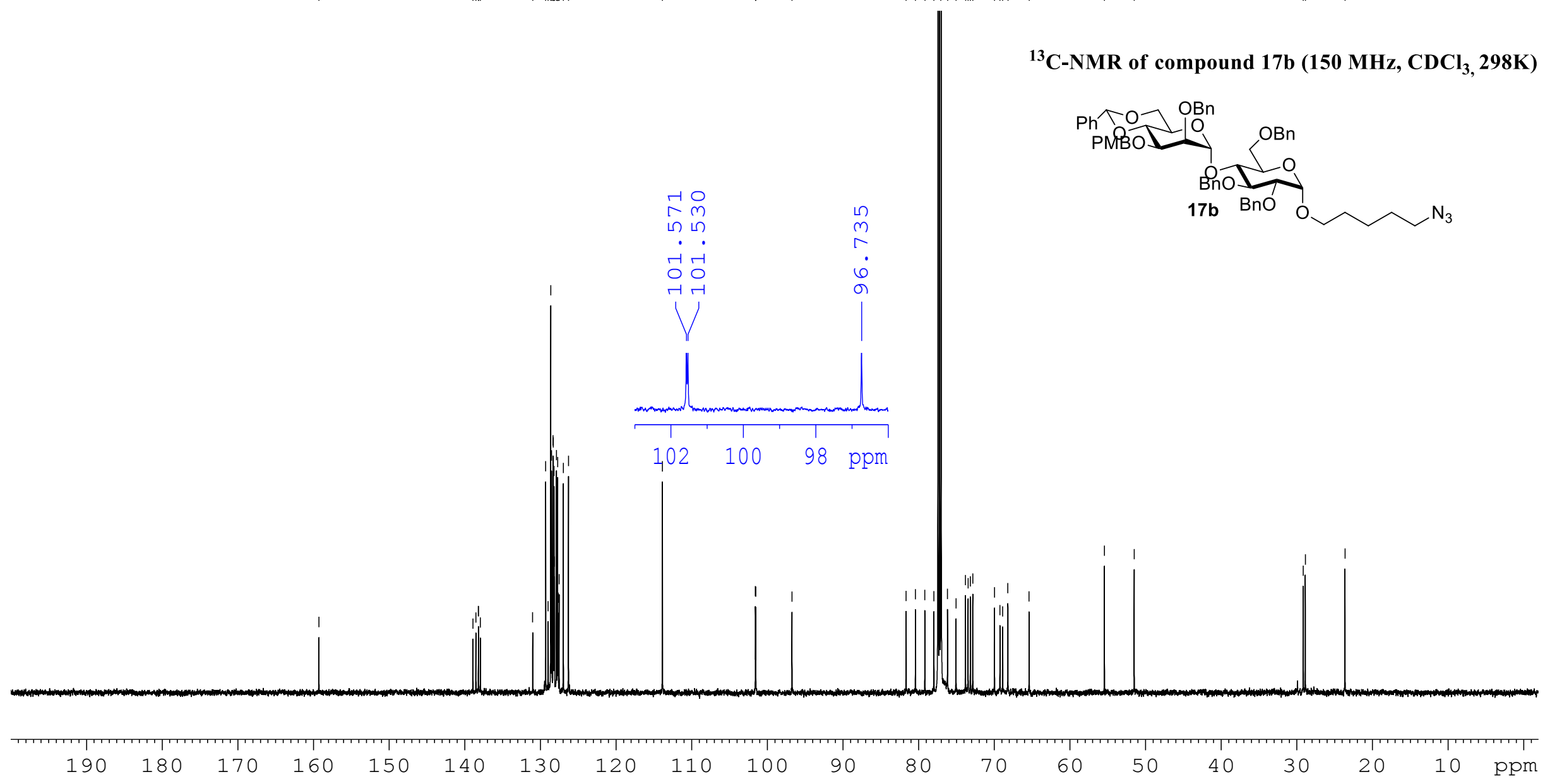




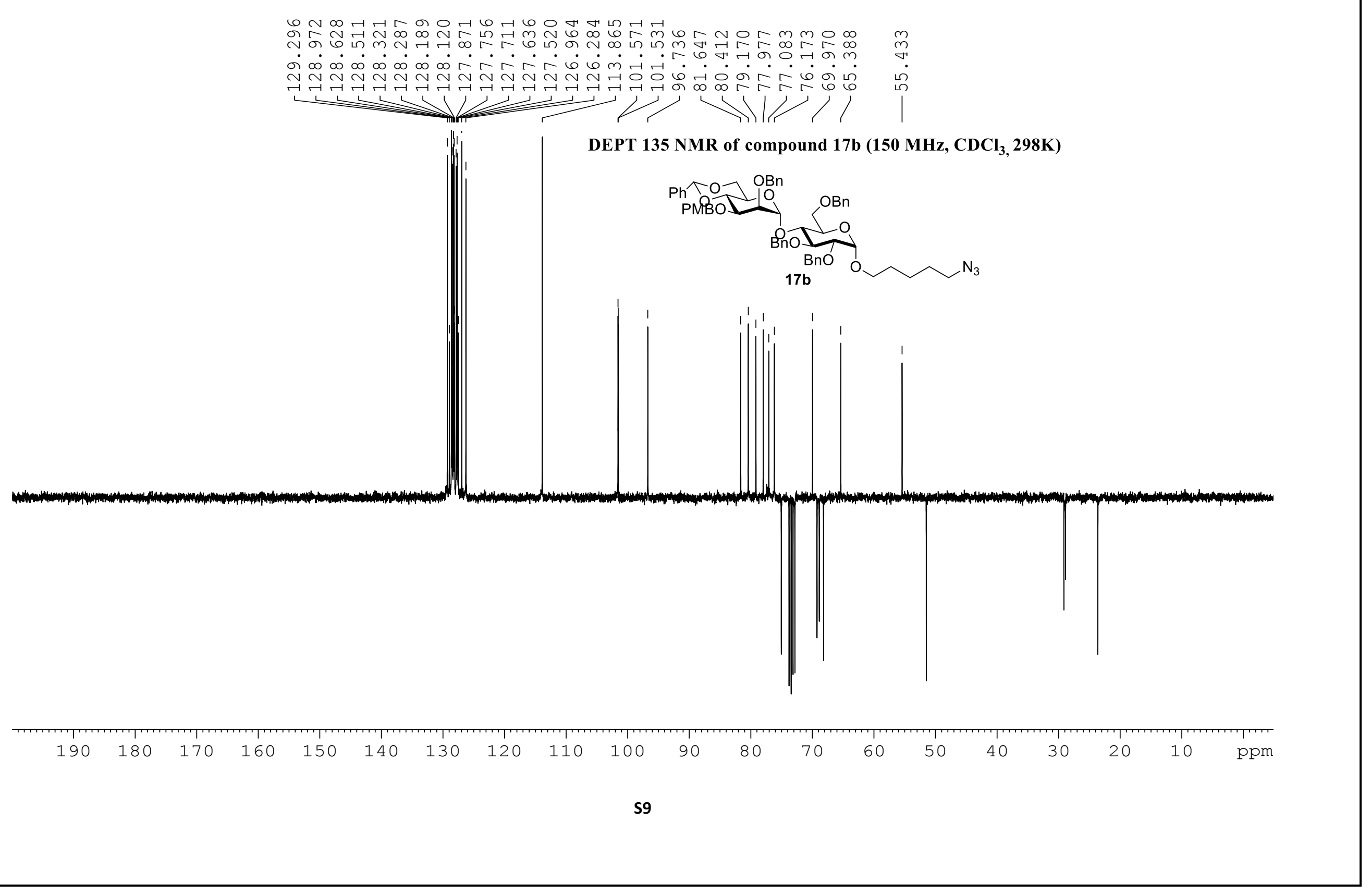




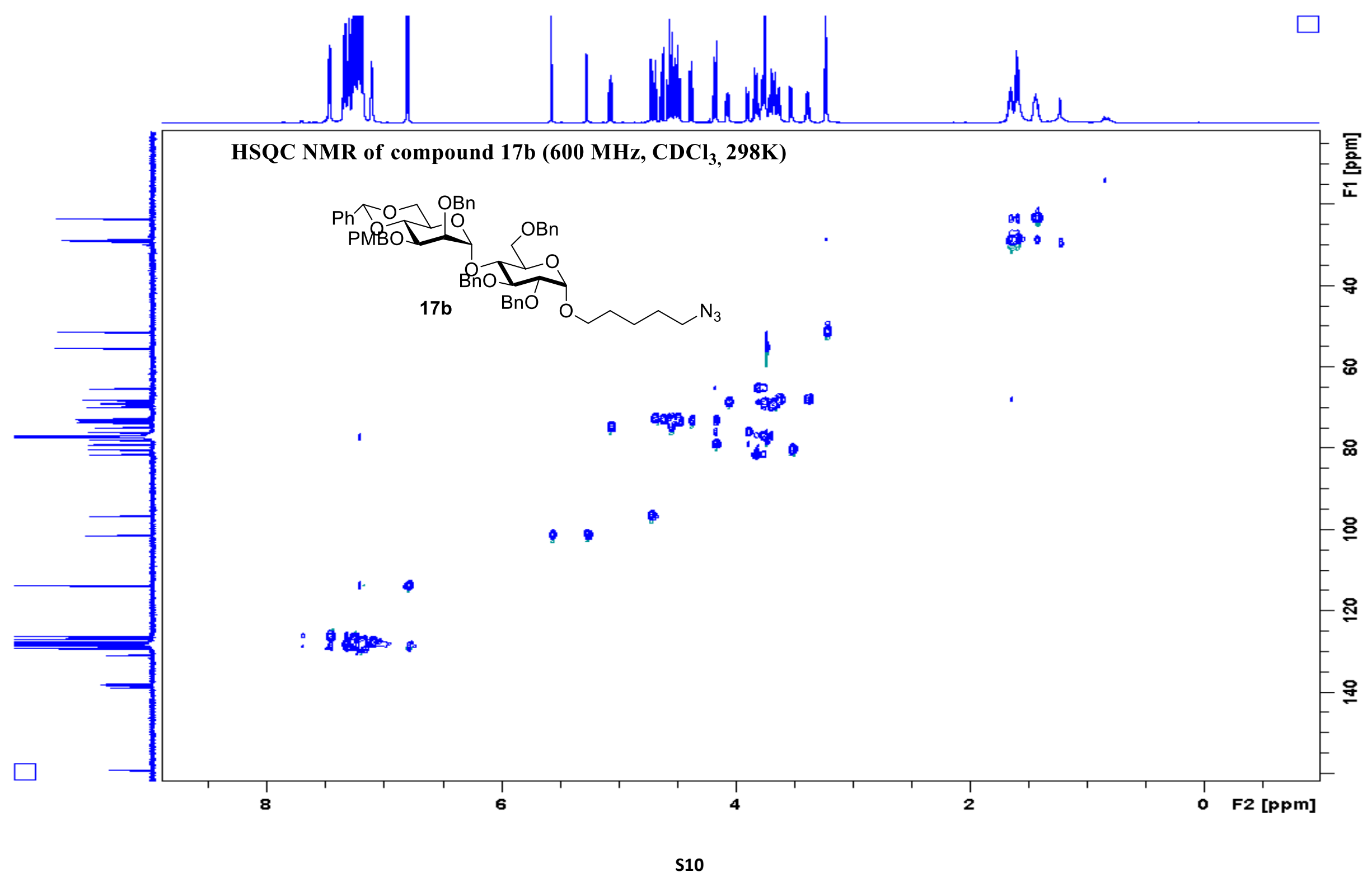




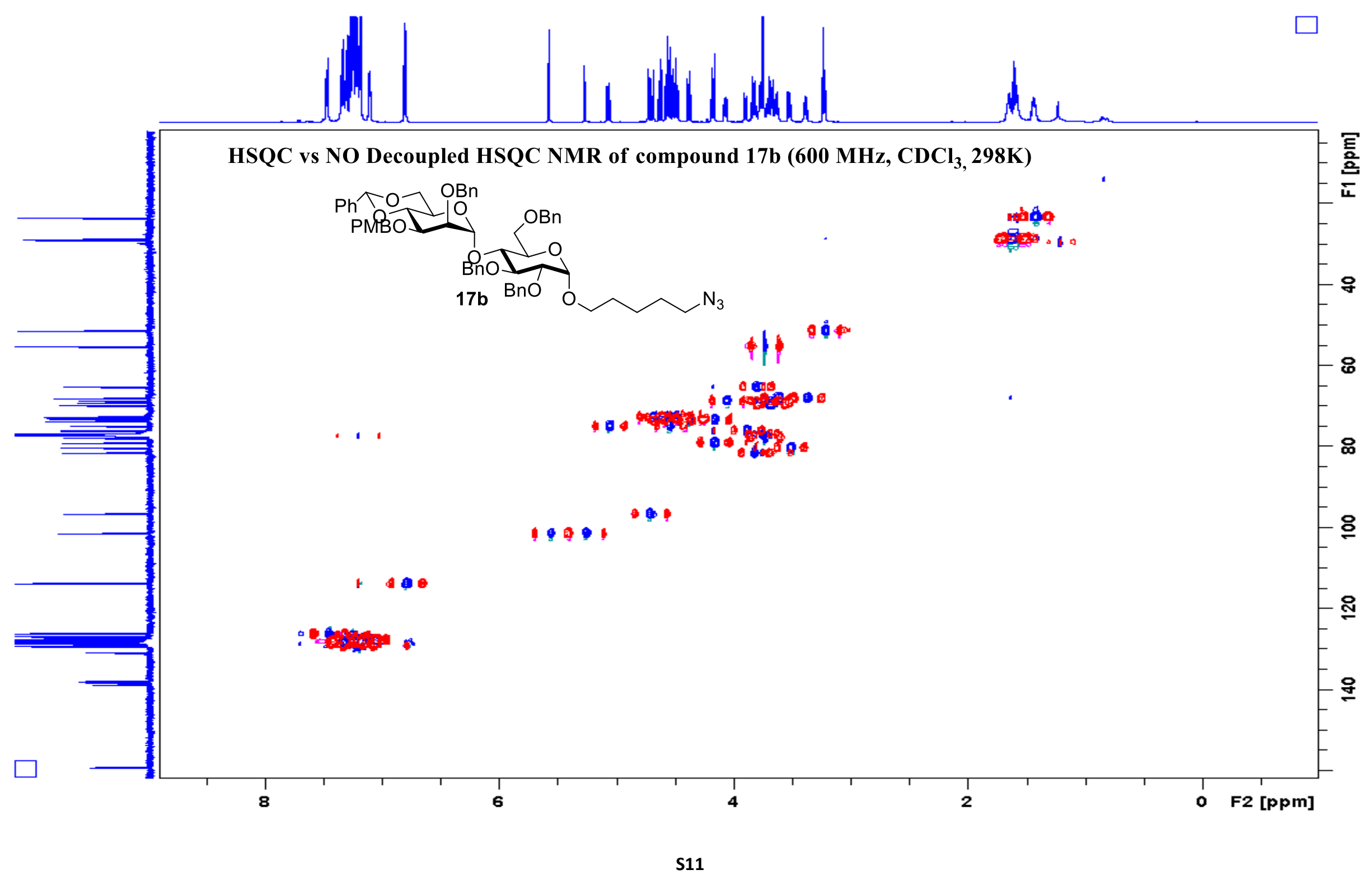




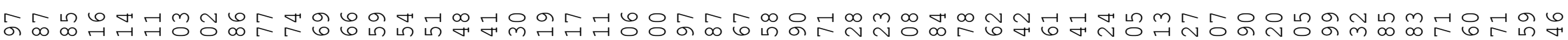

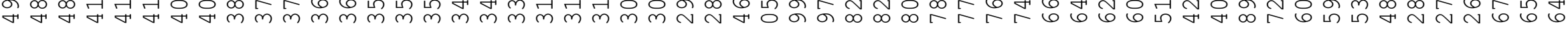

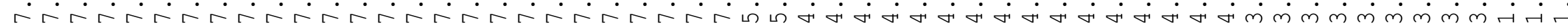

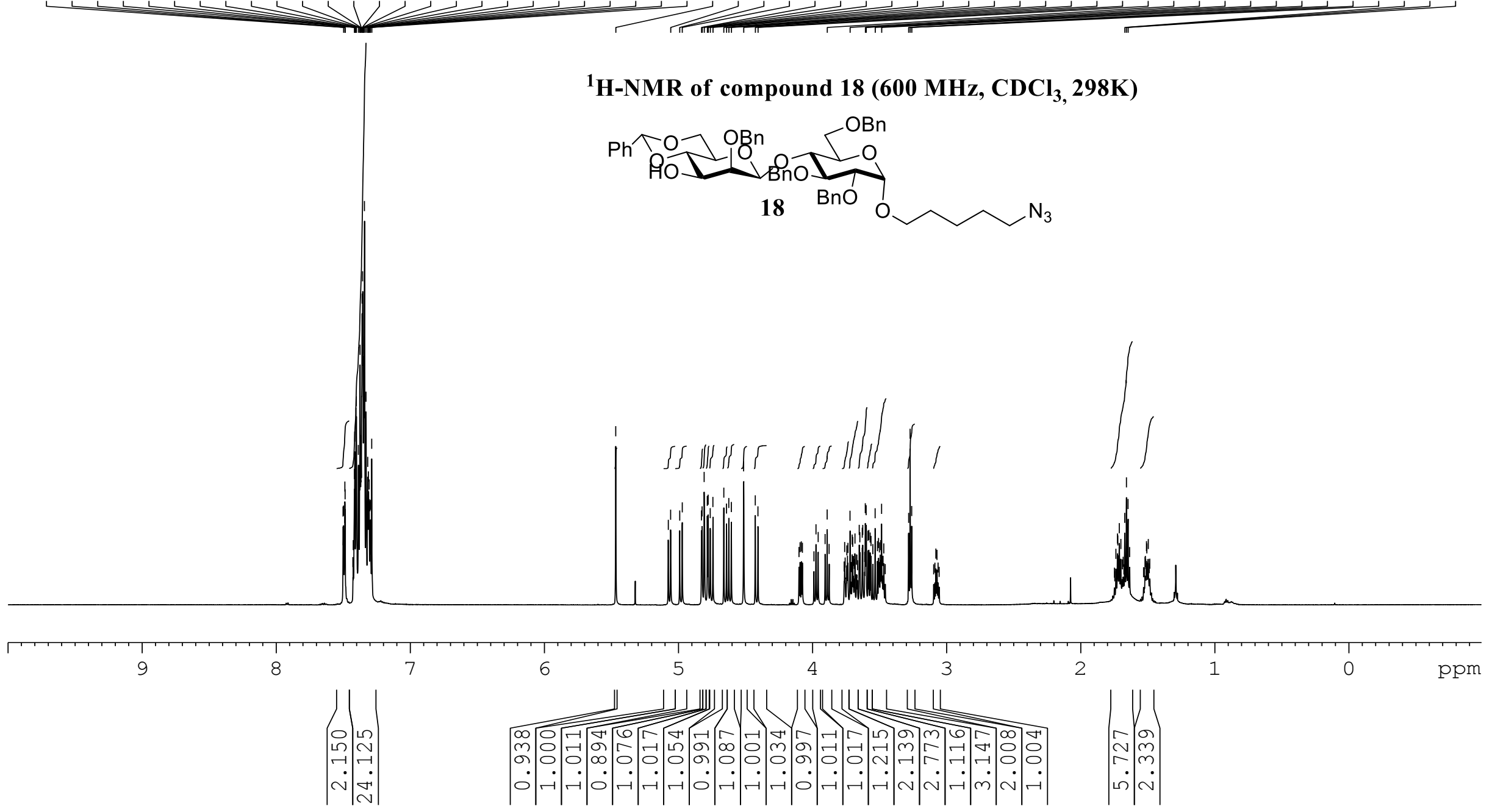




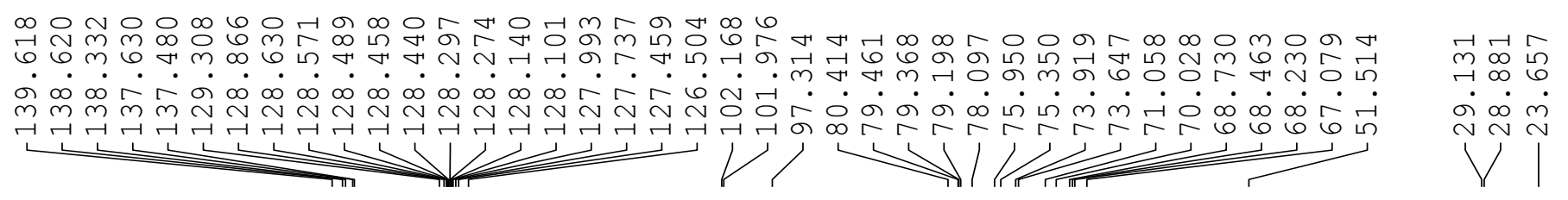

${ }^{13} \mathrm{C}$-NMR of compound $18\left(150 \mathrm{MHz}, \mathrm{CDCl}_{3}, 298 \mathrm{~K}\right)$
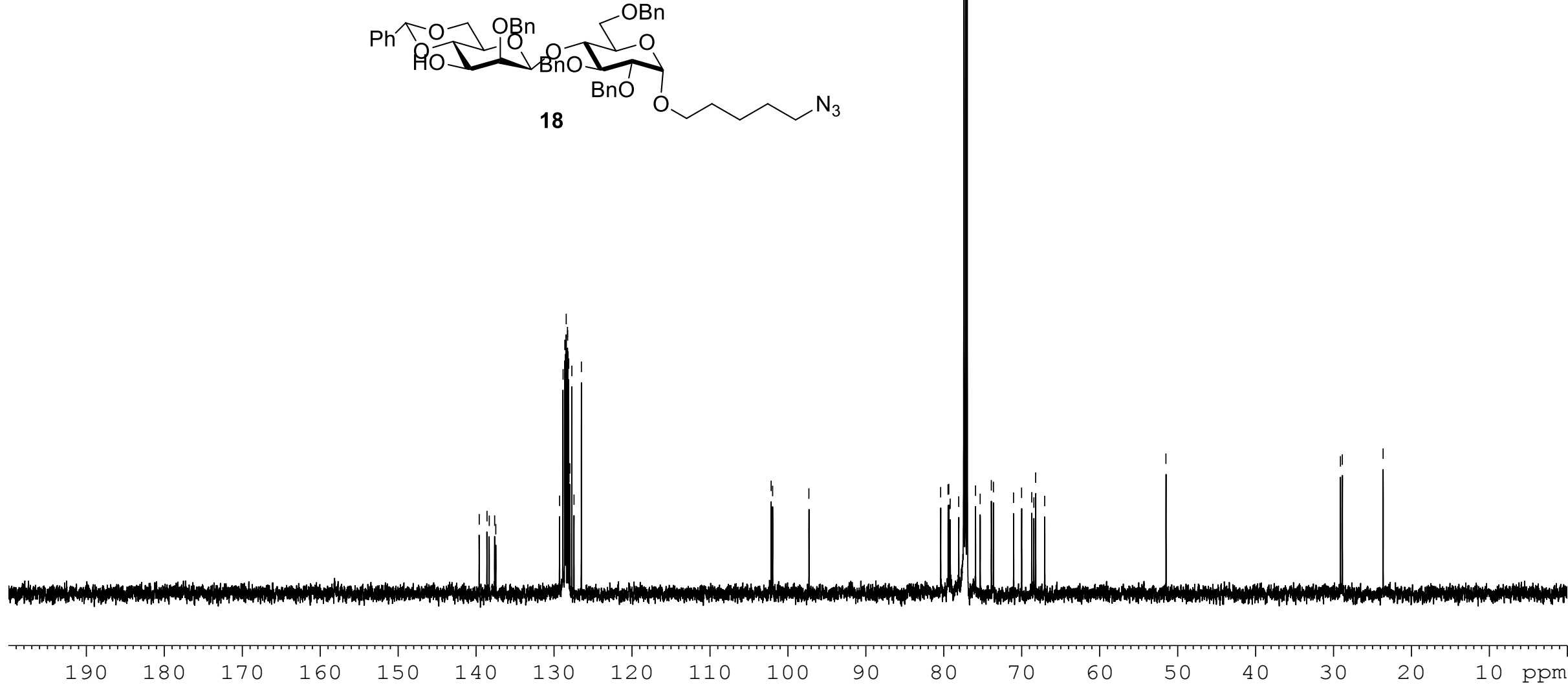


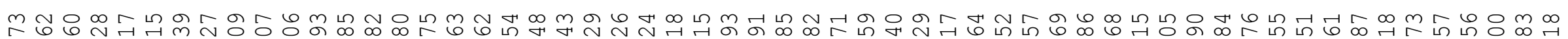

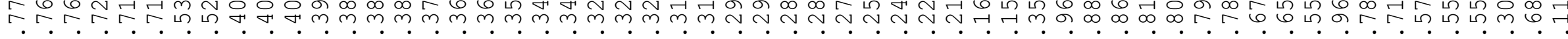

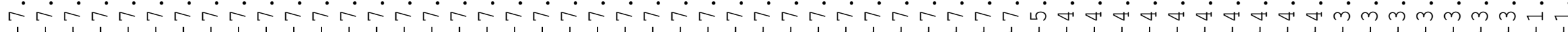
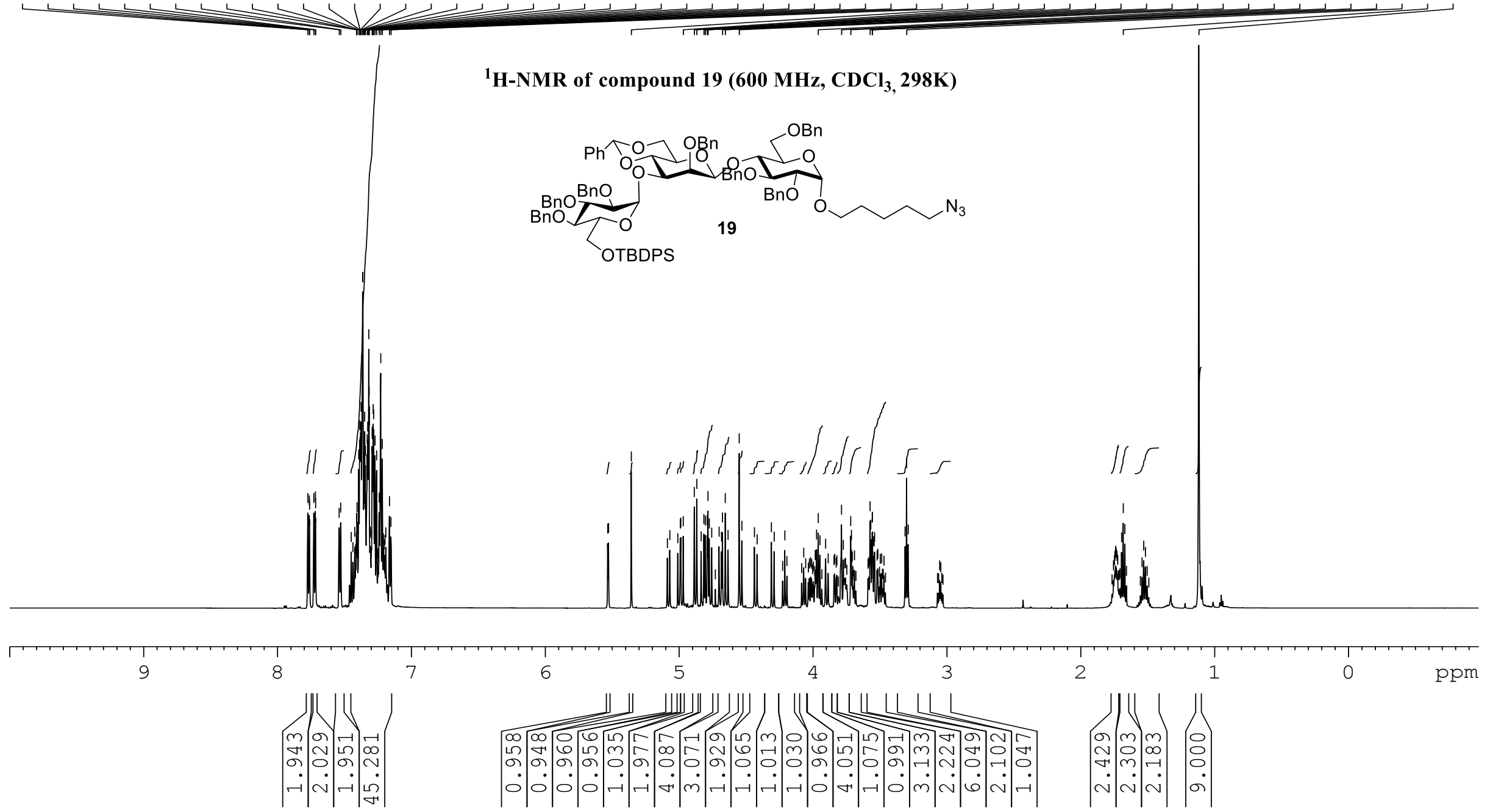


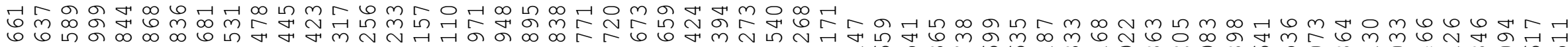

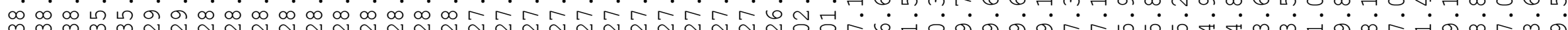

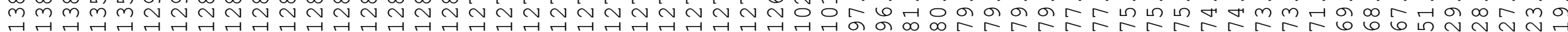

${ }^{13} \mathrm{C}-\mathrm{NMR}$ of compound $19\left(150 \mathrm{MHz}, \mathrm{CDCl}_{3}, 298 \mathrm{~K}\right)$

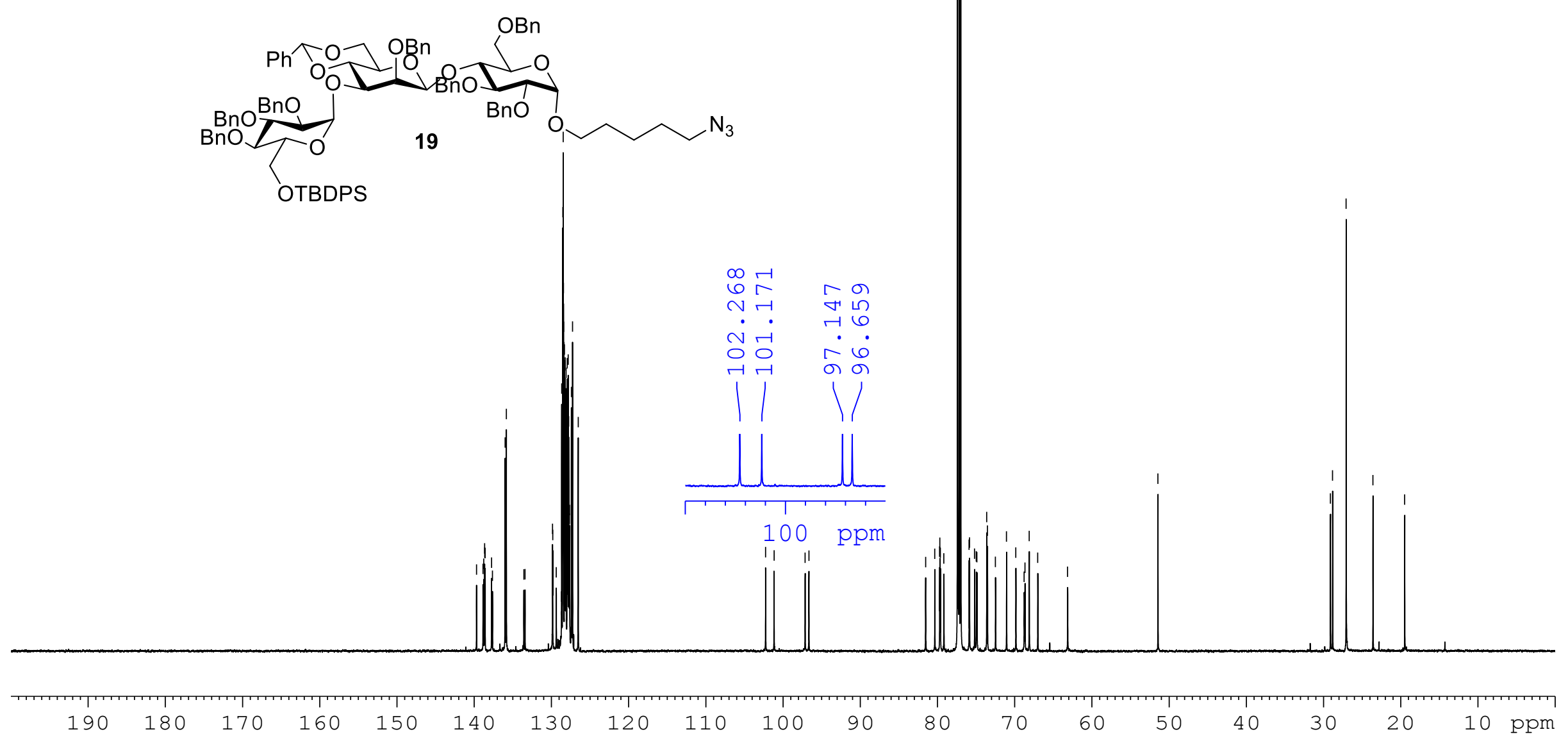




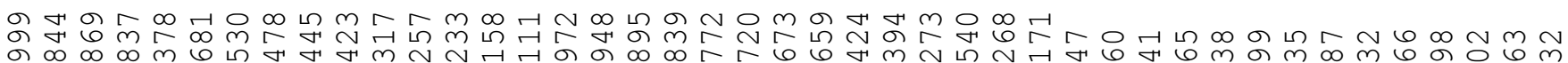

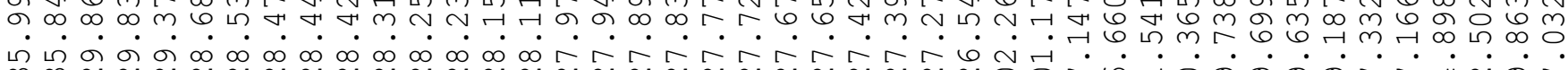

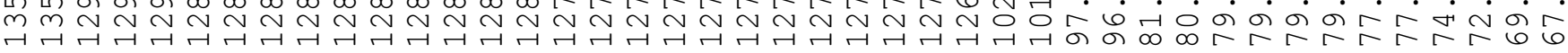

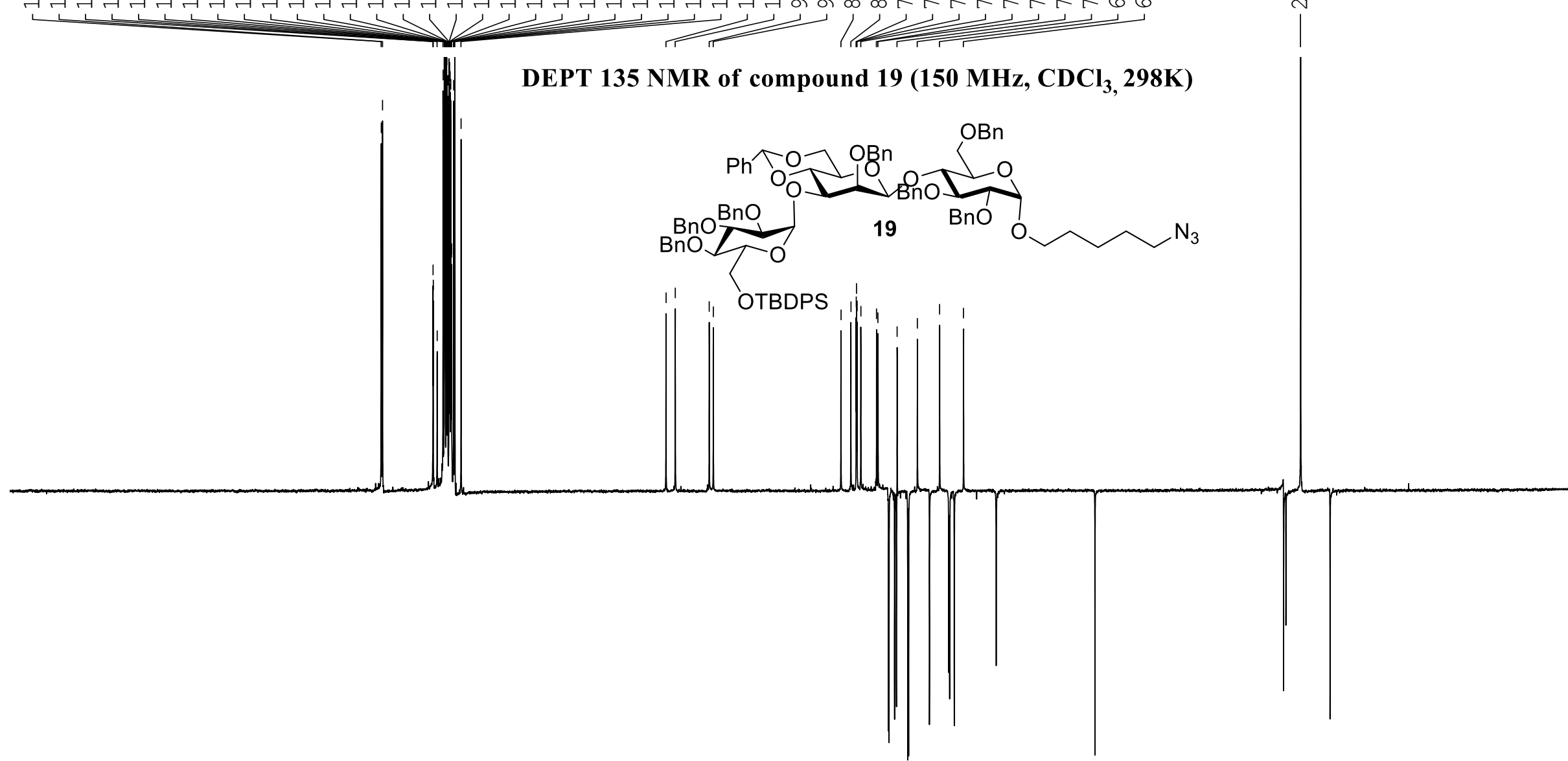




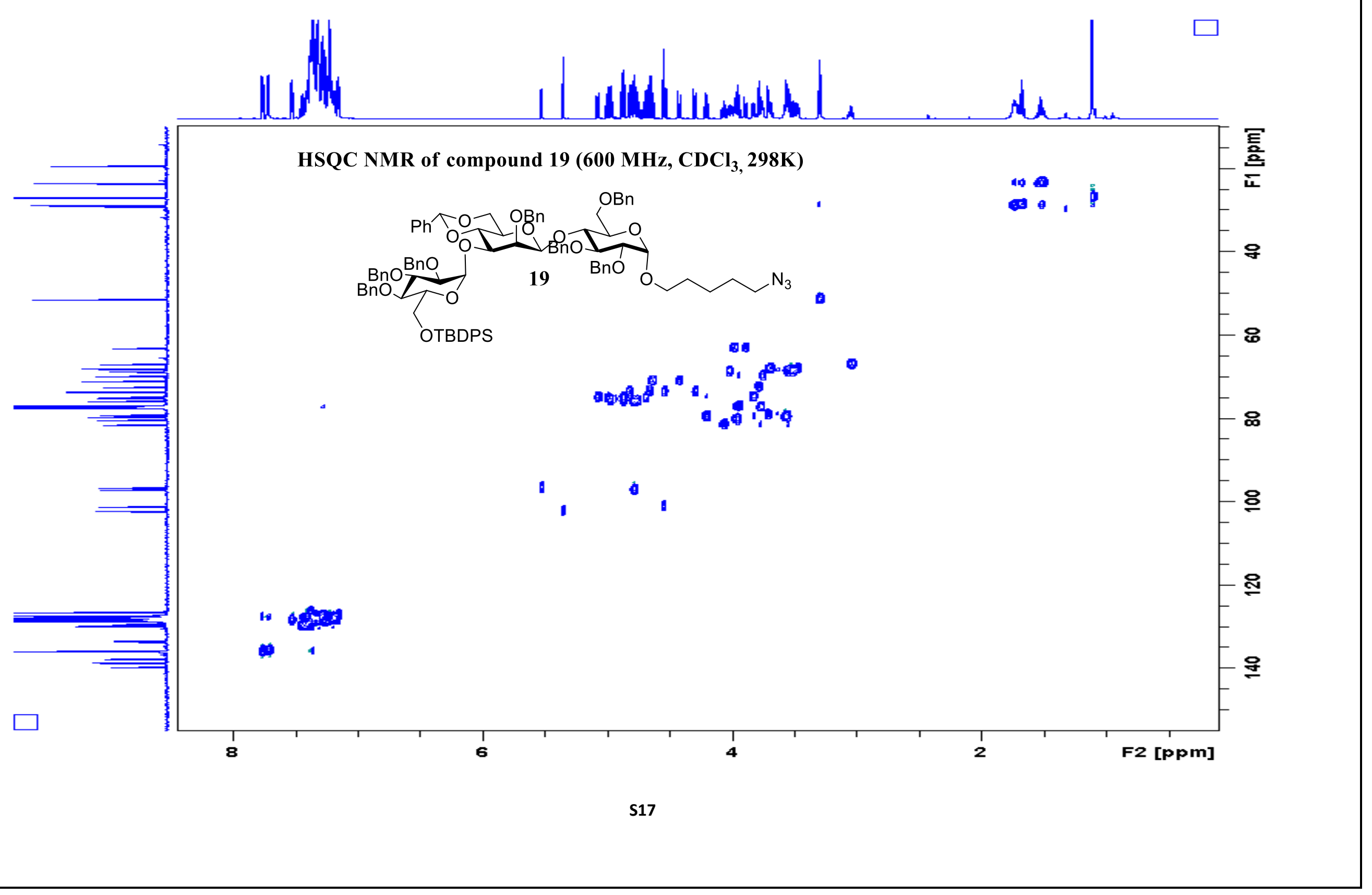




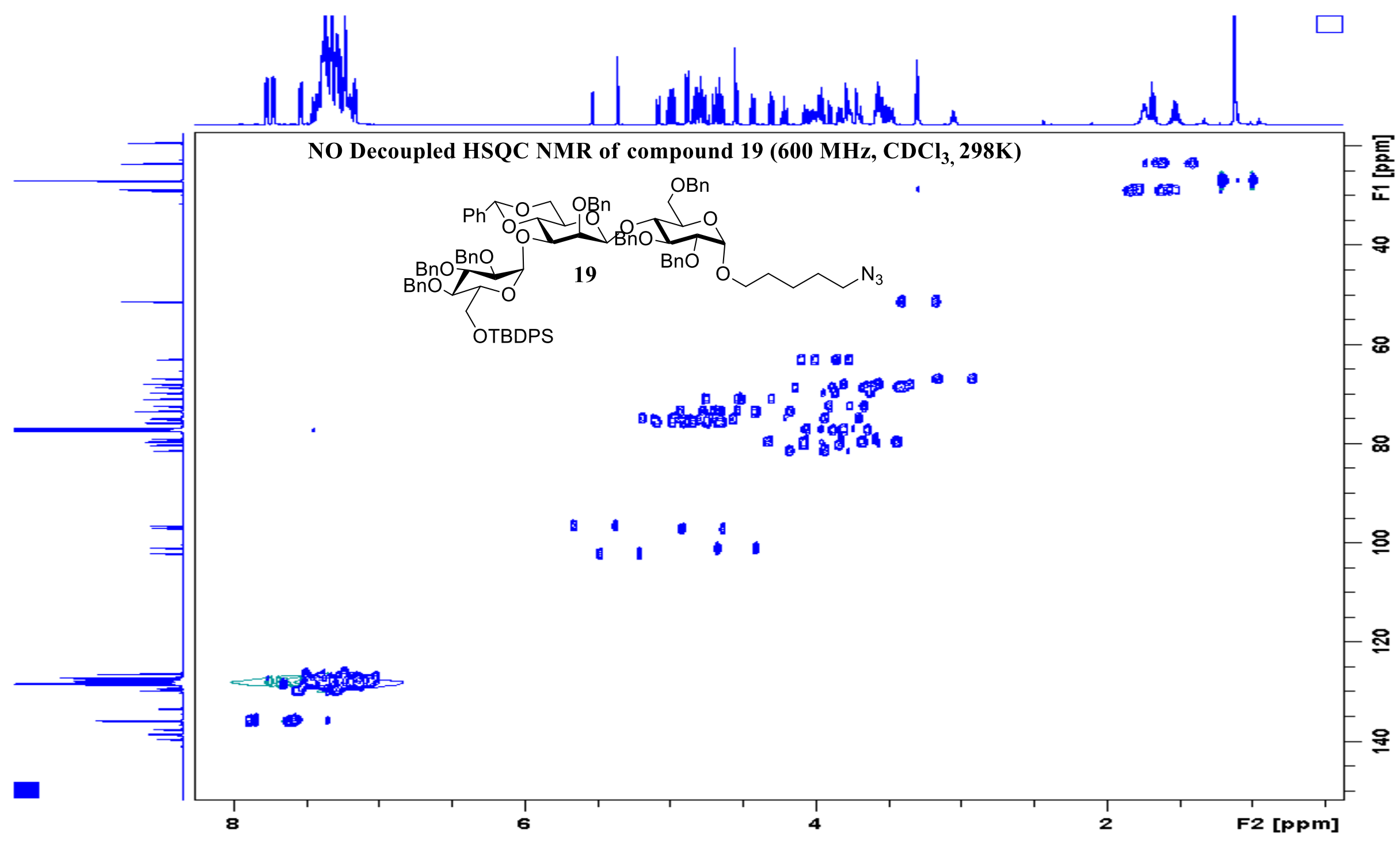

S18 


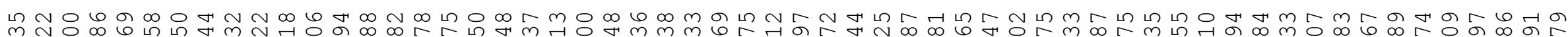

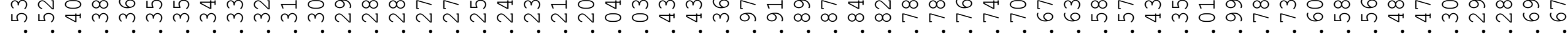

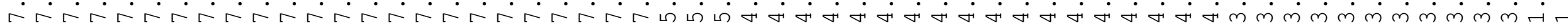

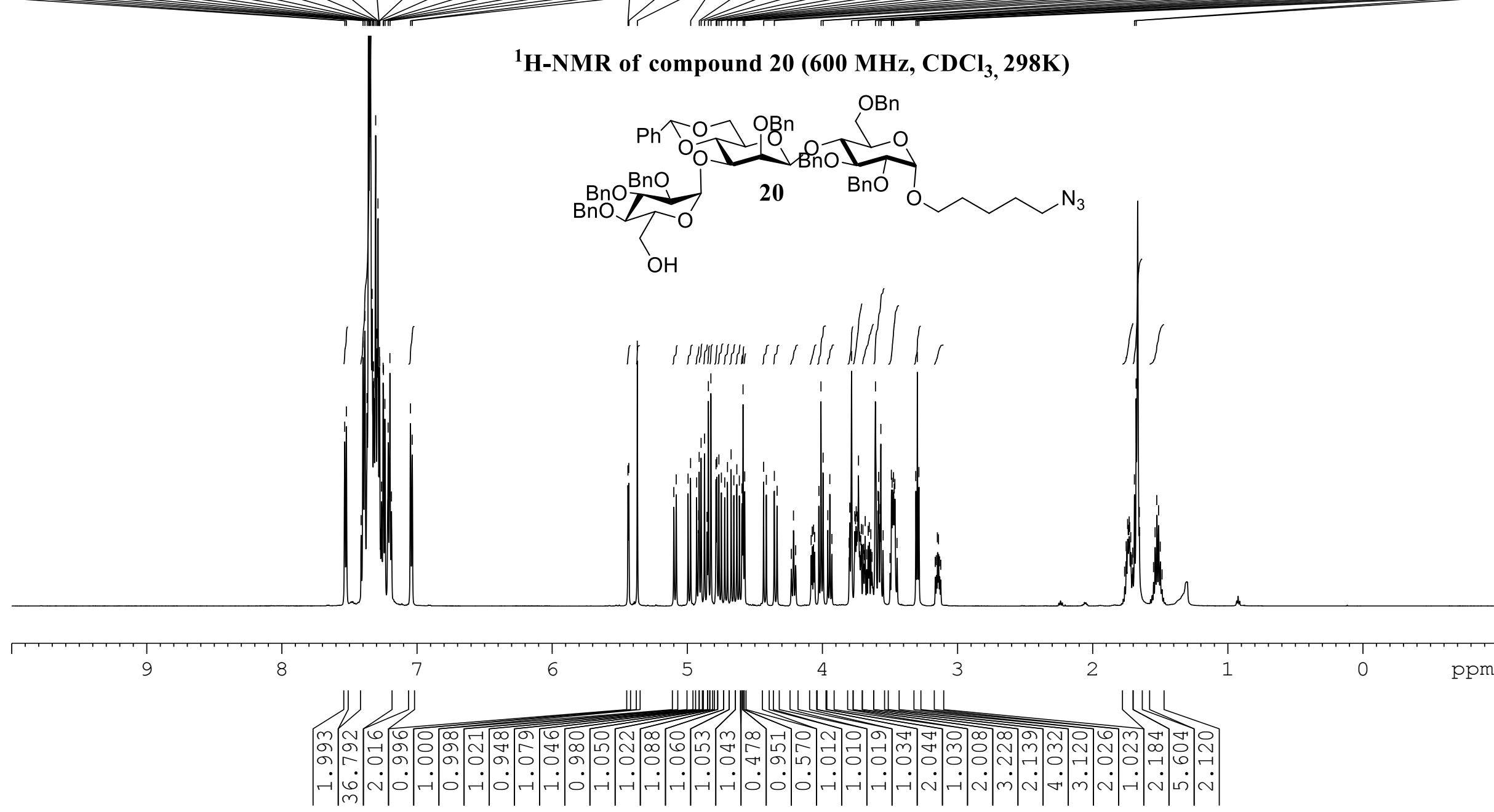




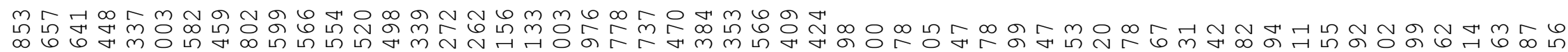

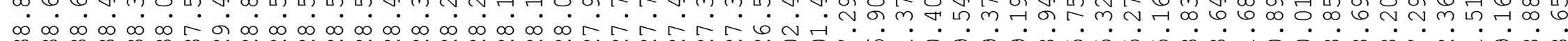

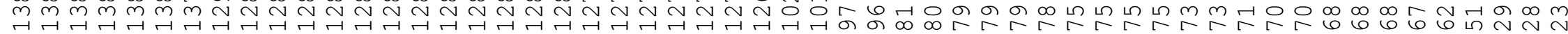

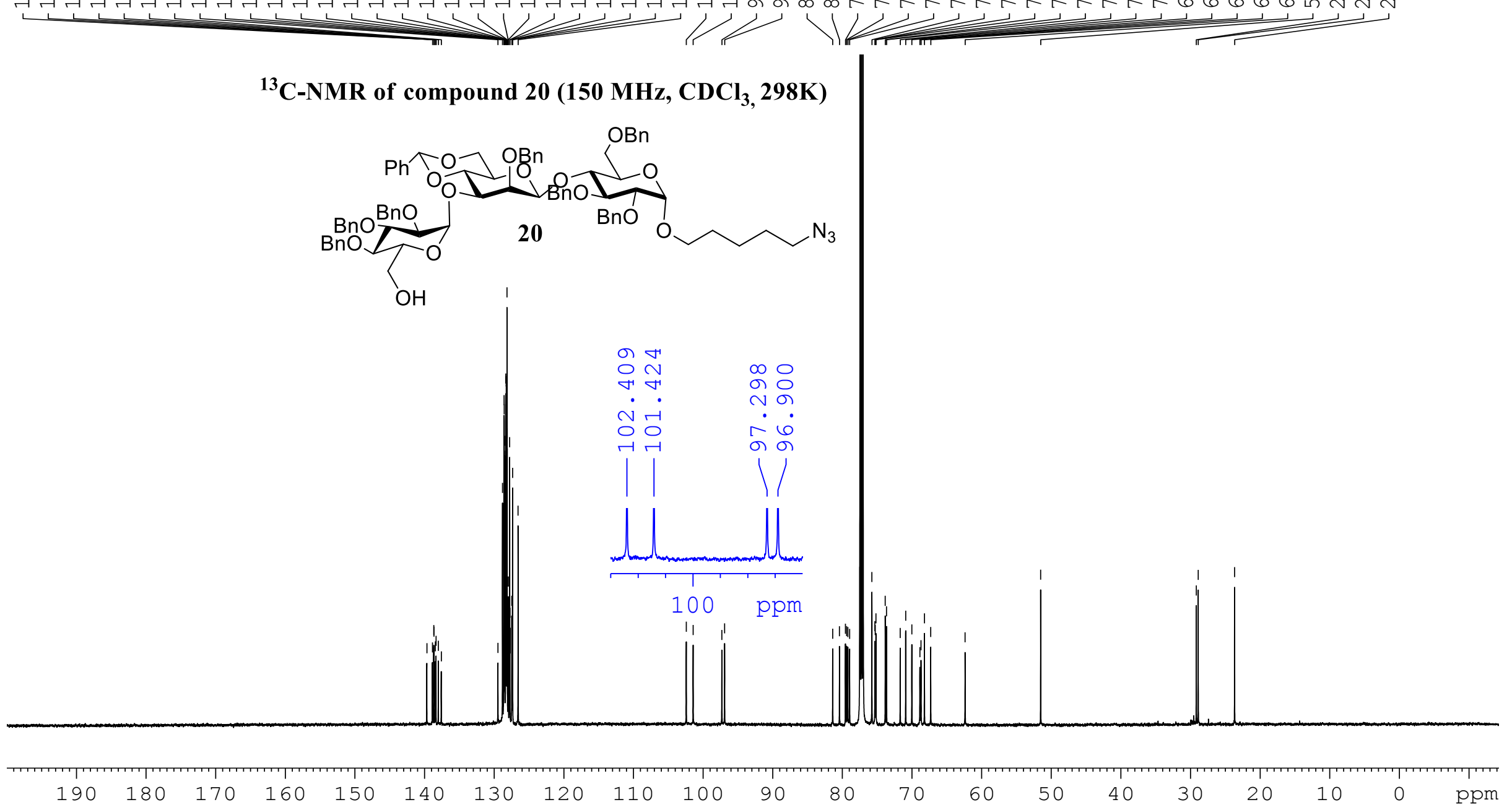




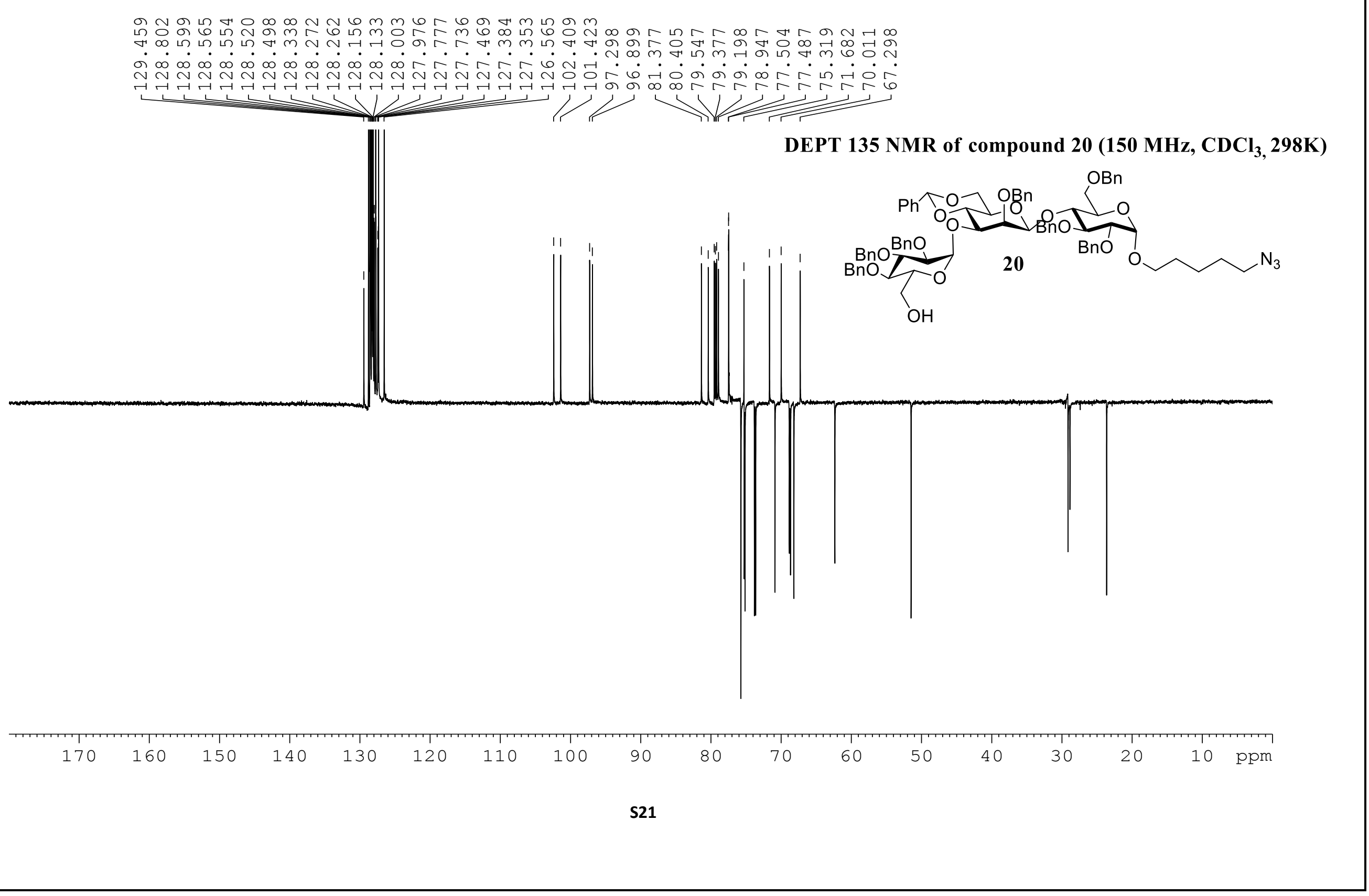




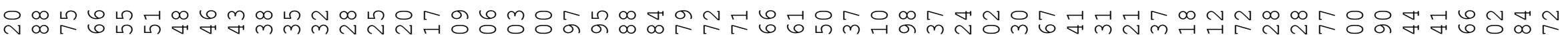

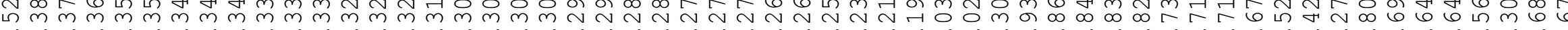

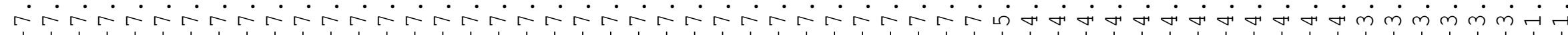

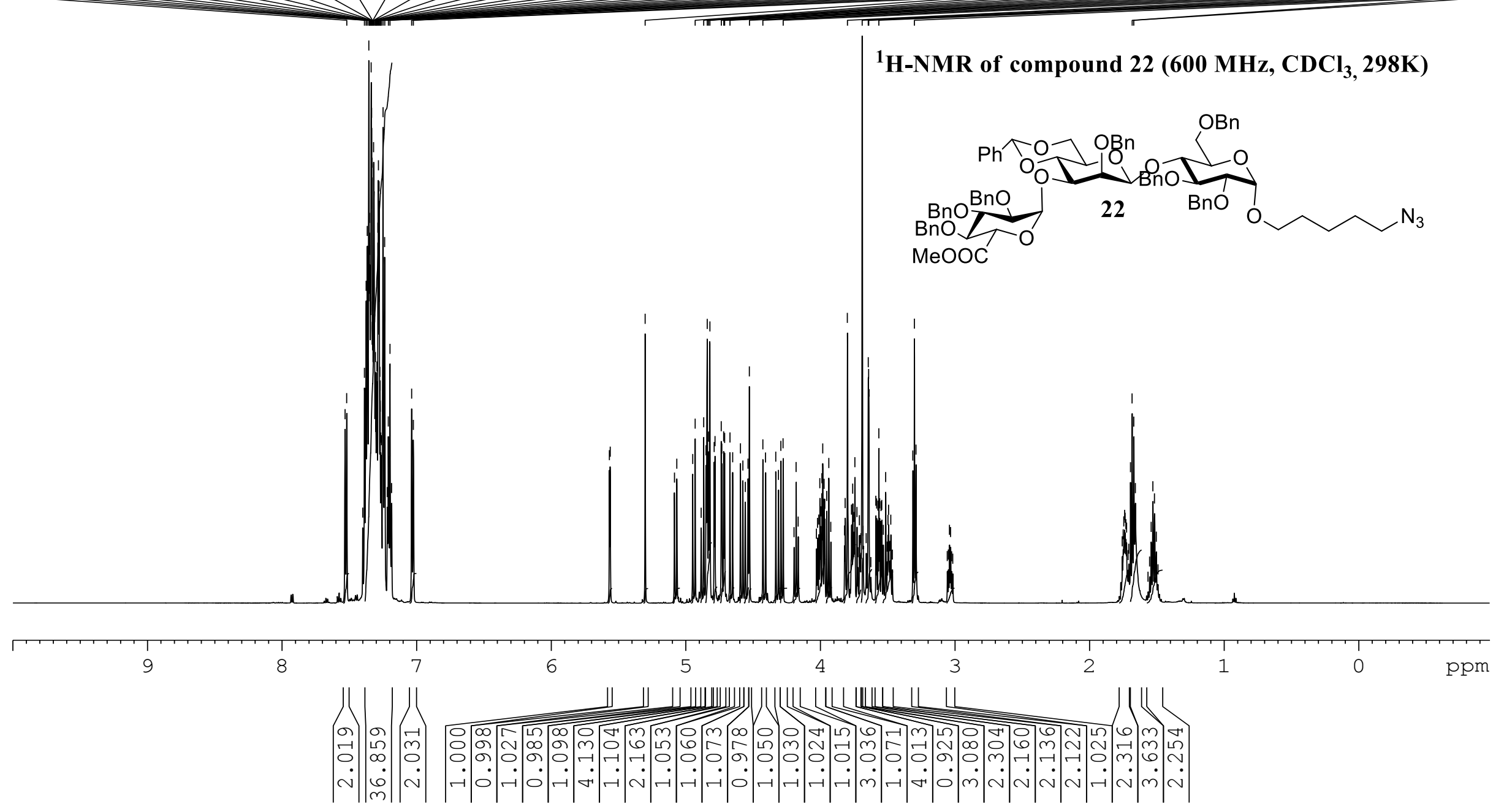


br

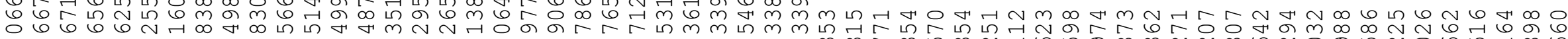

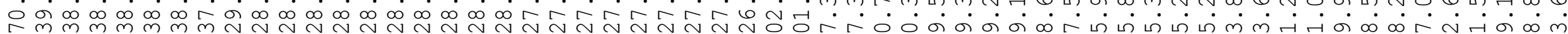

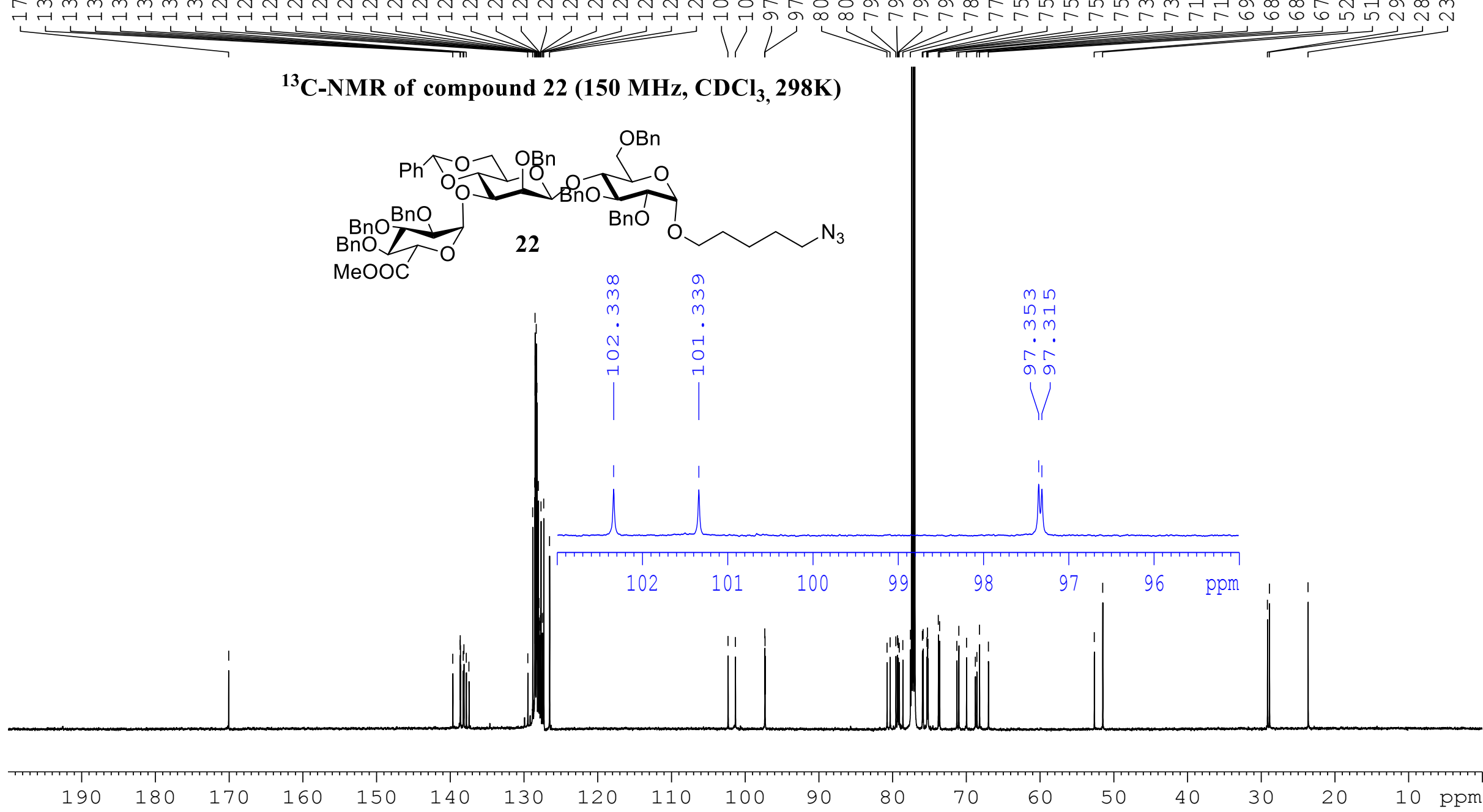




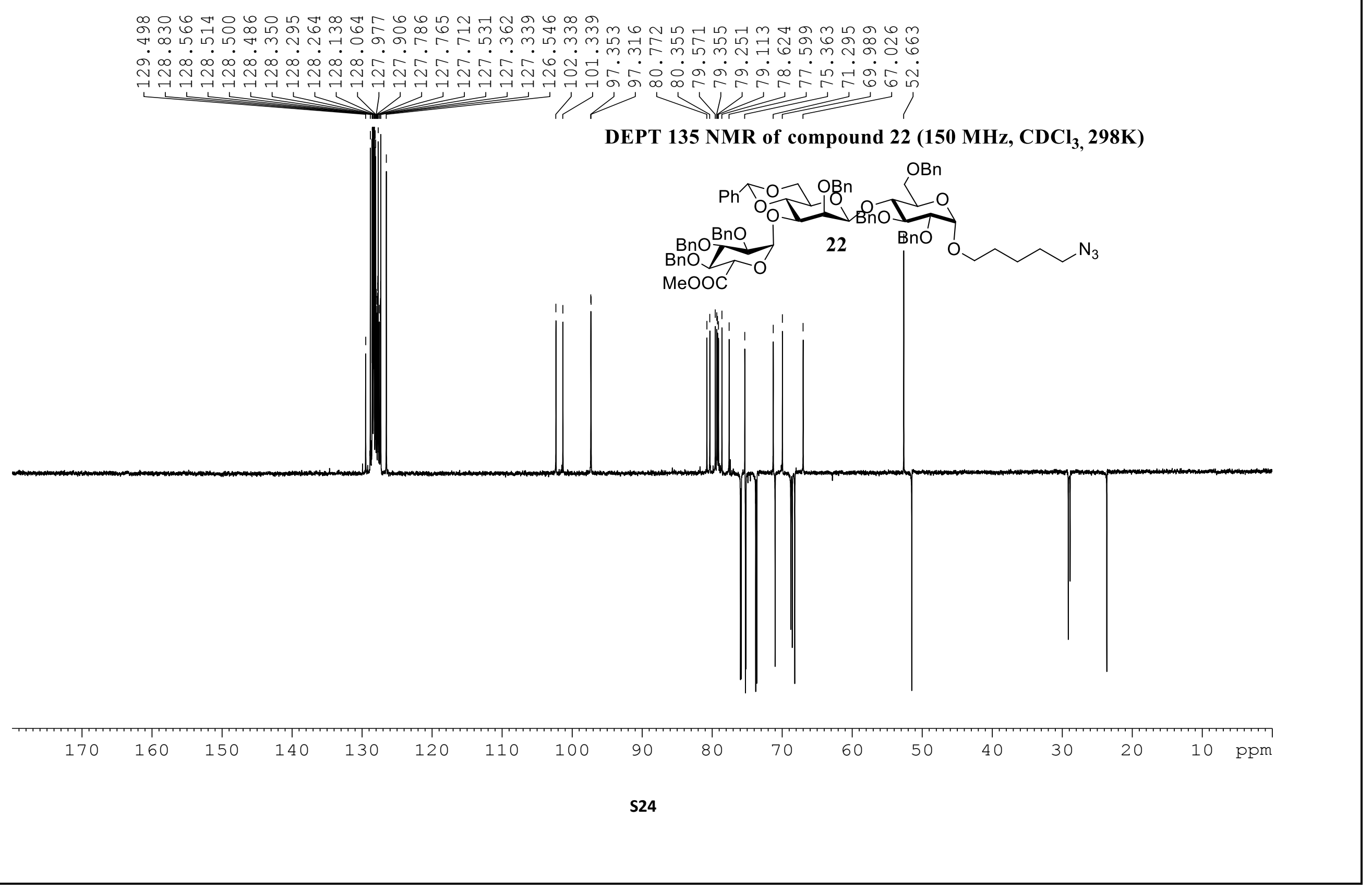




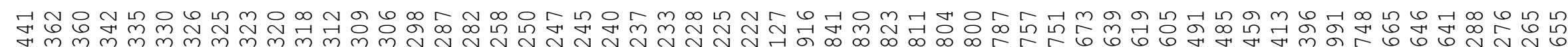

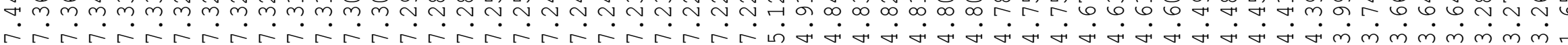

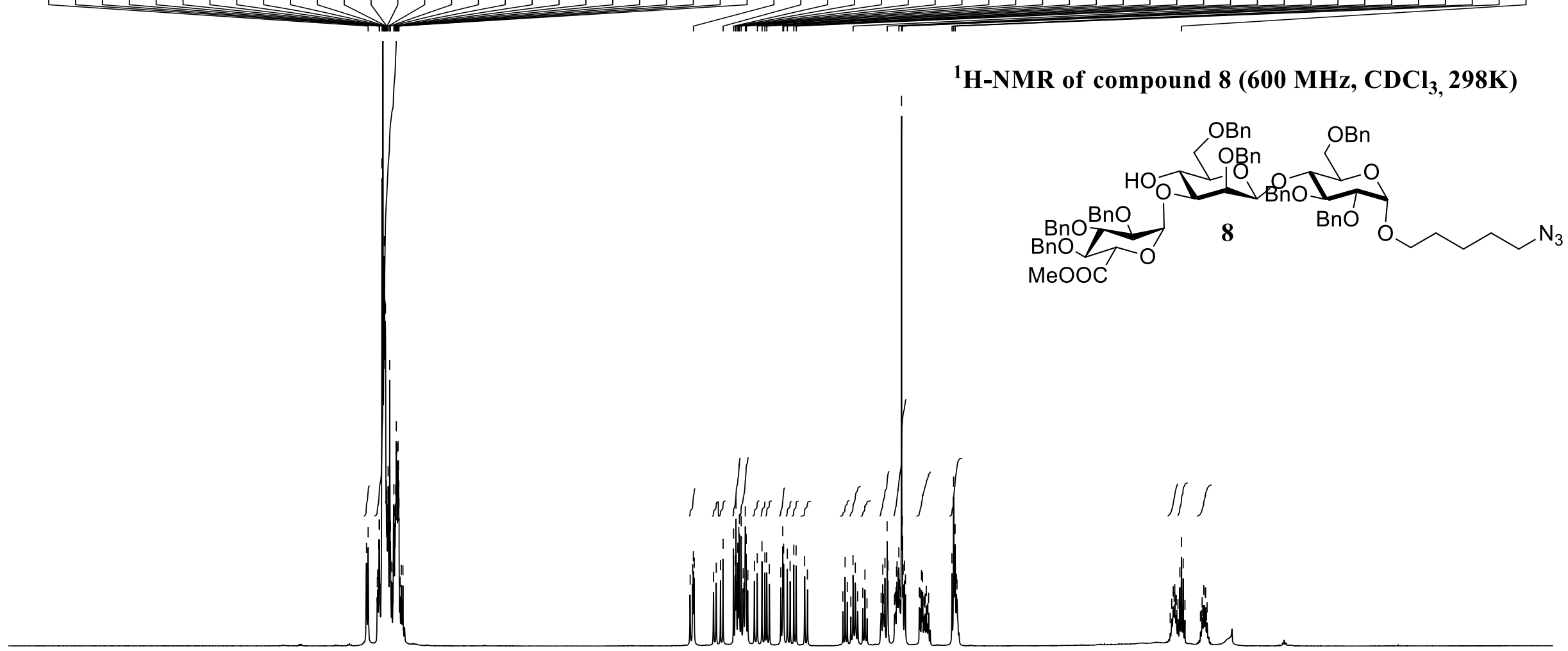

9

8

7

5

4

3
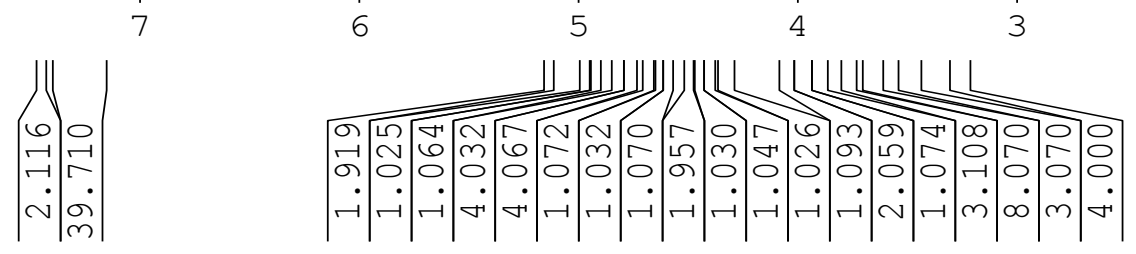

2

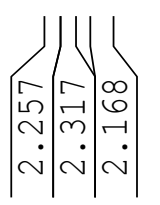

1

ppm

S25 


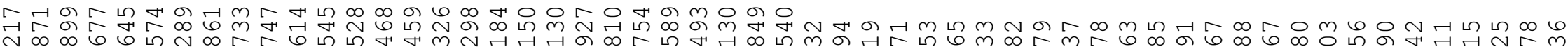

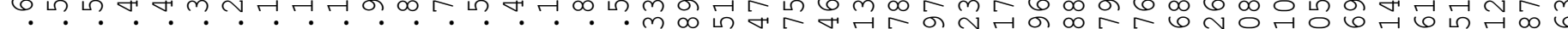

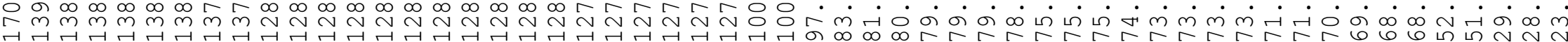

L

${ }^{13} \mathrm{C}-\mathrm{NMR}$ of compound 8 (150 $\left.\mathrm{MHz}, \mathrm{CDCl}_{3}, 298 \mathrm{~K}\right)$
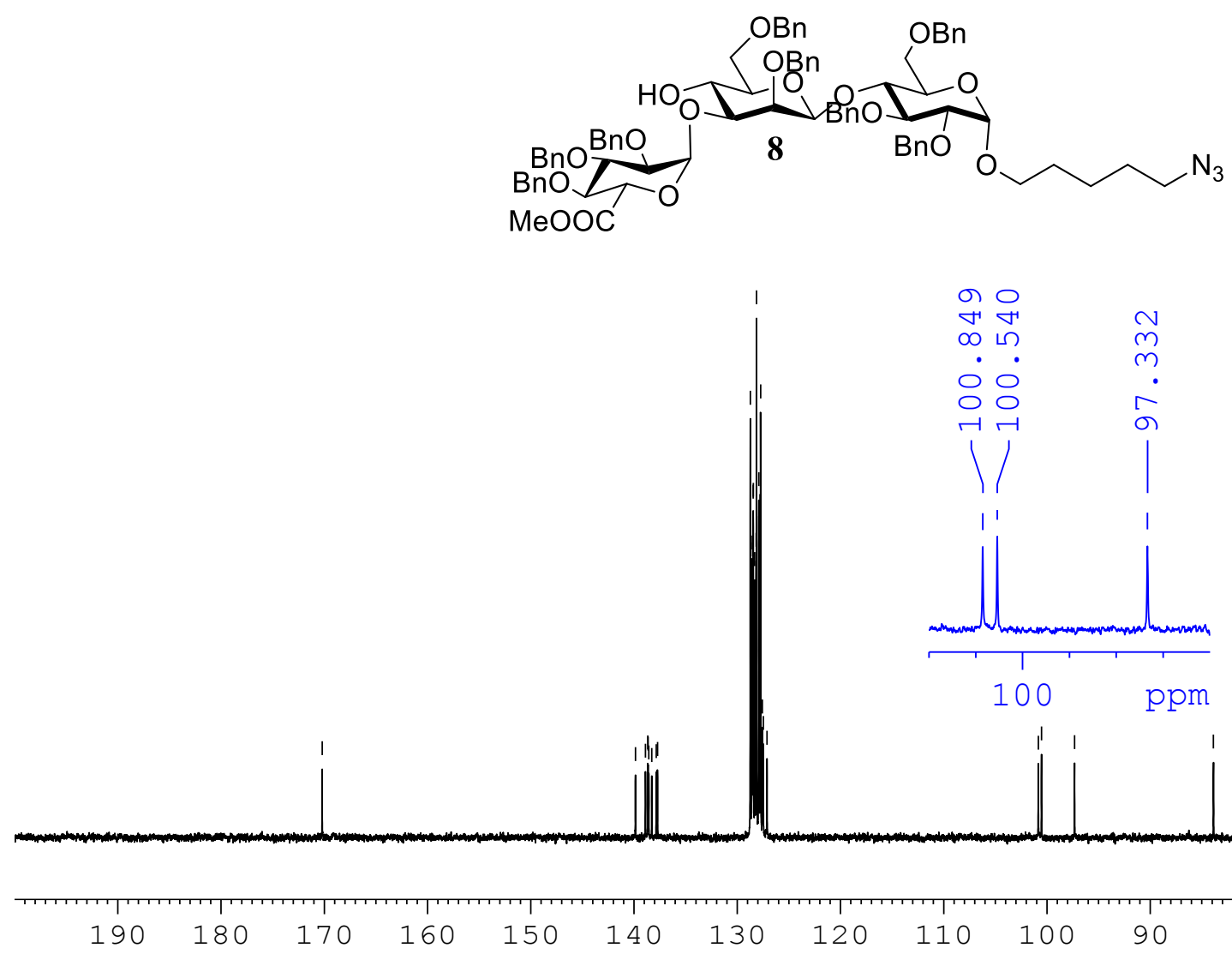

S26 


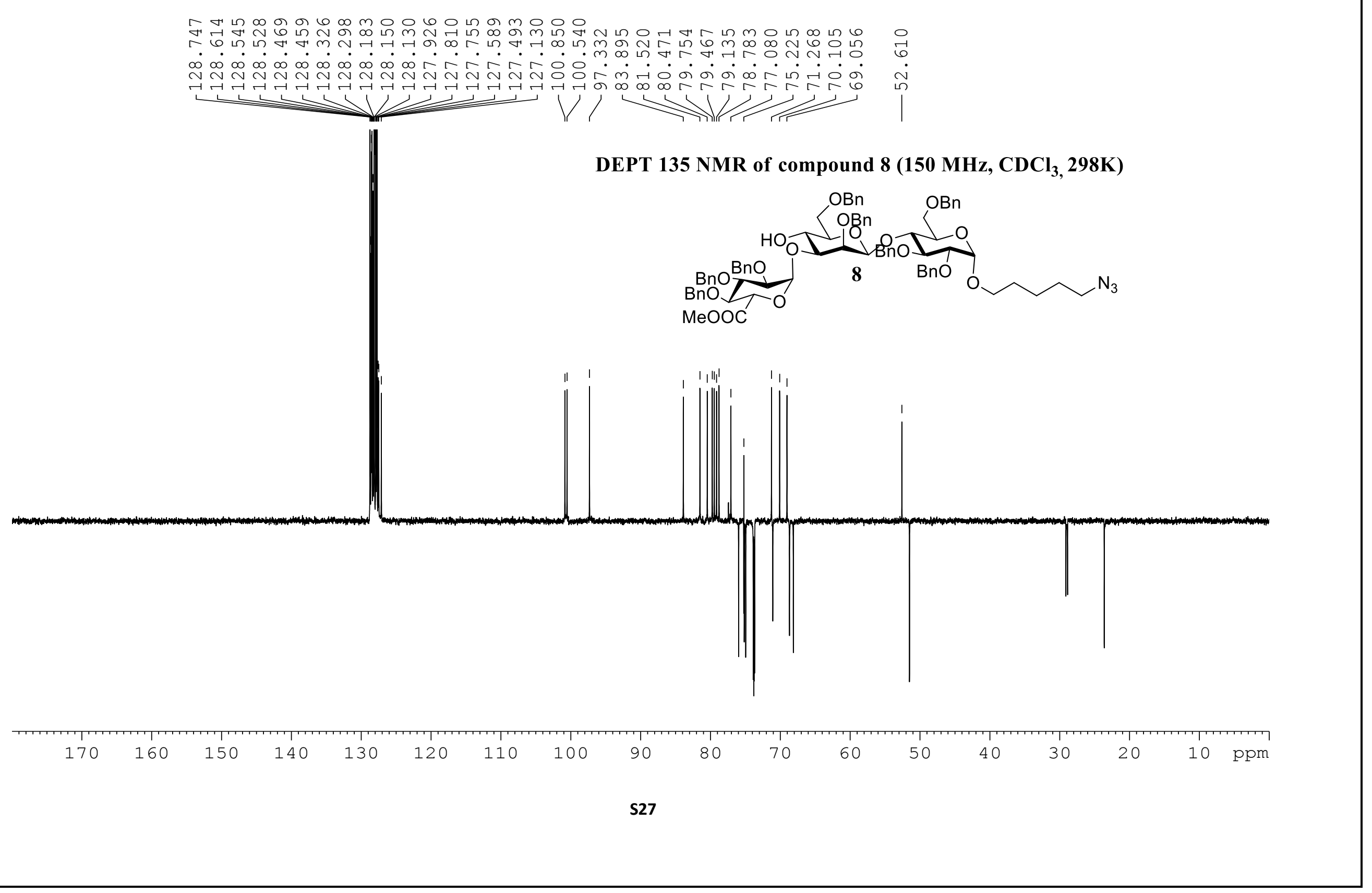




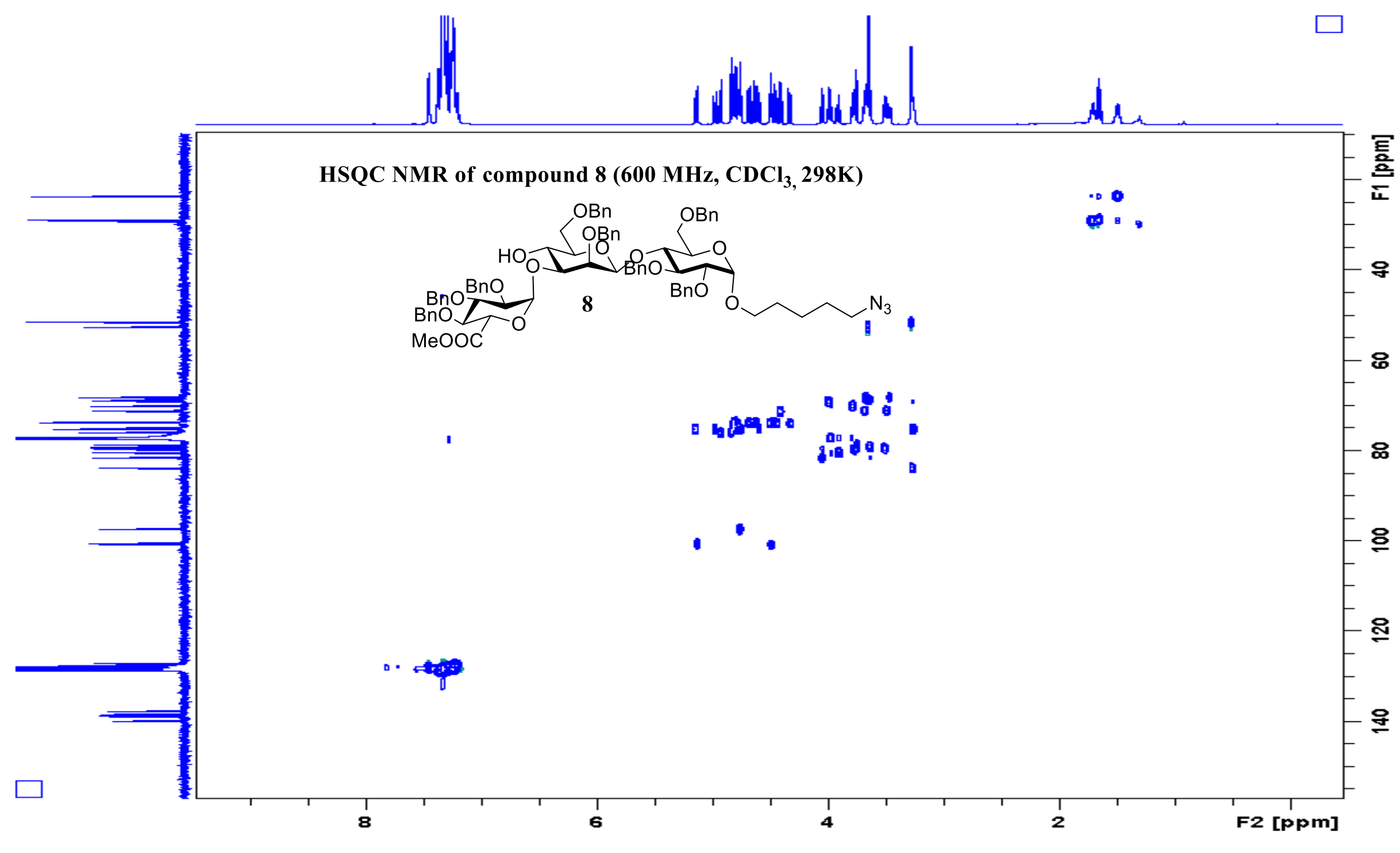




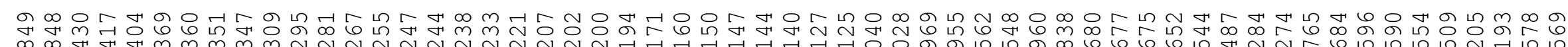

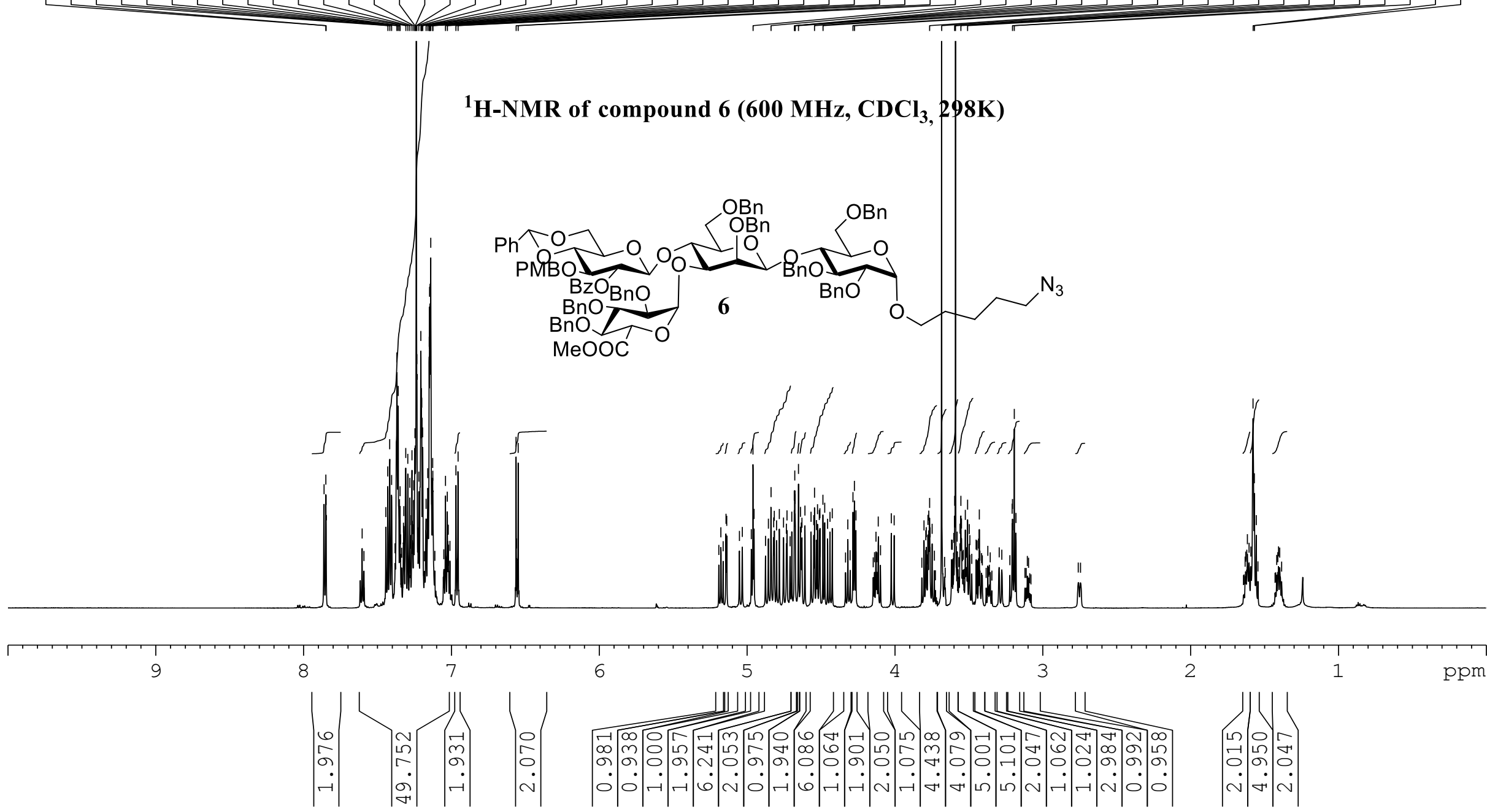




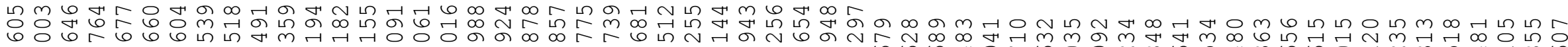

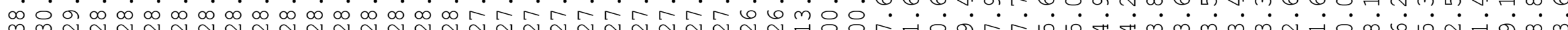

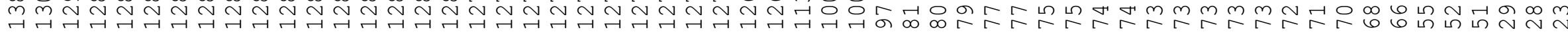

${ }^{13} \mathrm{C}-\mathrm{NMR}$ of compound $6\left(150 \mathrm{MHz}, \mathrm{CDCl}_{3}, 298 \mathrm{~K}\right)$

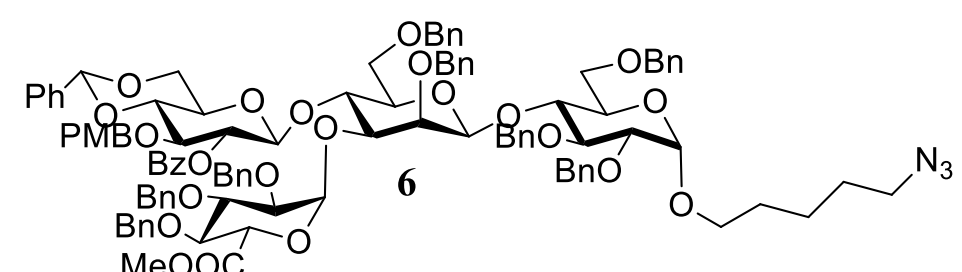

$\mathrm{MeOOC}$

$\curvearrowleft \infty \tilde{r}$

$m$ म

$\dot{0} \dot{0} \dot{0}$

000

다다 다
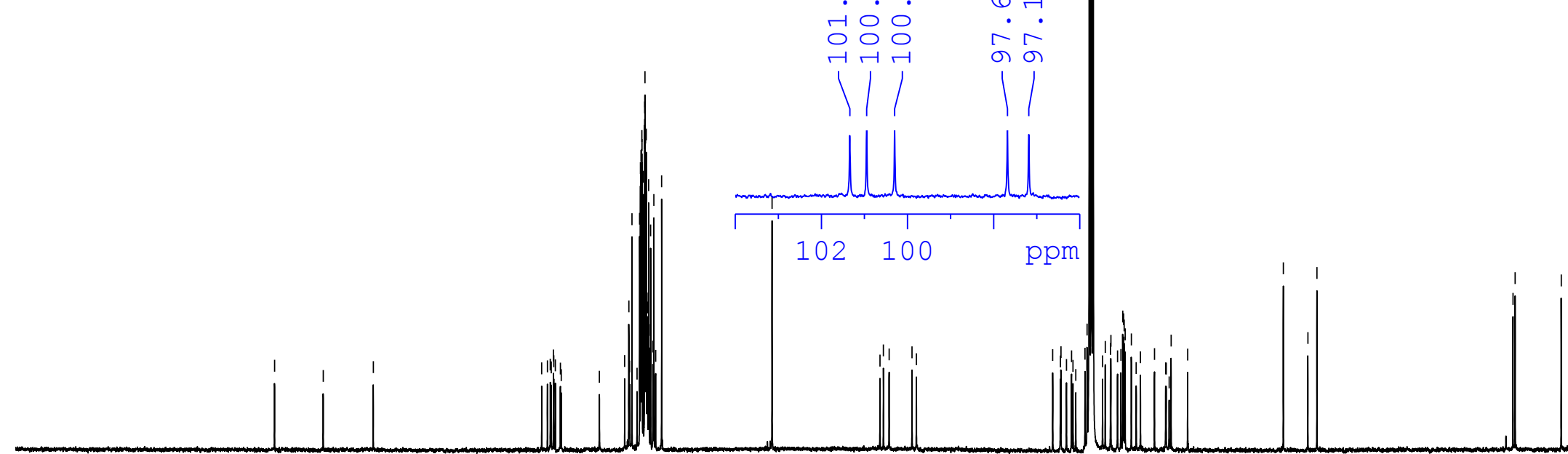

190
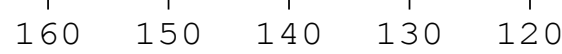

100

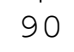

80

60

50

40

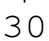

$2010 \mathrm{ppm}$ 


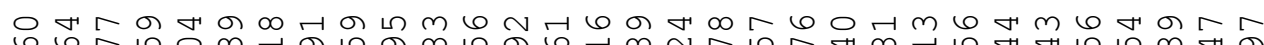

수의

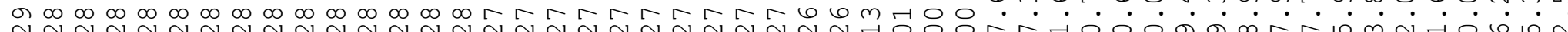

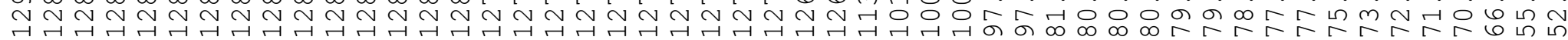

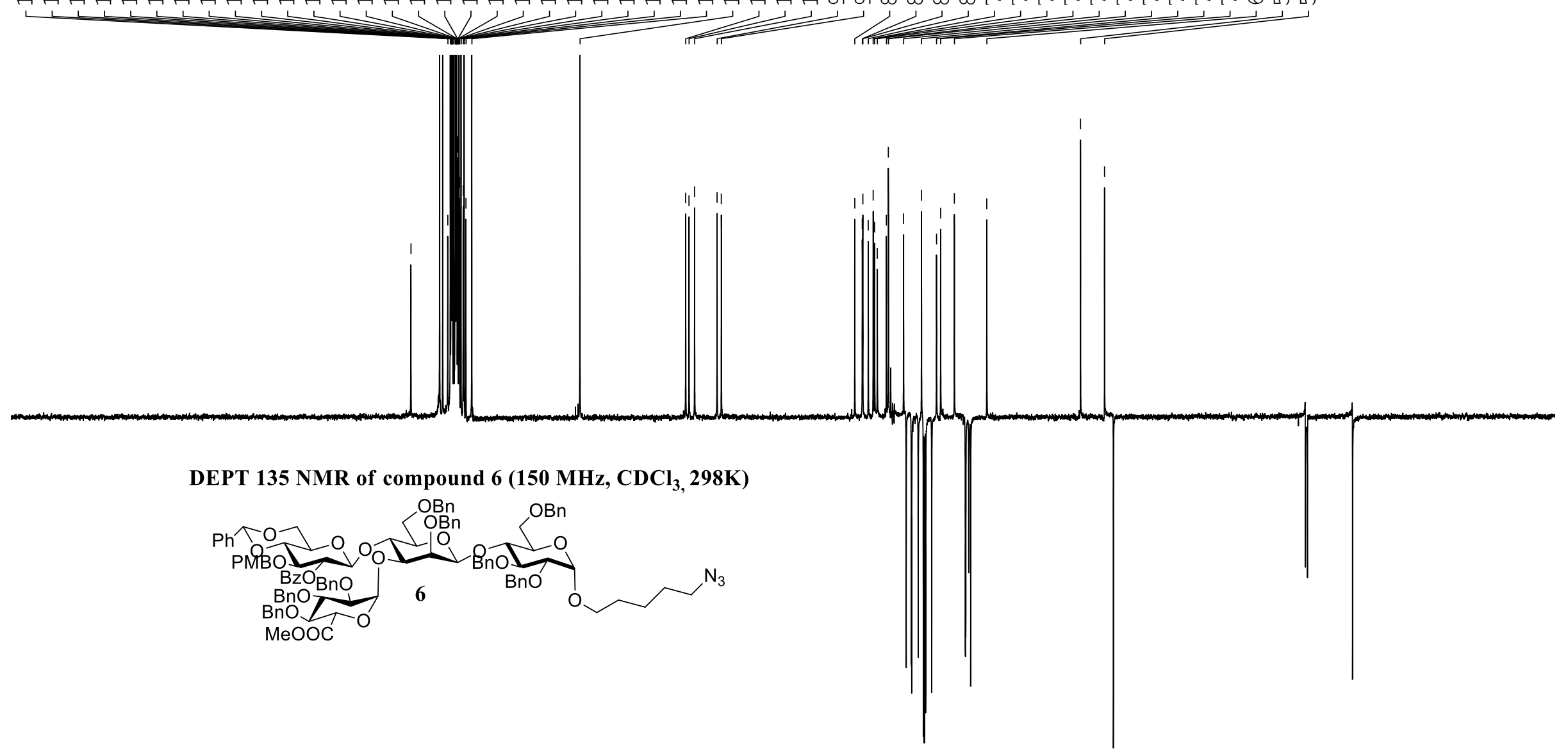




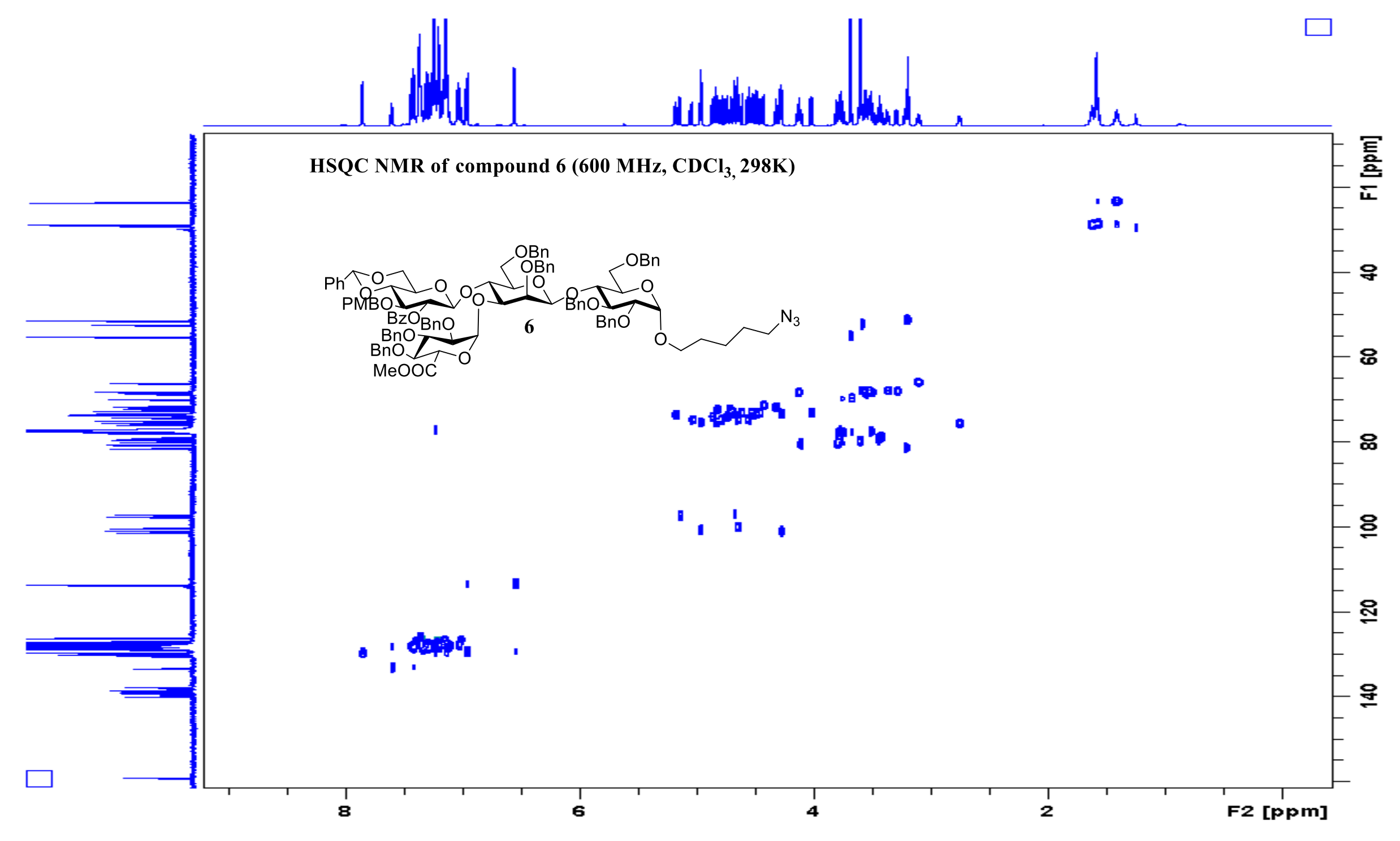




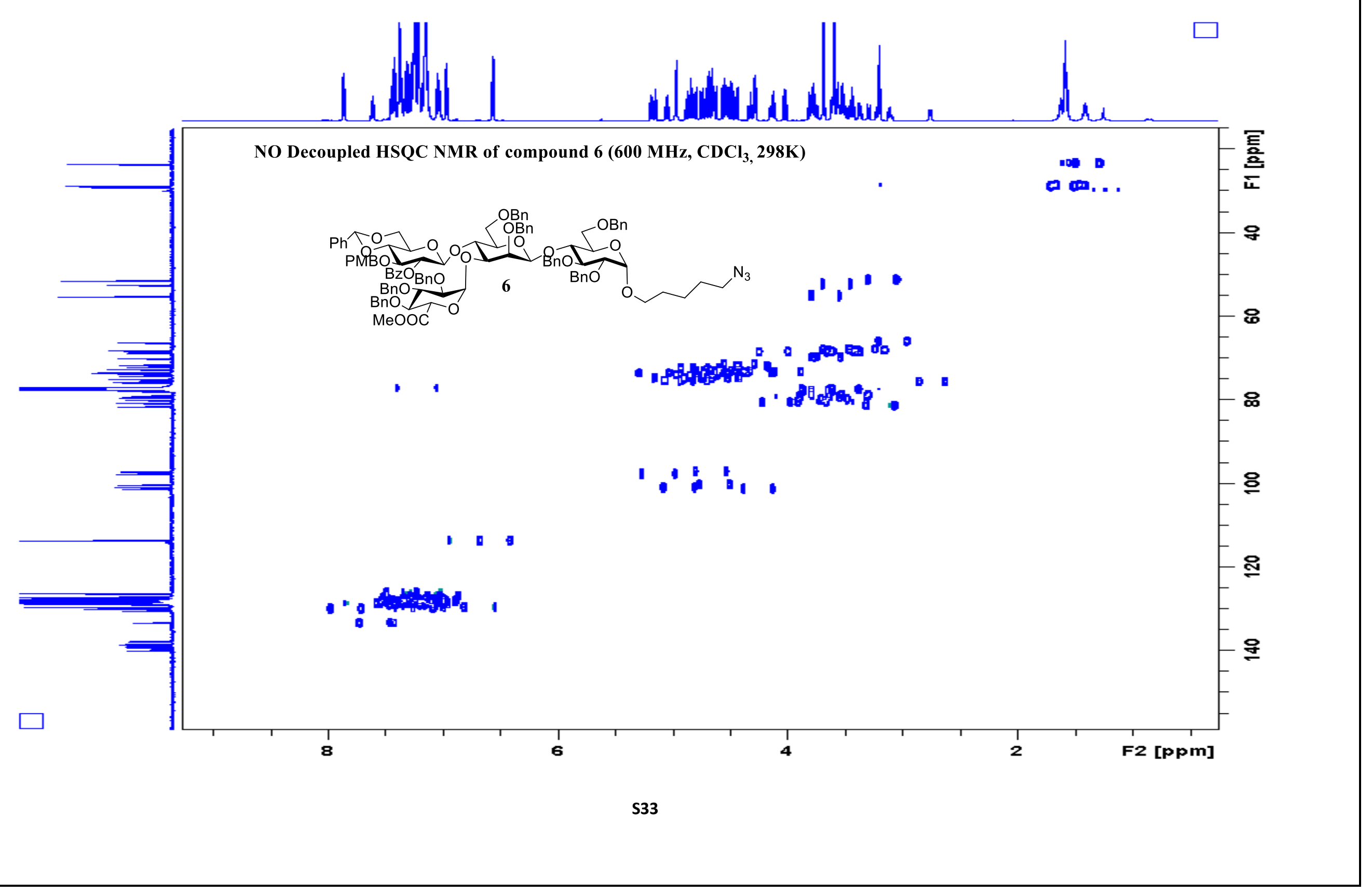




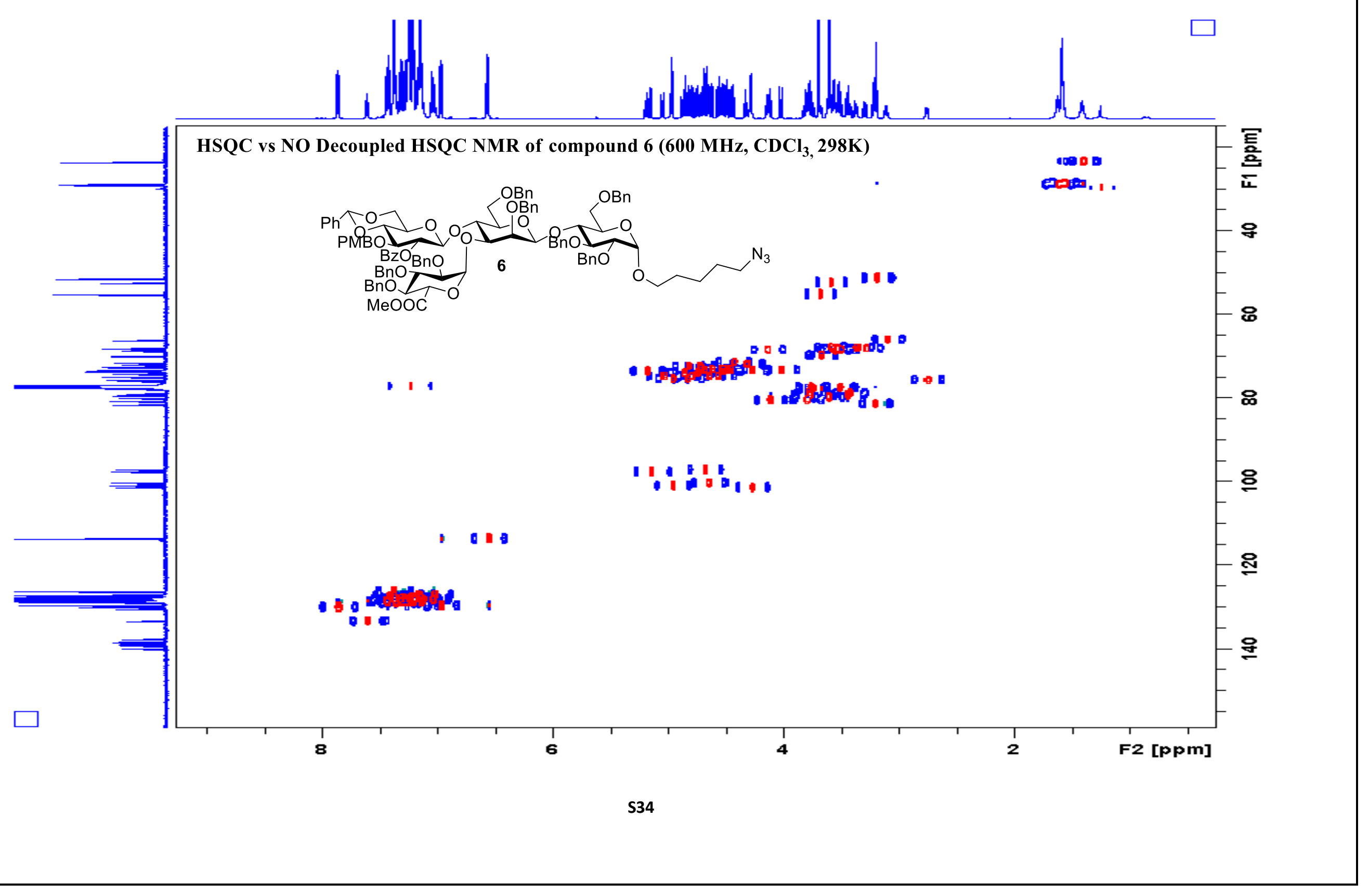




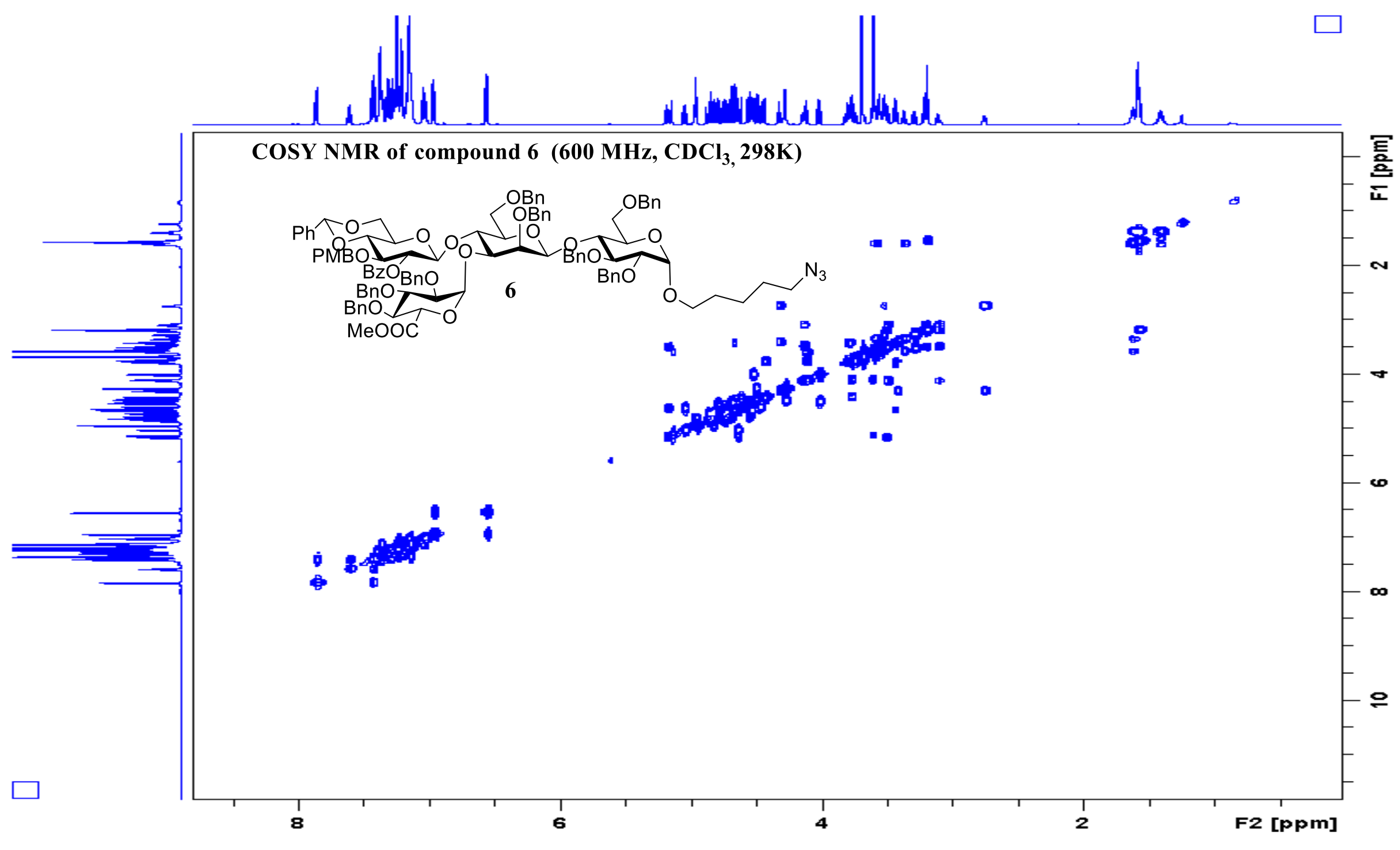




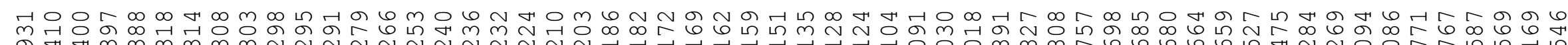
mmmmN N N N N N N N N

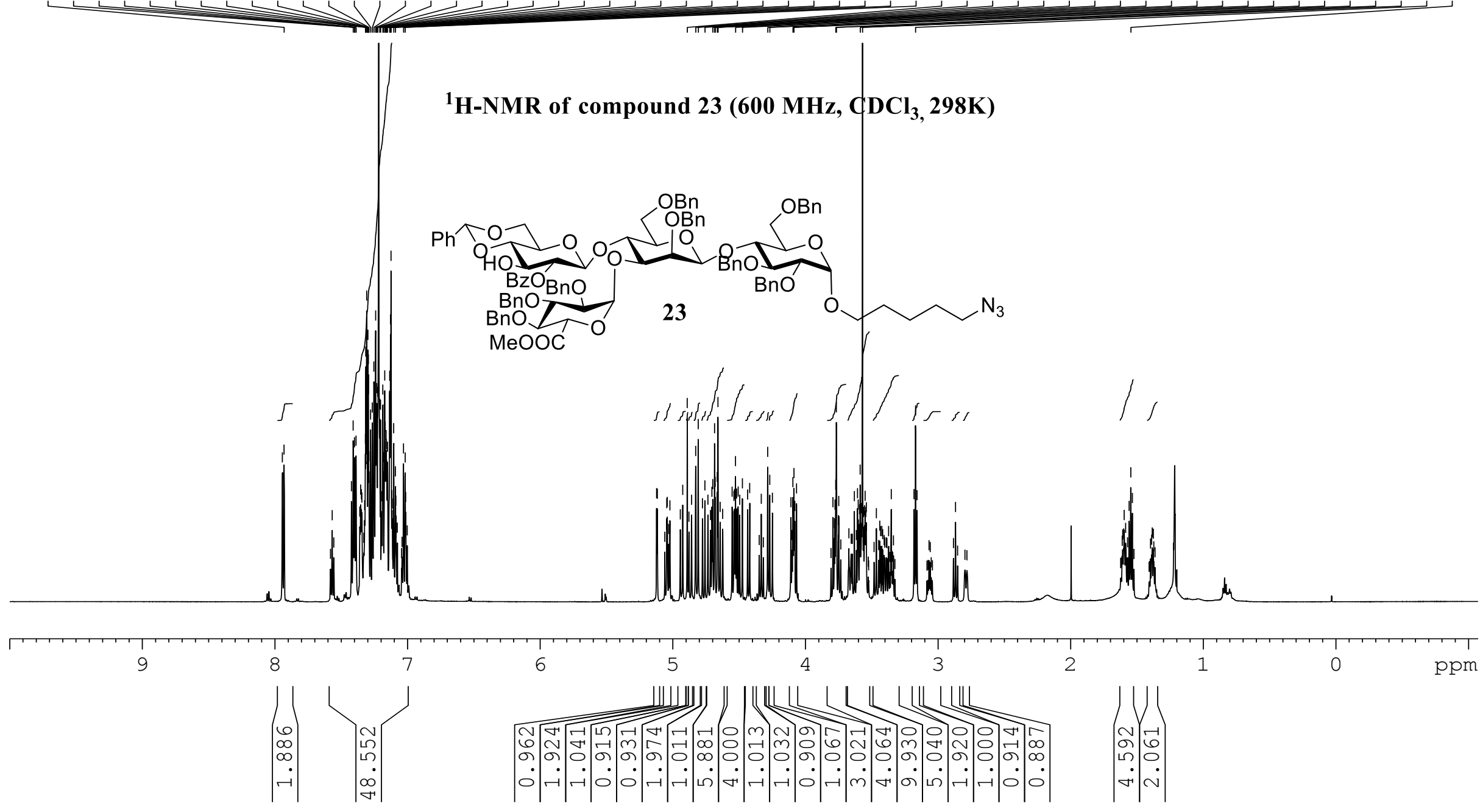




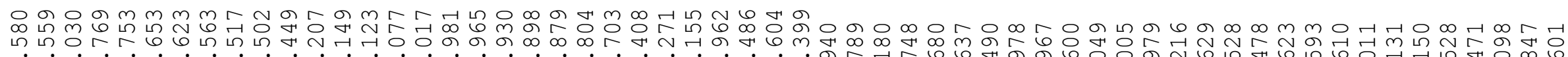

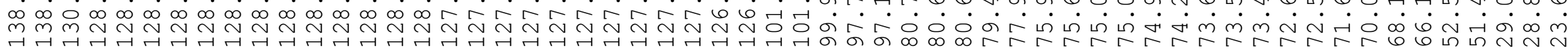

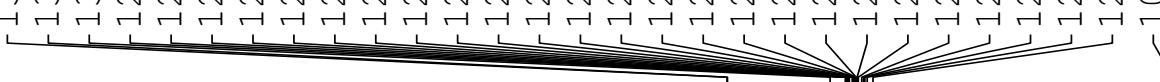

${ }^{13} \mathrm{C}$-NMR of compound 23 (150 $\left.\mathrm{MHz}, \mathrm{CDCl}_{3}, 298 \mathrm{~K}\right)$

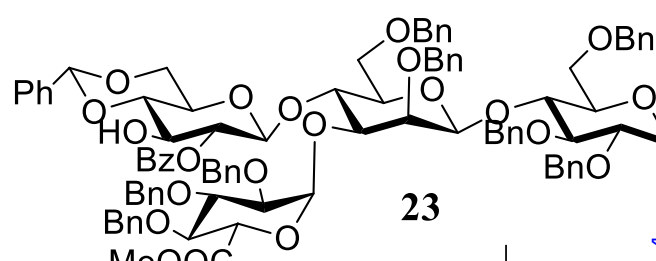

MeOOC

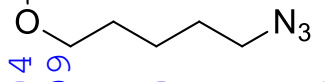

$\infty$

$r$

l.m.

तु

तन

$\therefore r$

1
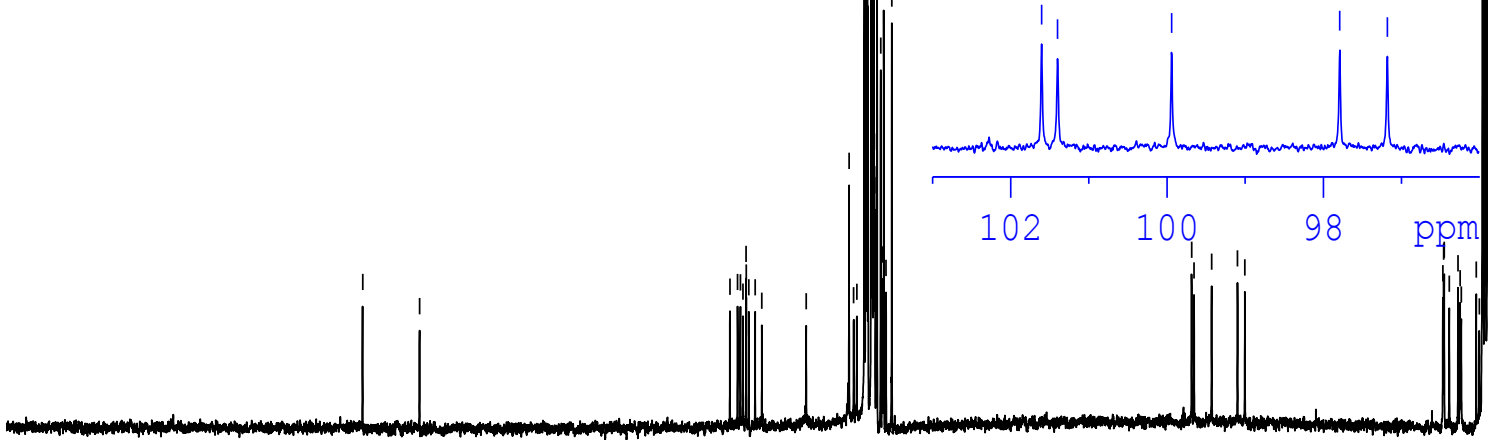

10

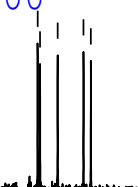

190 


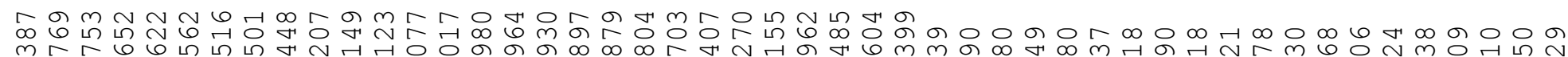

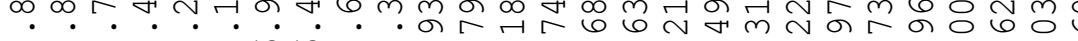

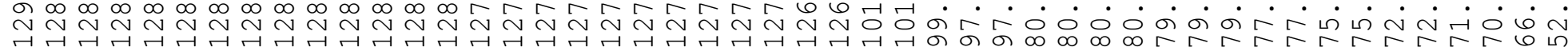

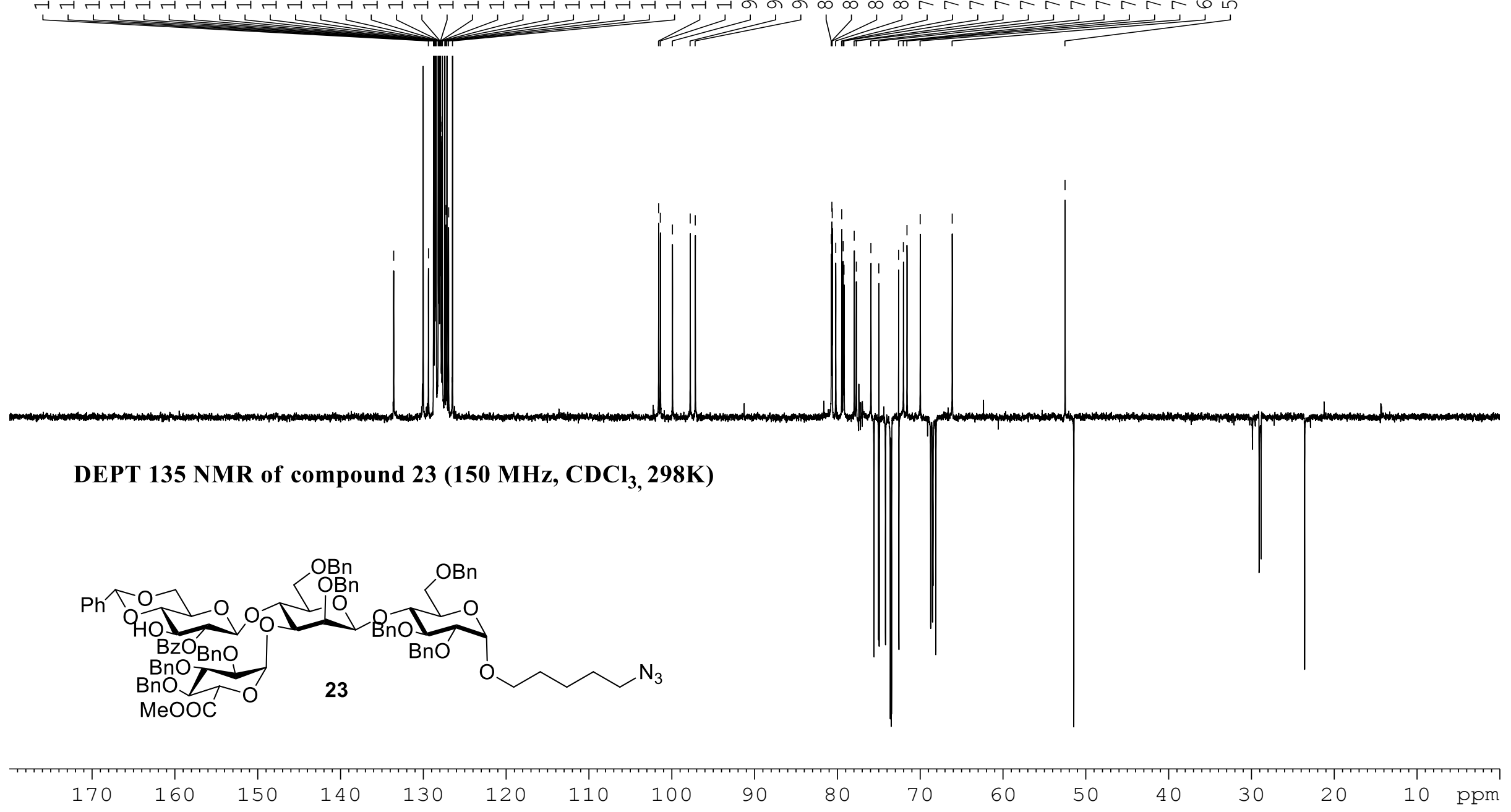




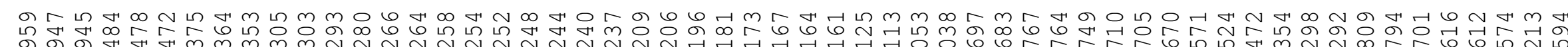

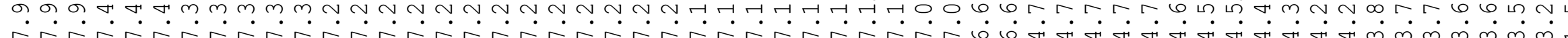

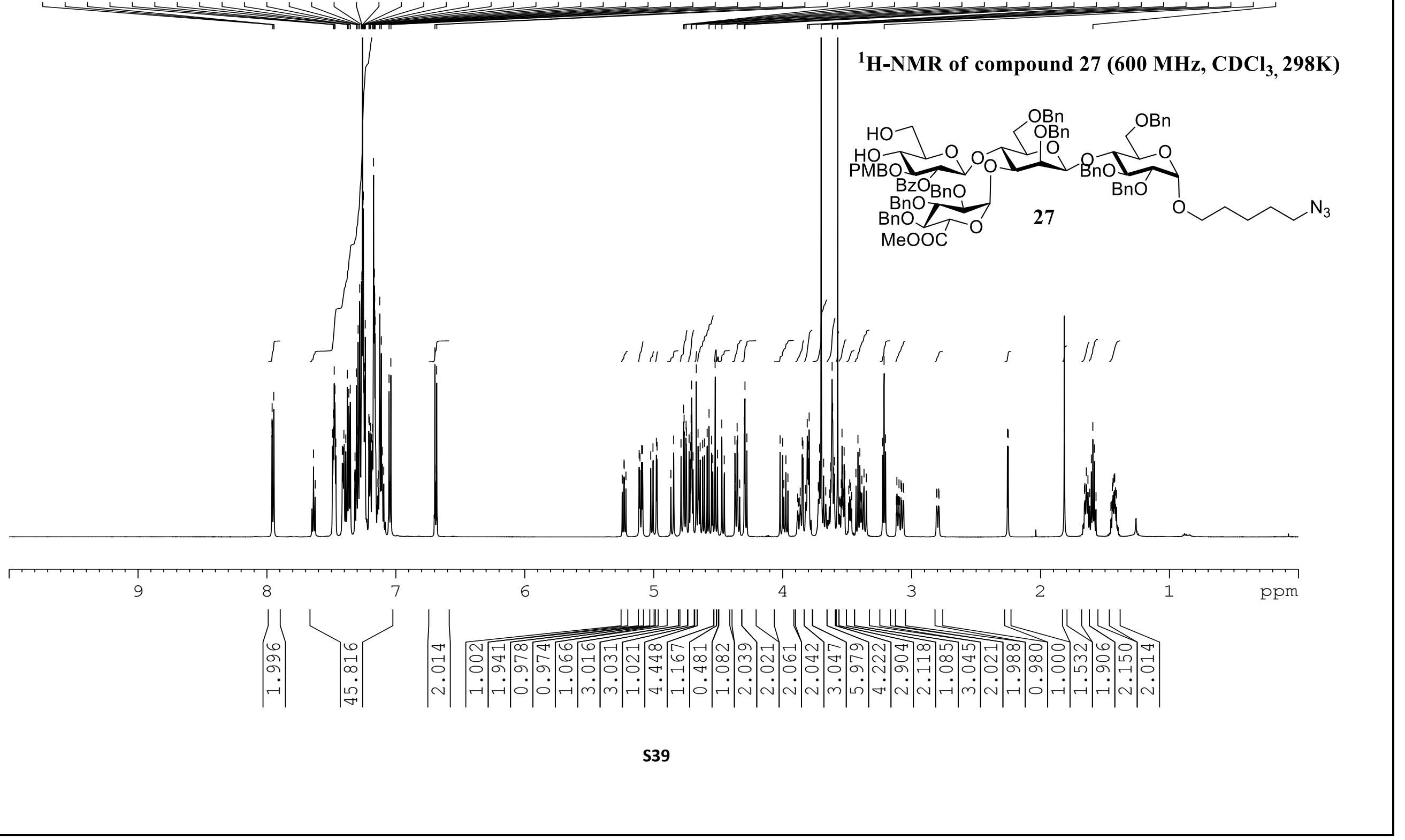




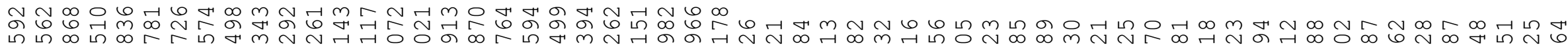

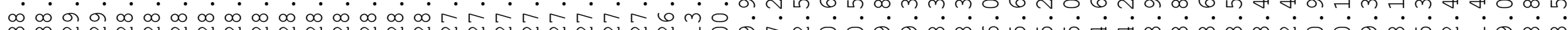

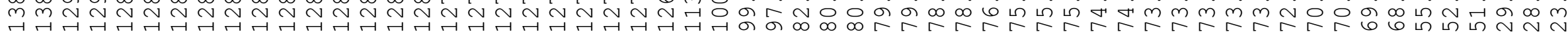

\section{${ }^{13} \mathrm{C}-\mathrm{NMR}$ of compound 27 (150 $\left.\mathrm{MHz}, \mathrm{CDCl}_{3}, 298 \mathrm{~K}\right)$}

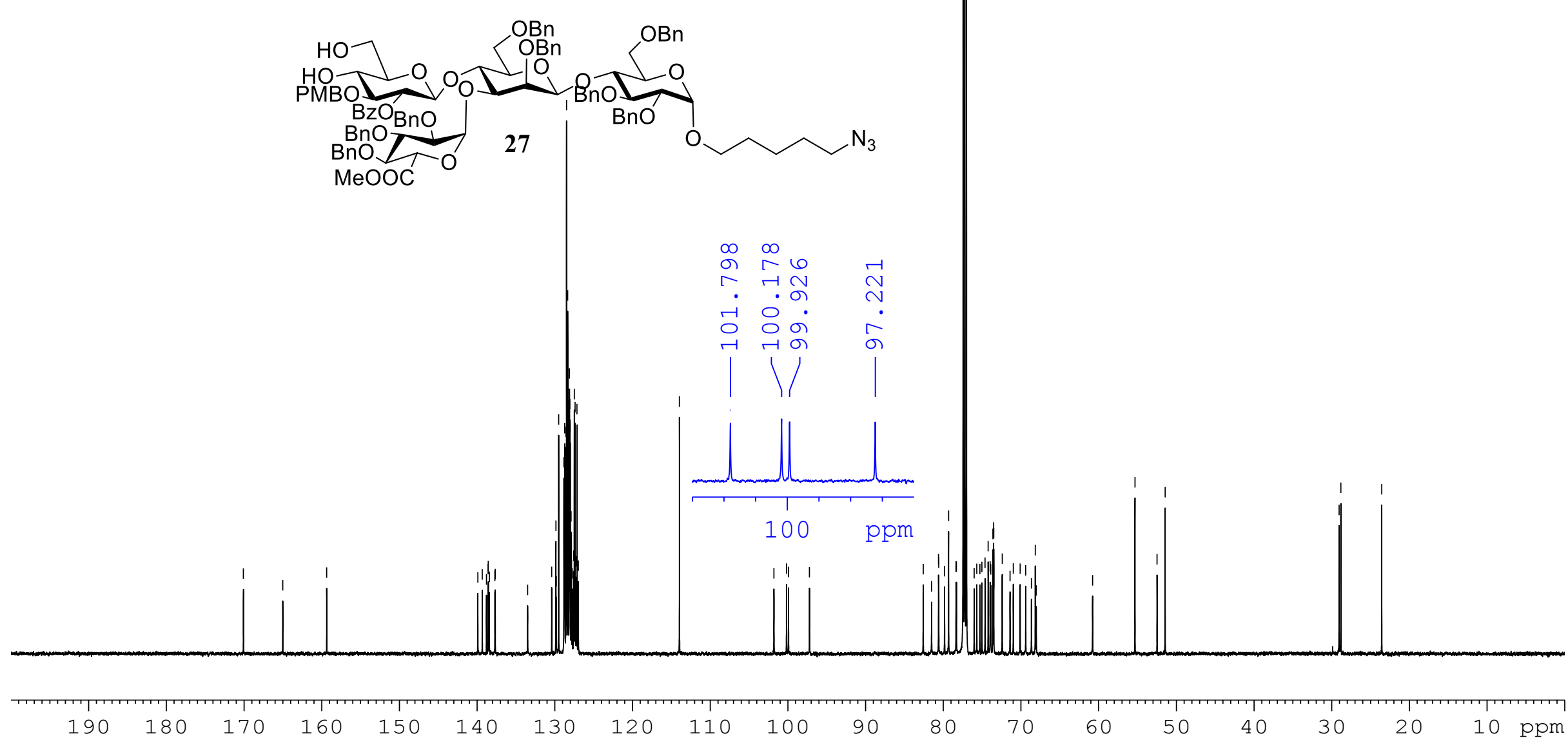




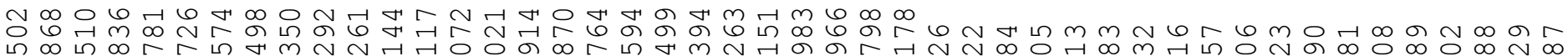
等

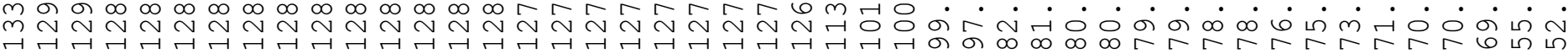

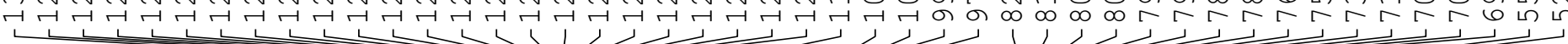

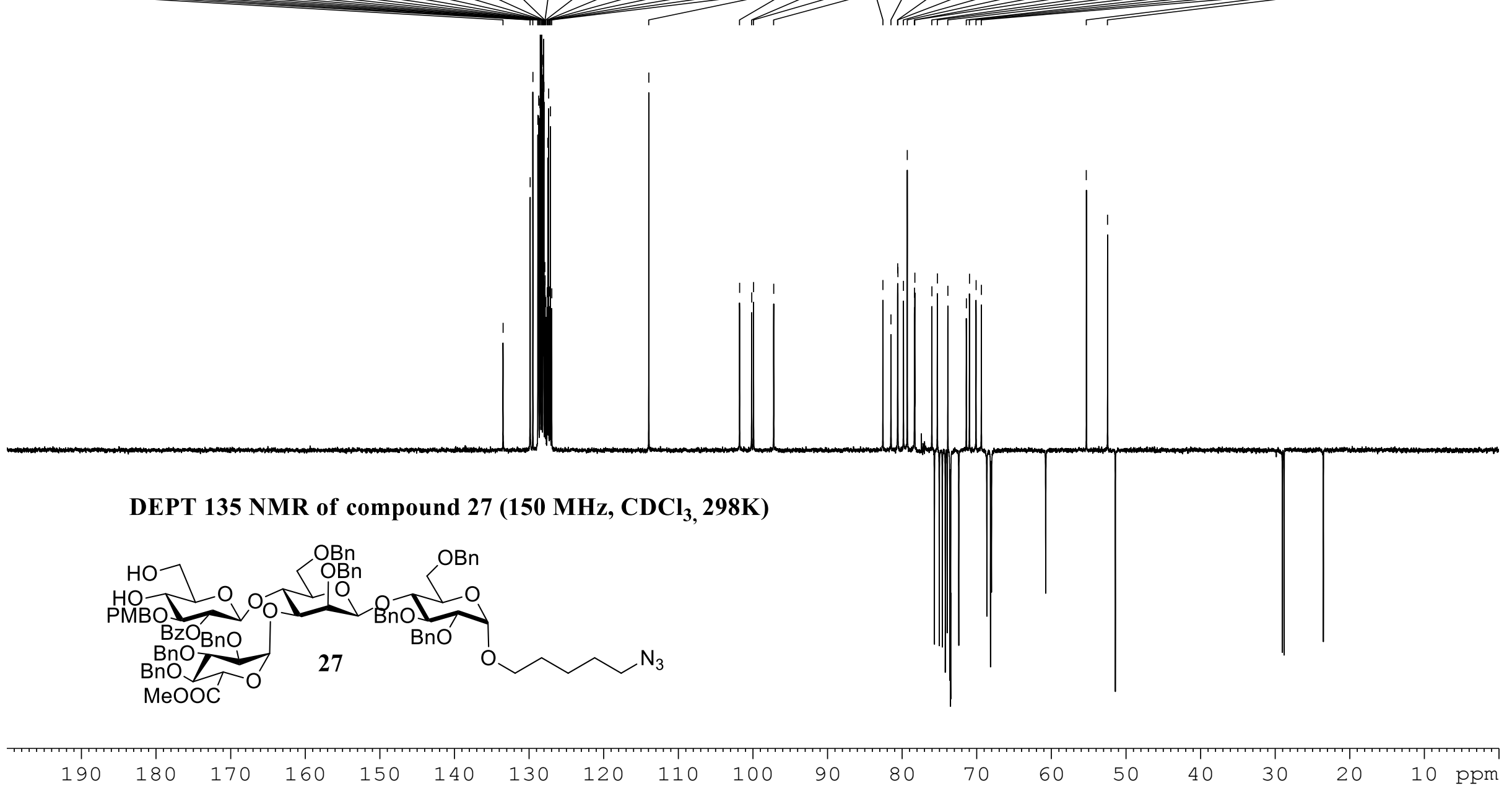




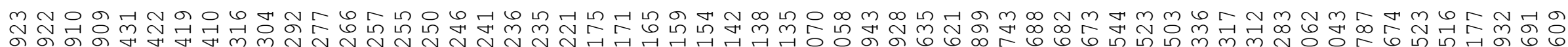

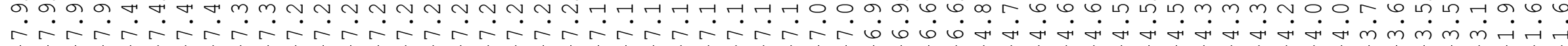

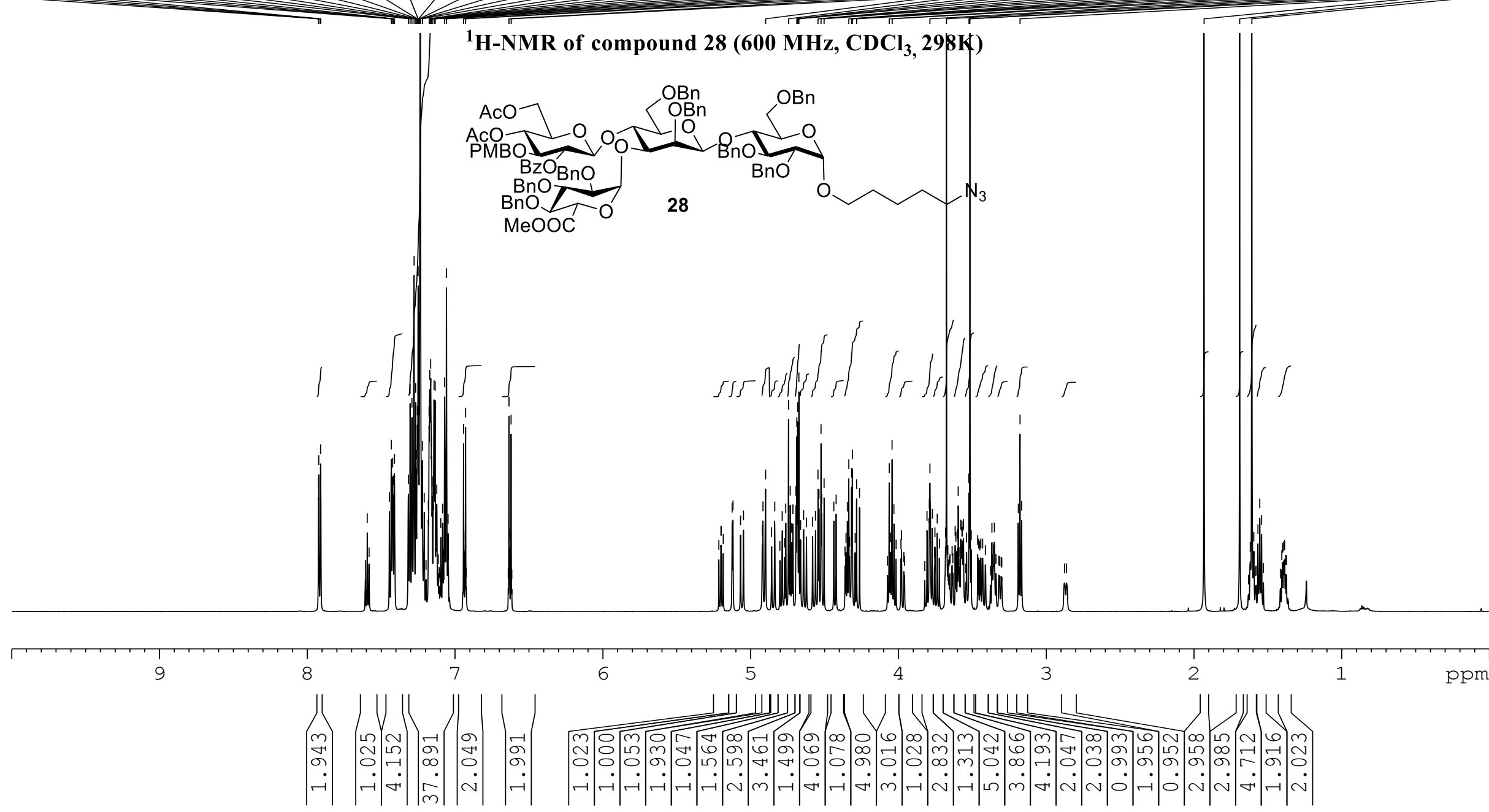




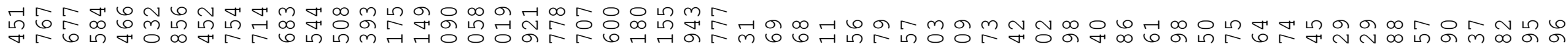

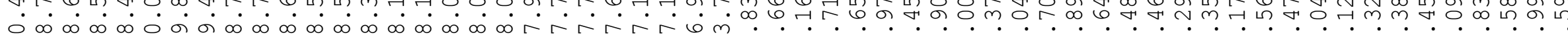

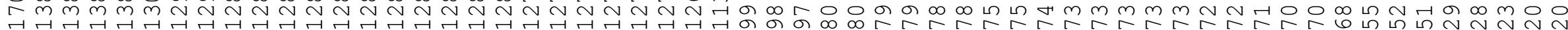

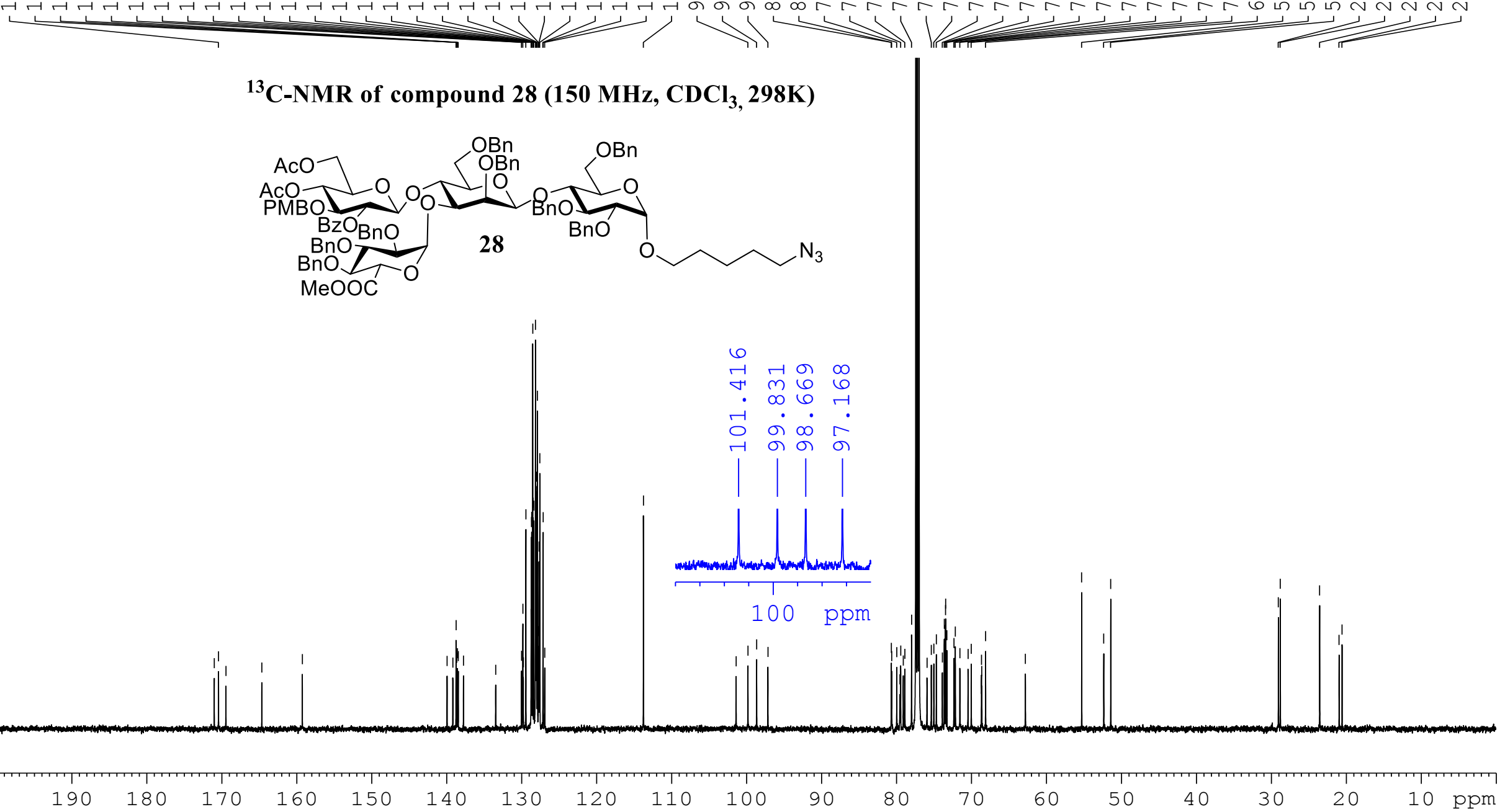




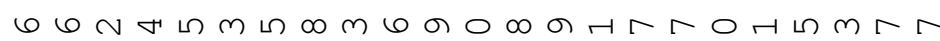

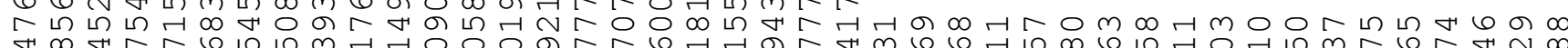

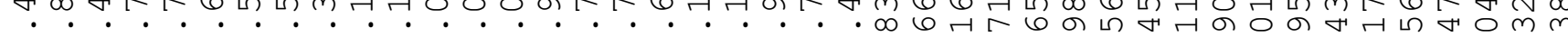

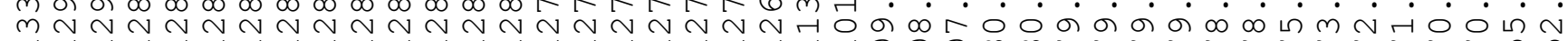

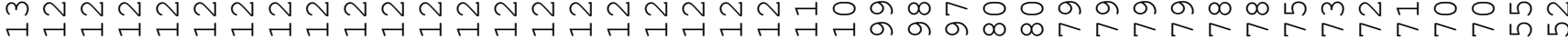

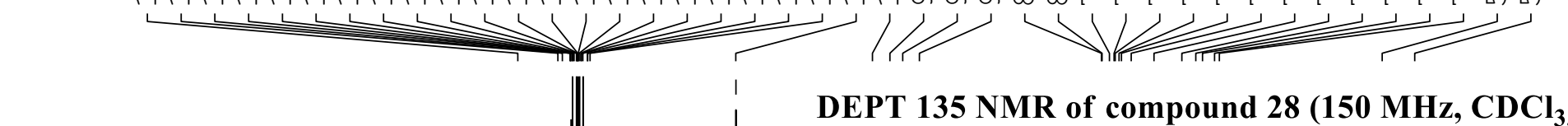

DEPT 135 NMR of compound $28\left(150 \mathrm{MHz}, \mathrm{CDCl}_{3}, 298 \mathrm{~K}\right)$
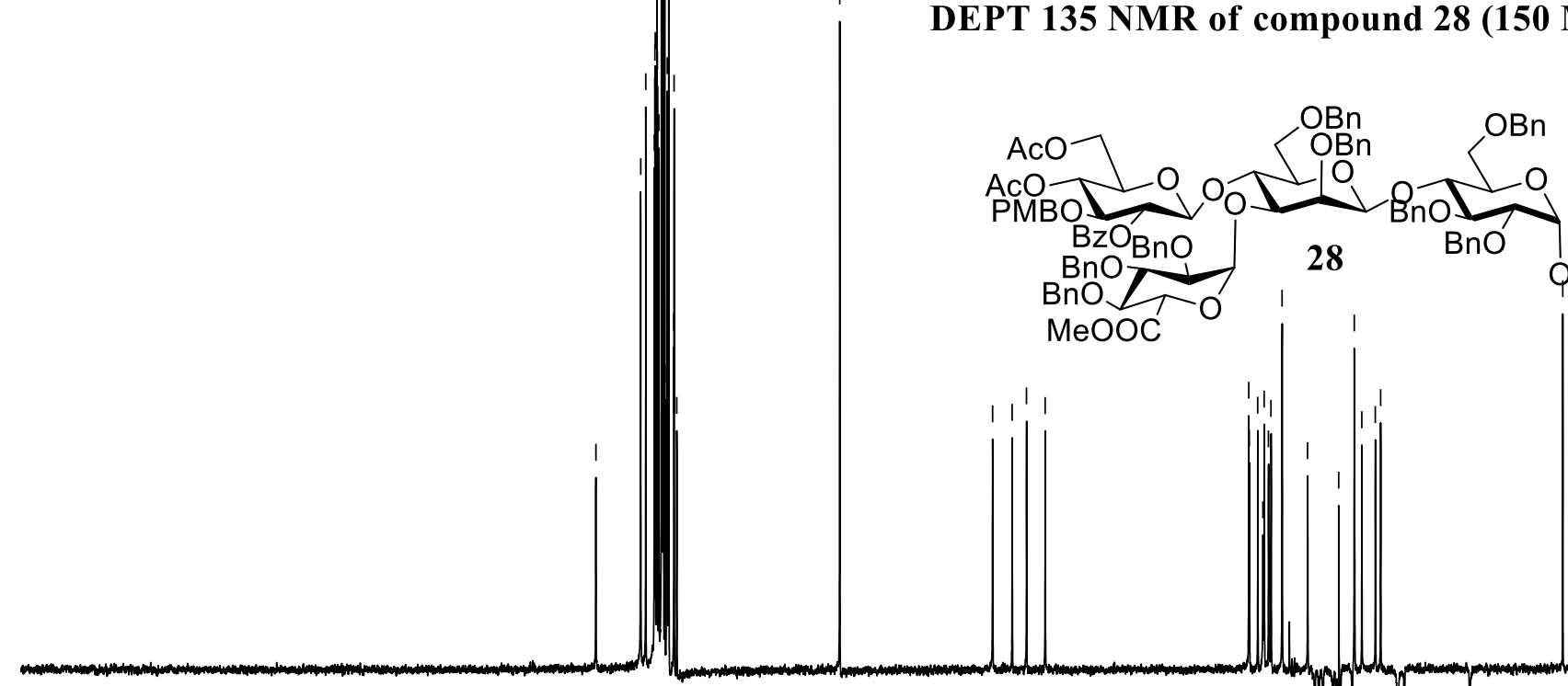

28

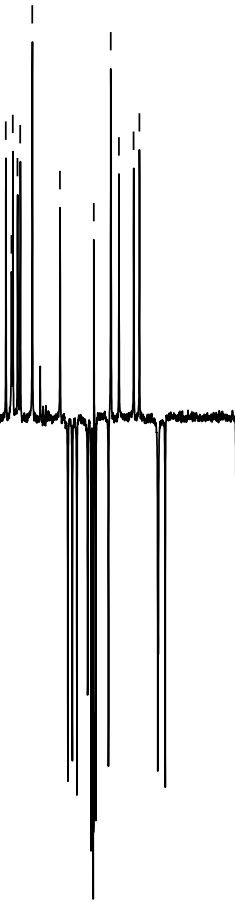

P次 $\mathrm{N}_{3}$

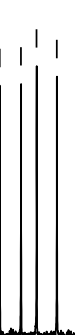

S44 


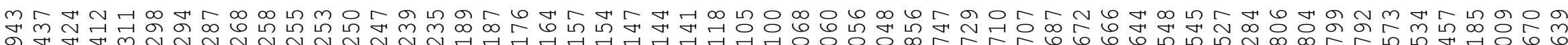

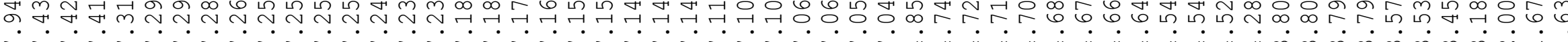

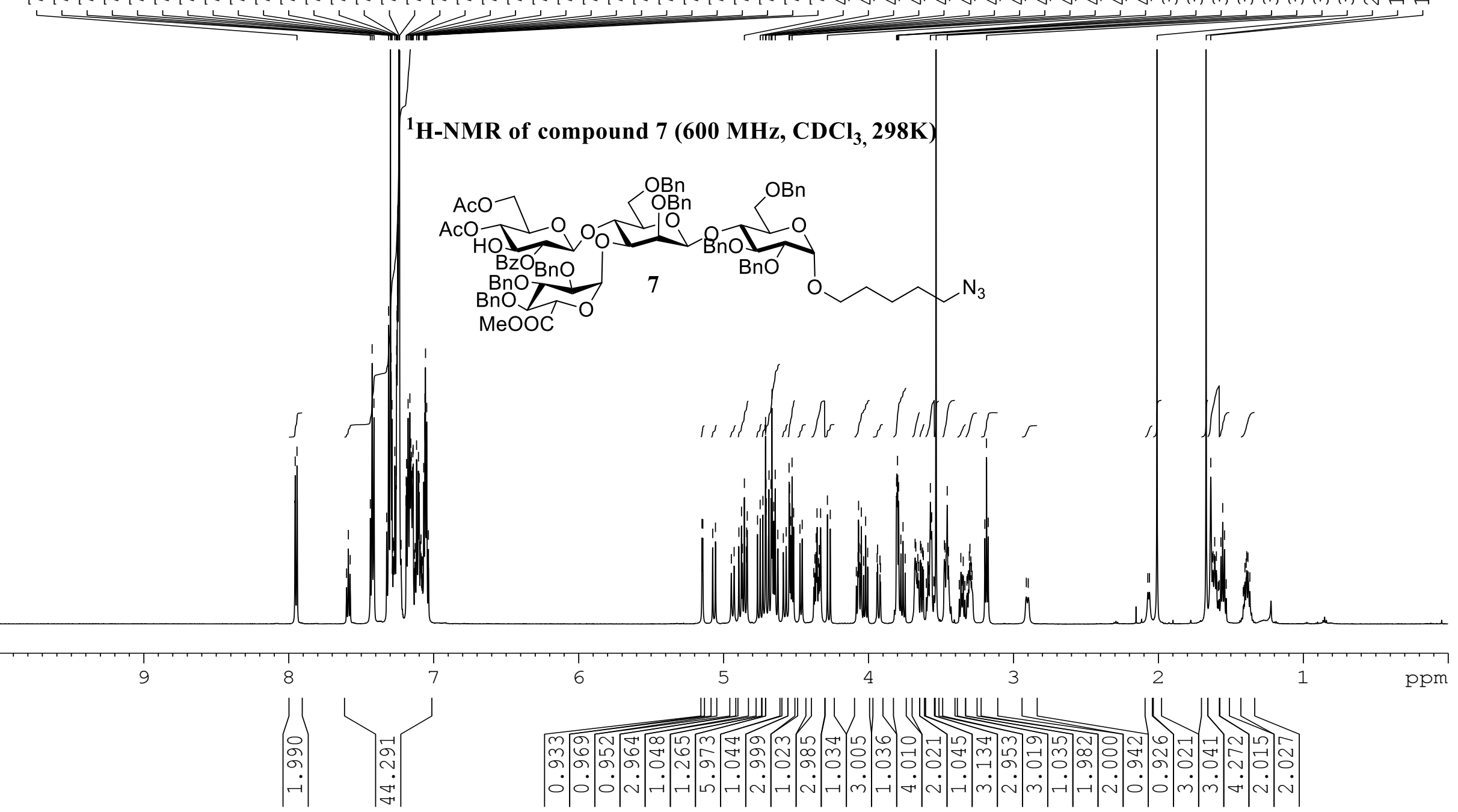




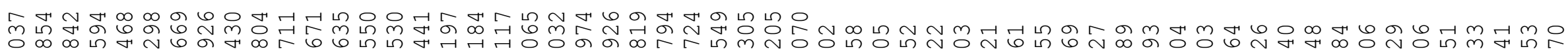

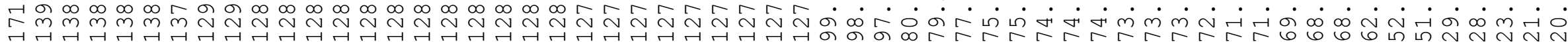

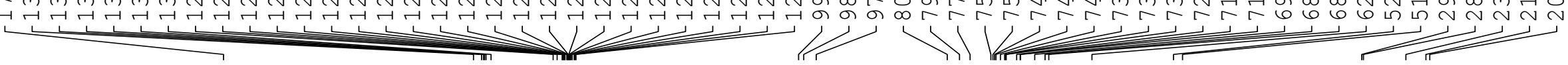

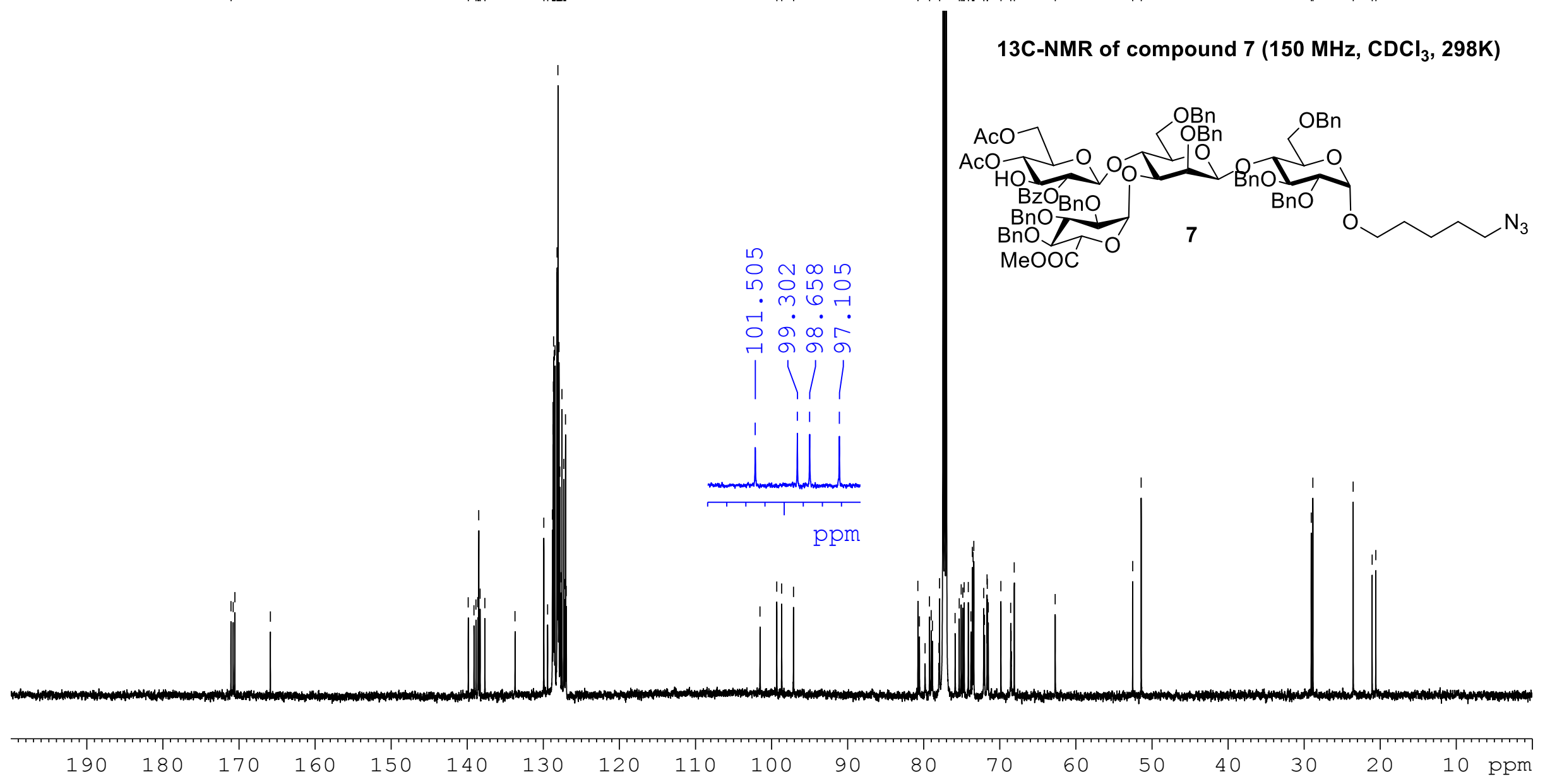


H

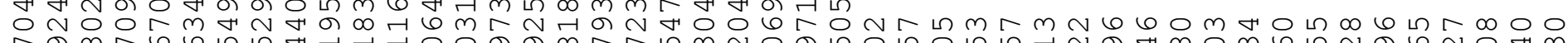

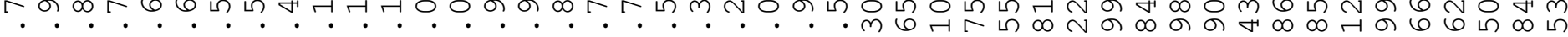

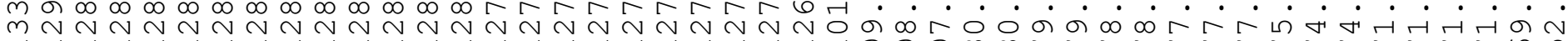

H-H

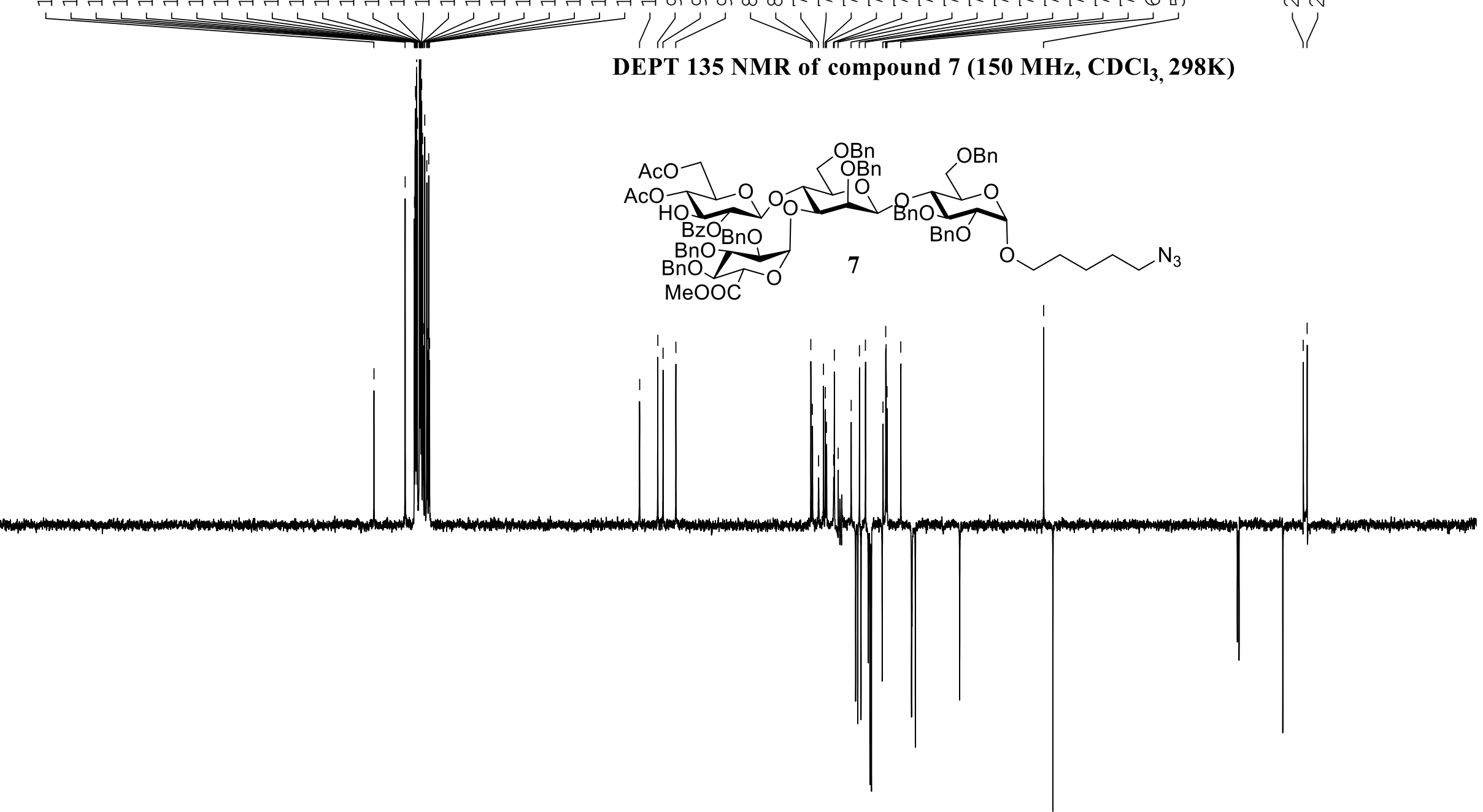

S47 
능

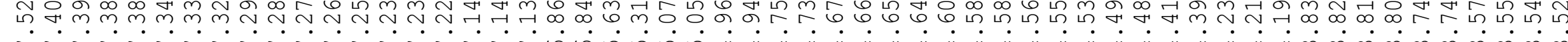

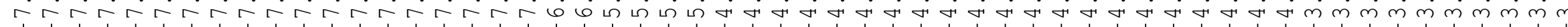

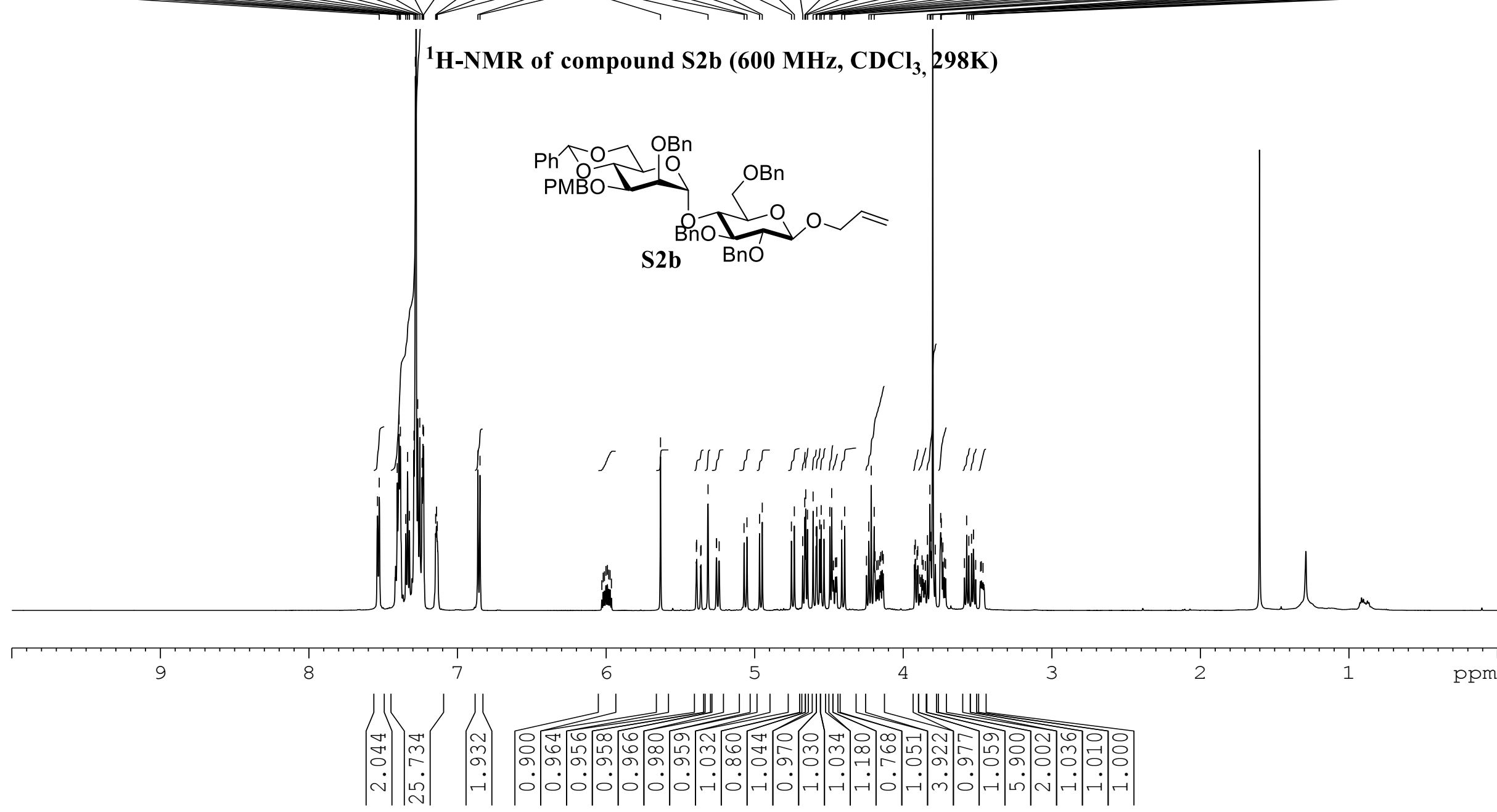




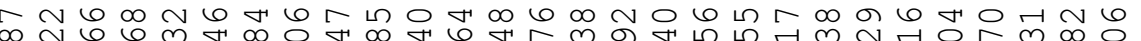

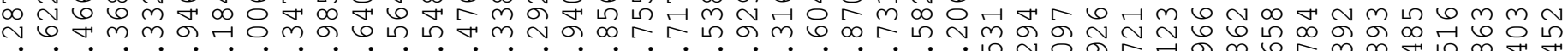

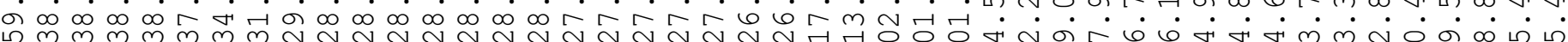

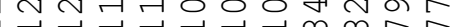

r

${ }^{13} \mathrm{C}-\mathrm{NMR}$ of compound $\mathrm{S2b}\left(150 \mathrm{MHz}, \mathrm{CDCl}_{3}, 298 \mathrm{~K}\right)$
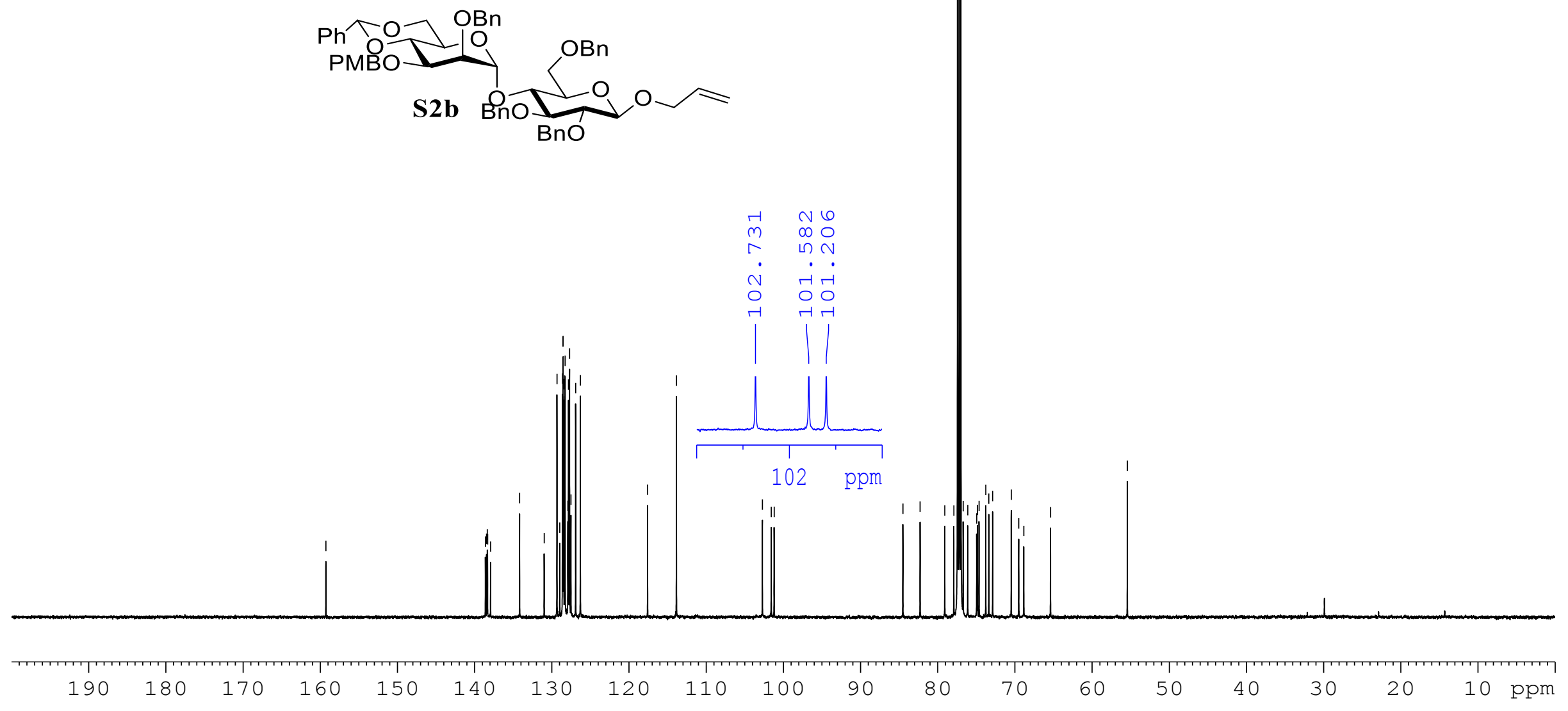


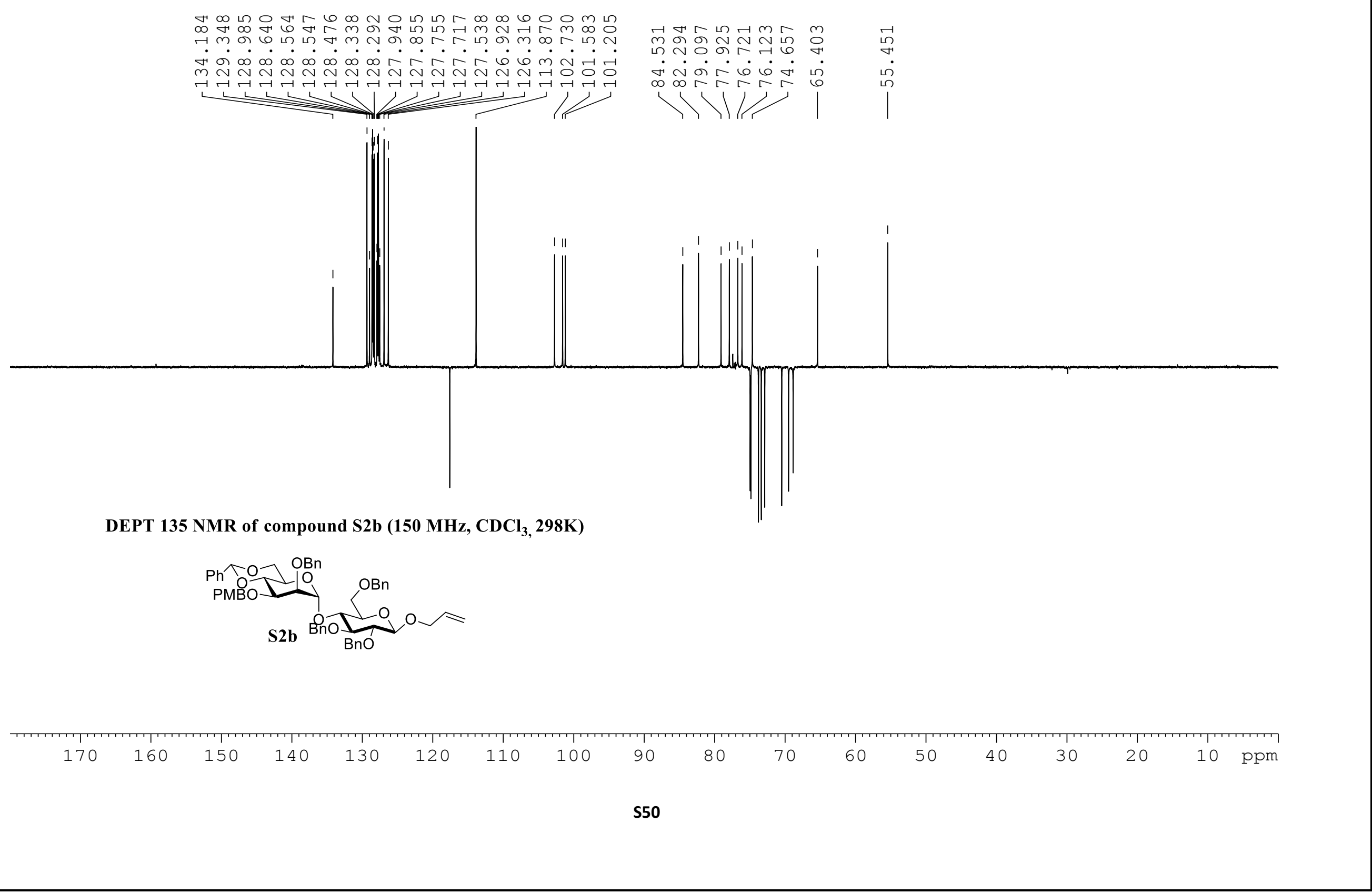




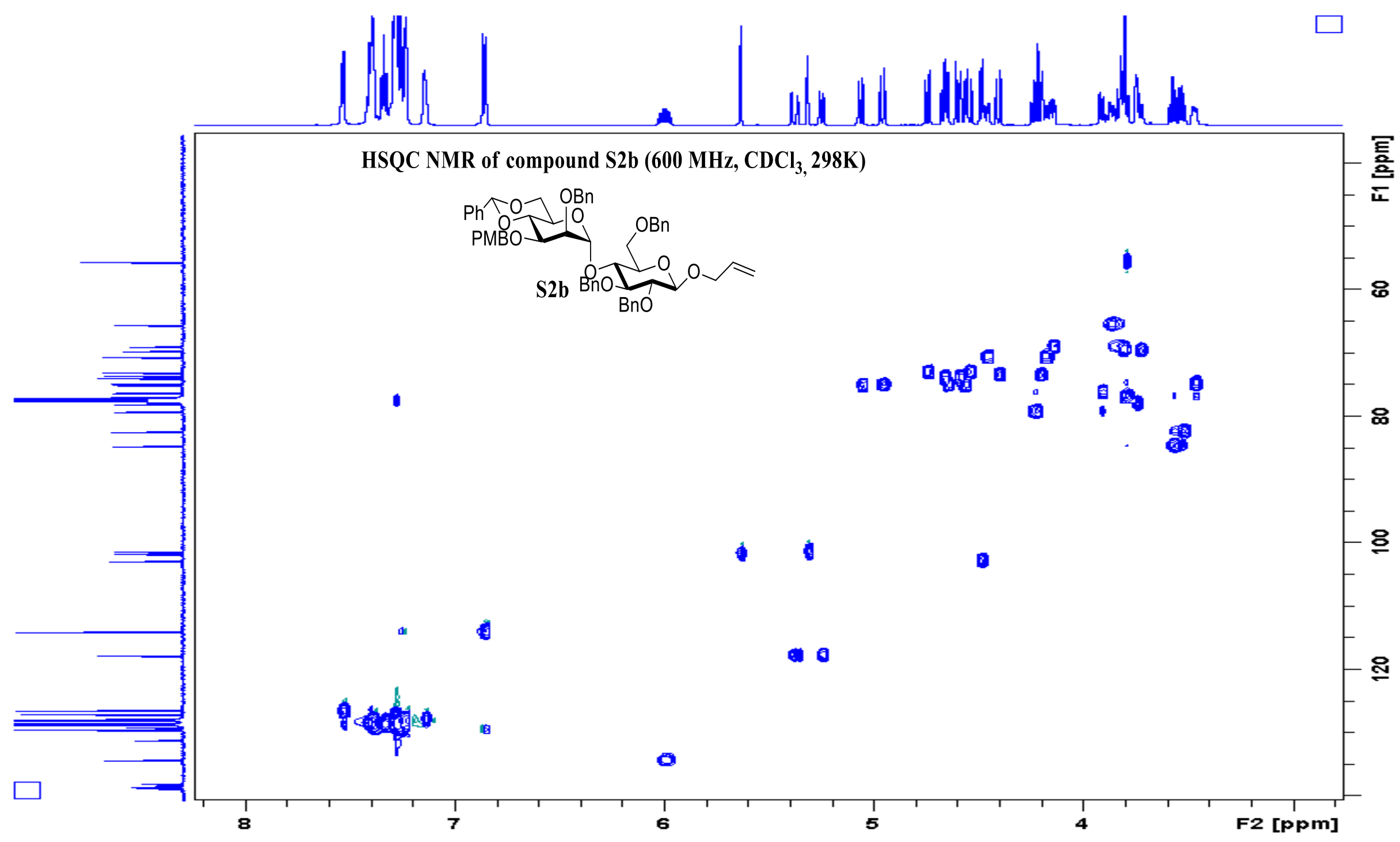




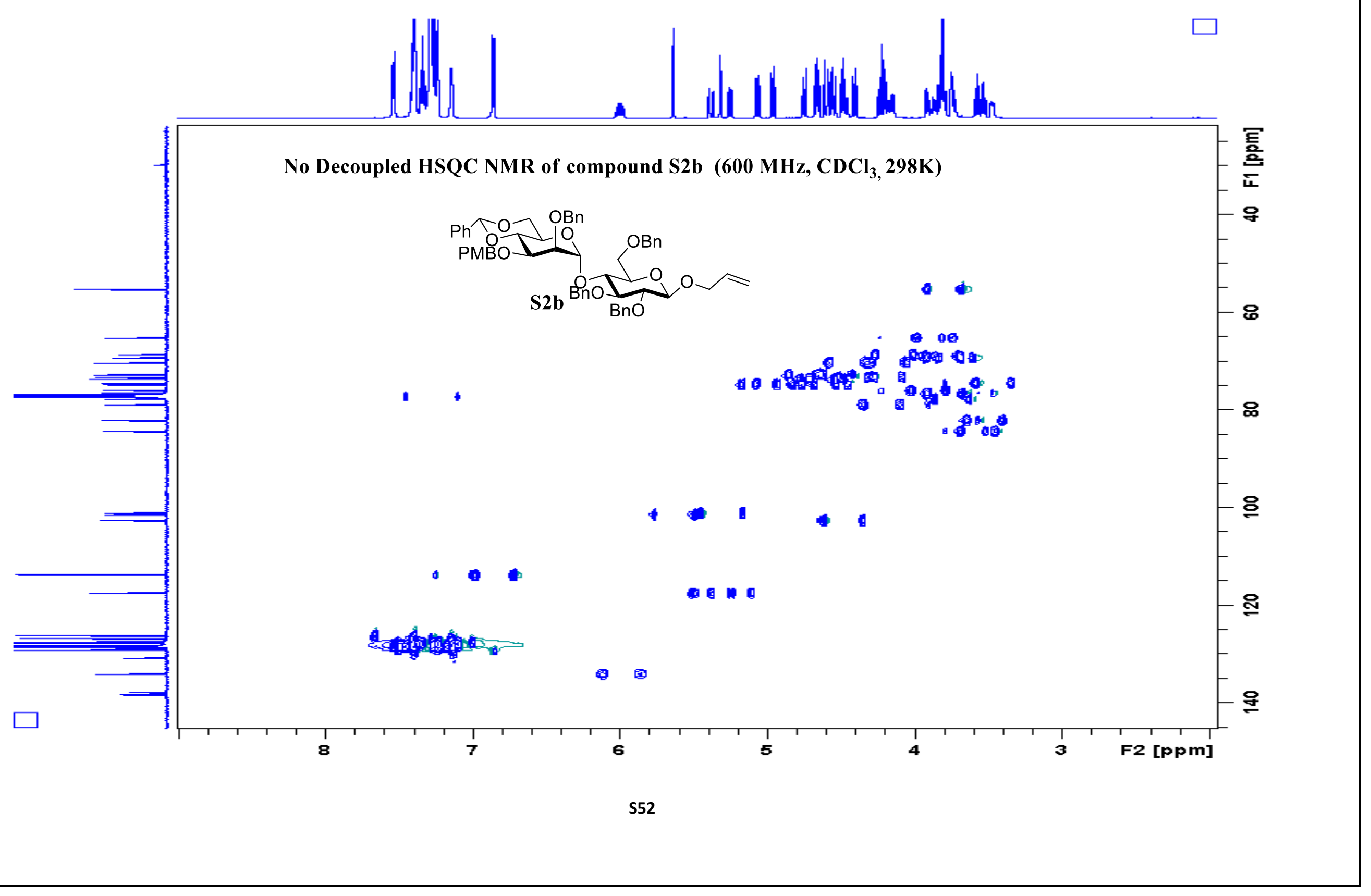




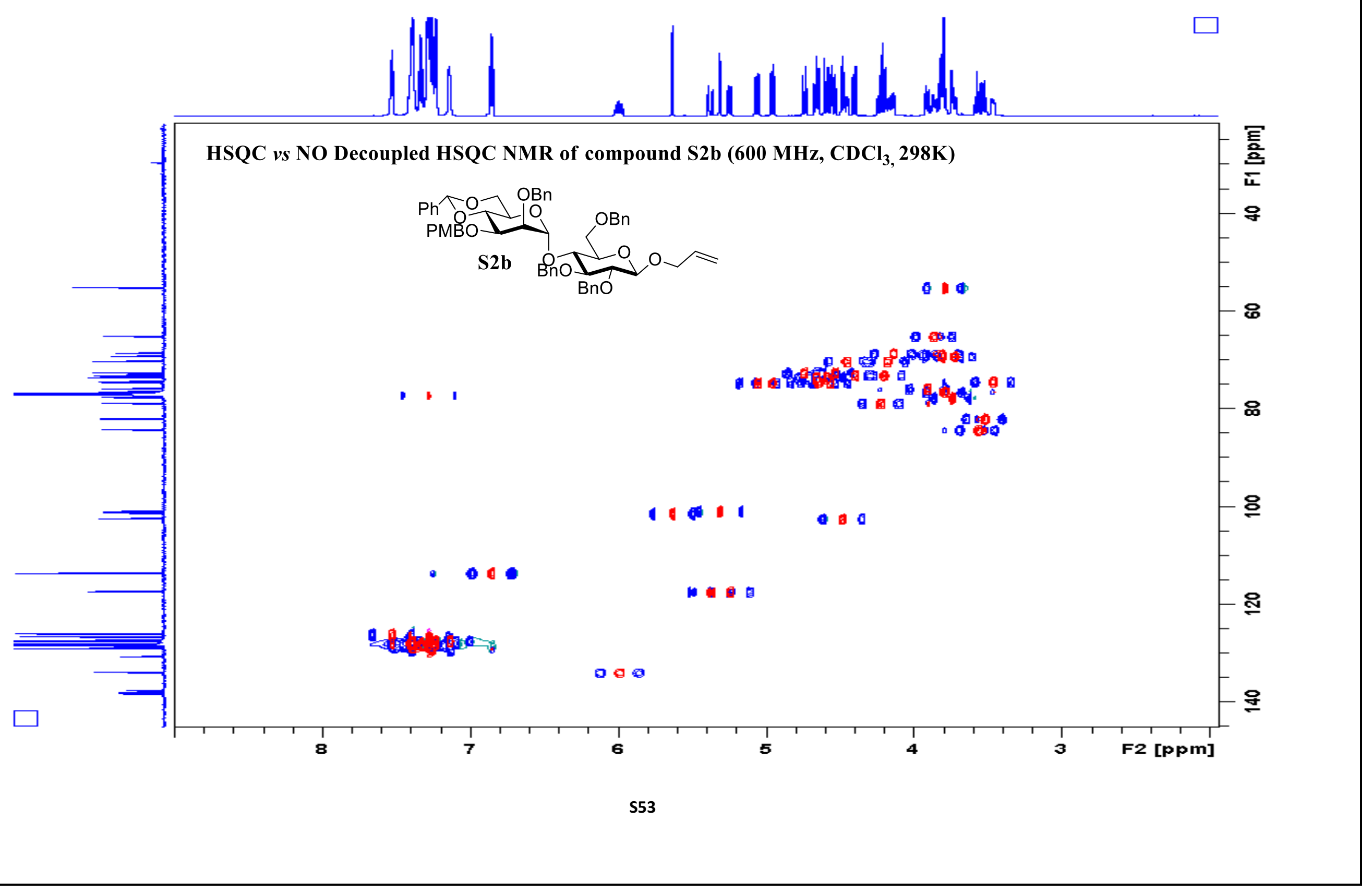




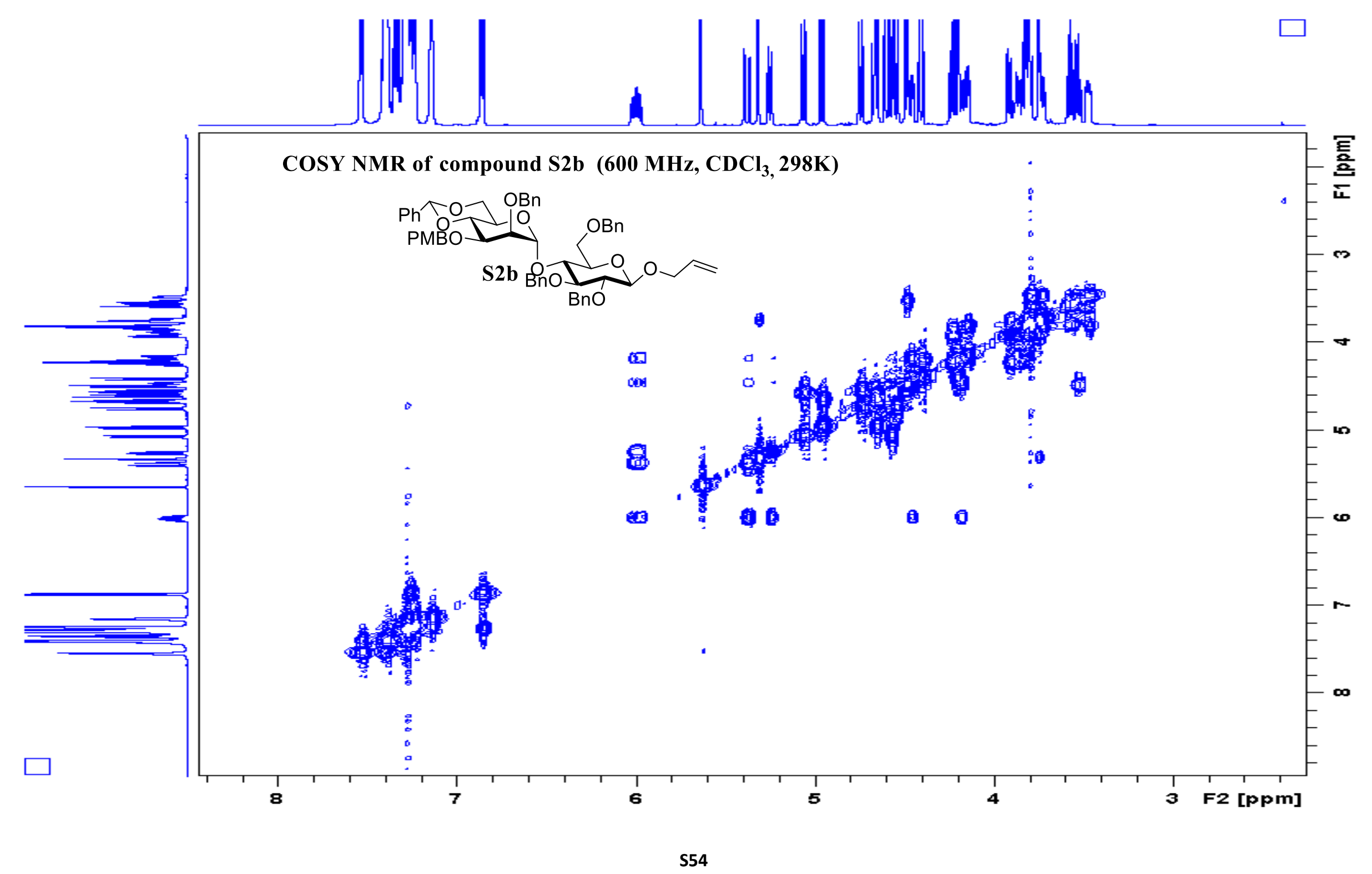




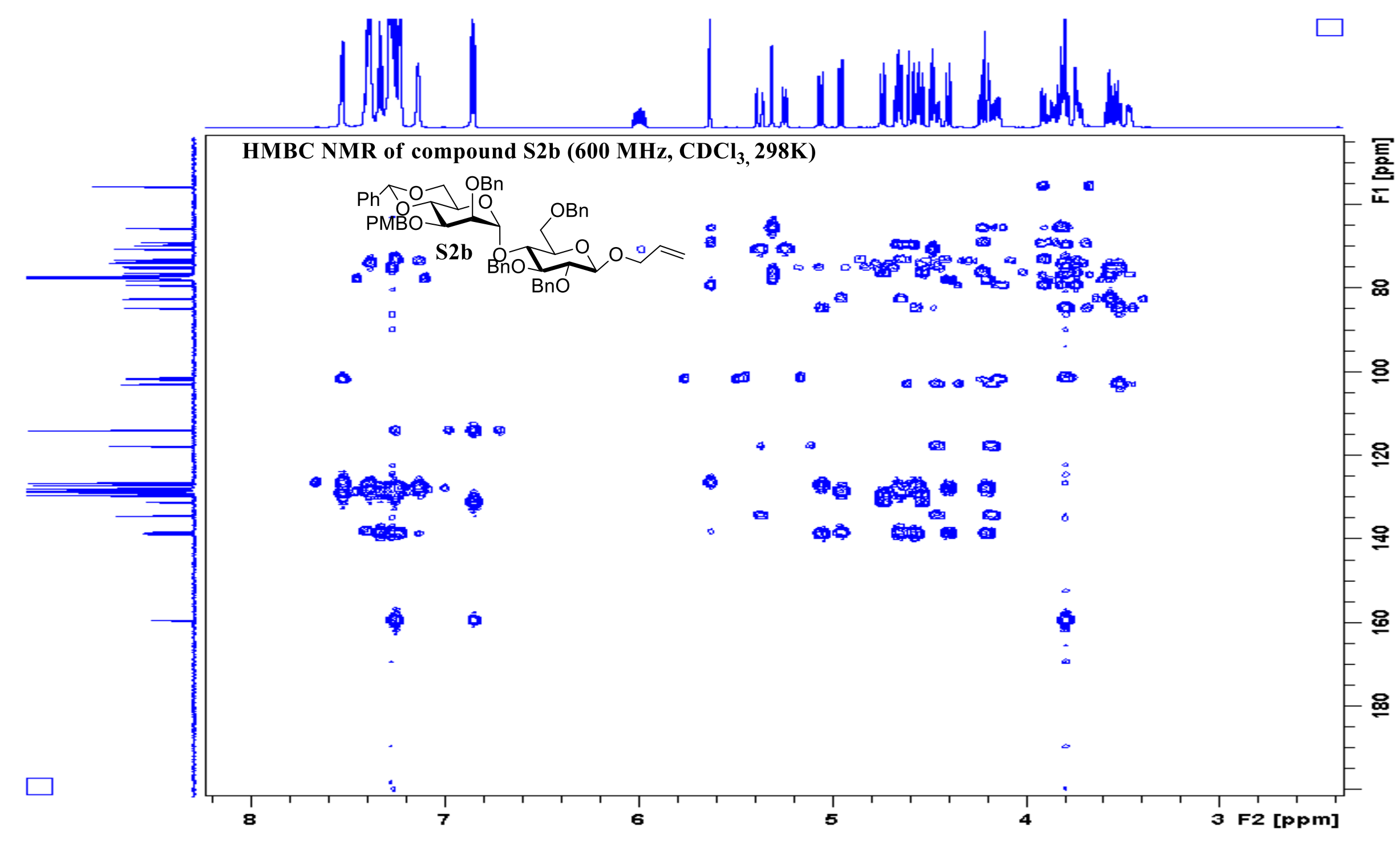




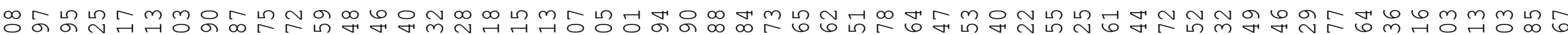

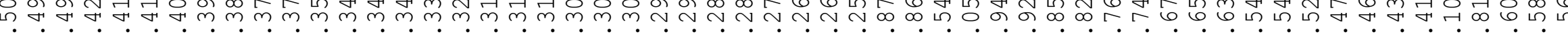

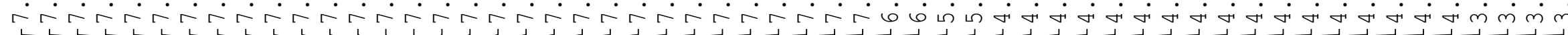

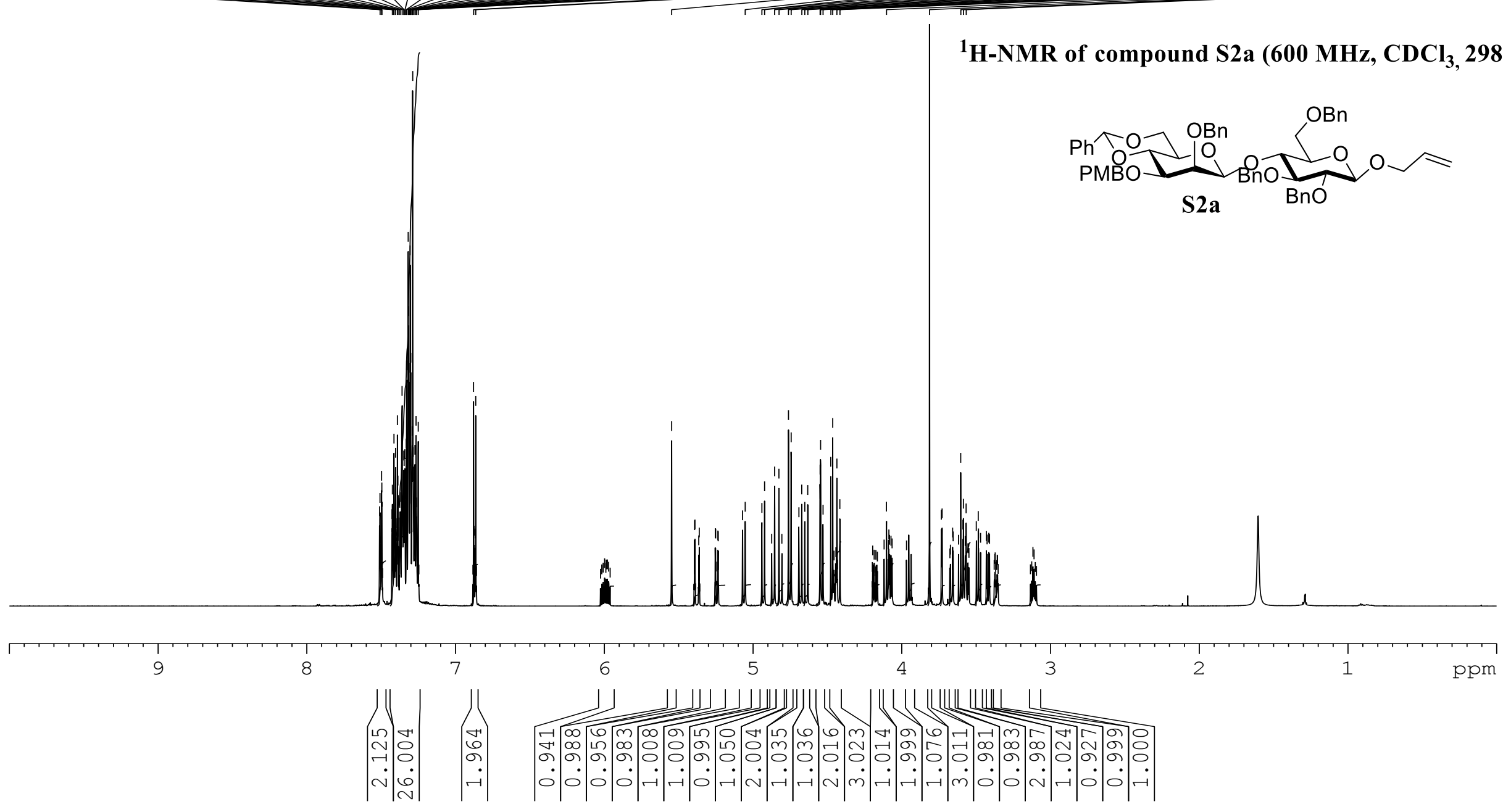


꾼

mm $m$ o 00 N

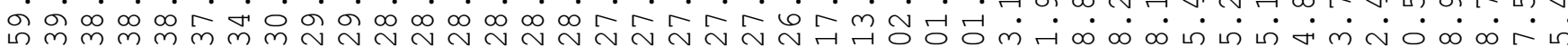

${ }^{13} \mathrm{C}-\mathrm{NMR}$ of compound $\mathrm{S2a}\left(150 \mathrm{MHz}, \mathrm{CDCl}_{3}, 298 \mathrm{~K}\right)$

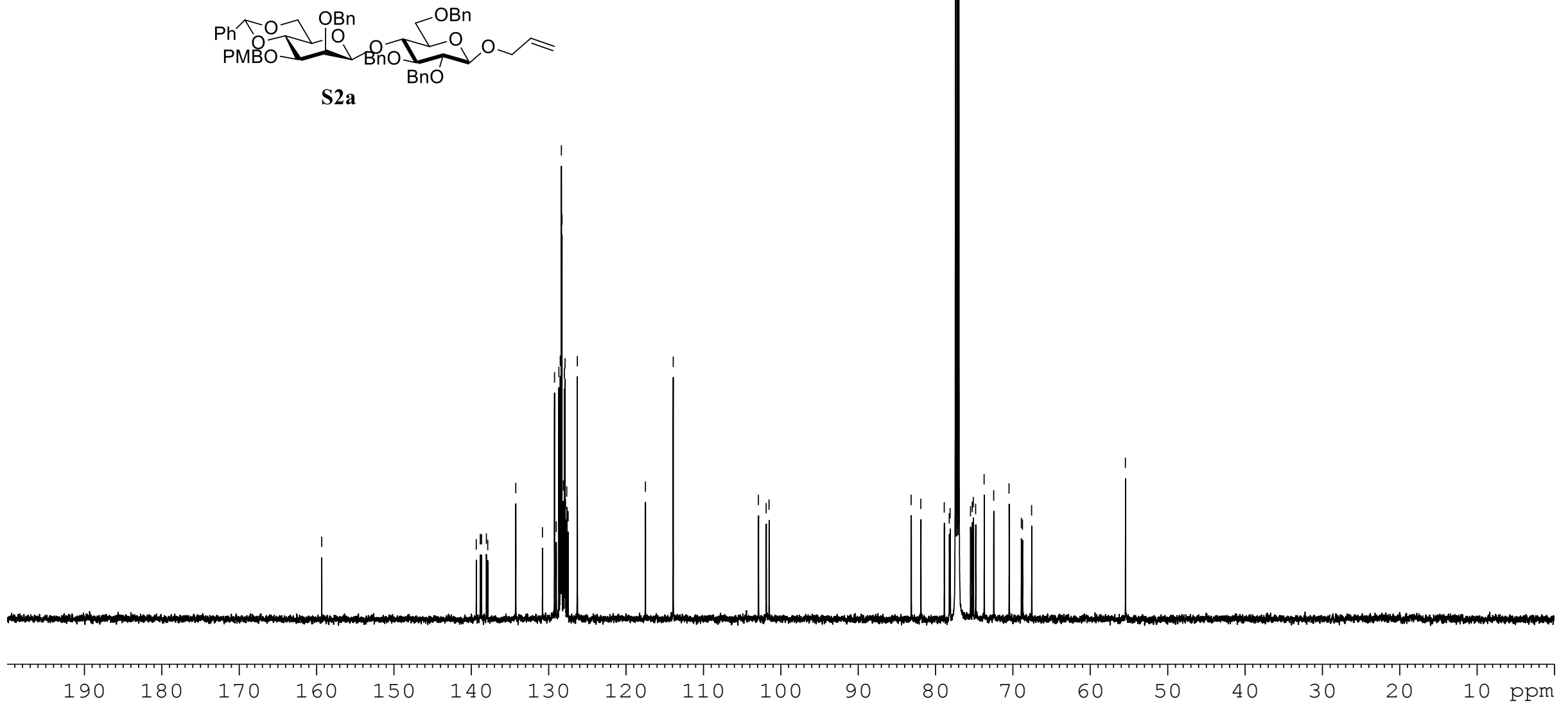

S2a

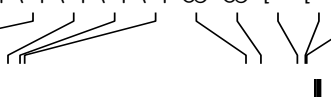

S57 


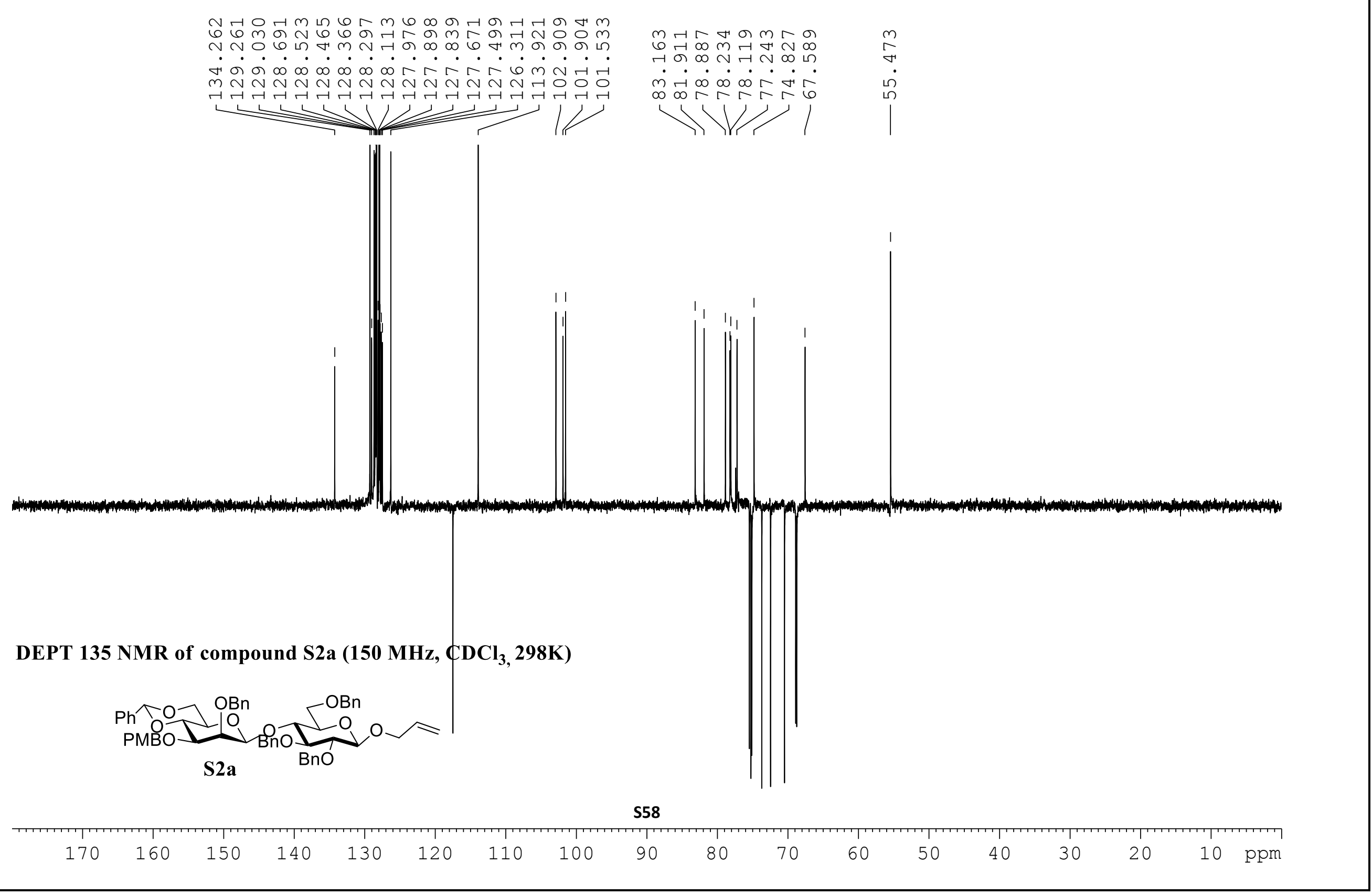




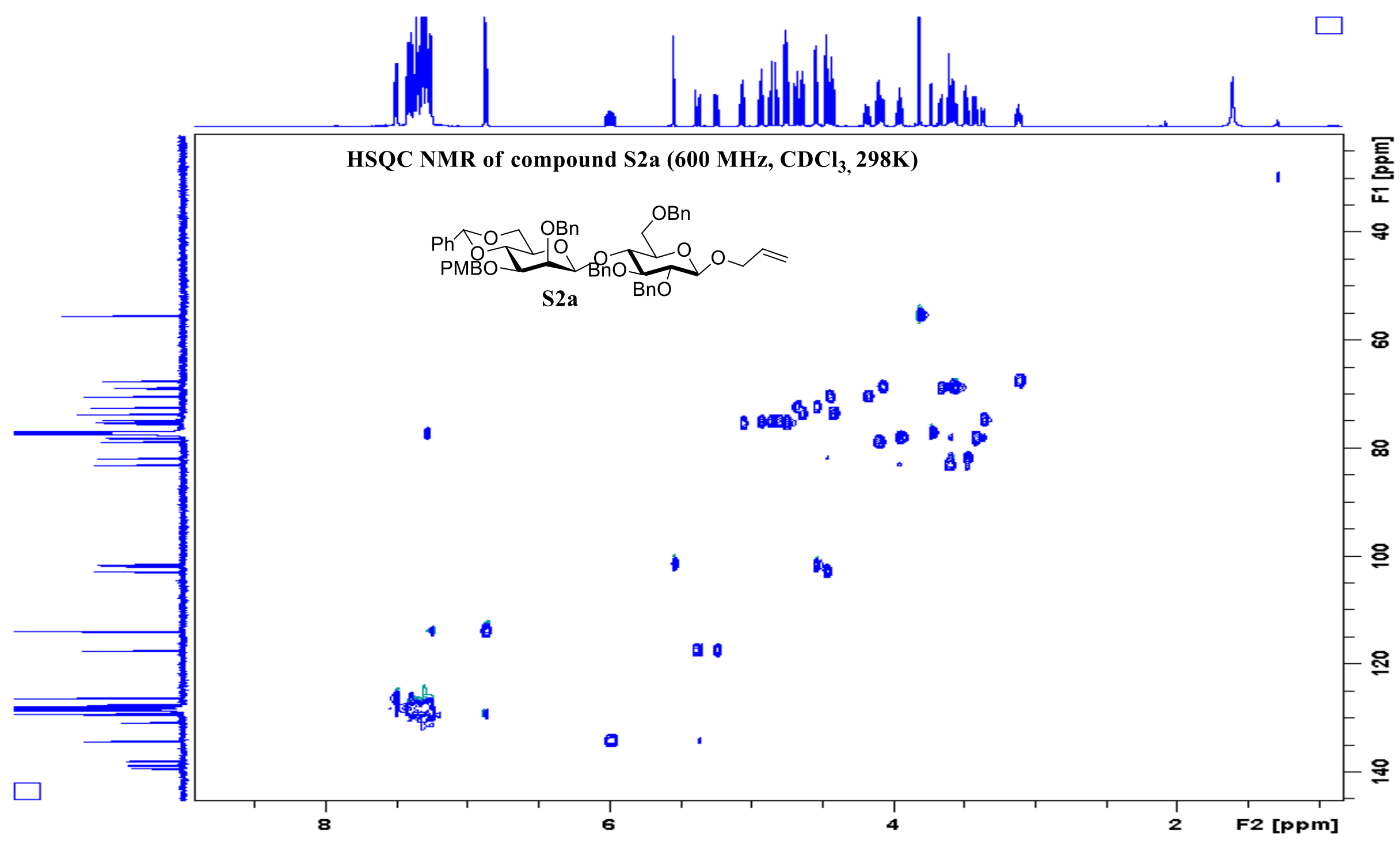




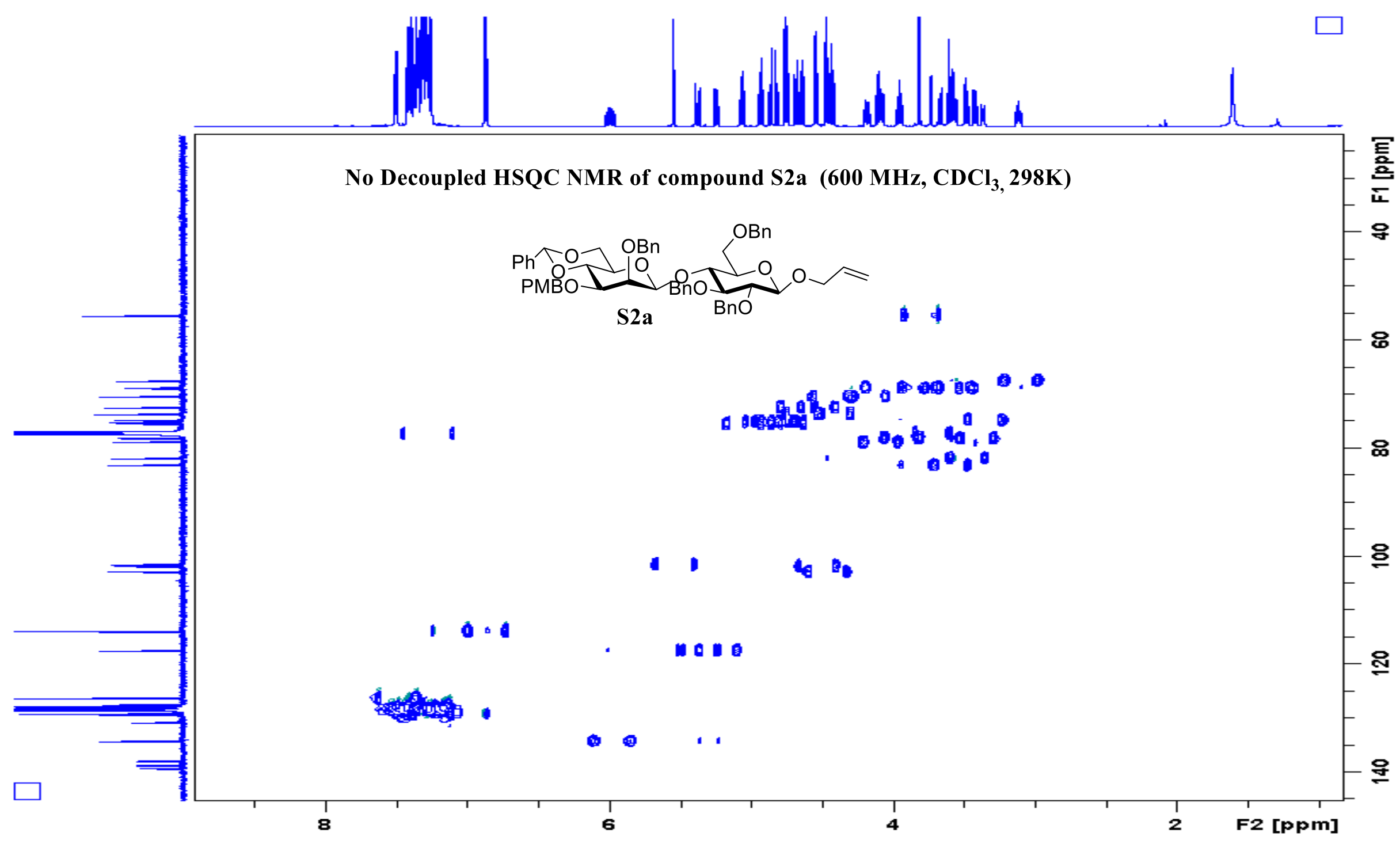




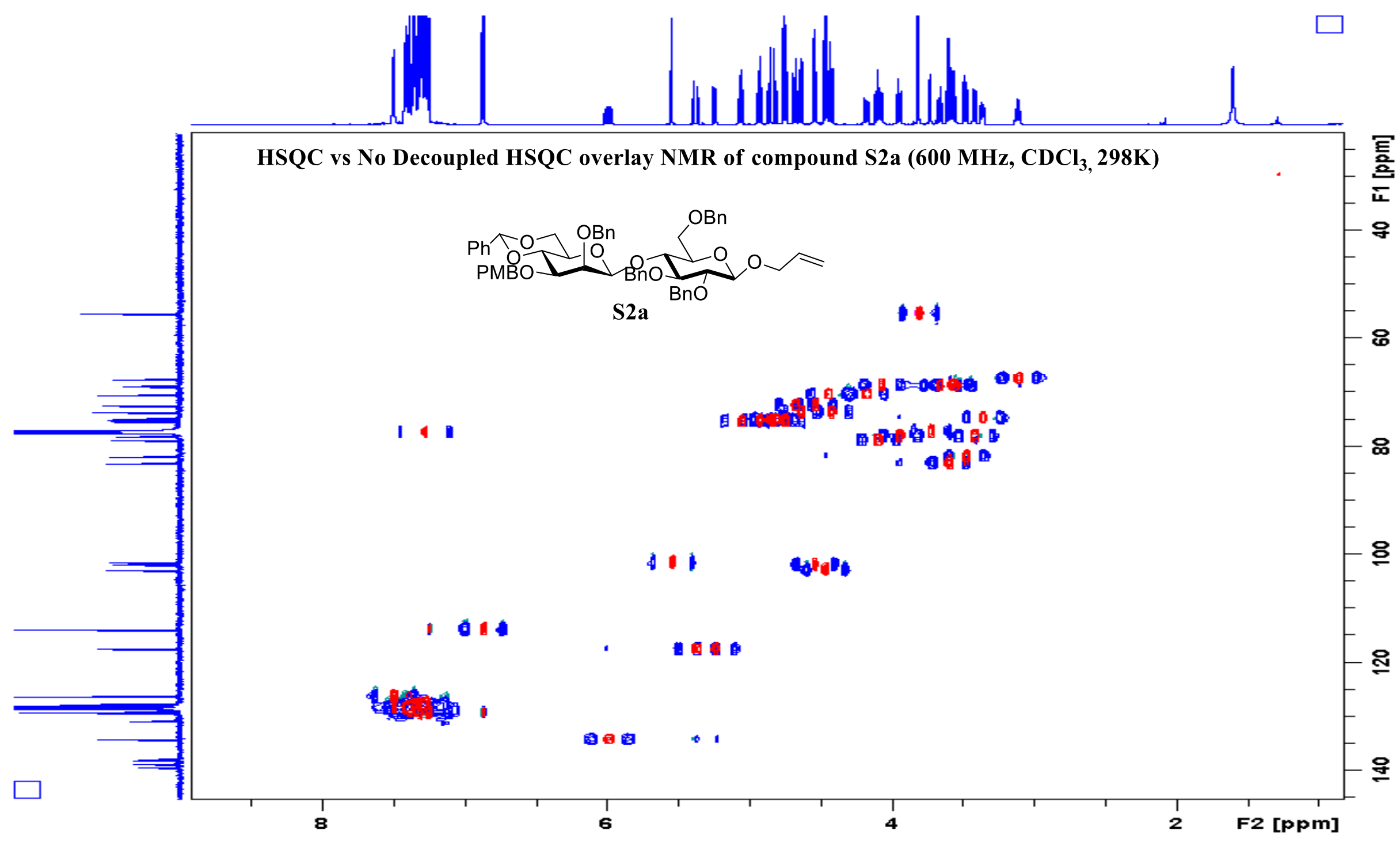

561 


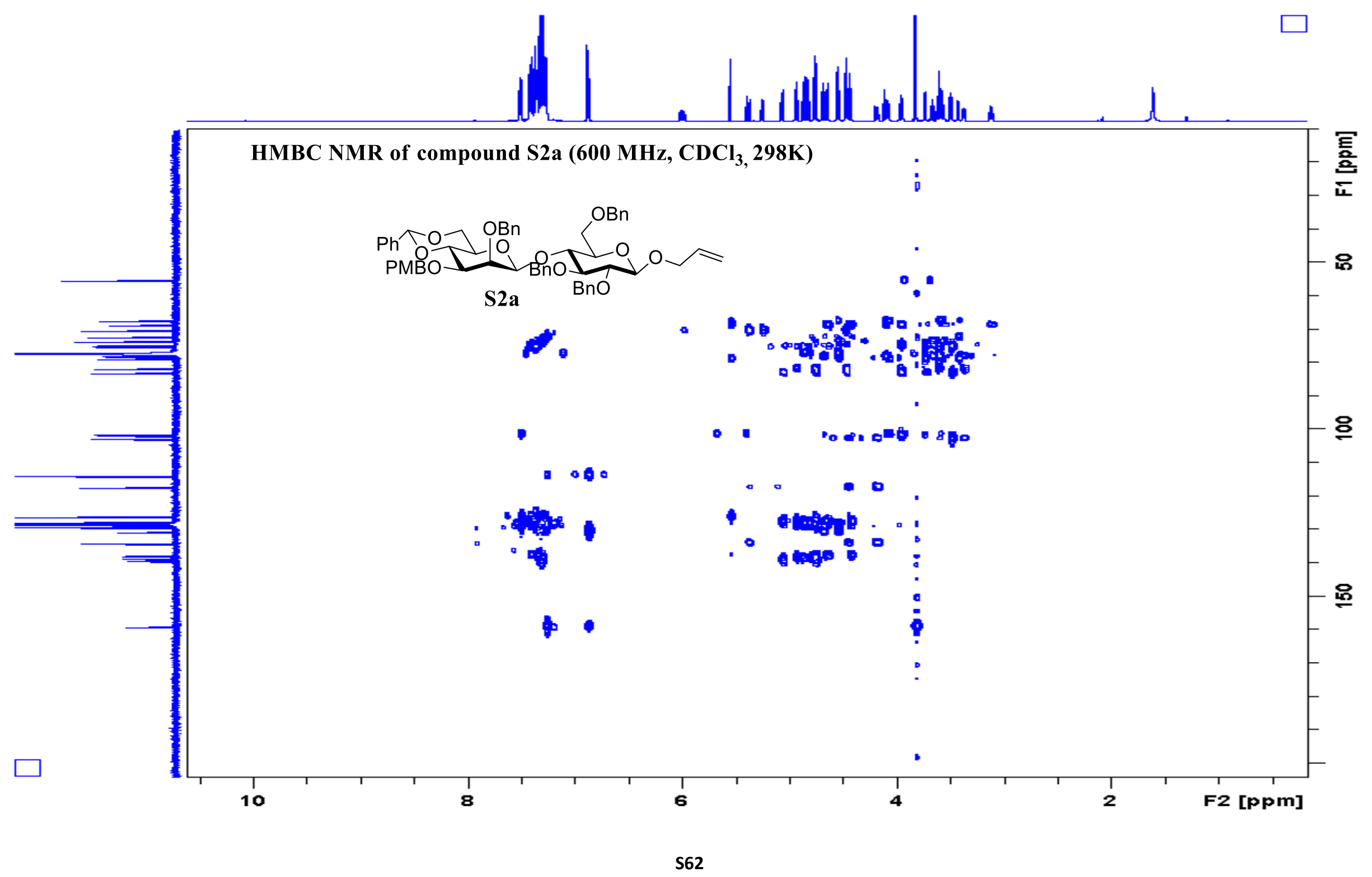




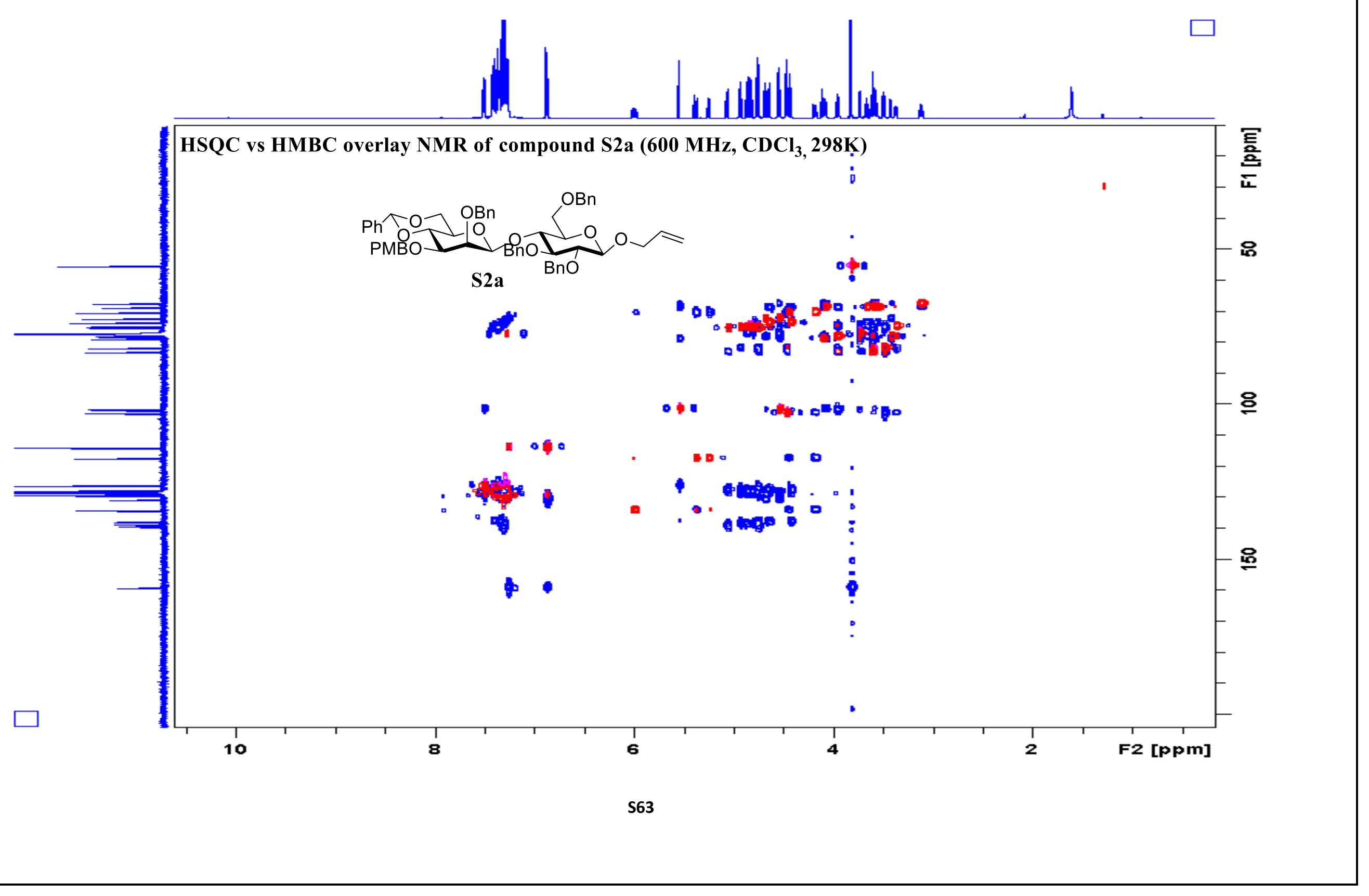




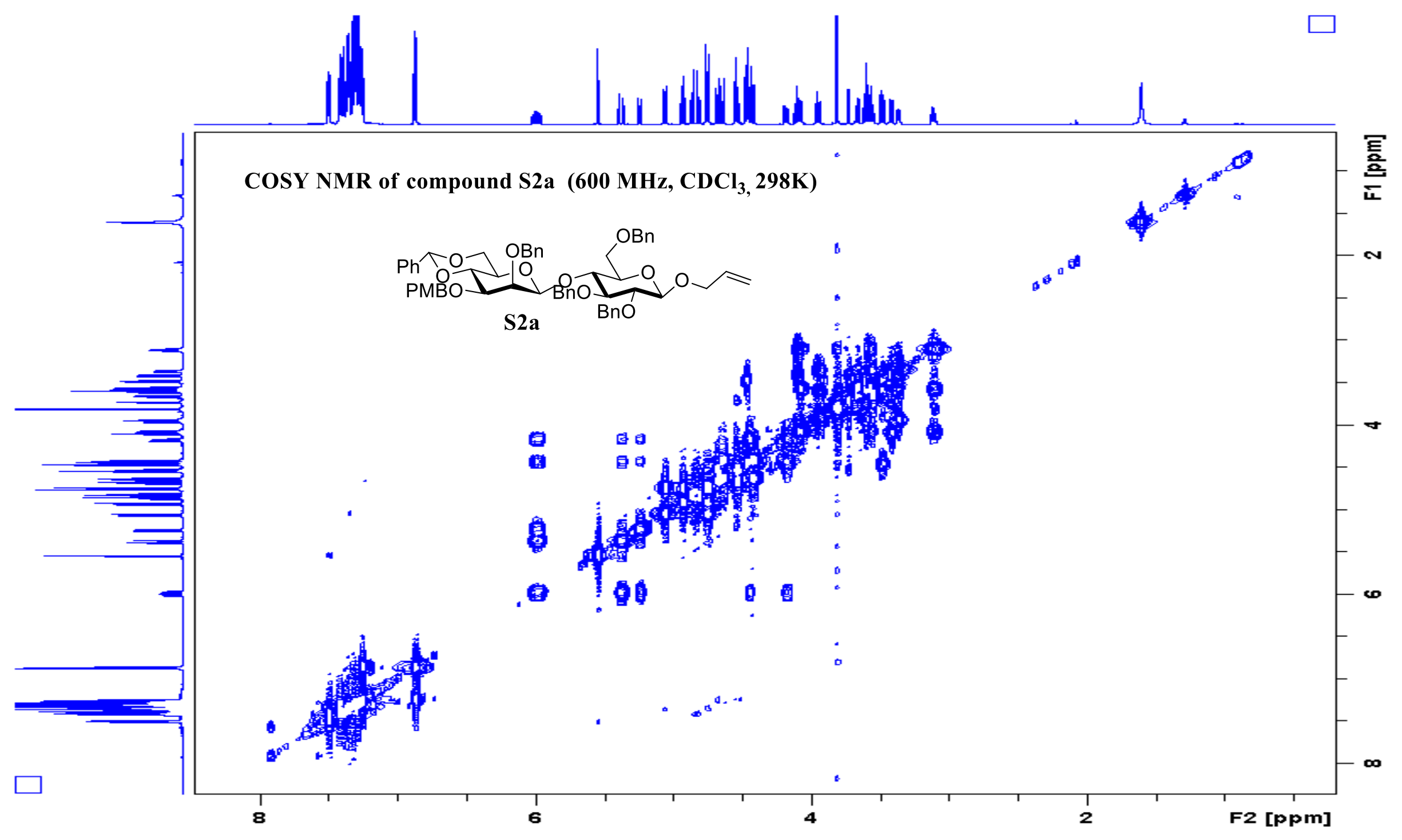


임

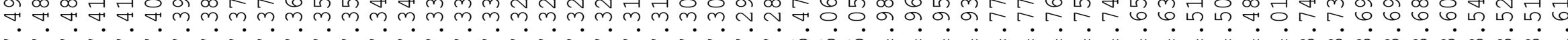

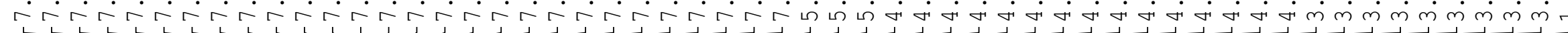

${ }^{1} \mathrm{H}-\mathrm{NMR}$ of compound $\mathrm{S3}\left(600 \mathrm{MHz}, \mathrm{CDCl}_{3}, 298 \mathrm{~K}\right)$
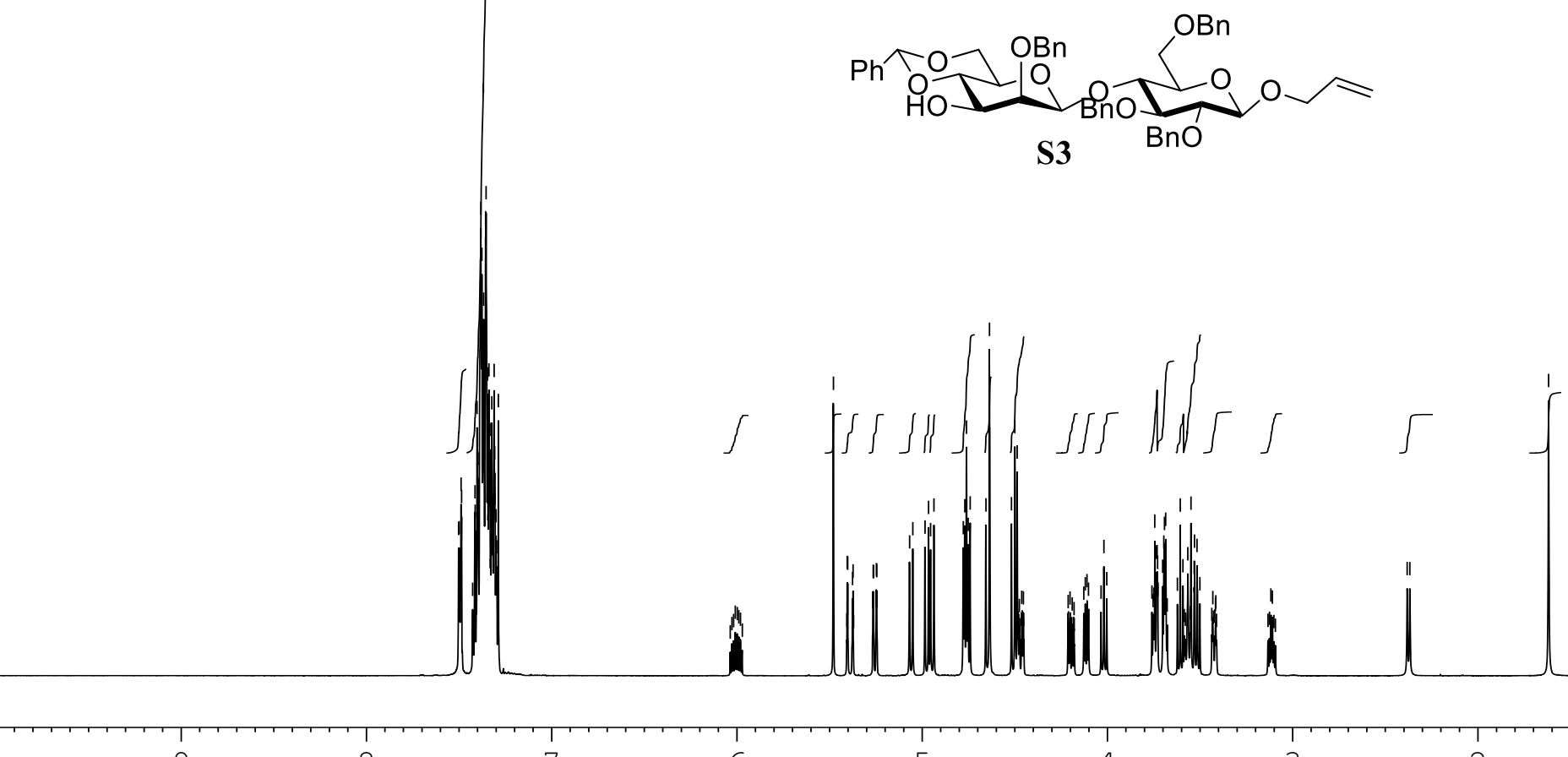

8

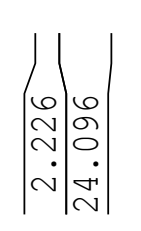

7

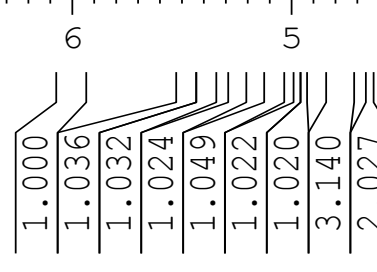

4

3

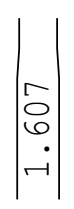




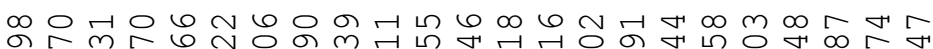

থ $6 m \infty$ m

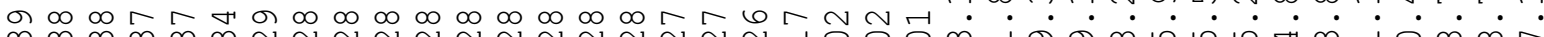

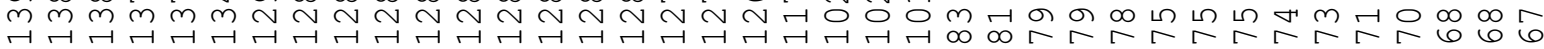

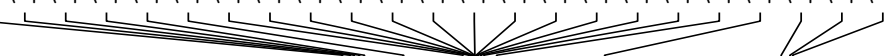

${ }^{13} \mathrm{C}-\mathrm{NMR}$ of compound $\mathrm{S3}\left(150 \mathrm{MHz}, \mathrm{CDCl}_{3}, 298 \mathrm{~K}\right)$

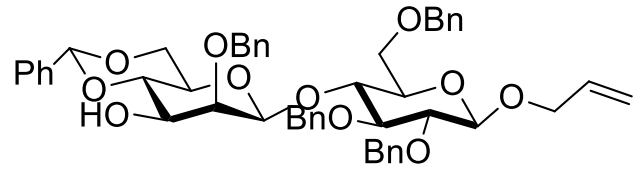

S3

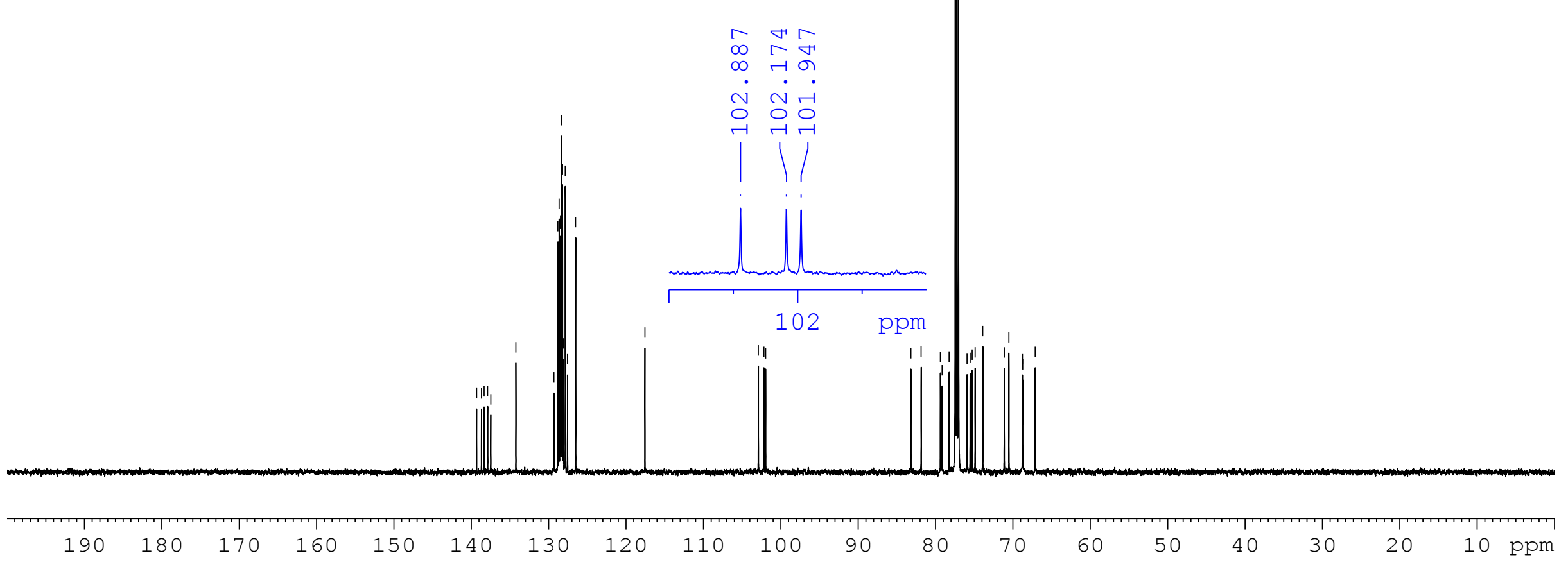




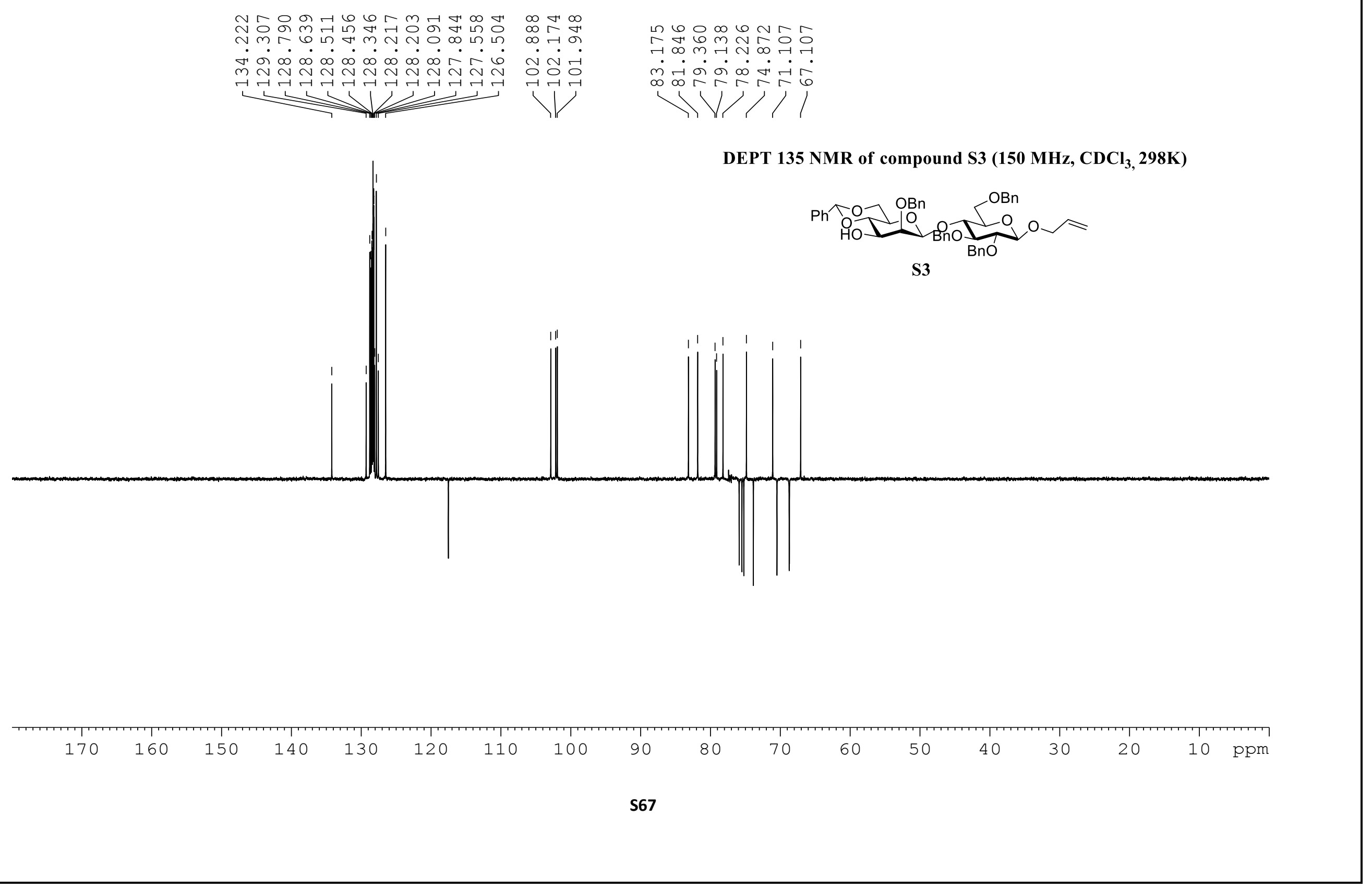




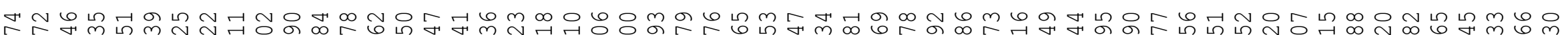
๑.

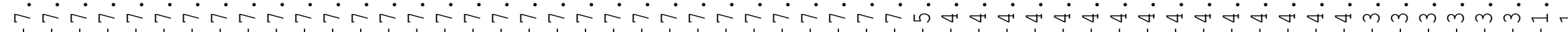

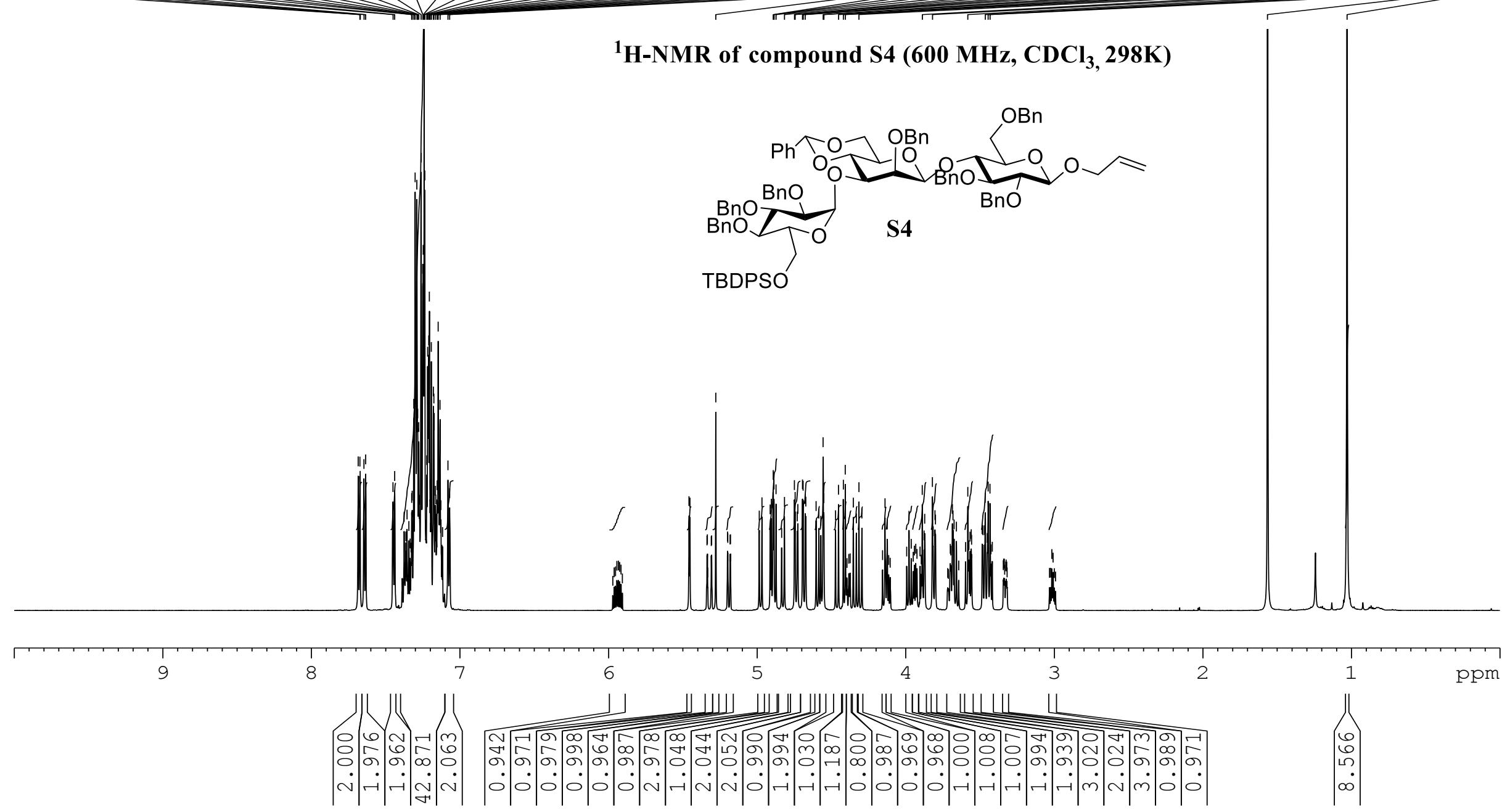




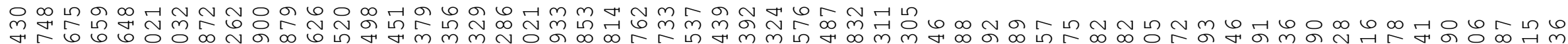

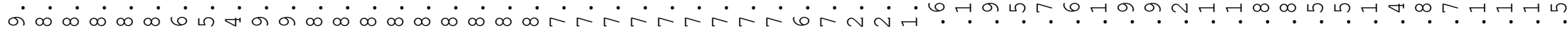

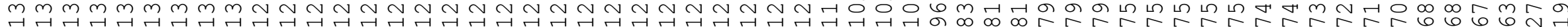

${ }^{13} \mathrm{C}-\mathrm{NMR}$ of compound $\mathrm{S} 4\left(150 \mathrm{MHz}, \mathrm{CDCl}_{3}, 298 \mathrm{~K}\right)$
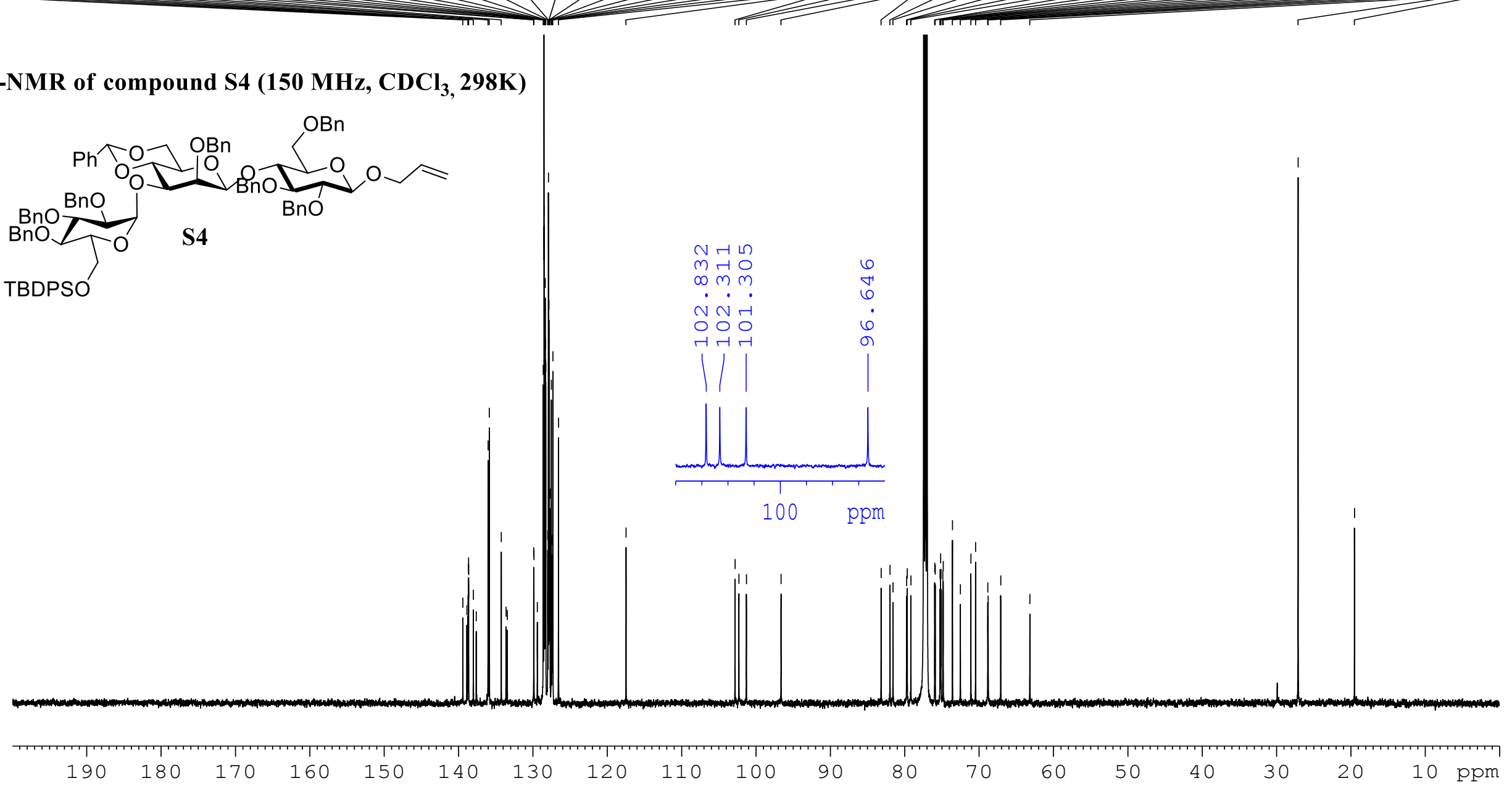

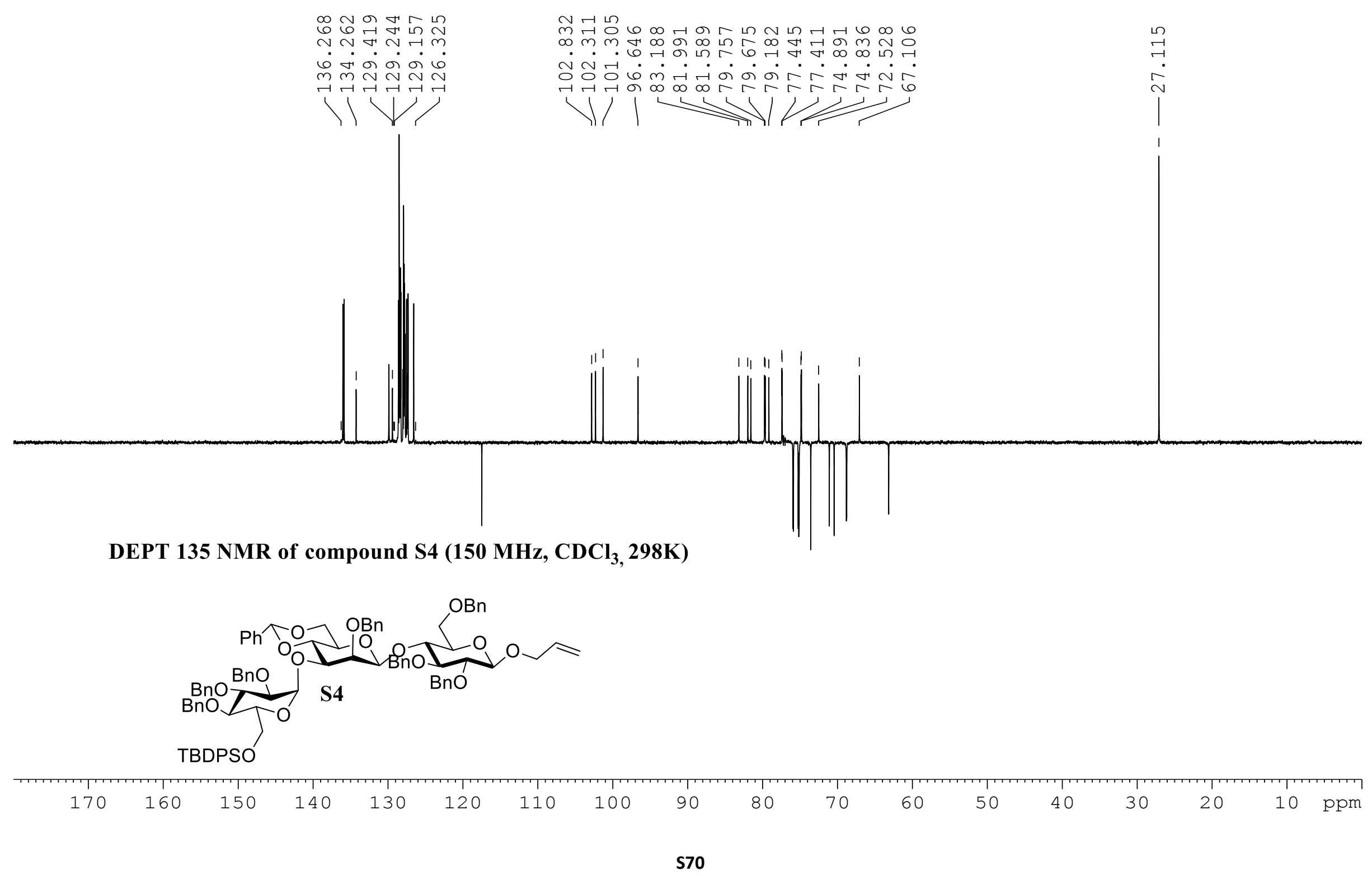


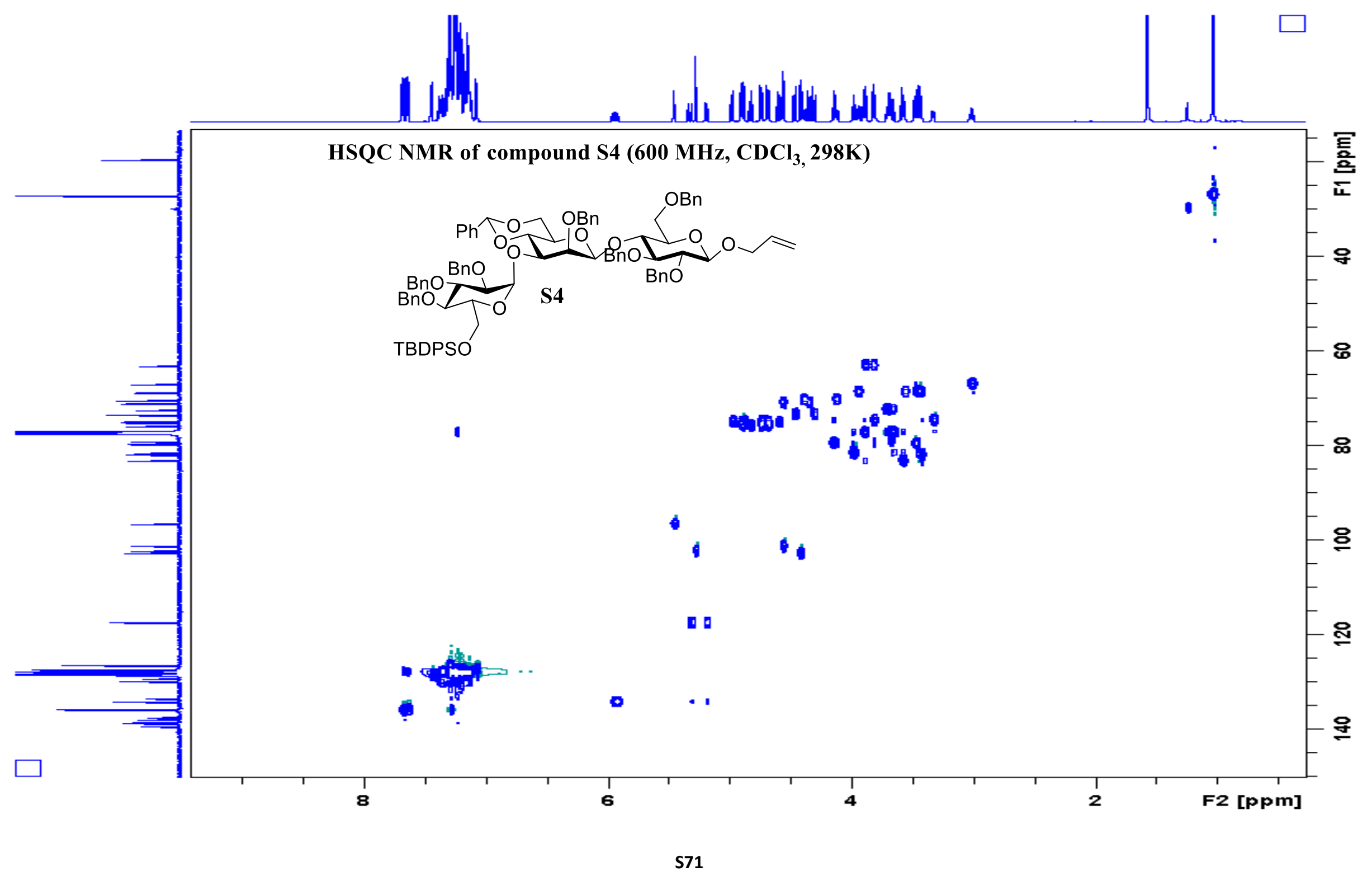




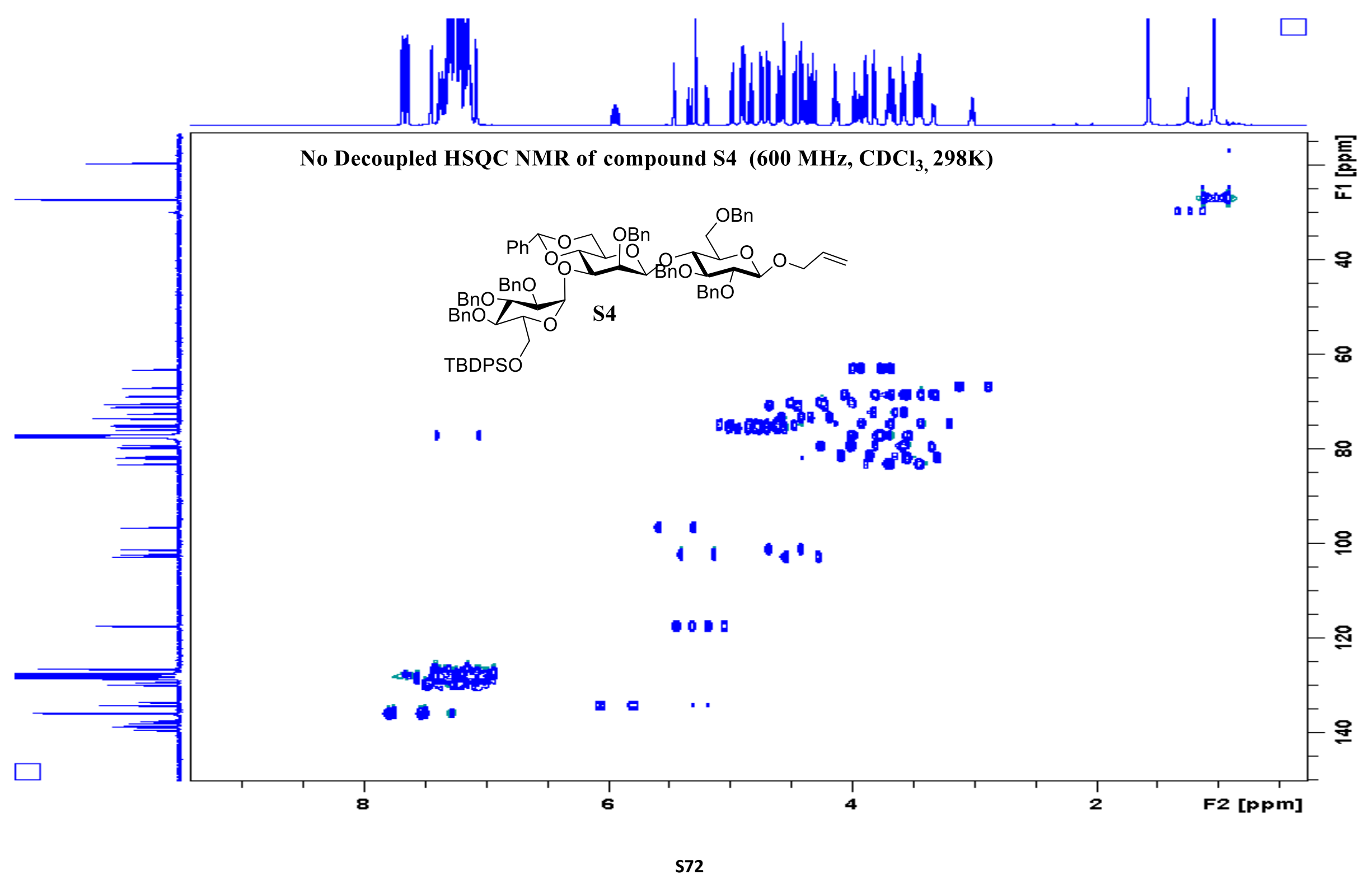




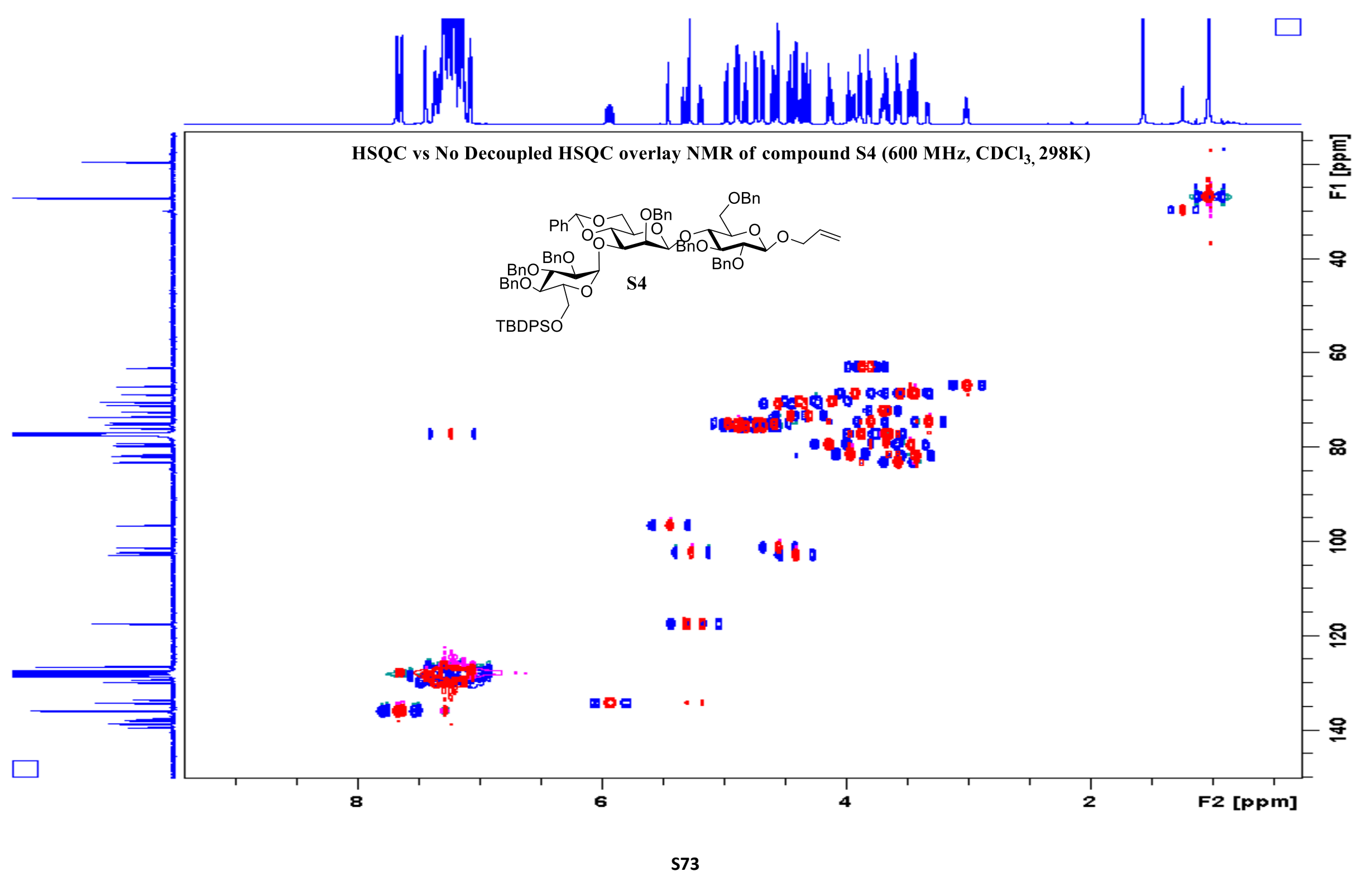




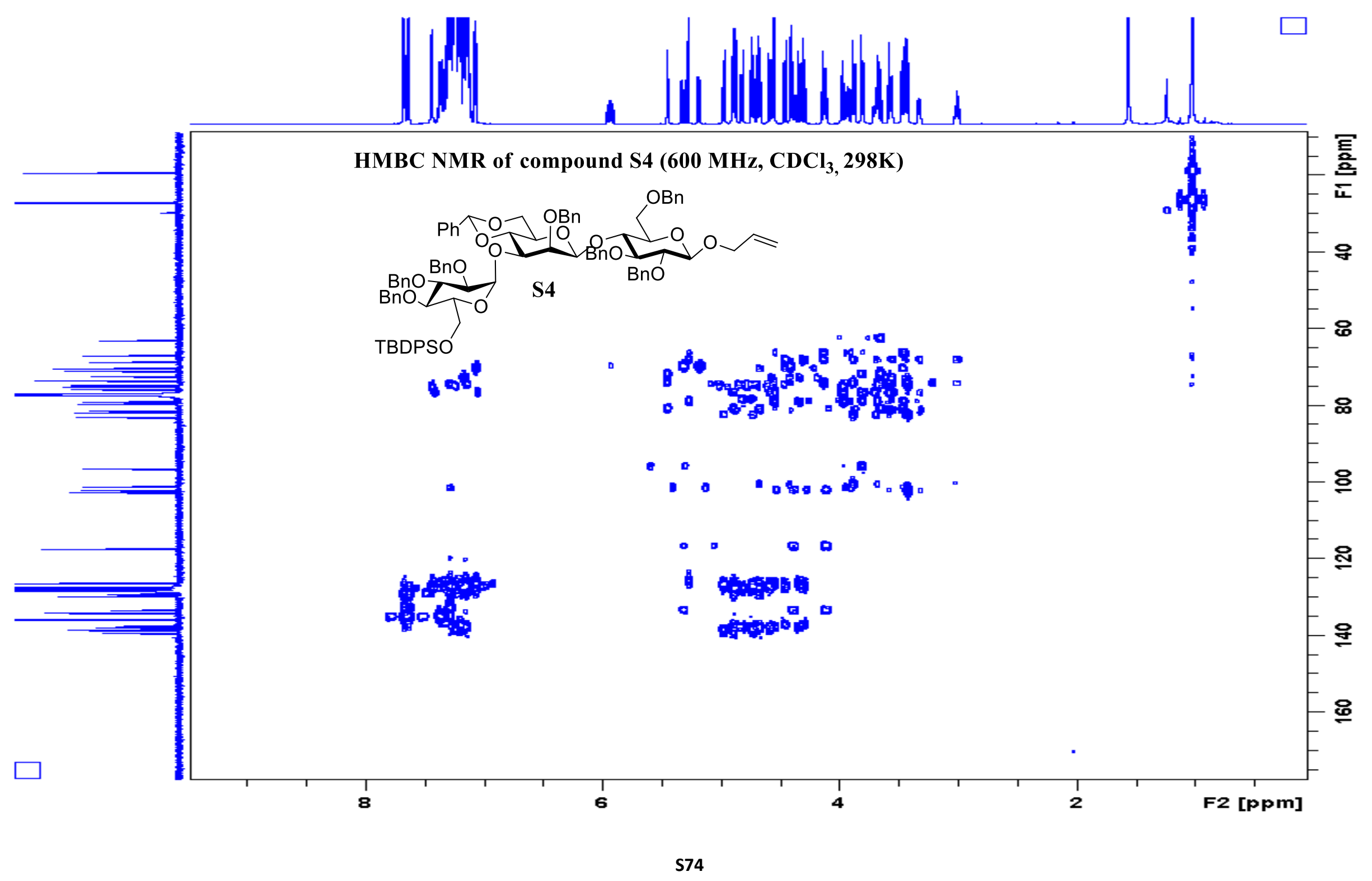




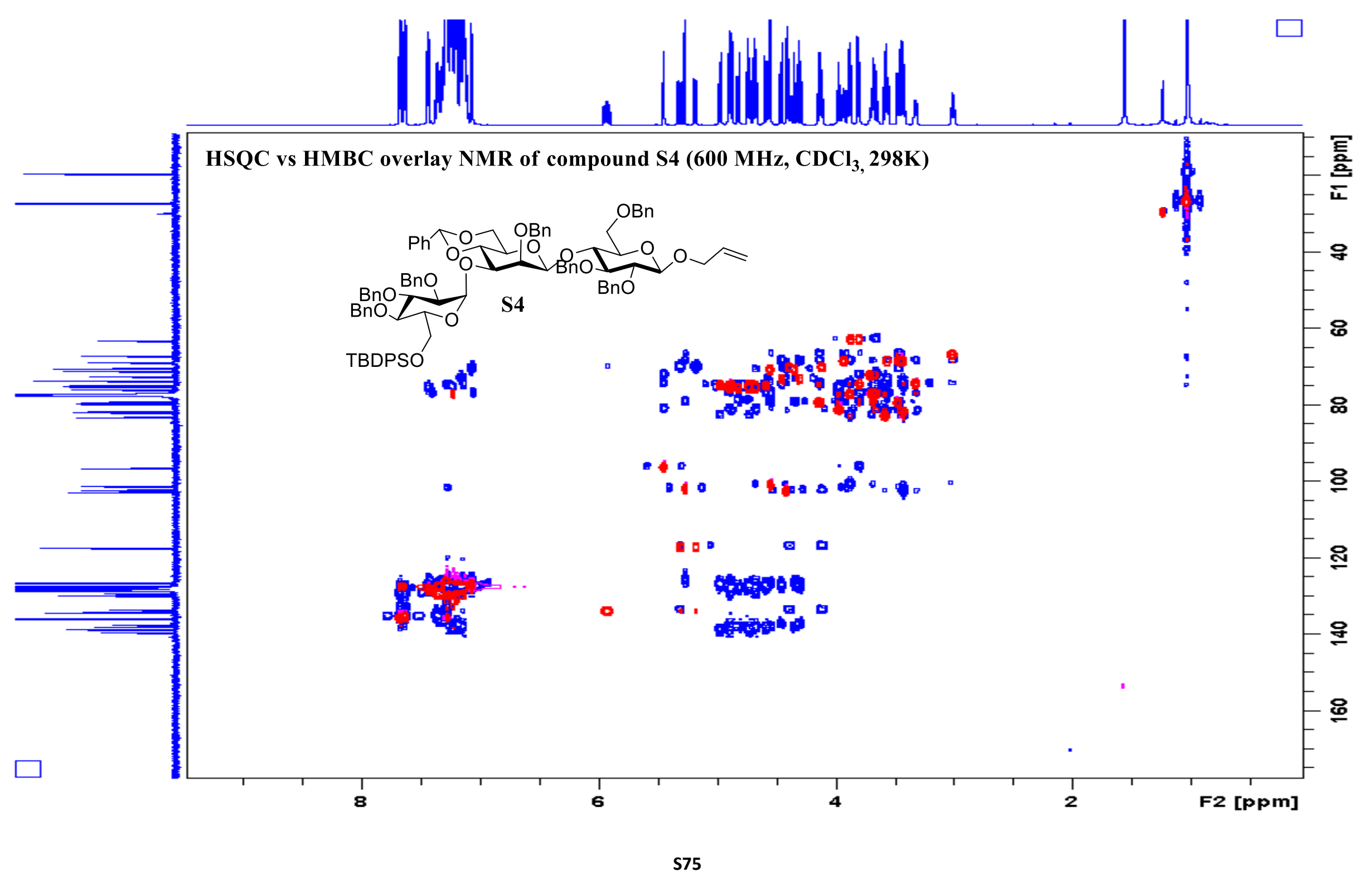




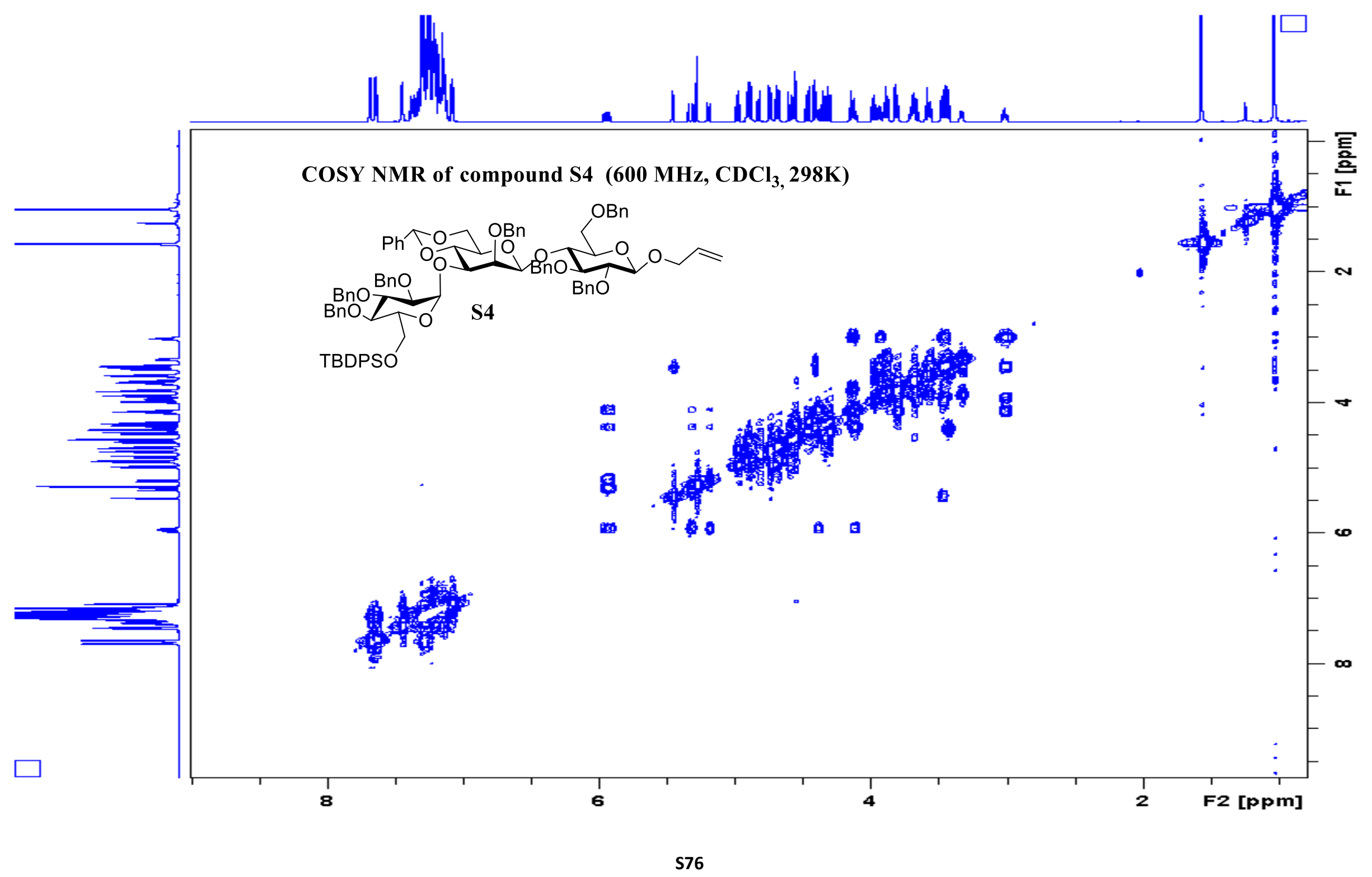




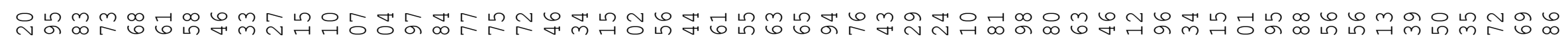

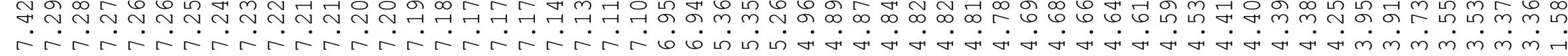

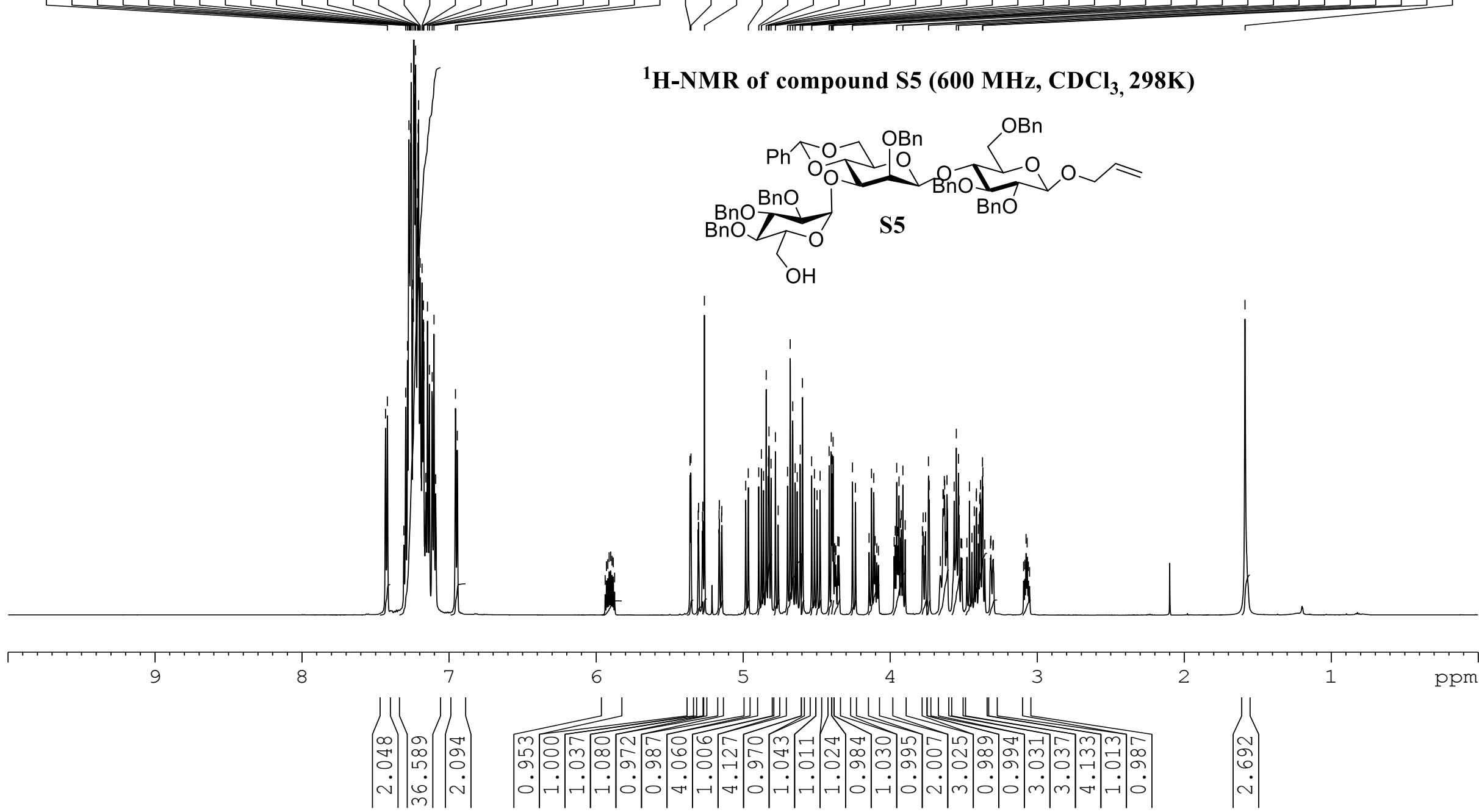




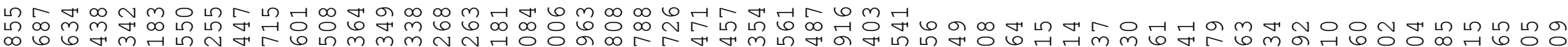

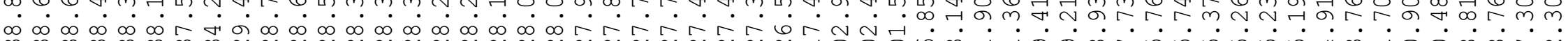

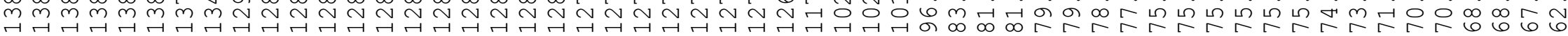

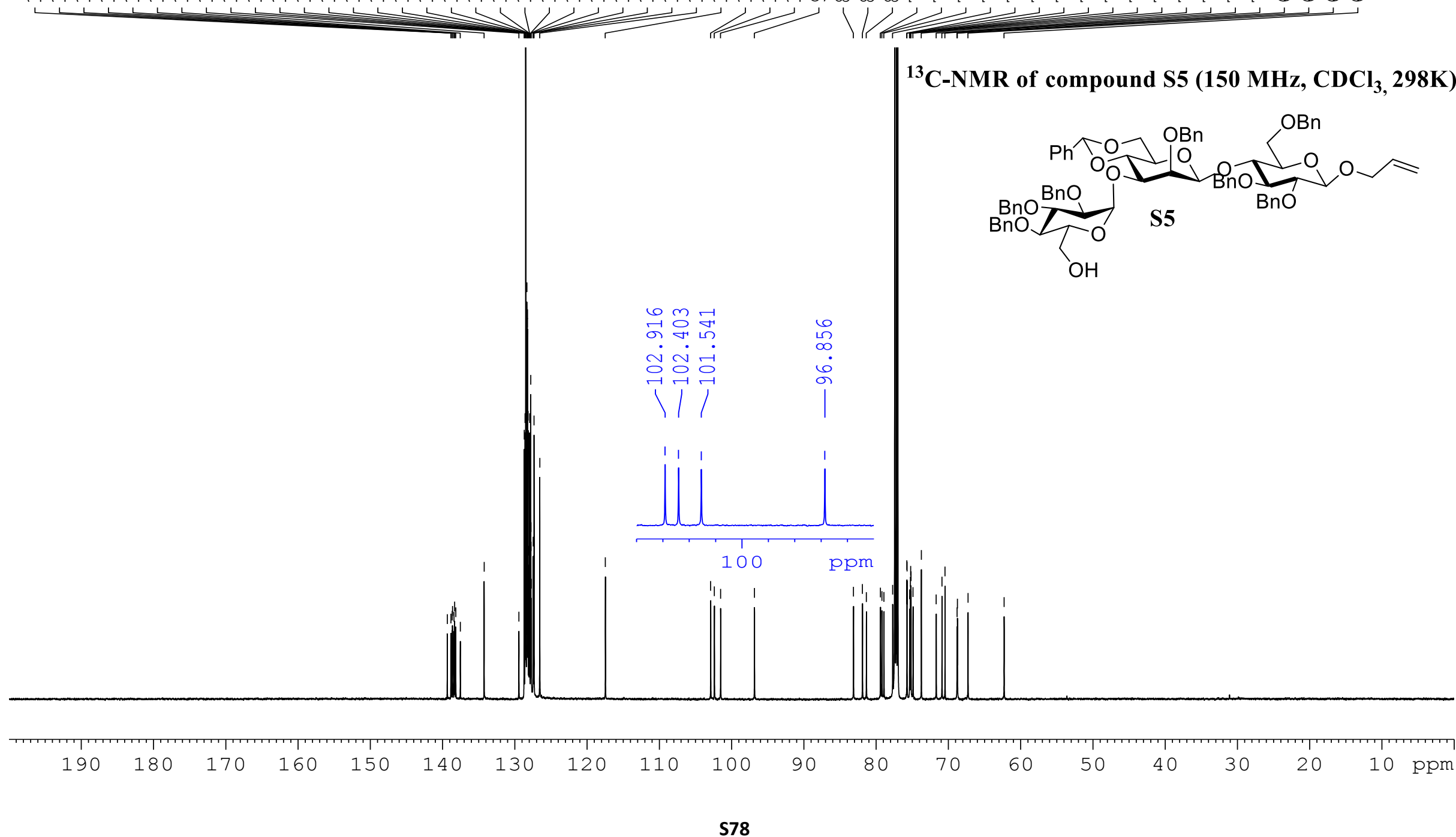




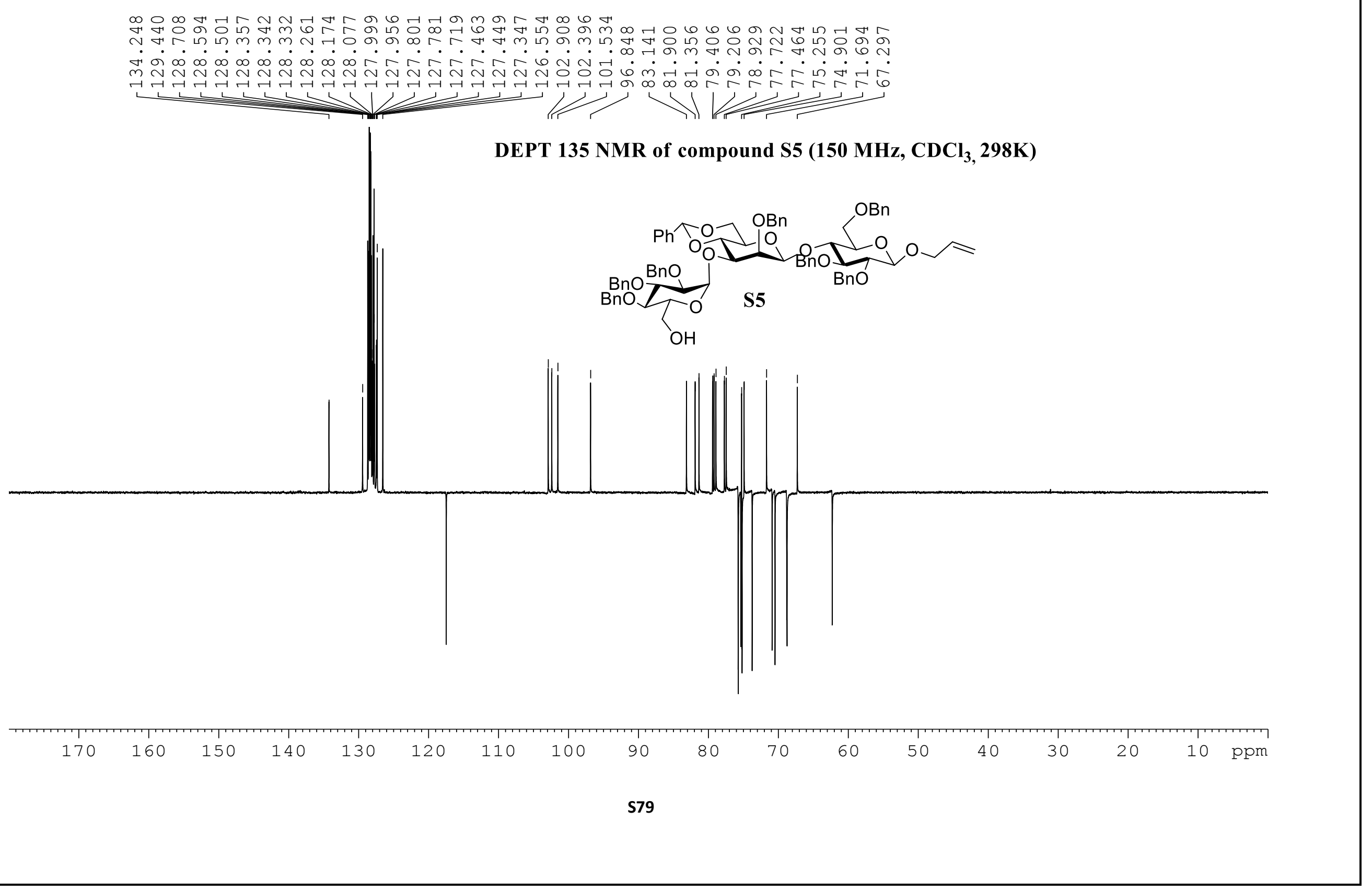




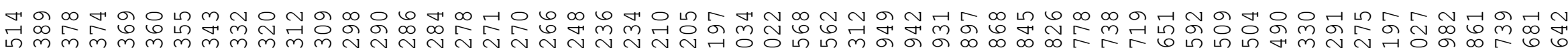

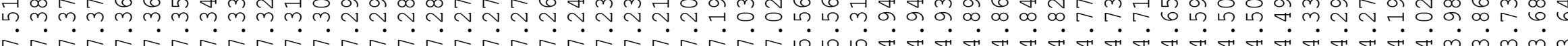

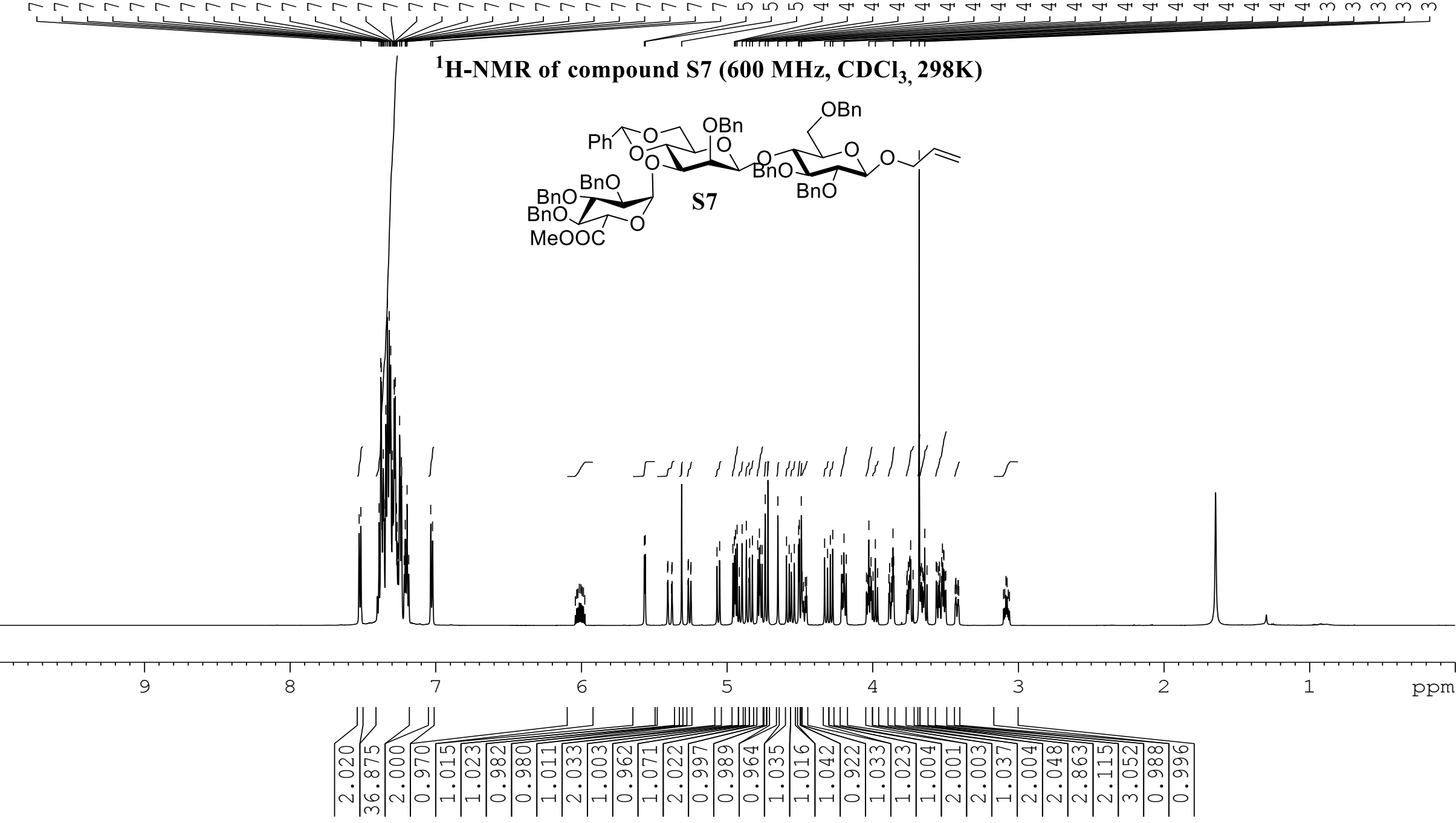




$$
\pi
$$




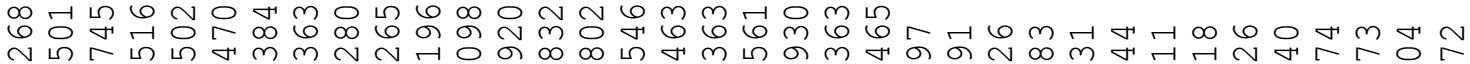

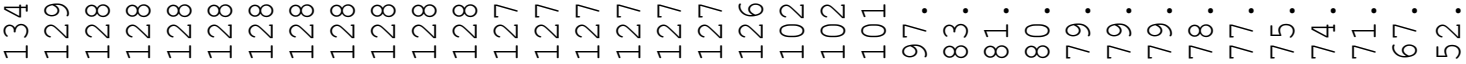

我

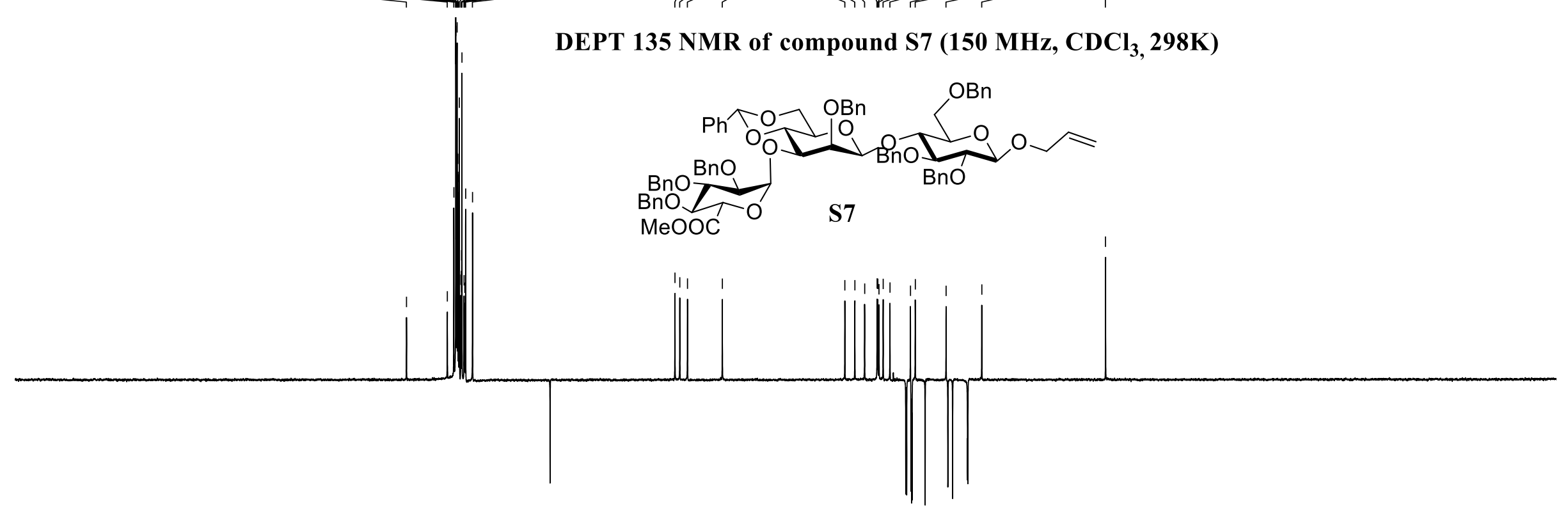

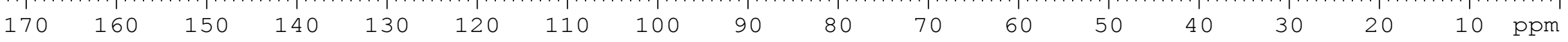




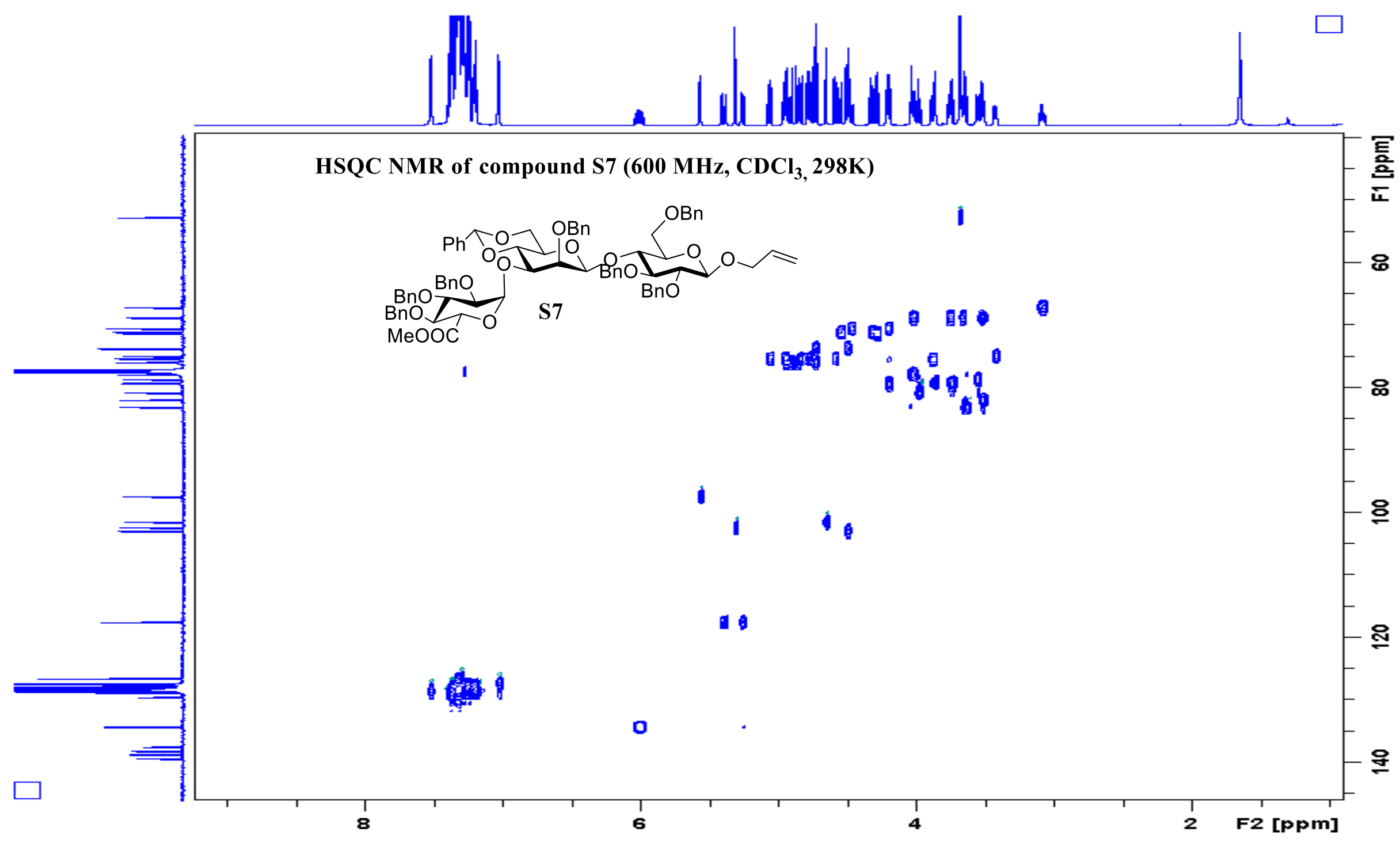

583 


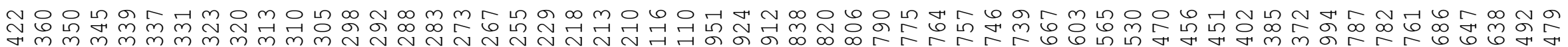

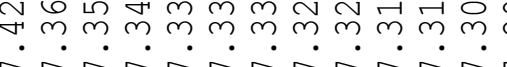

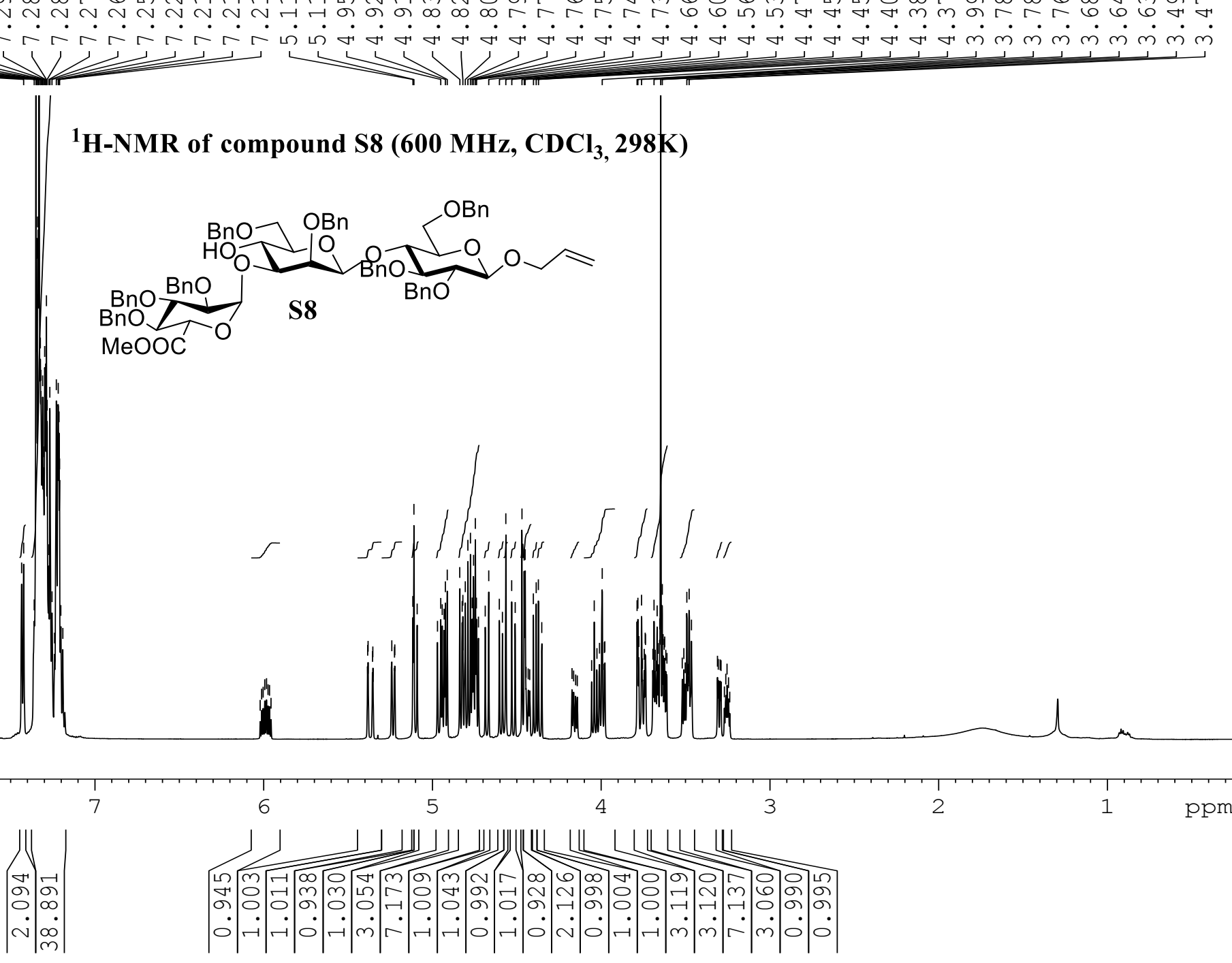




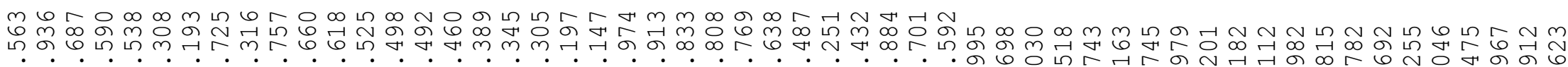

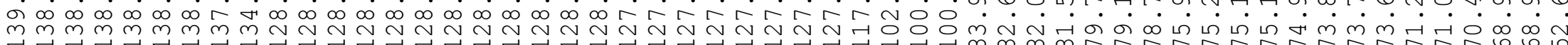

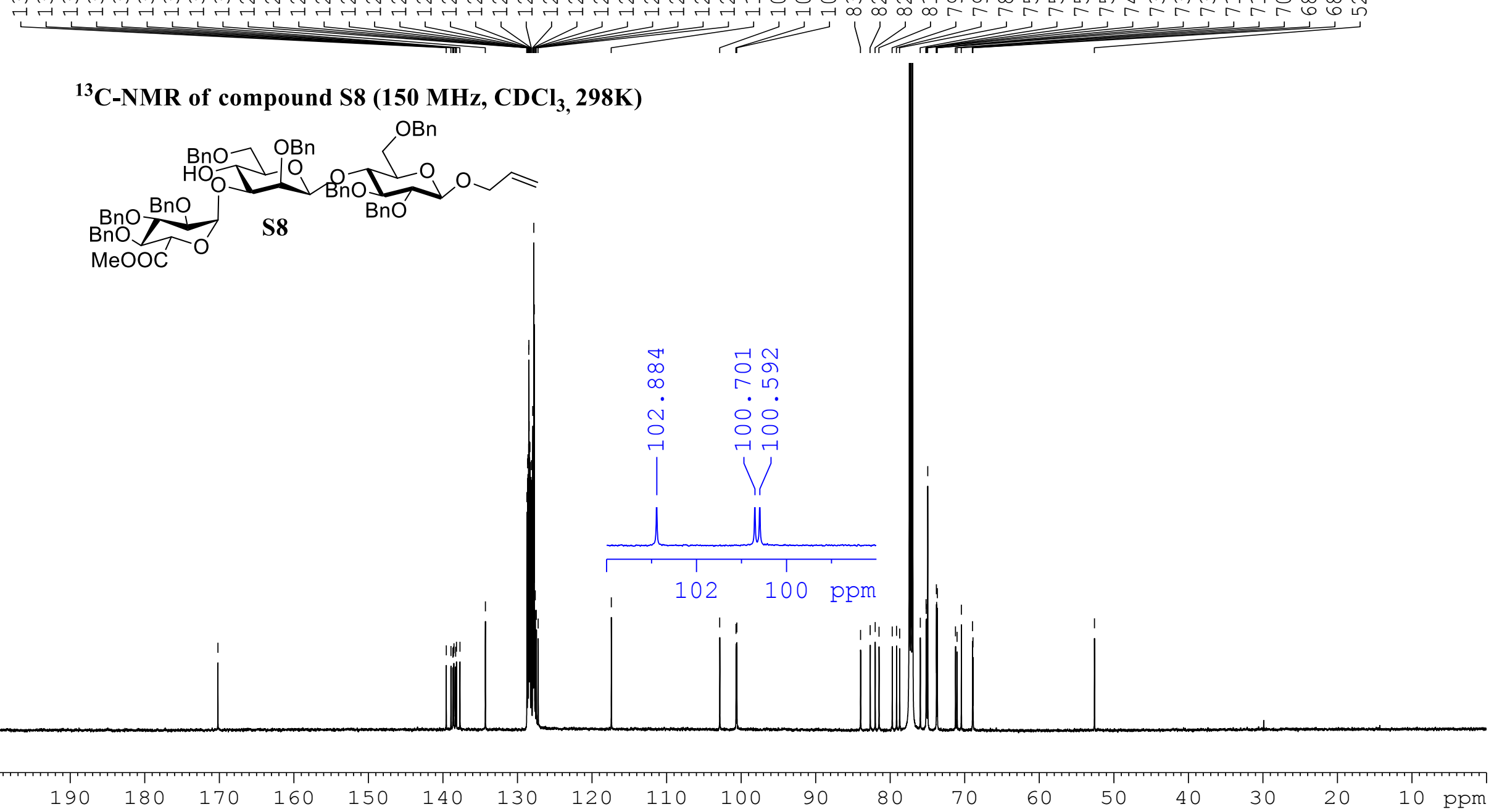



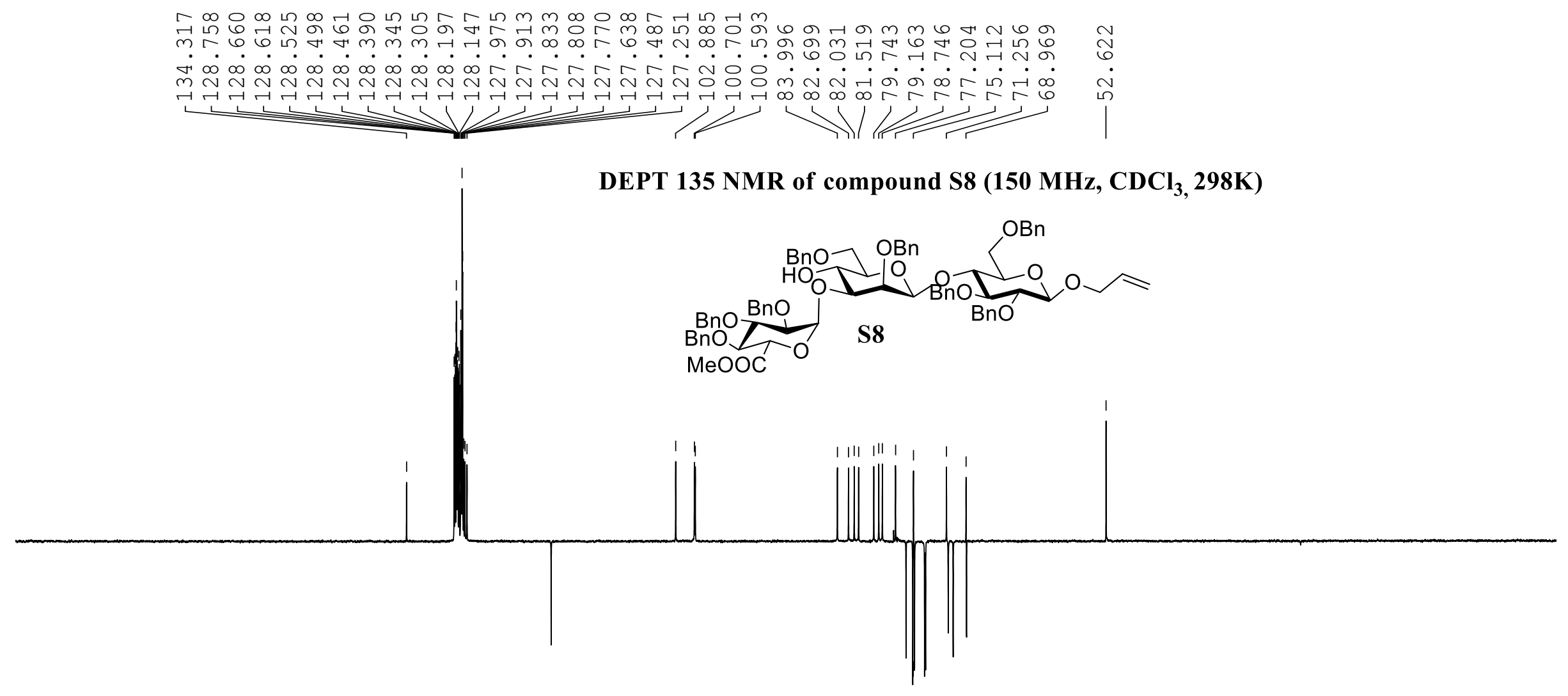

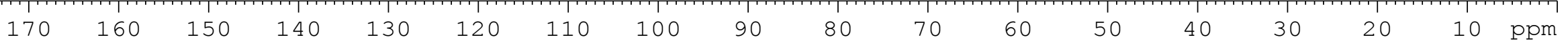




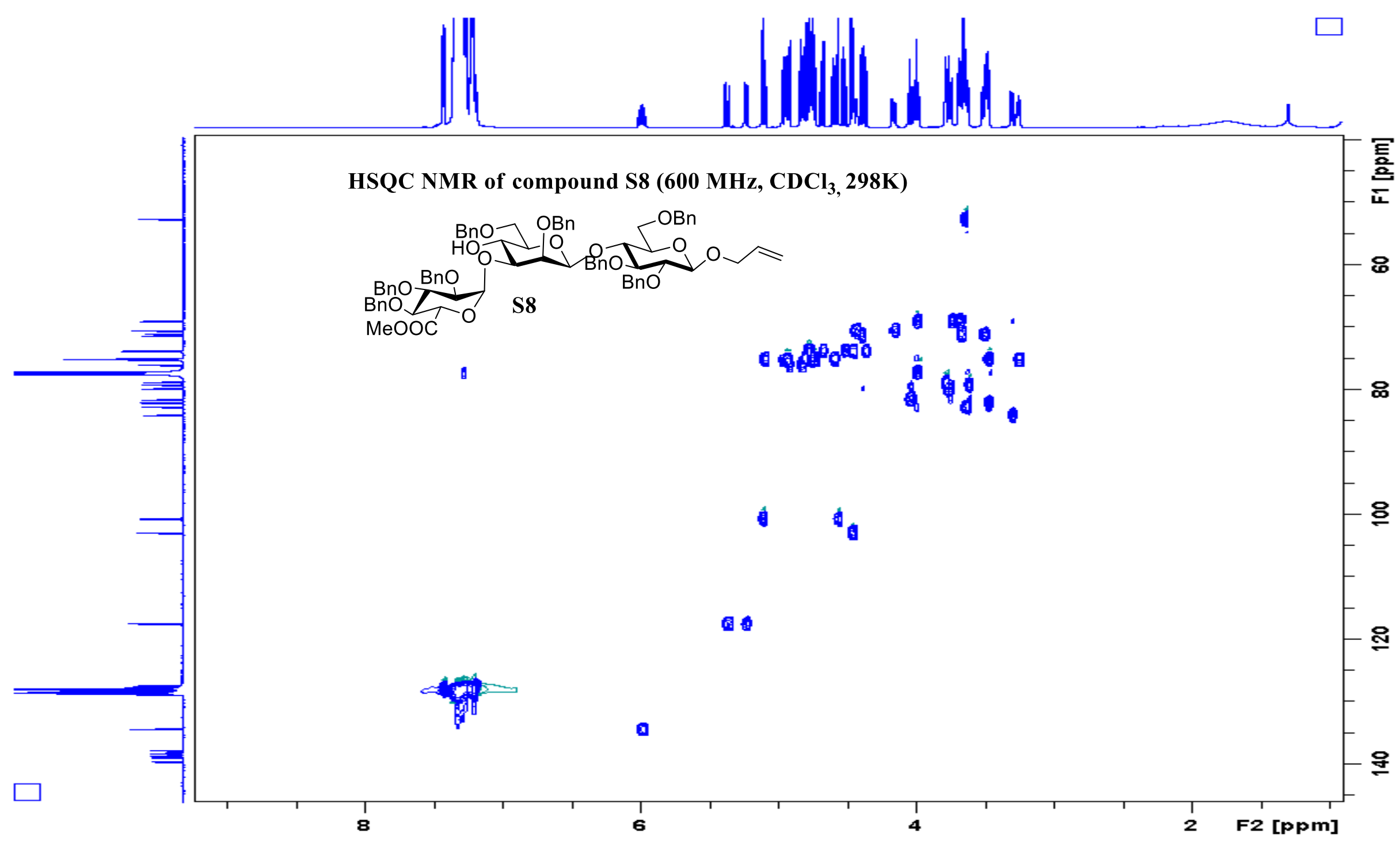

S87 


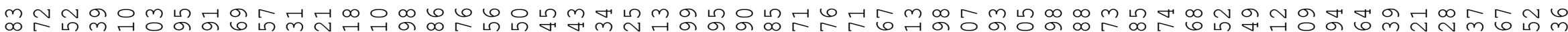
o

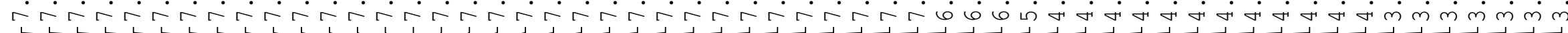

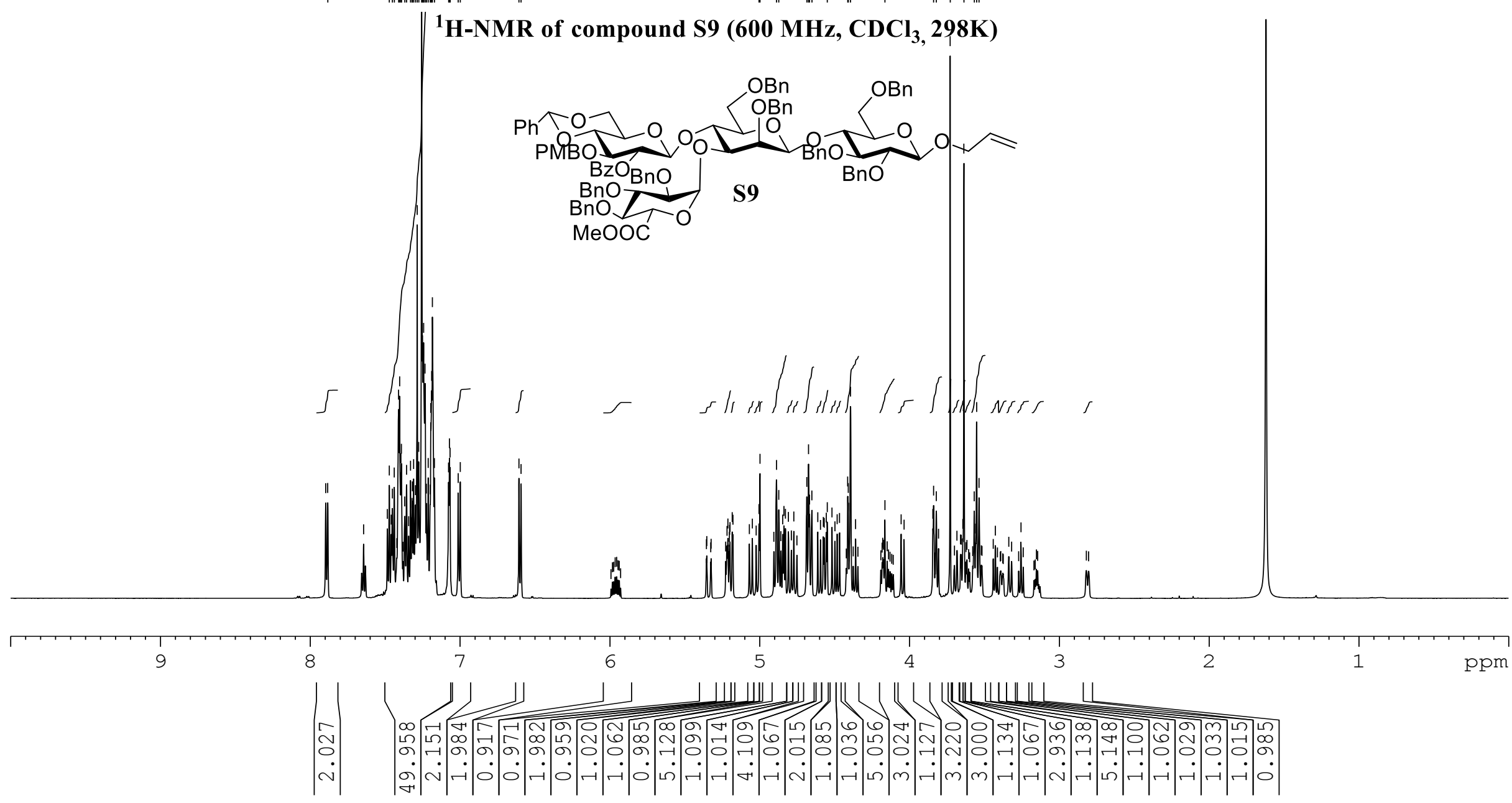




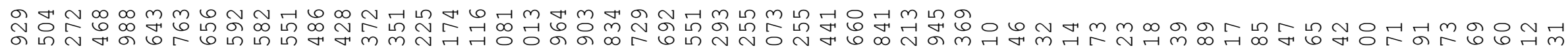
ळ

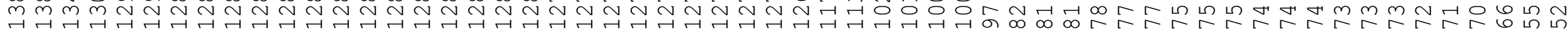

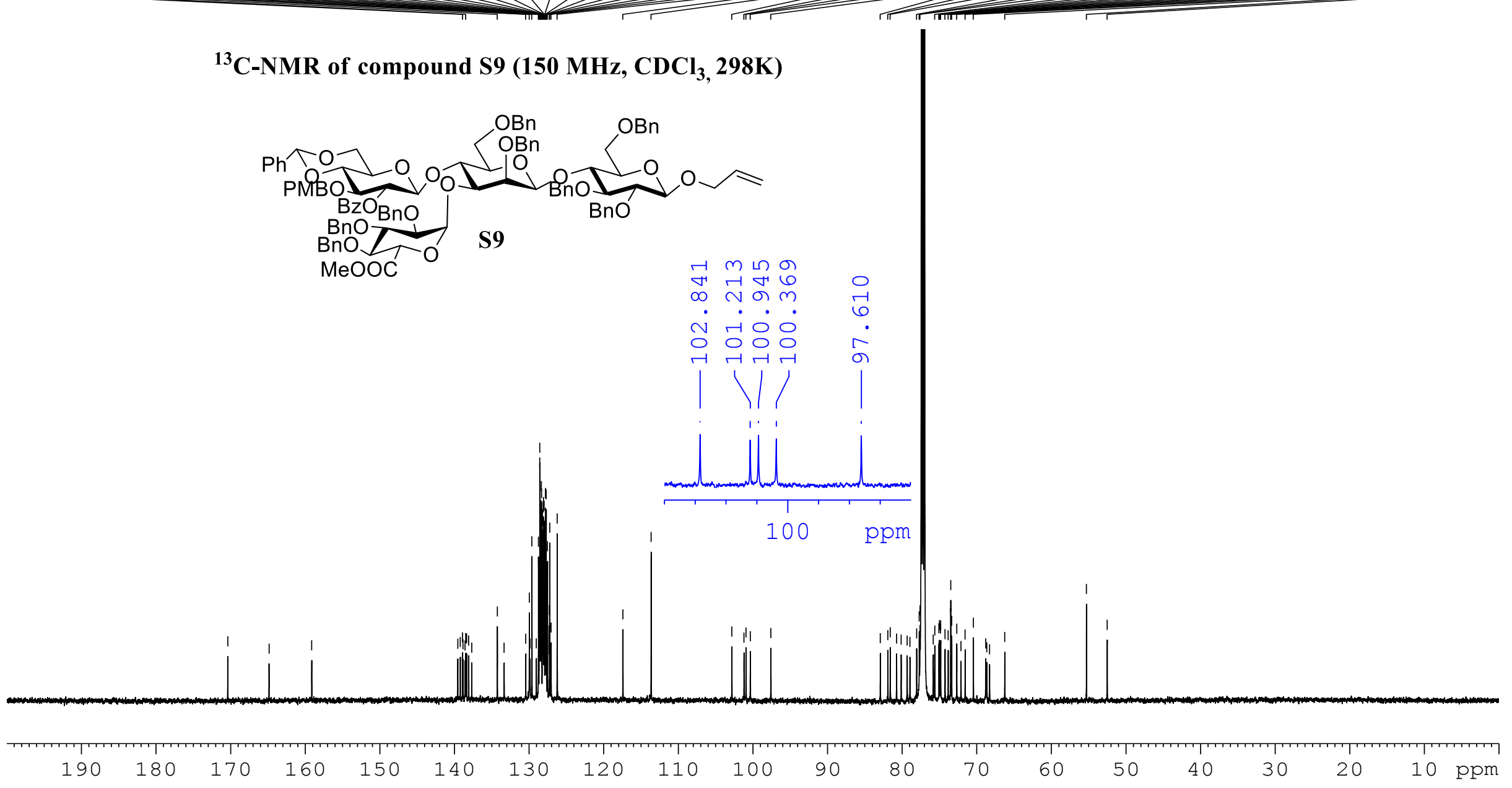




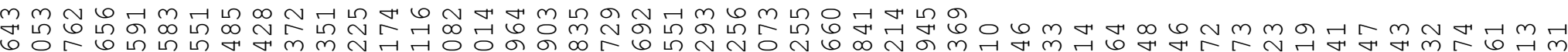

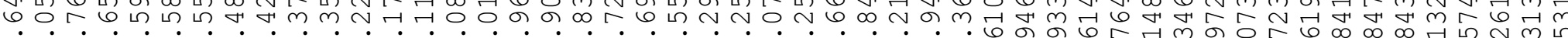

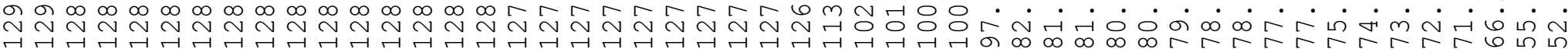

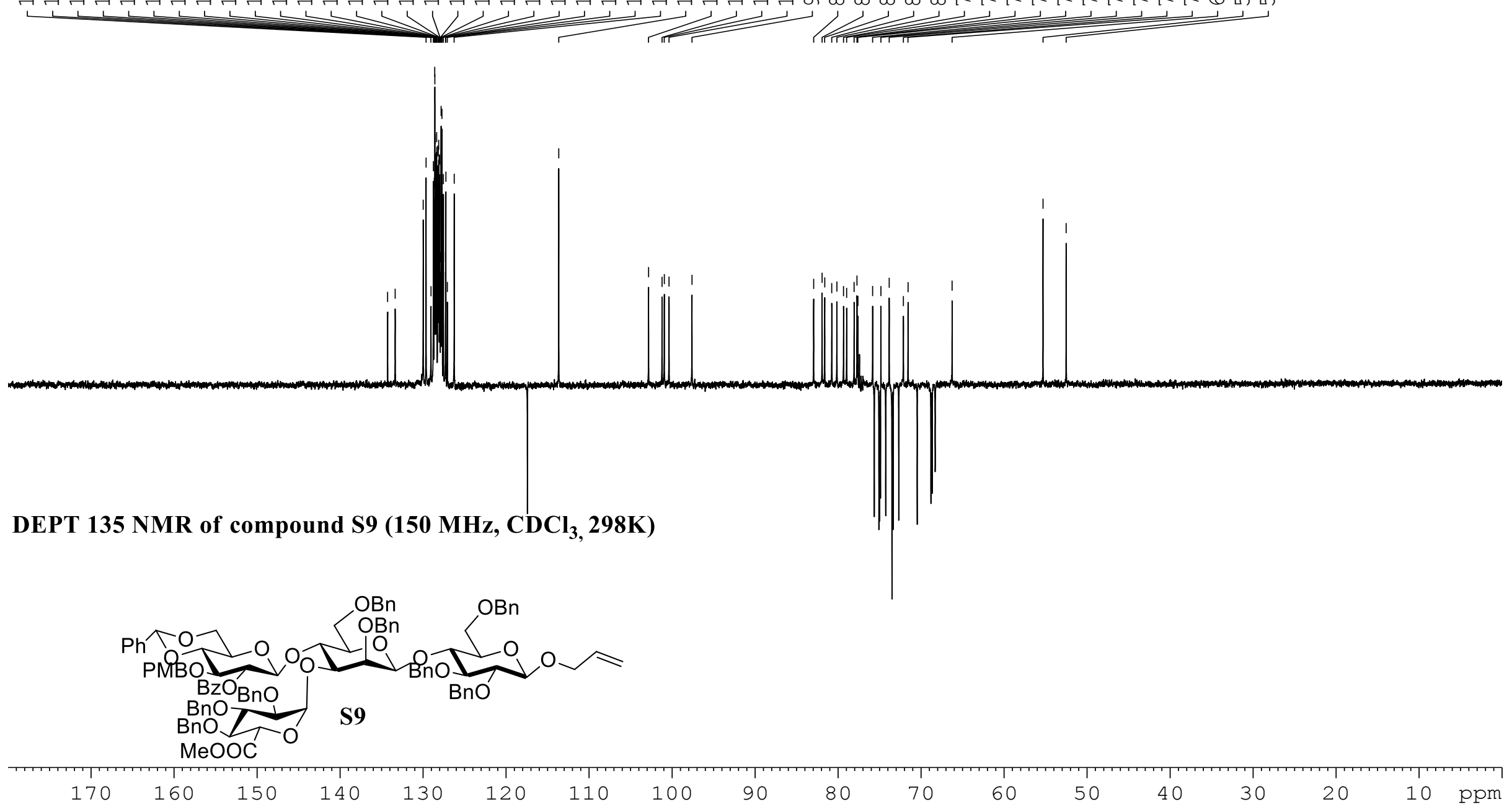




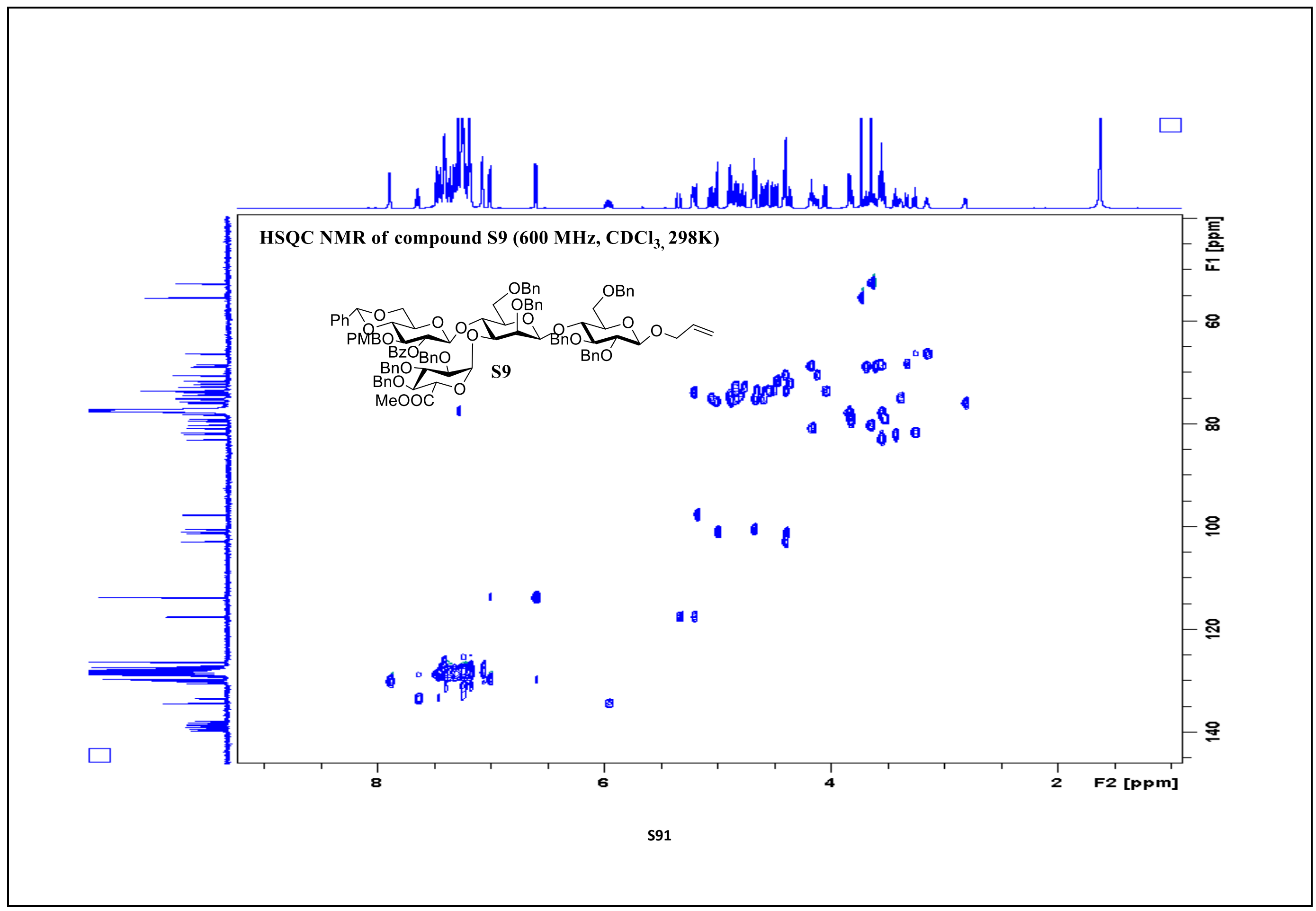




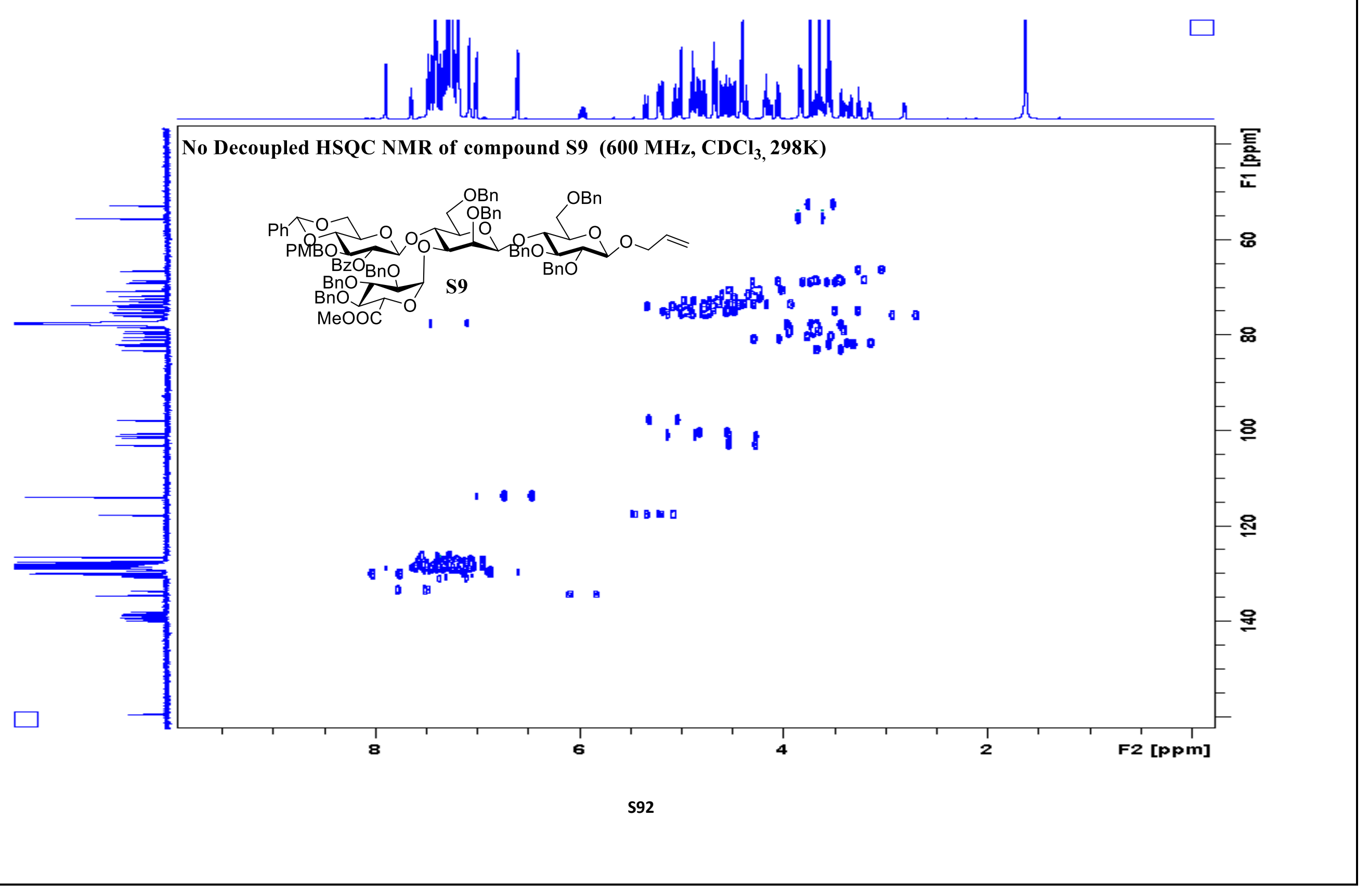




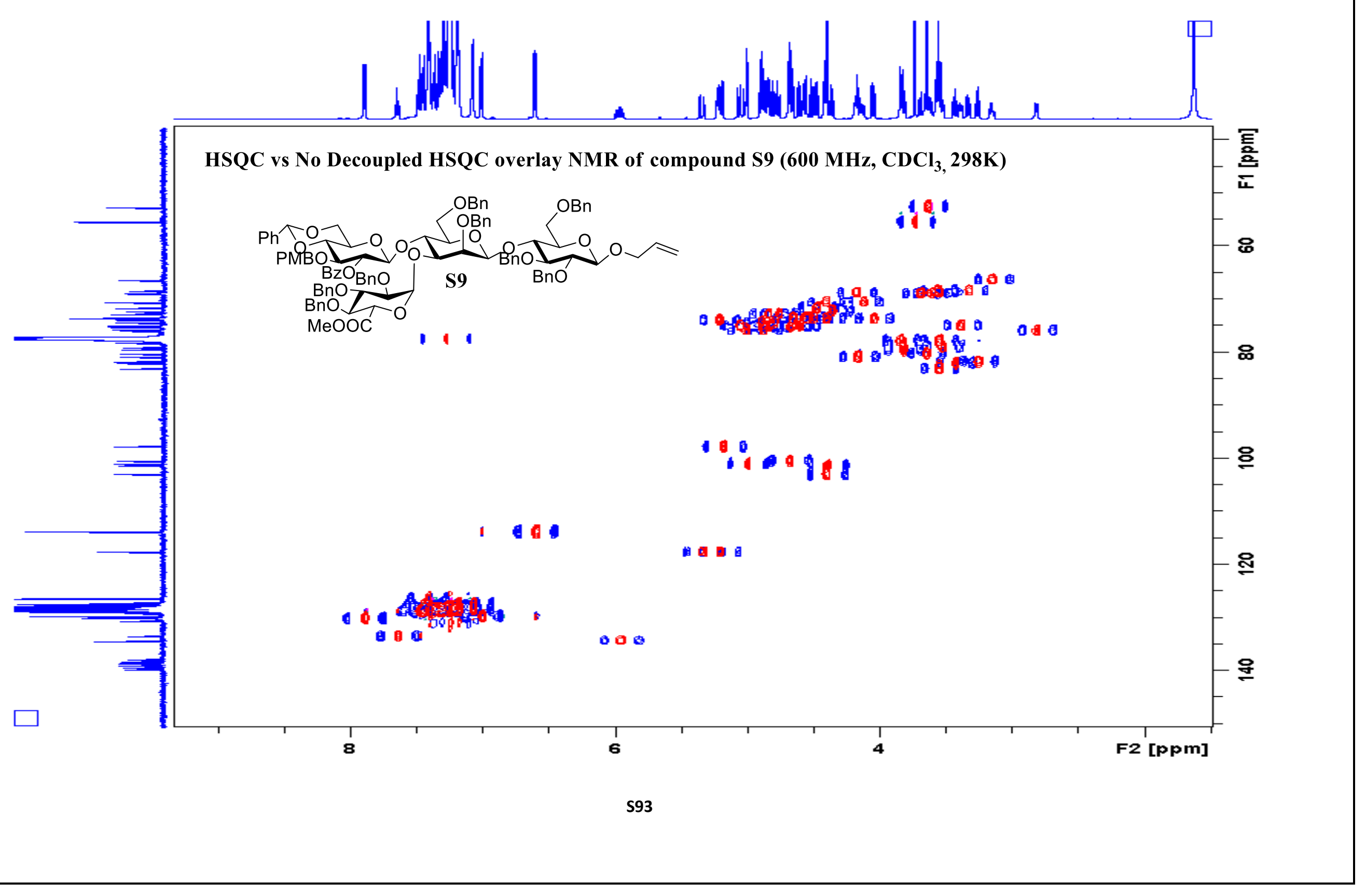




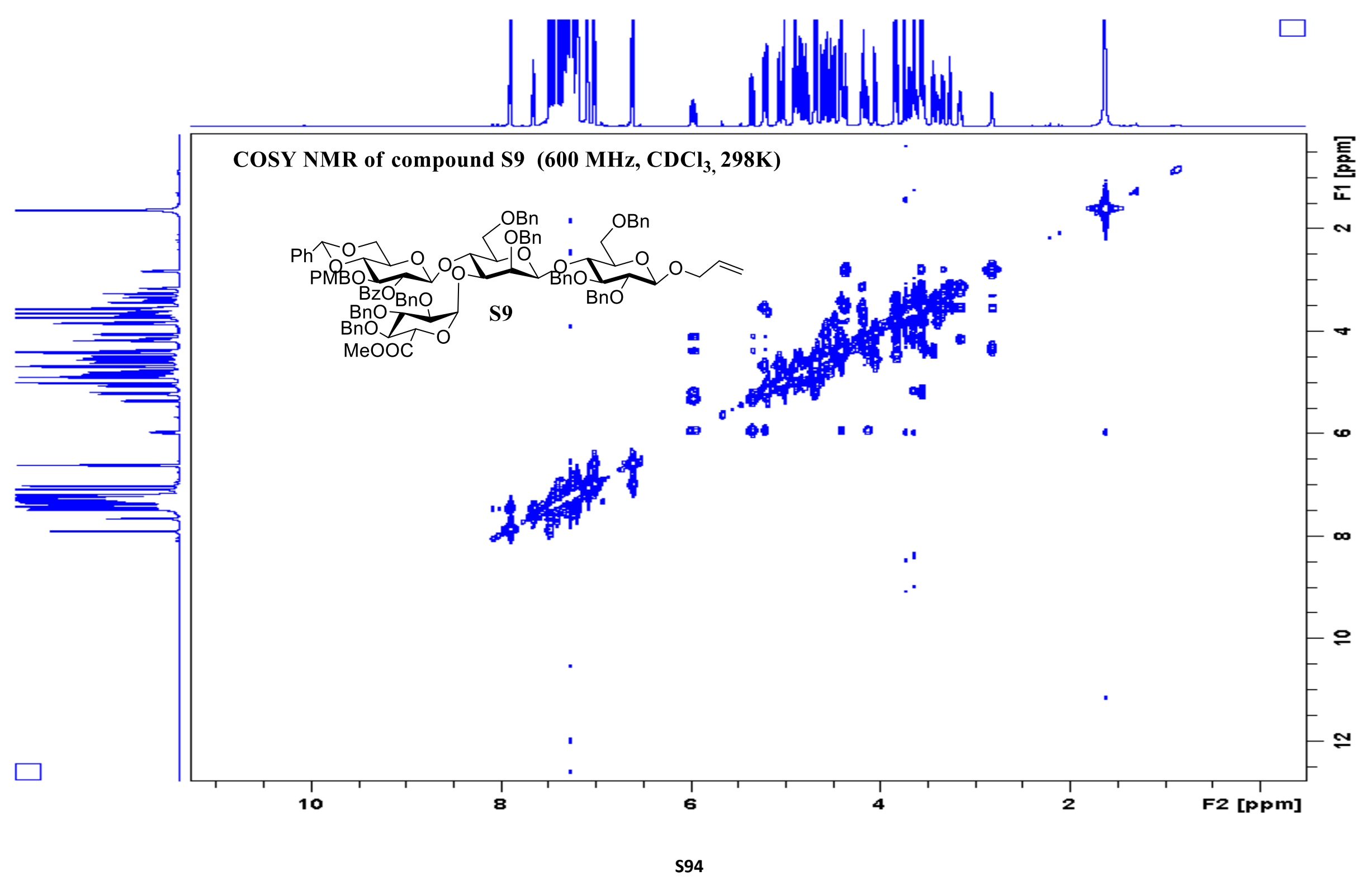




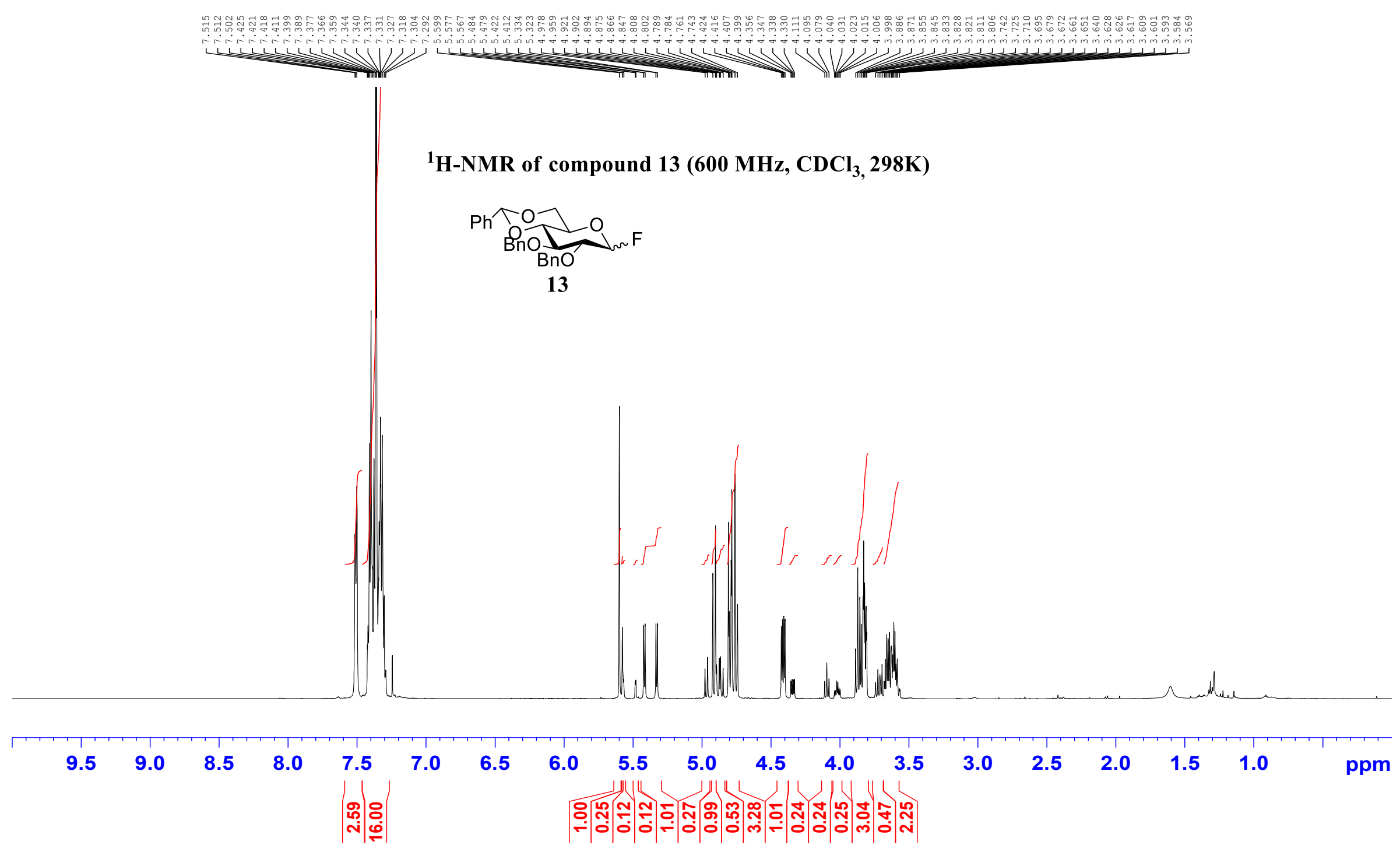

S95 


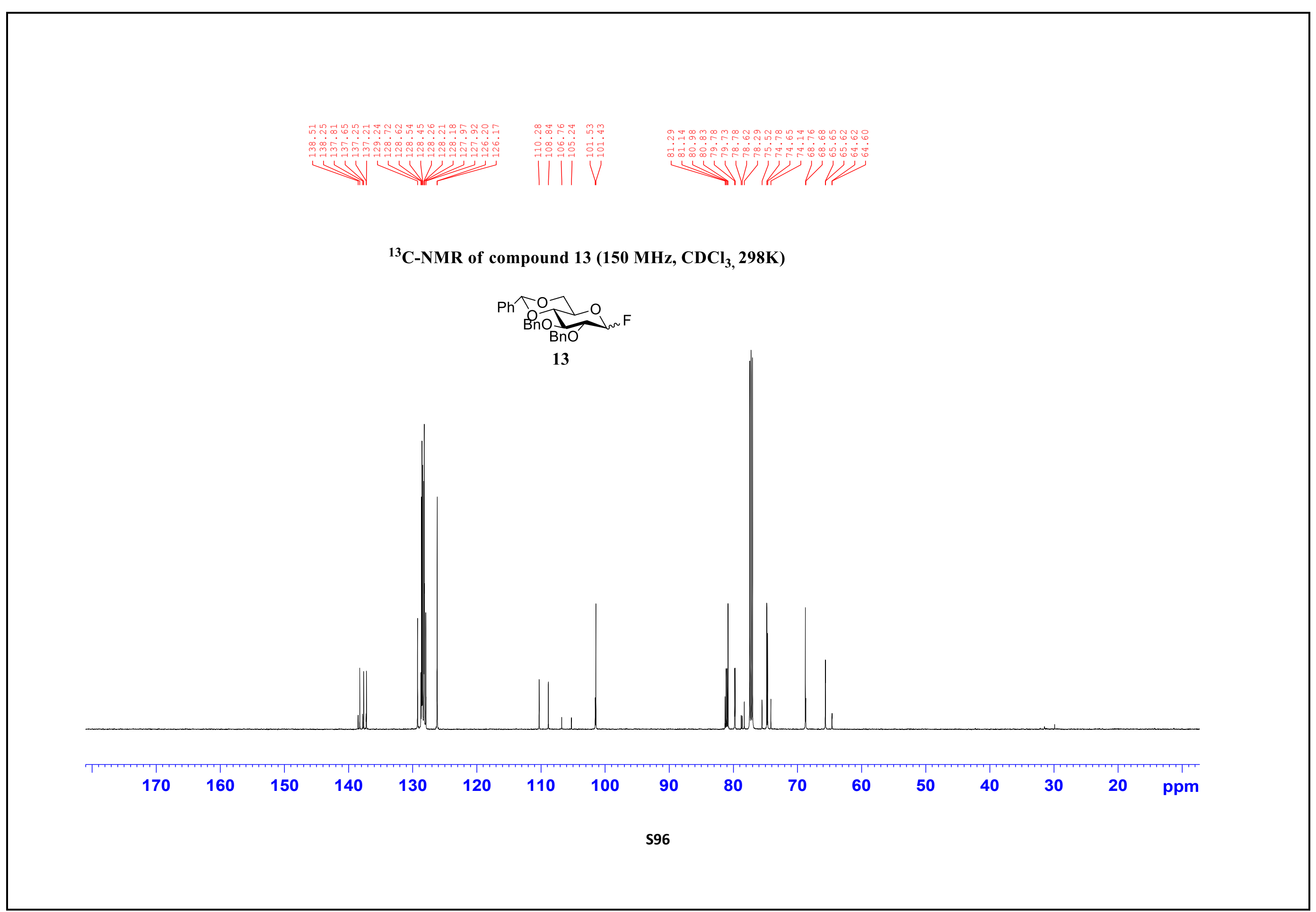




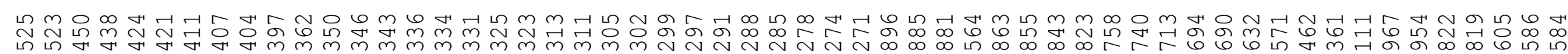

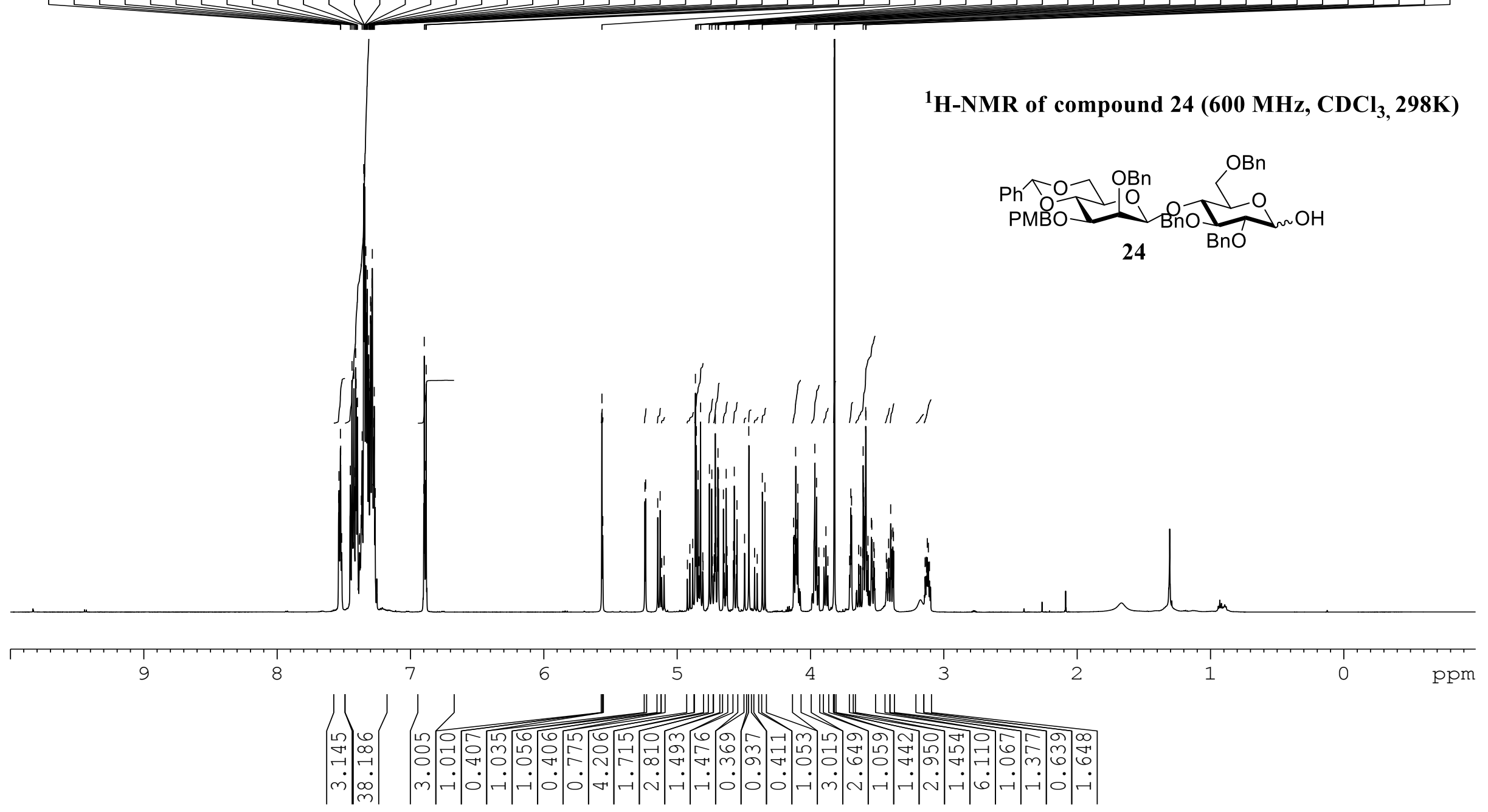


馬贷

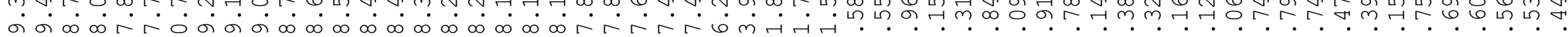

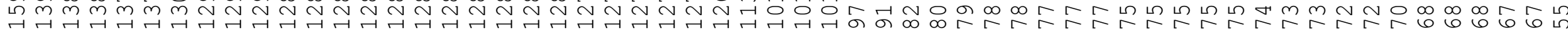

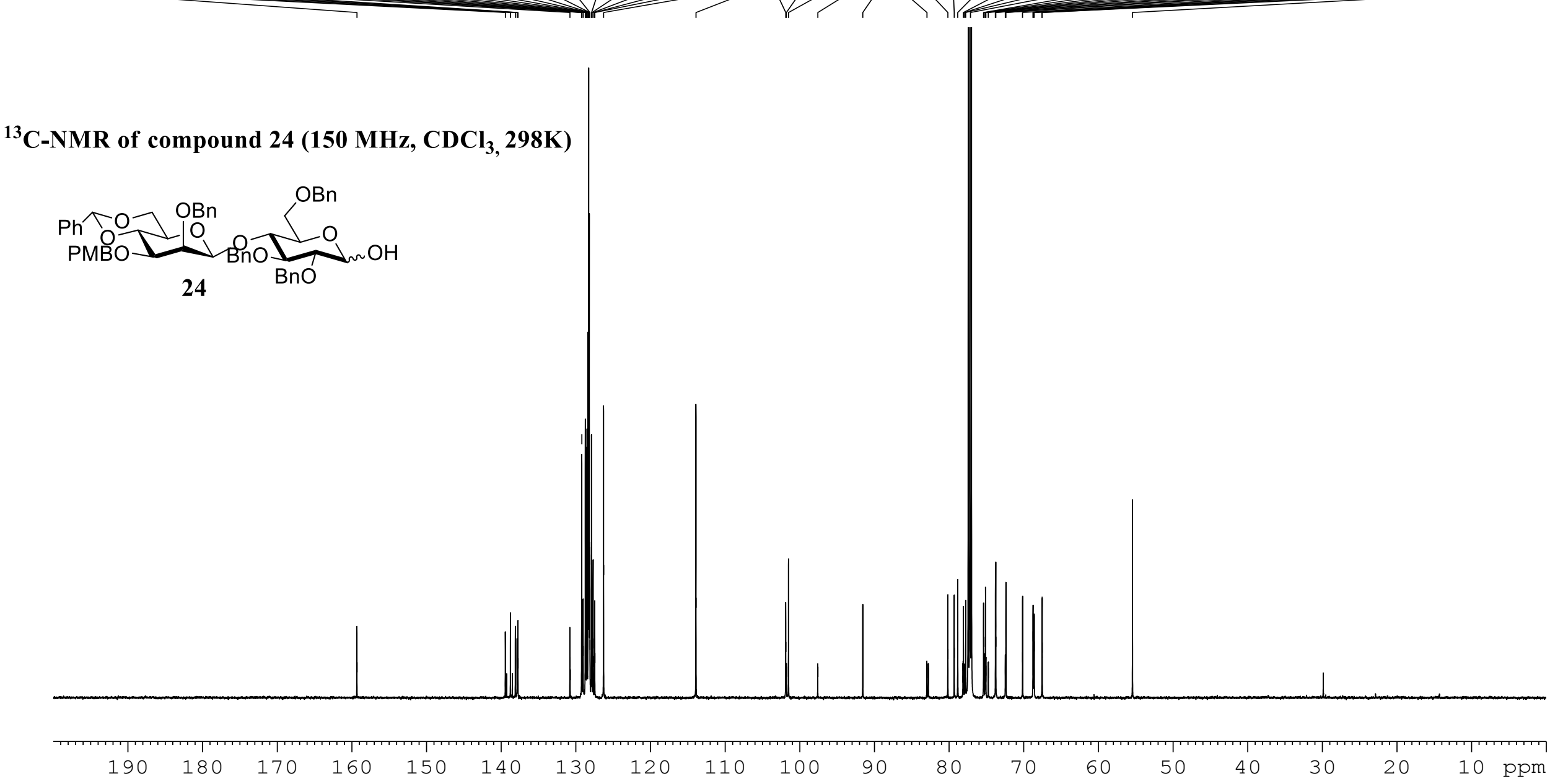




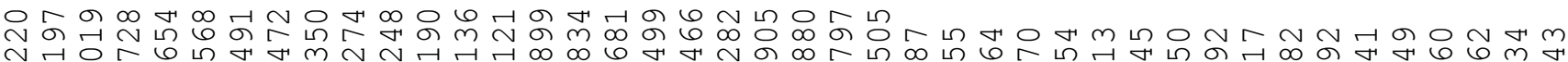

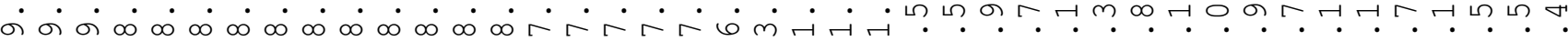

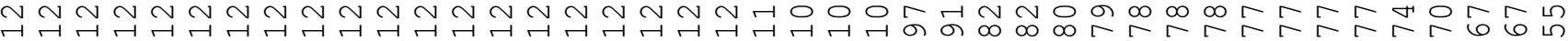

年

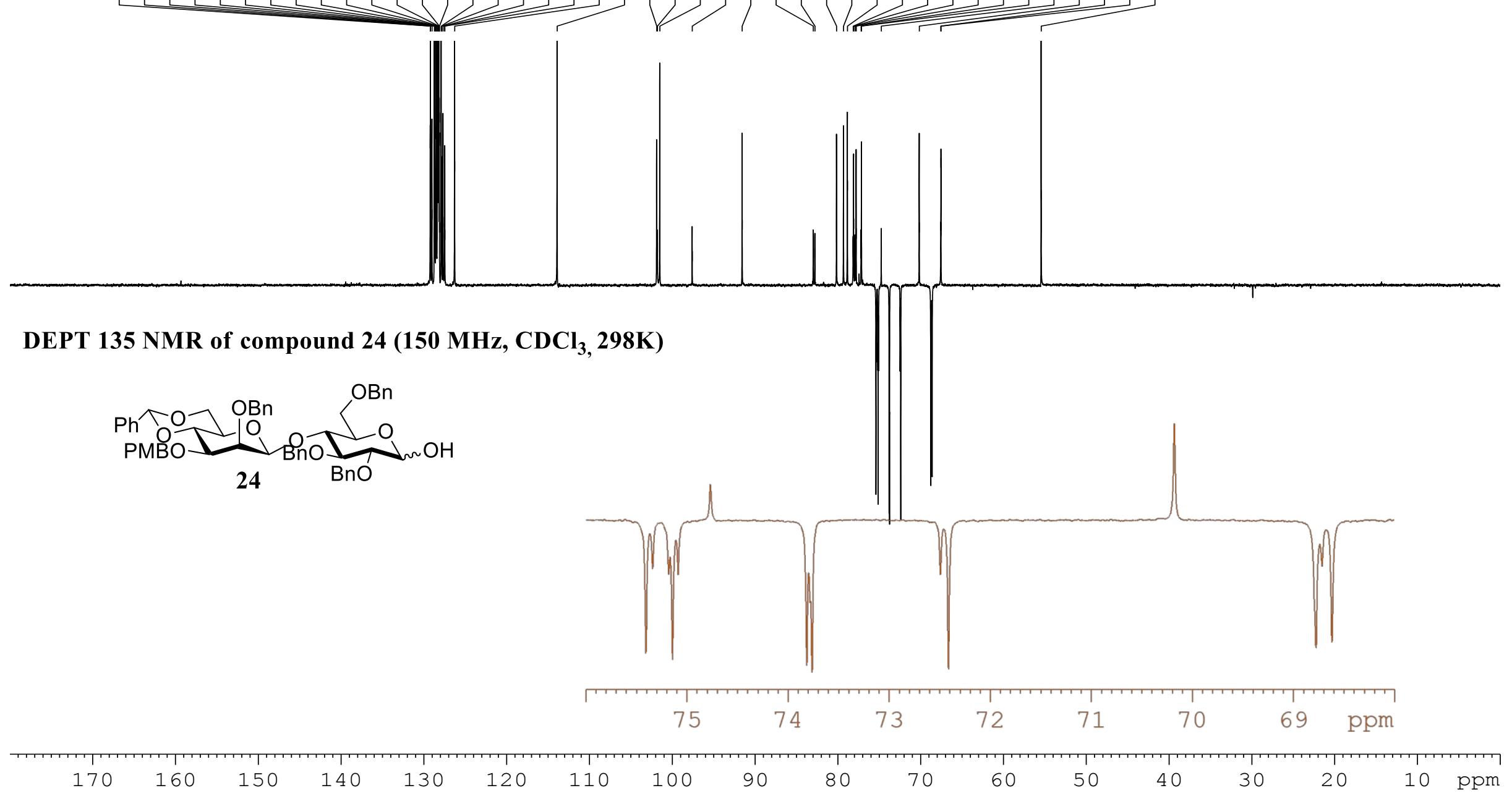




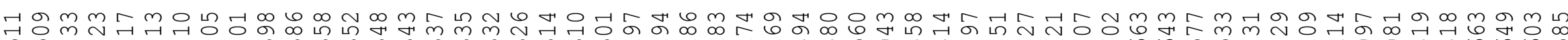

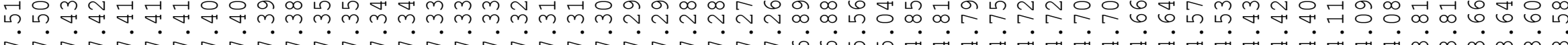

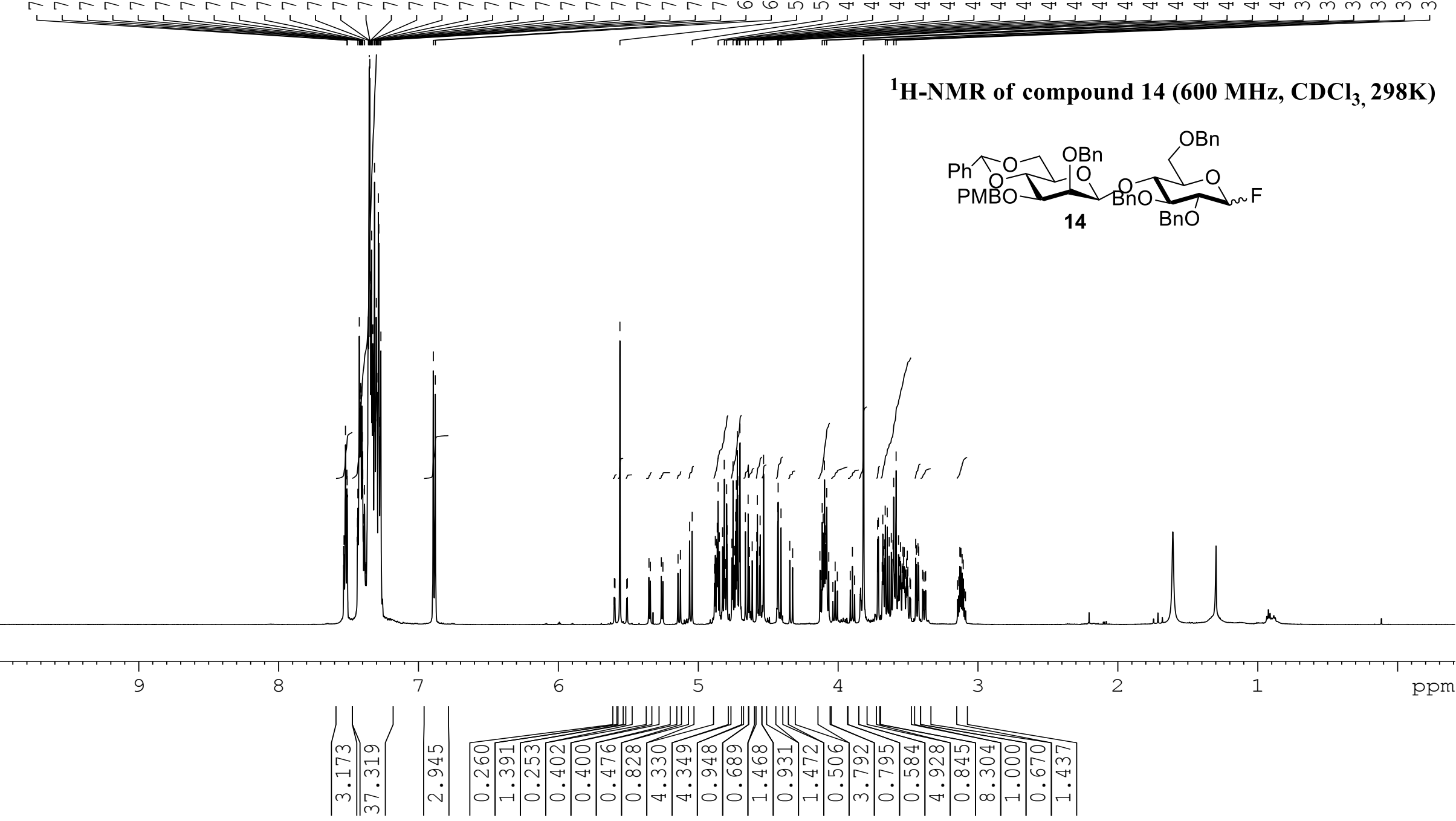




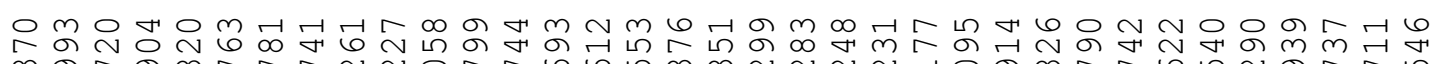

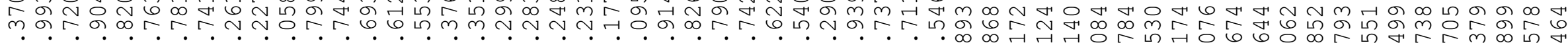

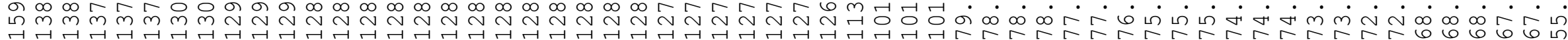

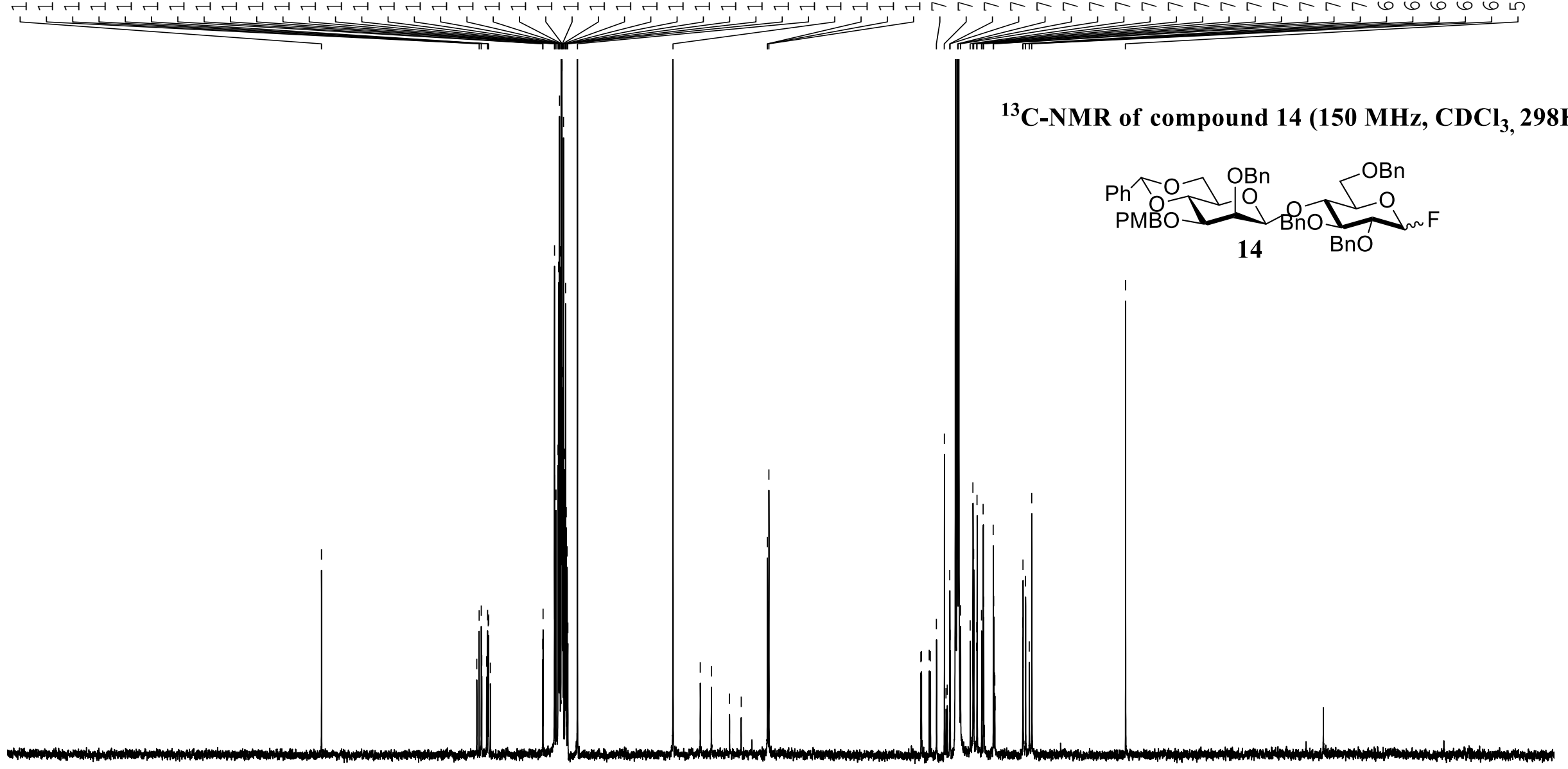

190

180

170

160

150

40

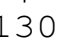

120

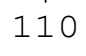

100

90

80

70

60

50

40

30

2010 ppm

S101 

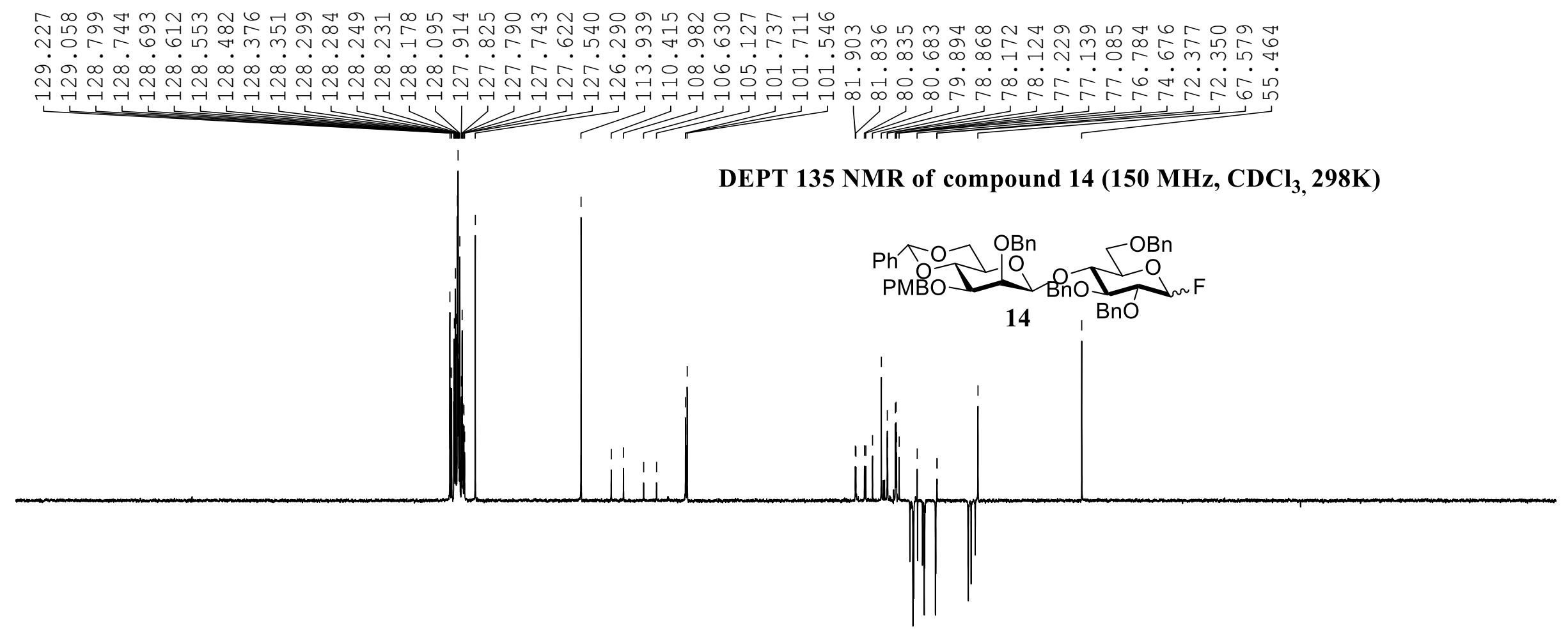

$\begin{array}{llllllllllllllllllll}170 & 160 & 150 & 140 & 130 & 120 & 110 & 100 & 90 & 80 & 70 & 60 & 50 & 40 & 30 & 20 & 10 & \text { ppm }\end{array}$




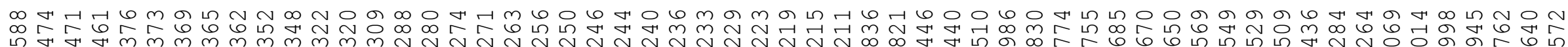

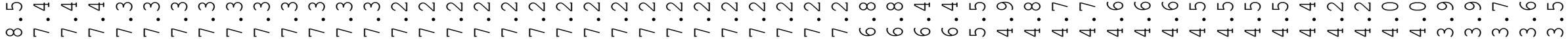

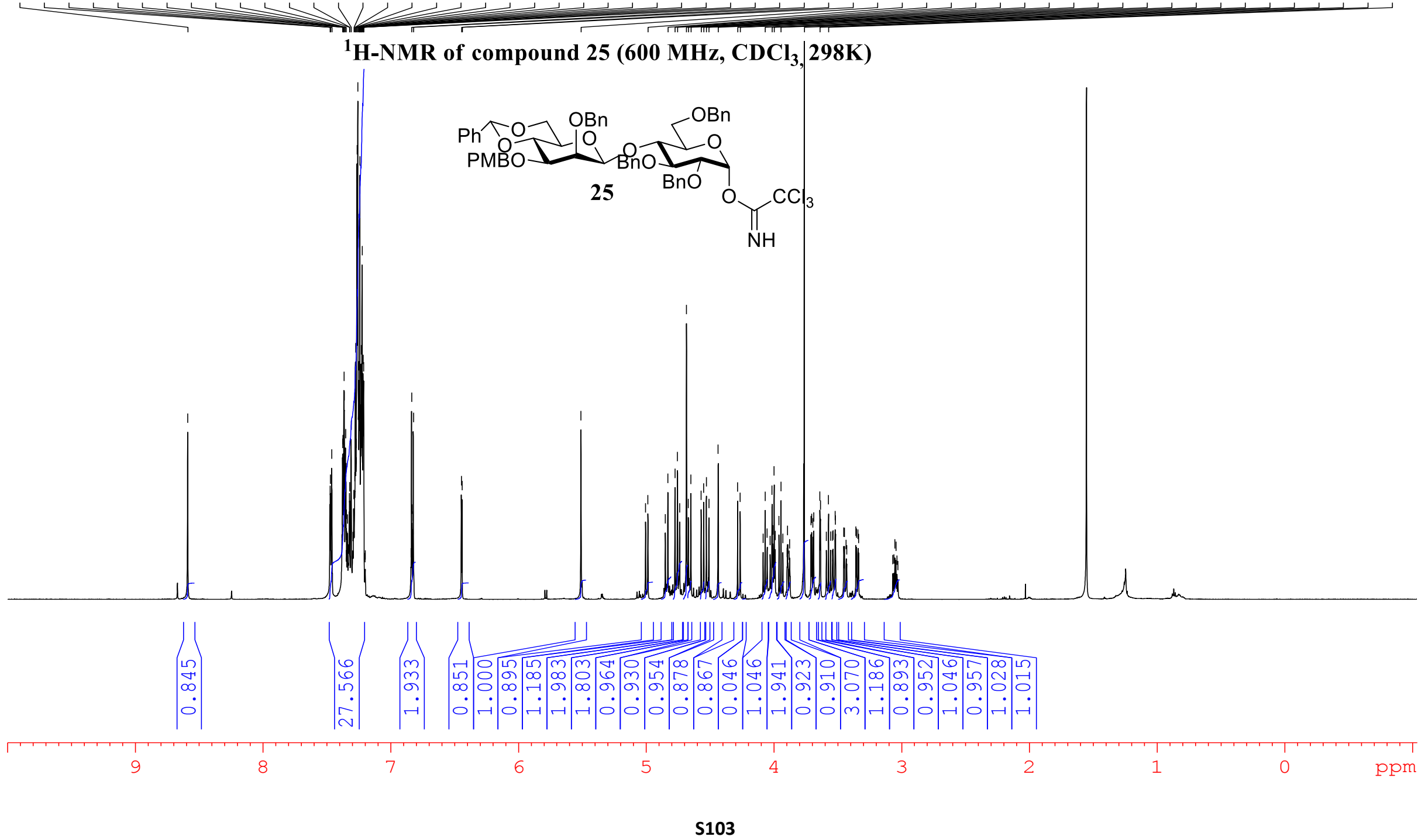




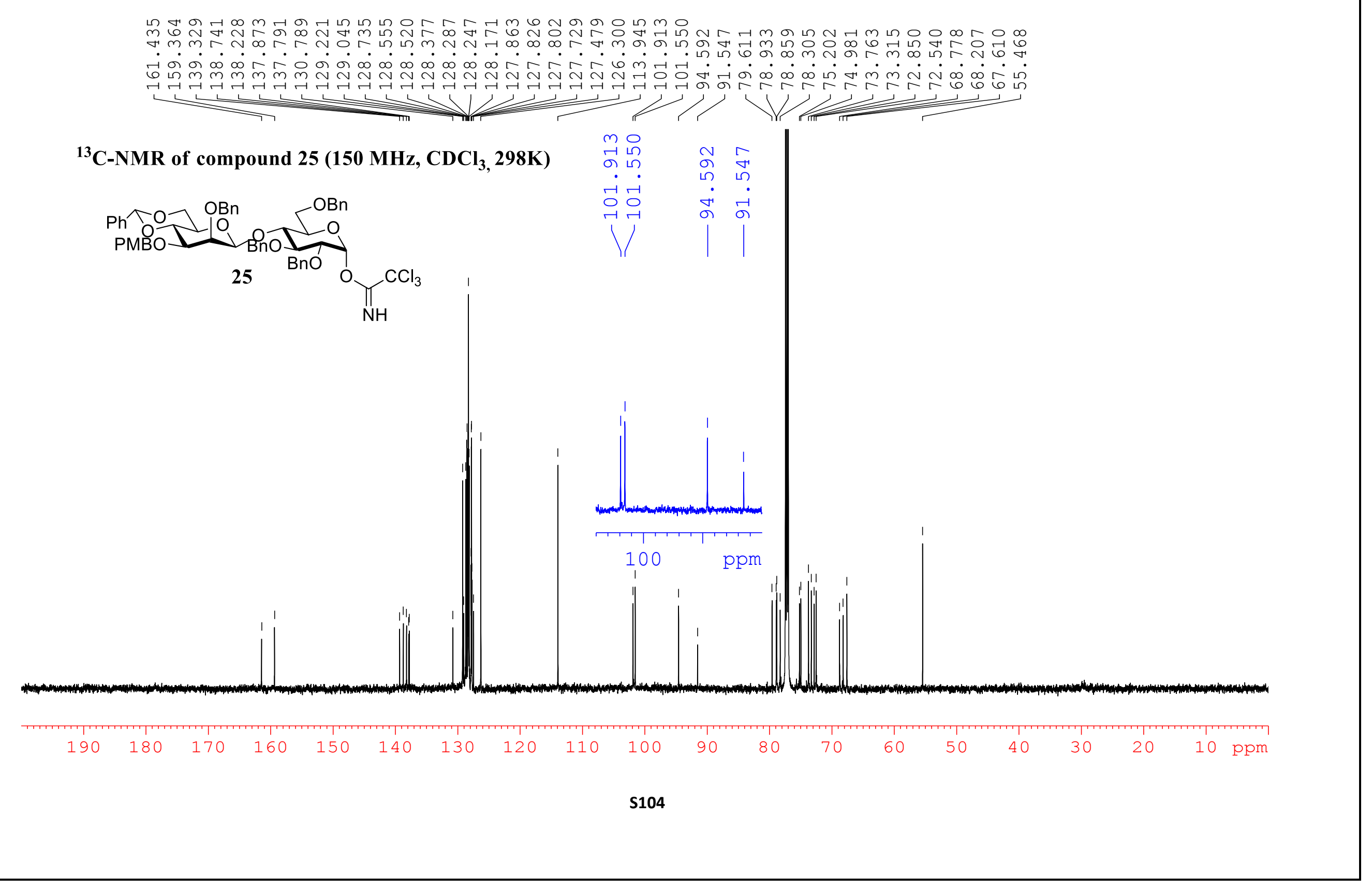




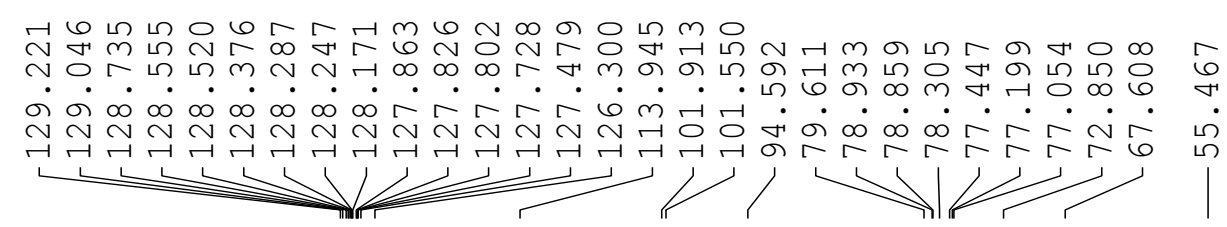

DEPT 135 NMR of compound 25 (150 $\left.\mathrm{MHz}, \mathrm{CDCl}_{3}, 298 \mathrm{~K}\right)$

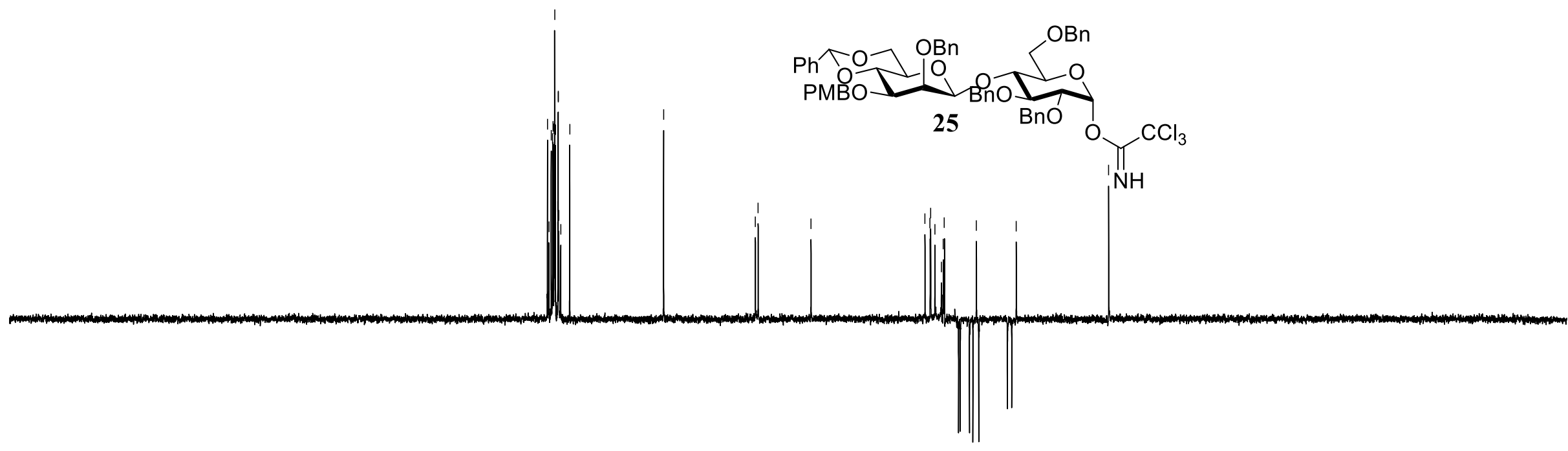

$\begin{array}{llllllllllllllllllllll}190 & 180 & 170 & 160 & 150 & 140 & 130 & 120 & 110 & 100 & 90 & 80 & 70 & 60 & 50 & 40 & 30 & 20 & 10 & \text { ppm }\end{array}$




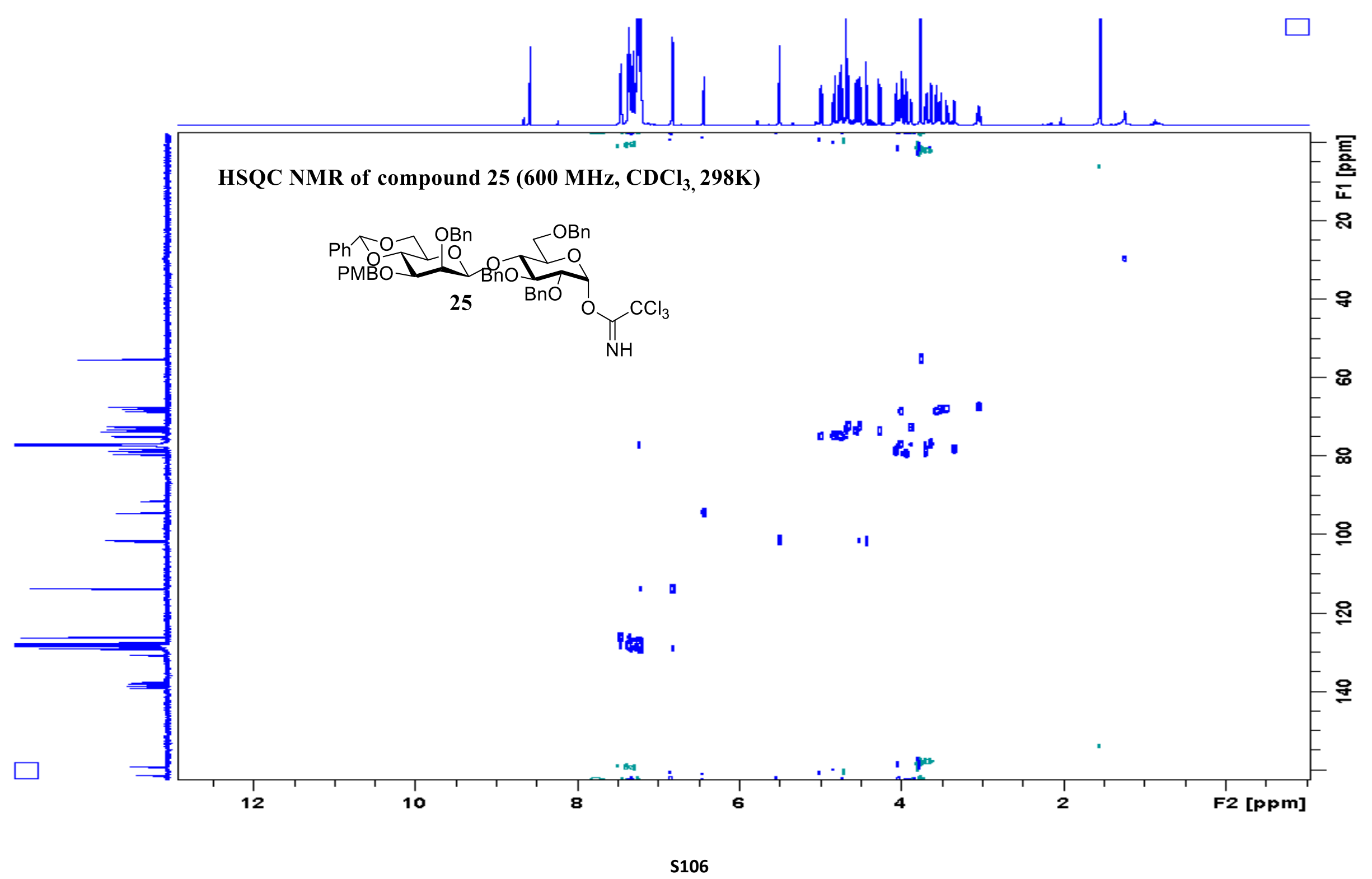




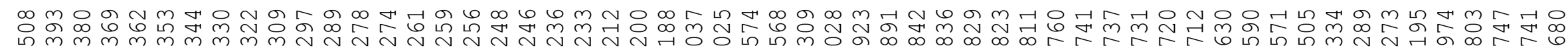

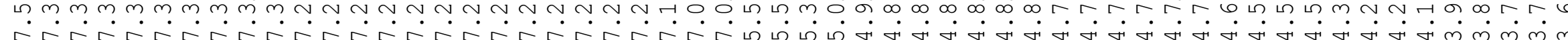

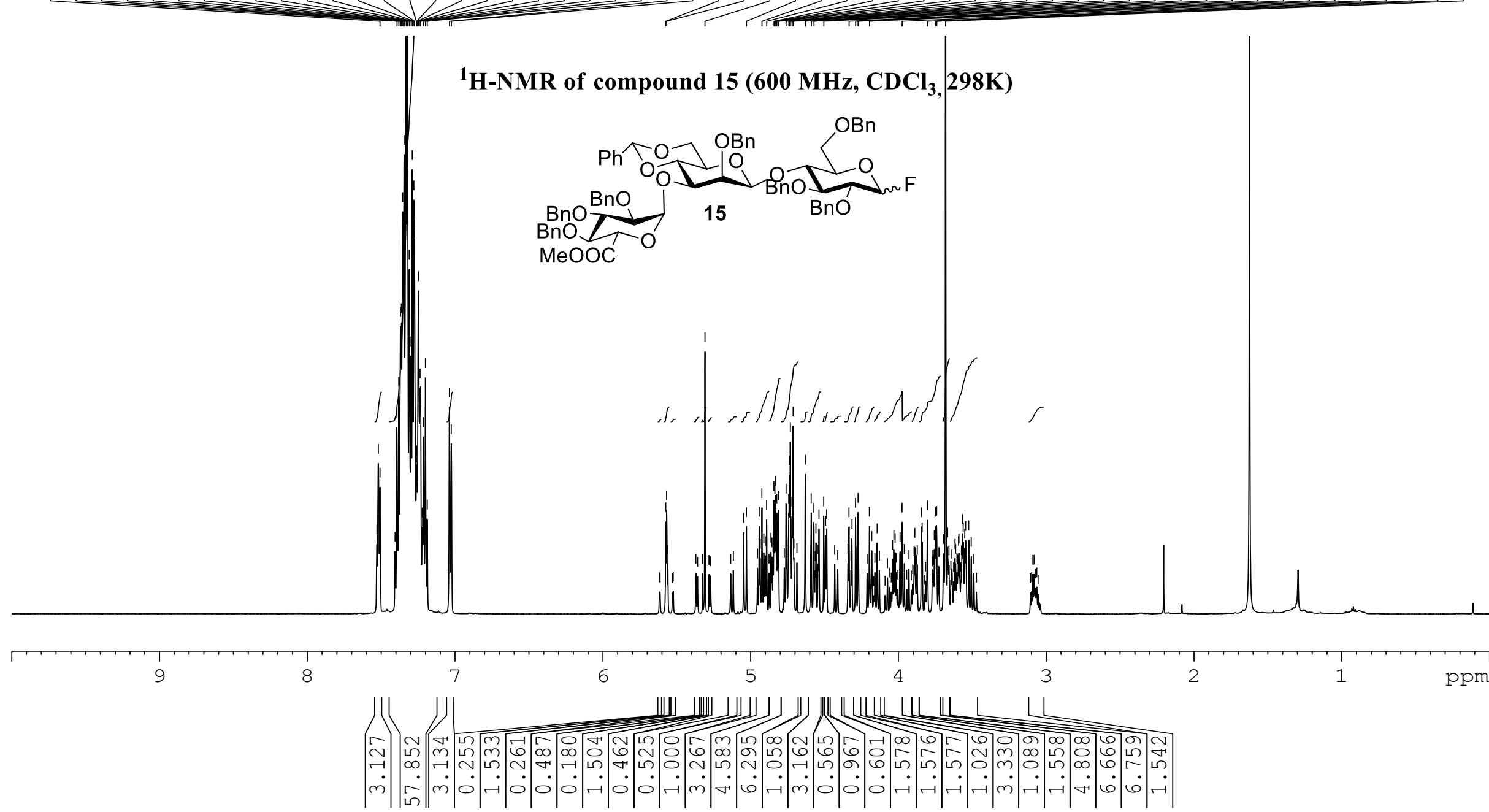

S107 


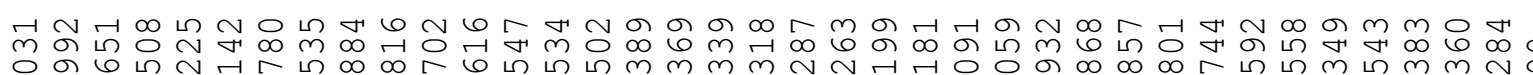

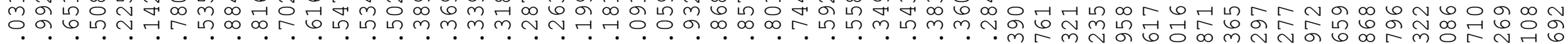

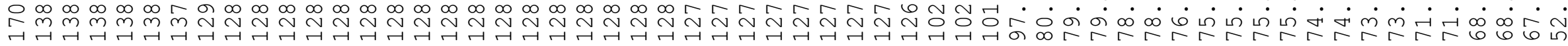

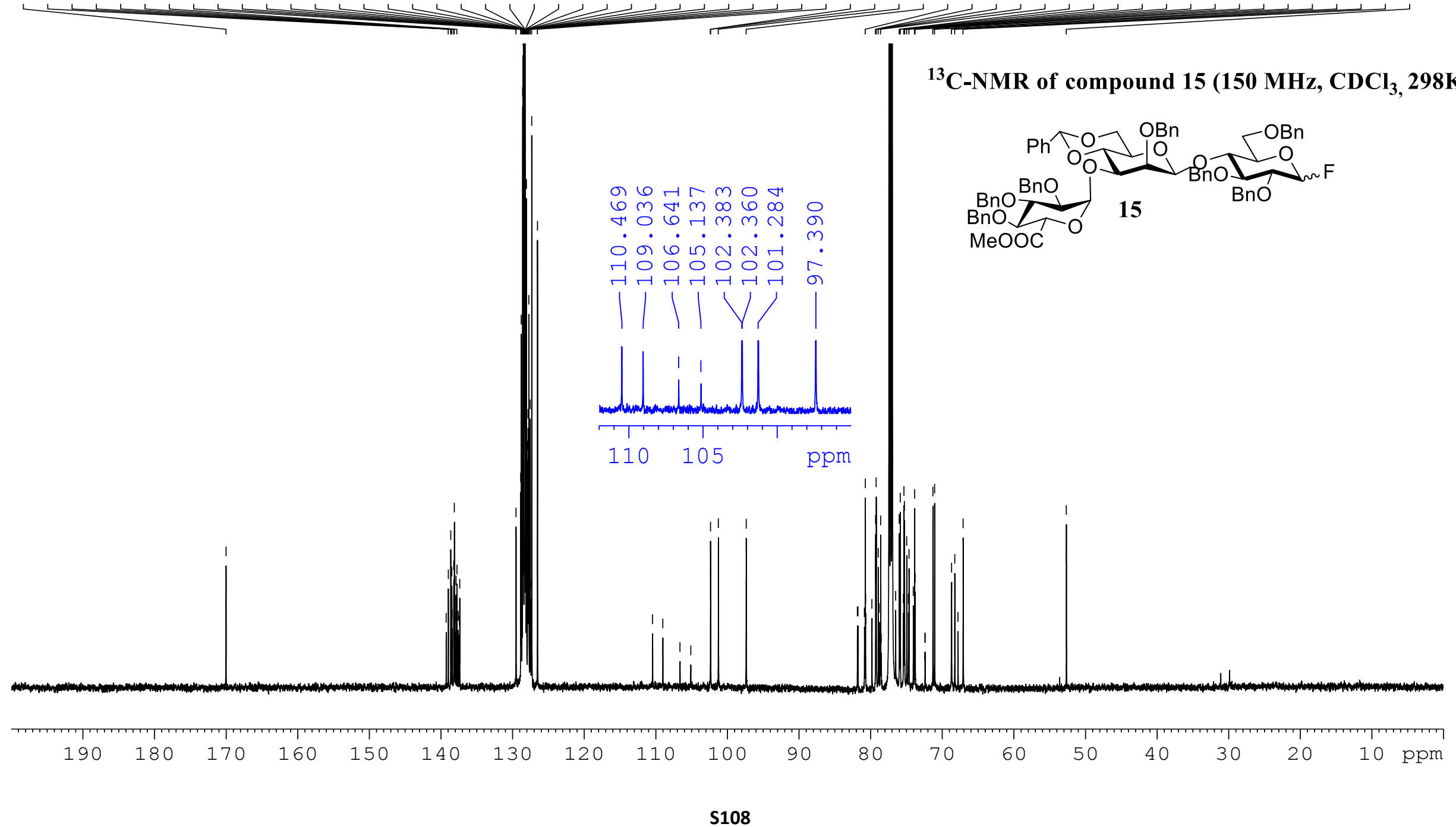




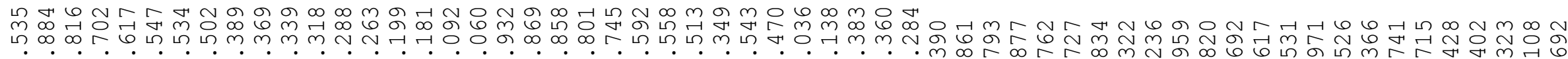
बi

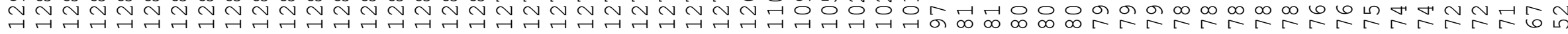

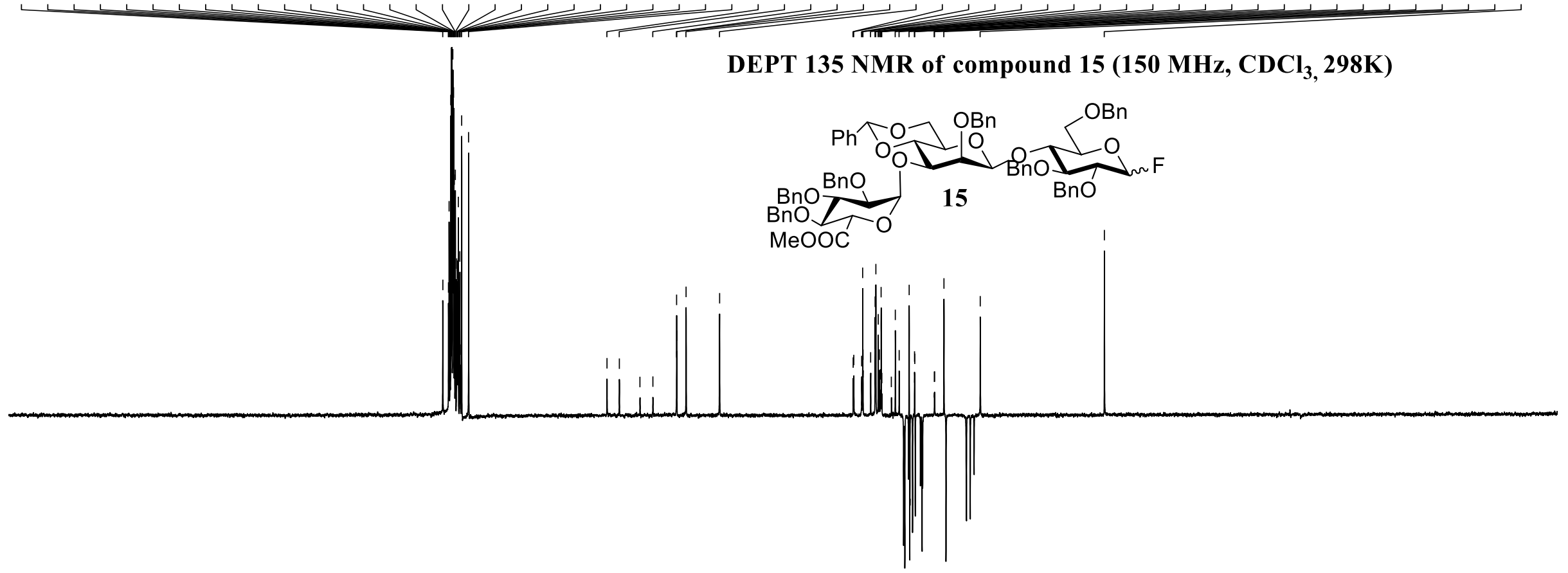

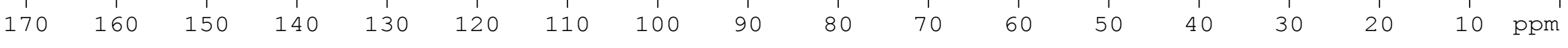




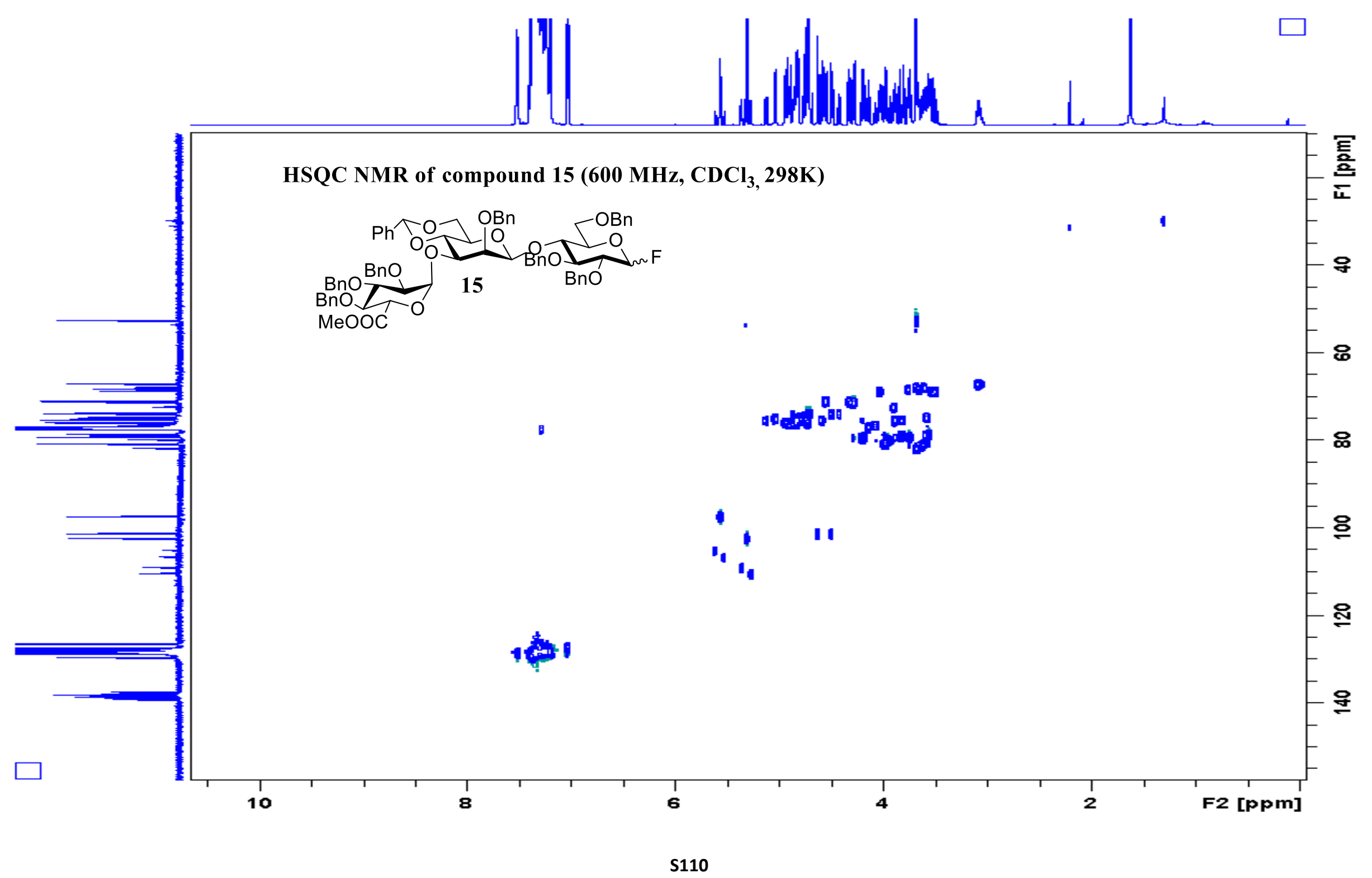




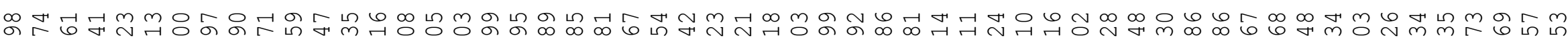

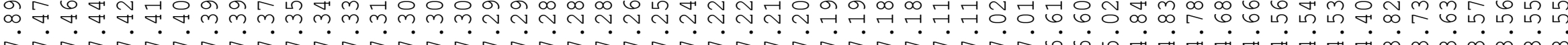

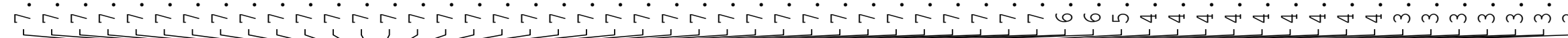

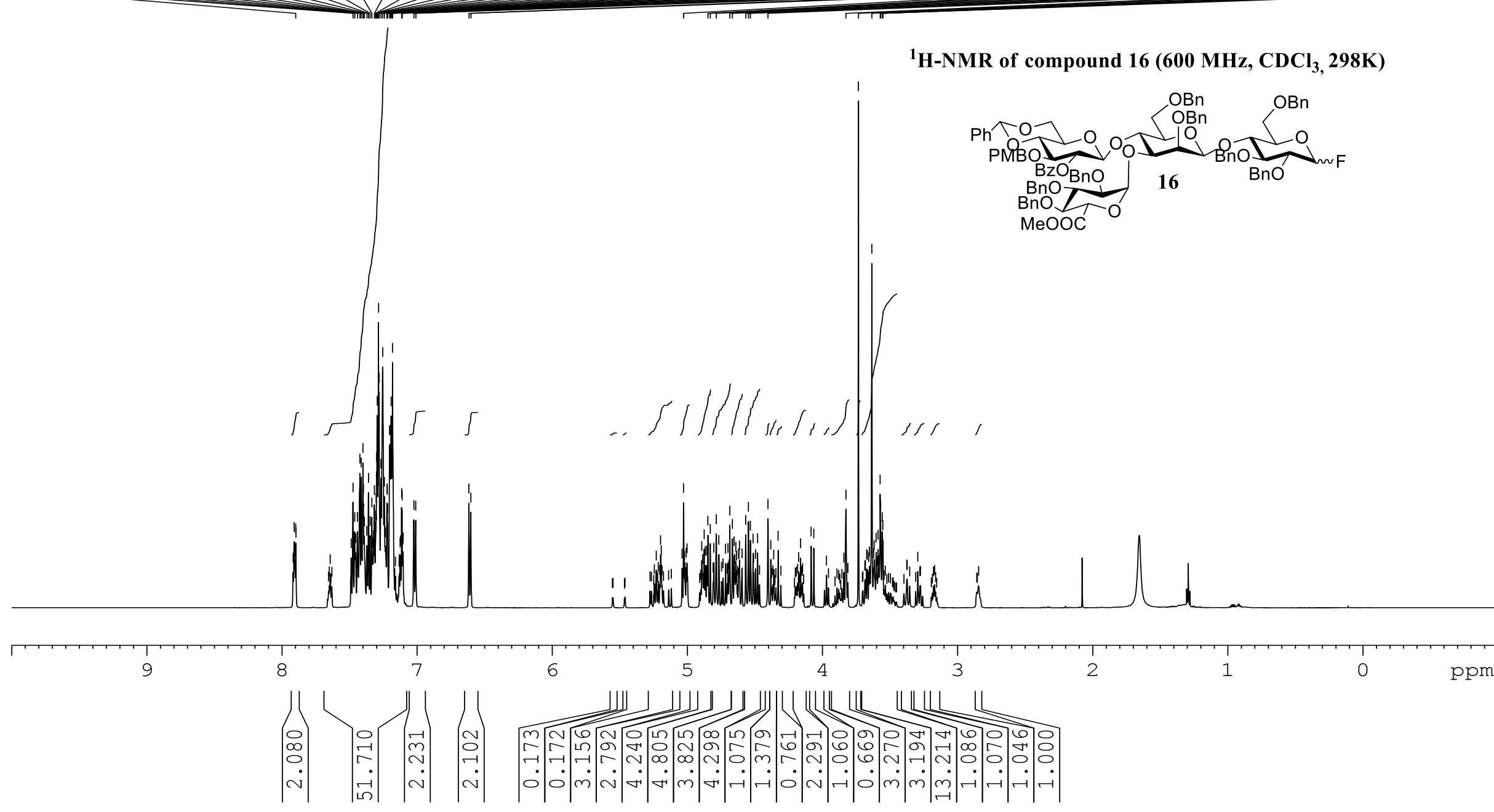




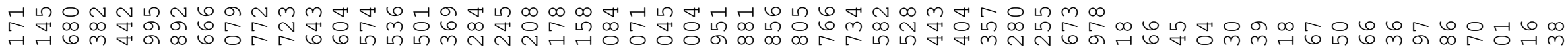

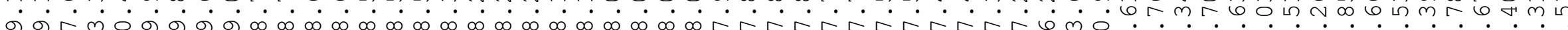

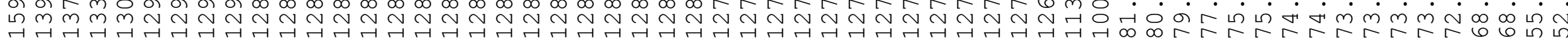

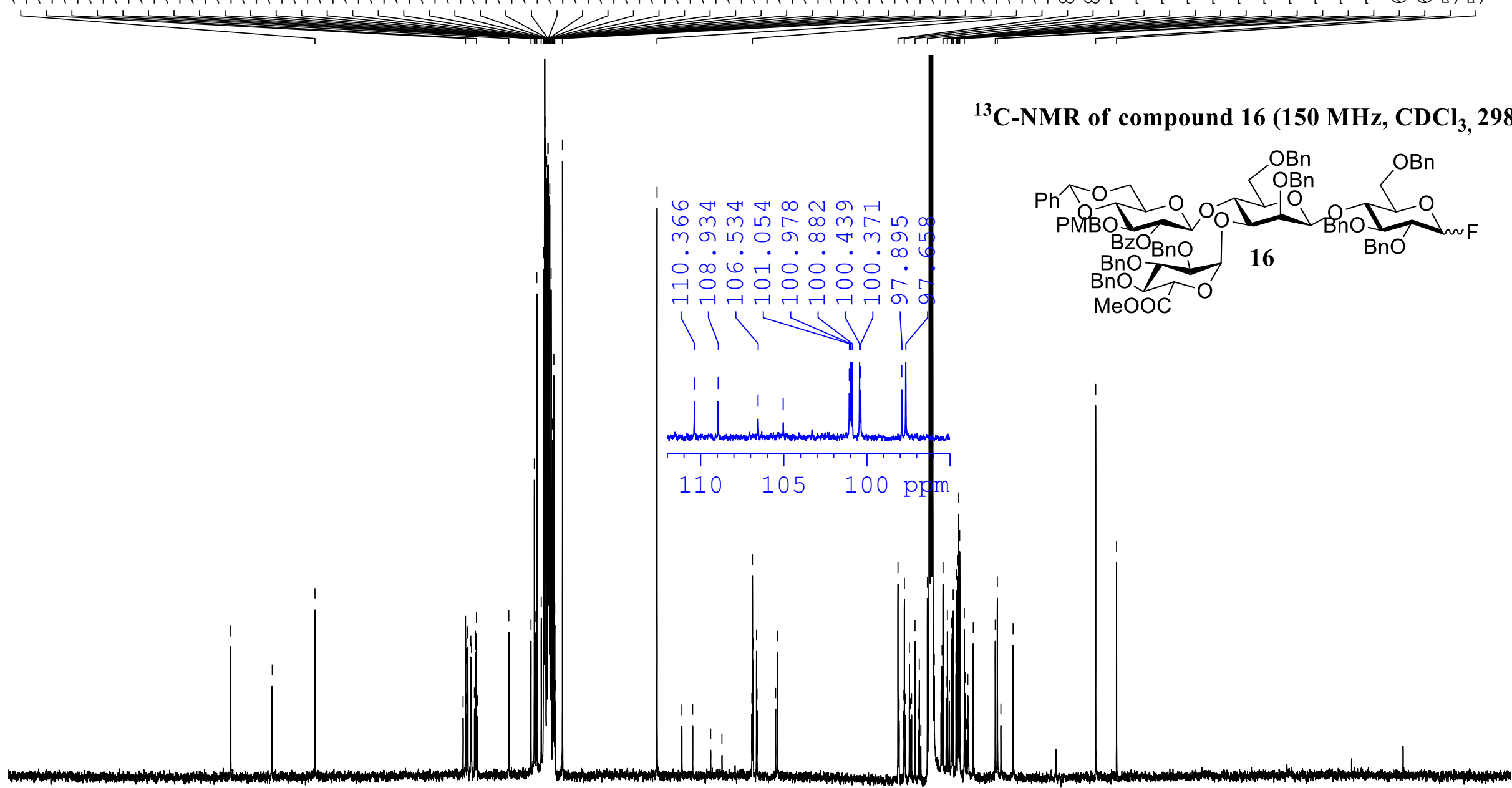




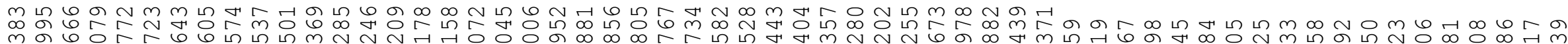
সंबंன்

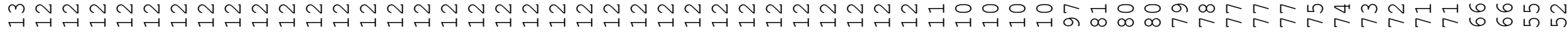

\section{DEPT 135 NMR of compound $16\left(150 \mathrm{MHz}, \mathrm{CDCl}_{3}, 298 \mathrm{~K}\right)$}

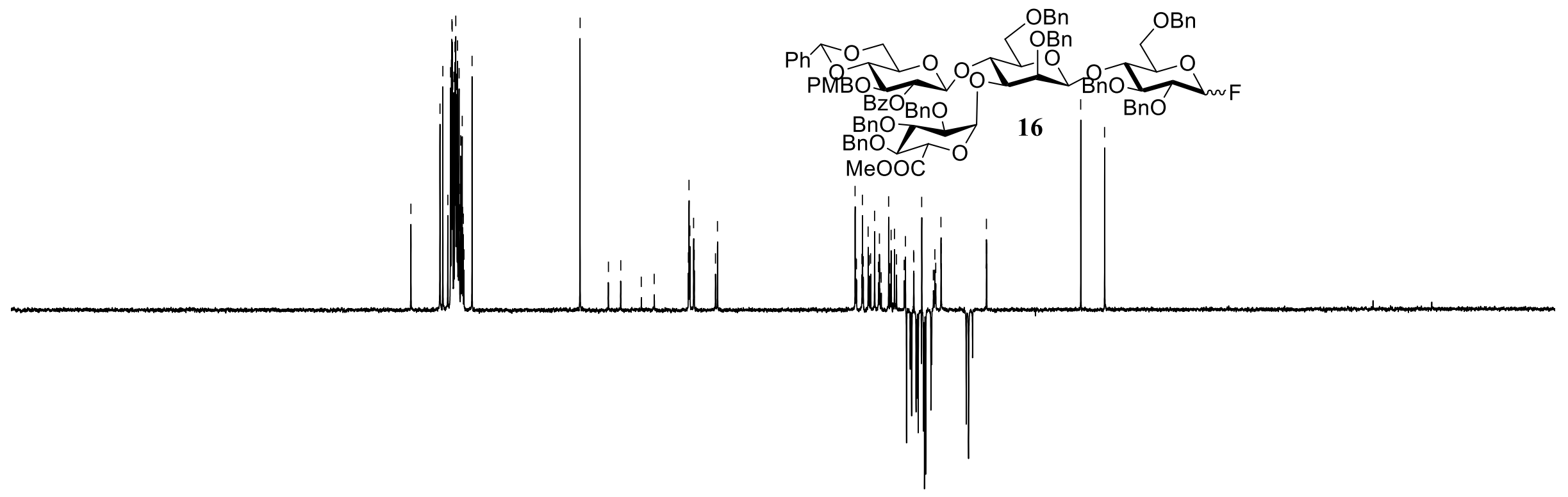

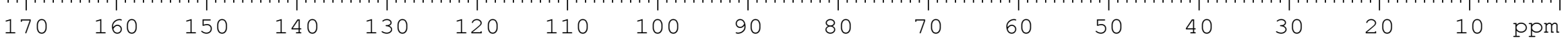




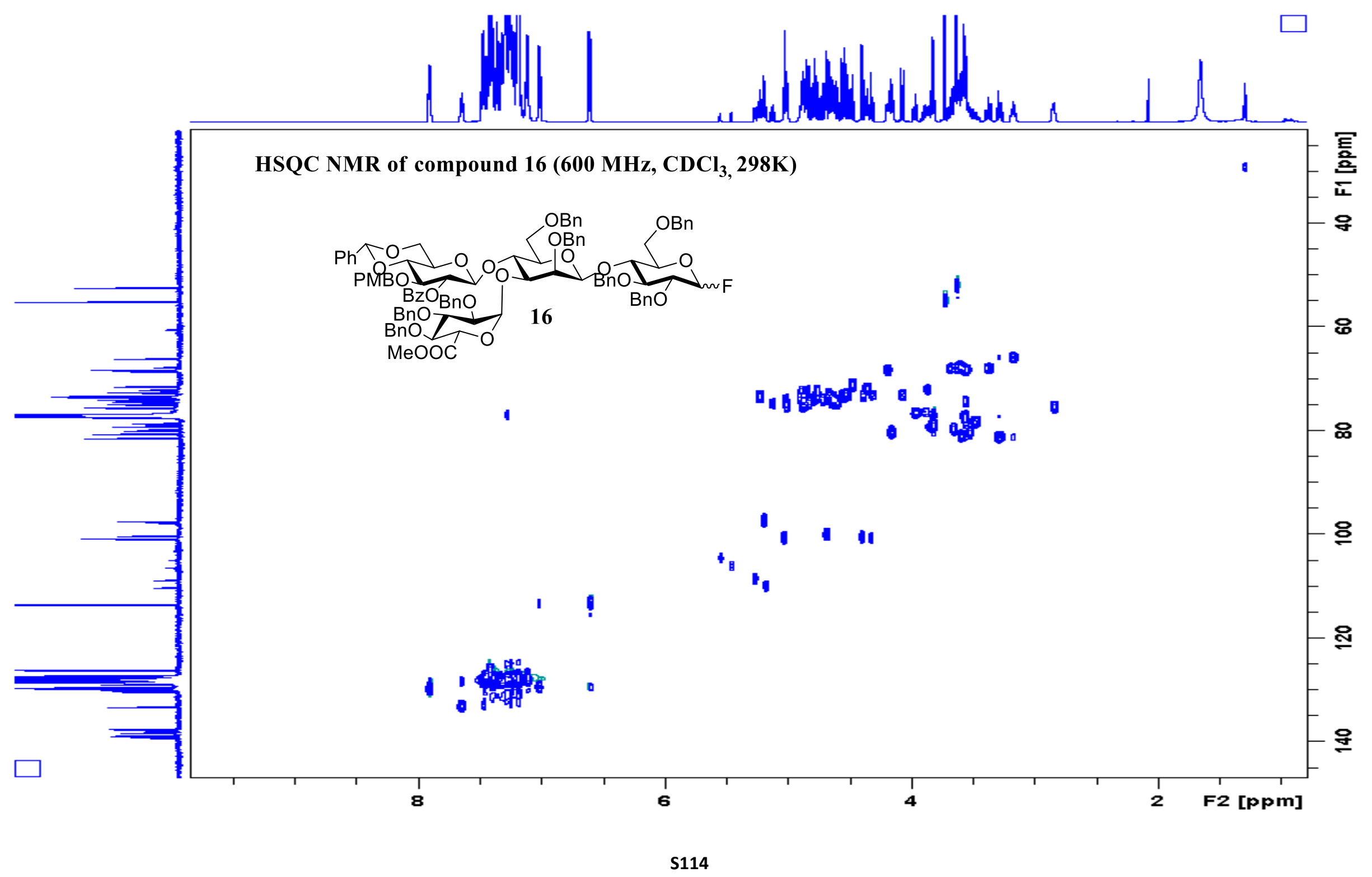




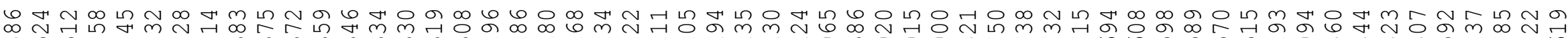

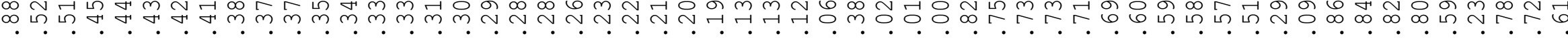

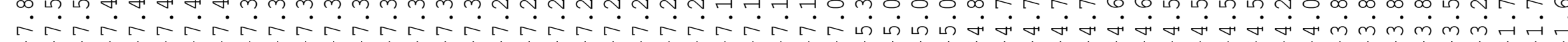

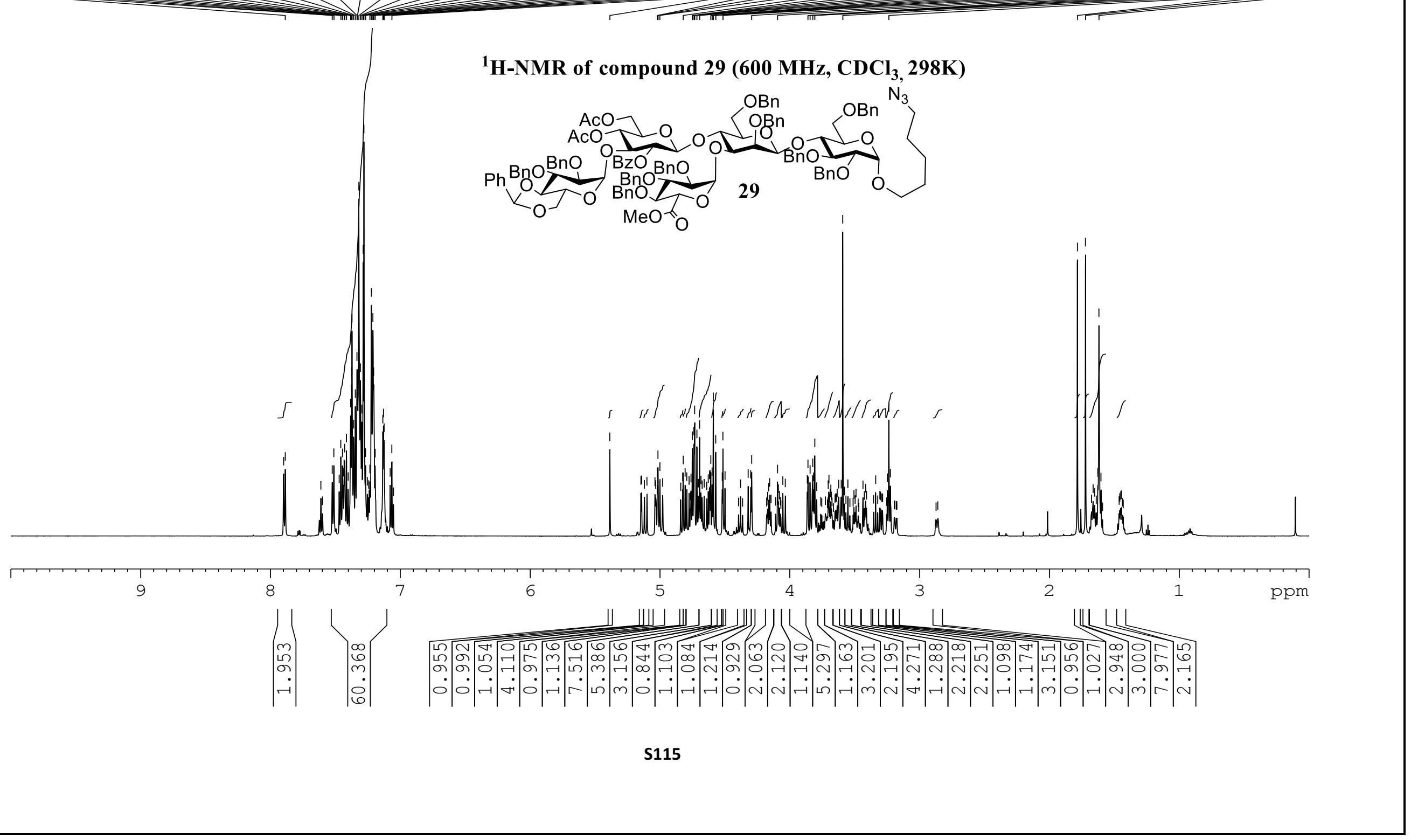




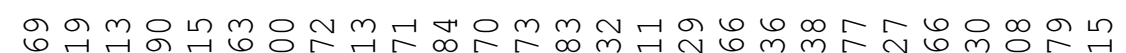
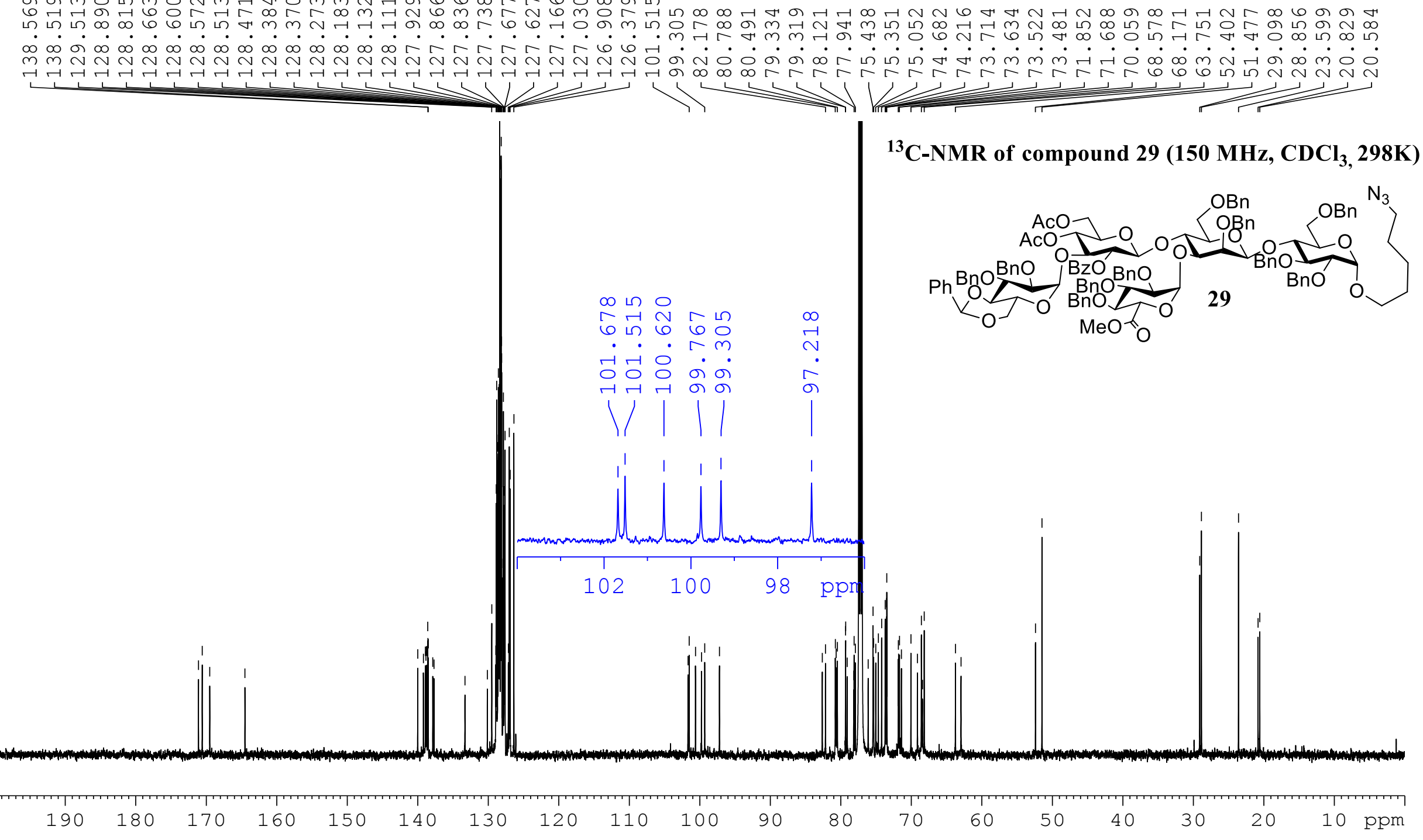

S116 


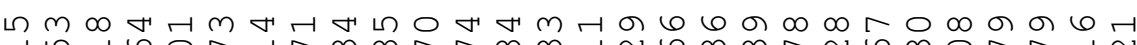

H .

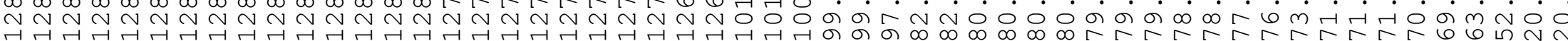

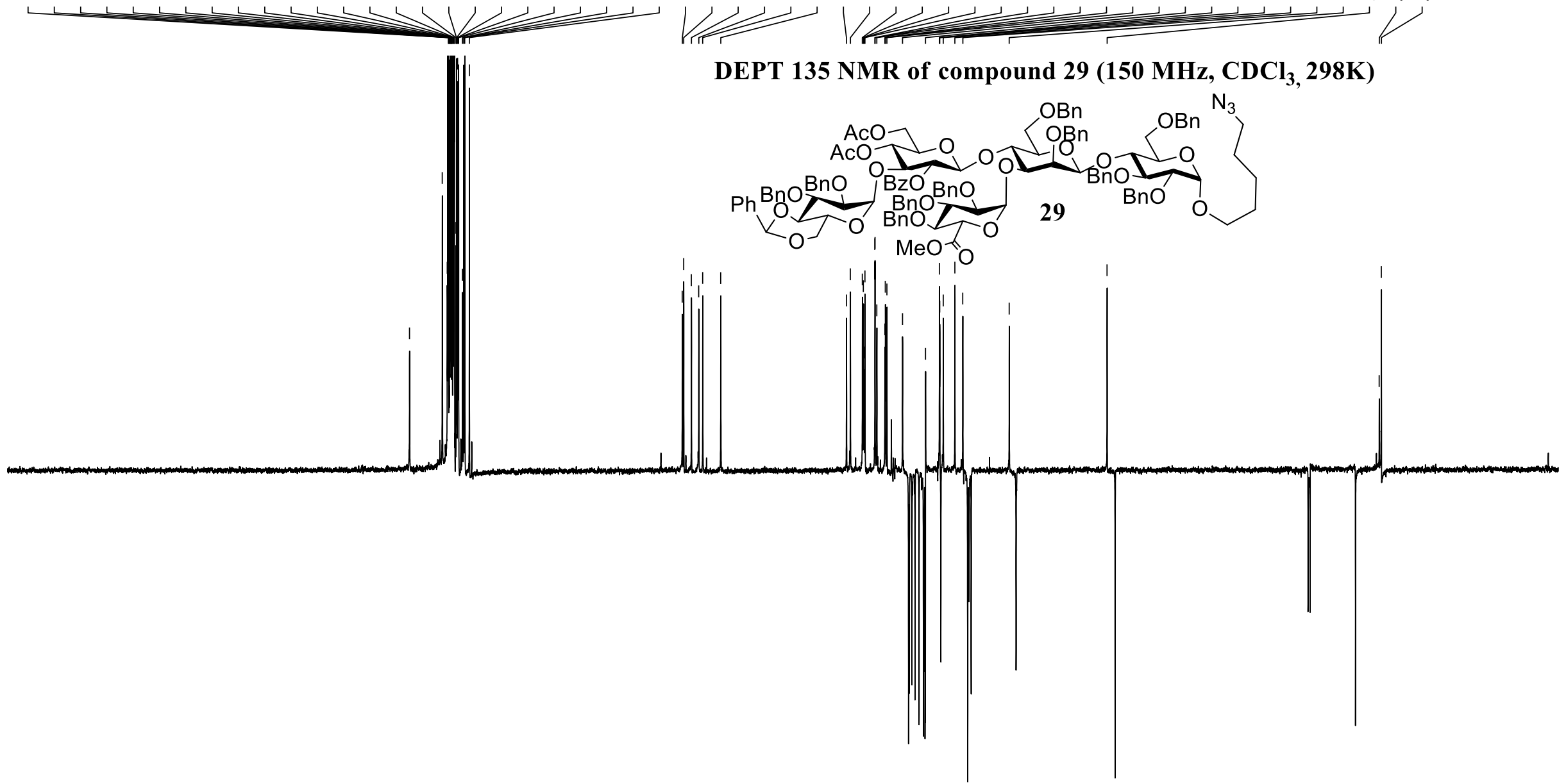

S117 


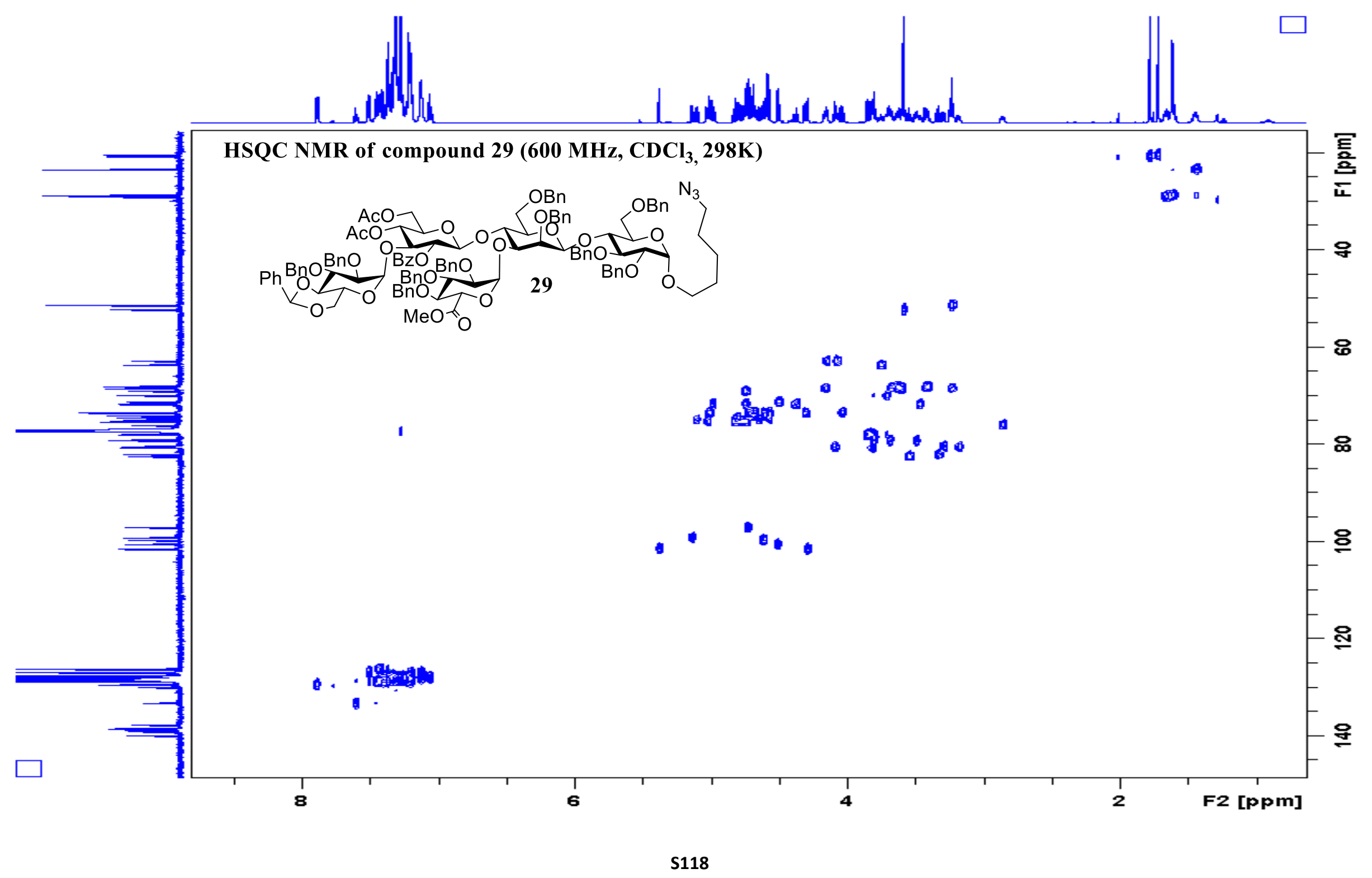




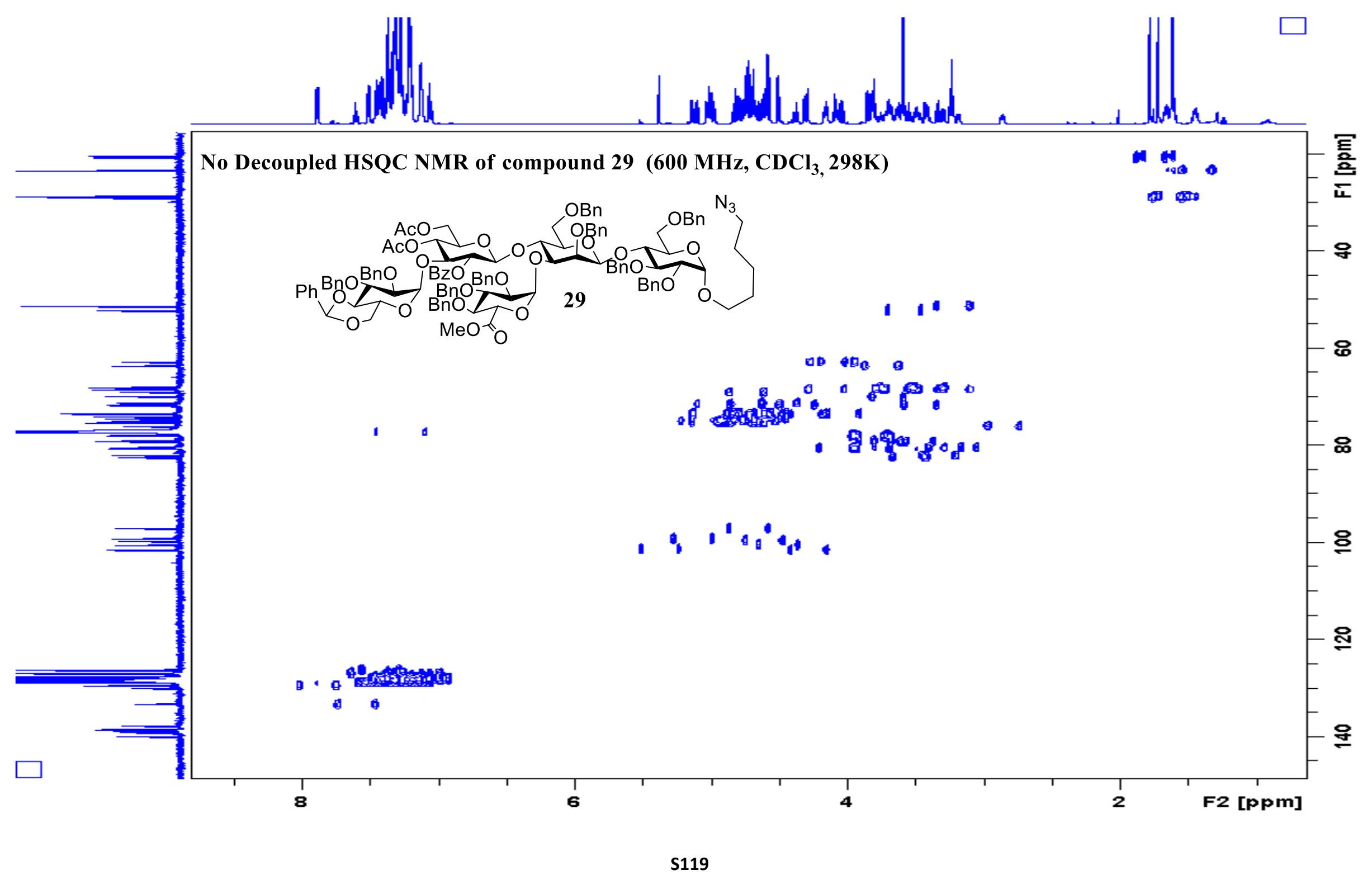




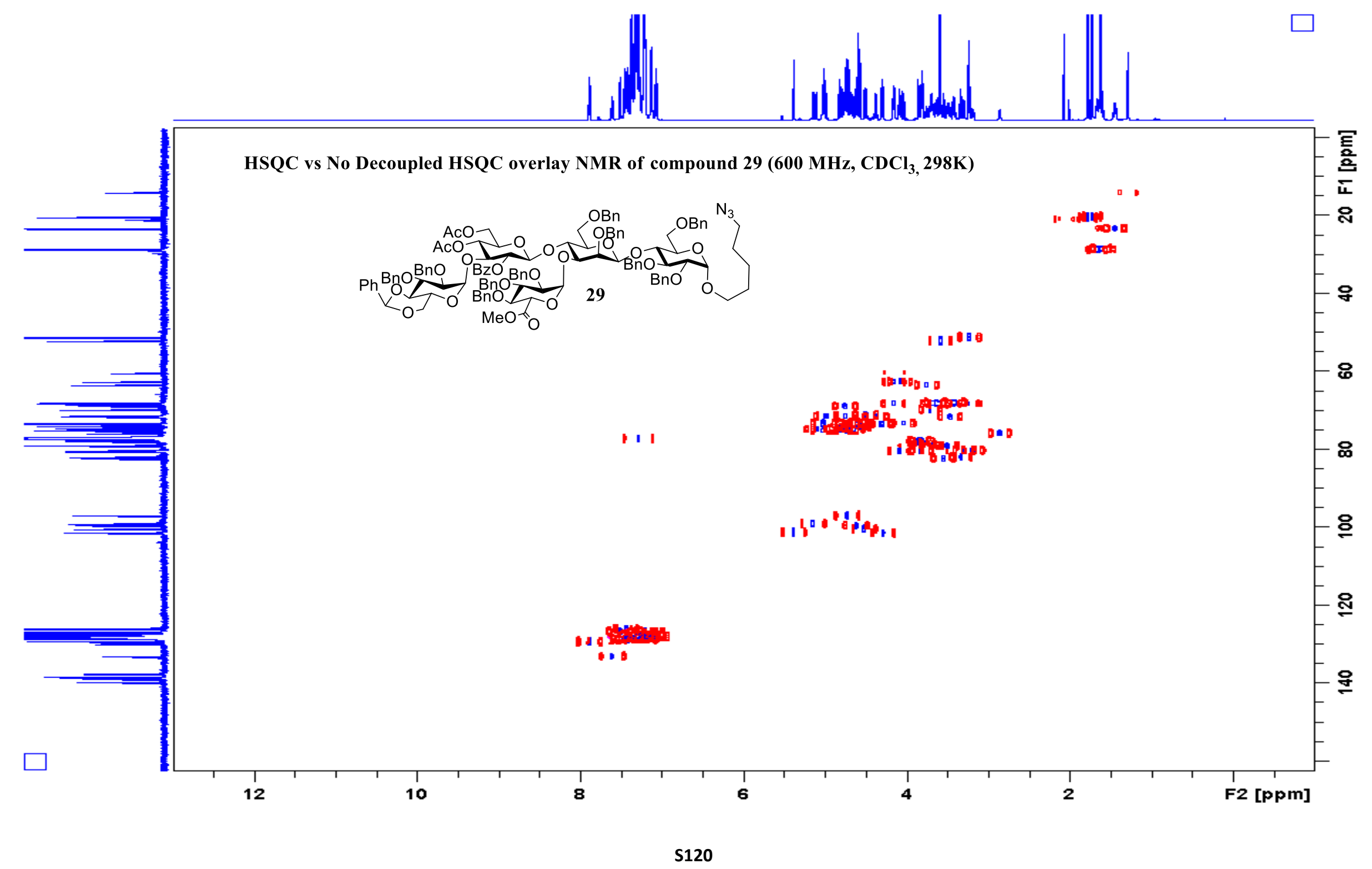




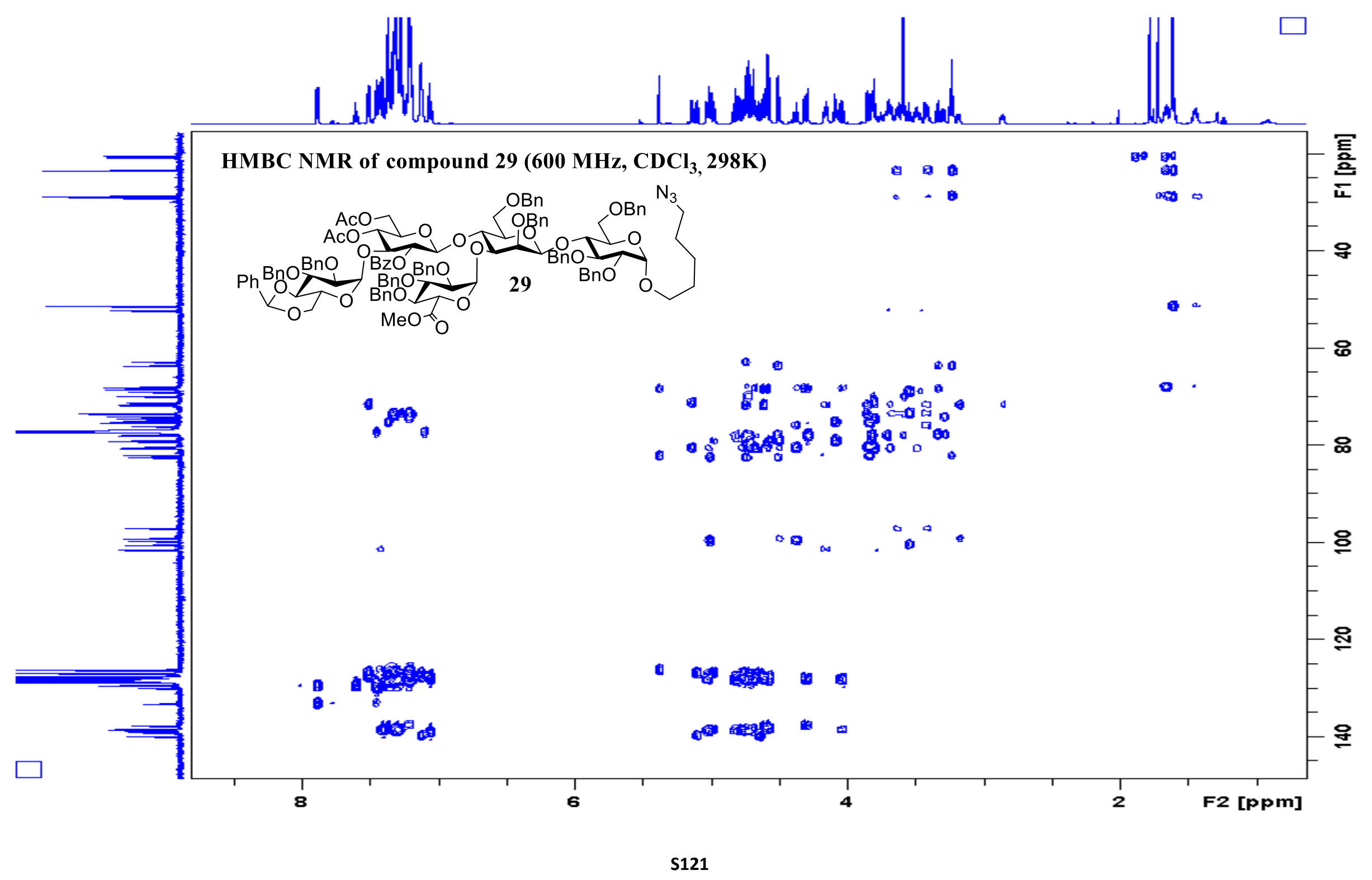




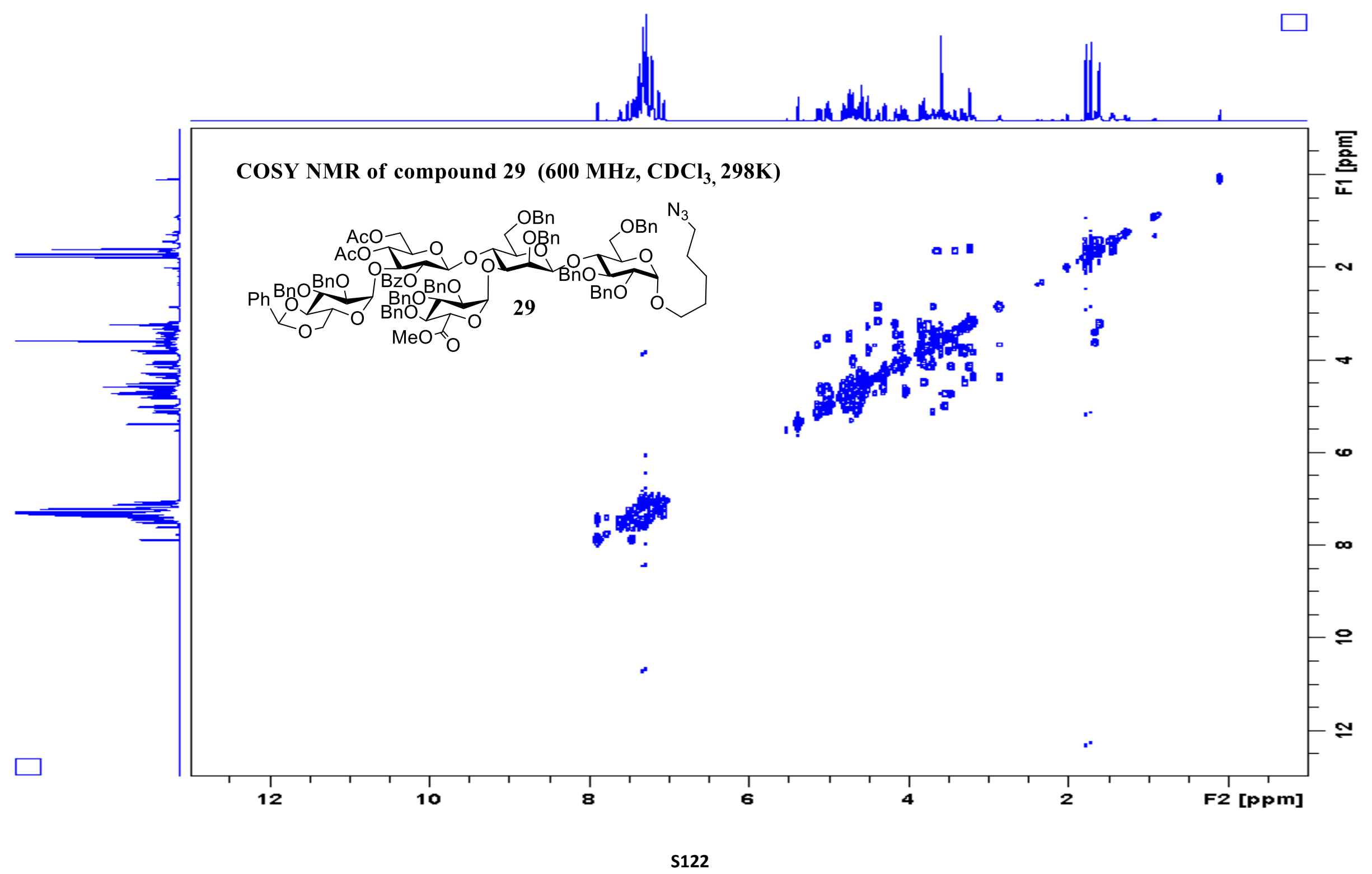




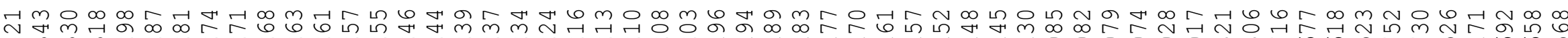

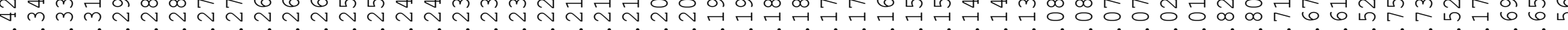

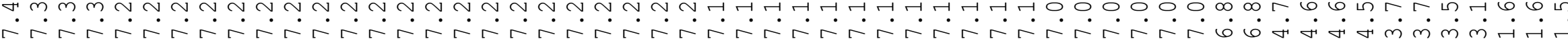

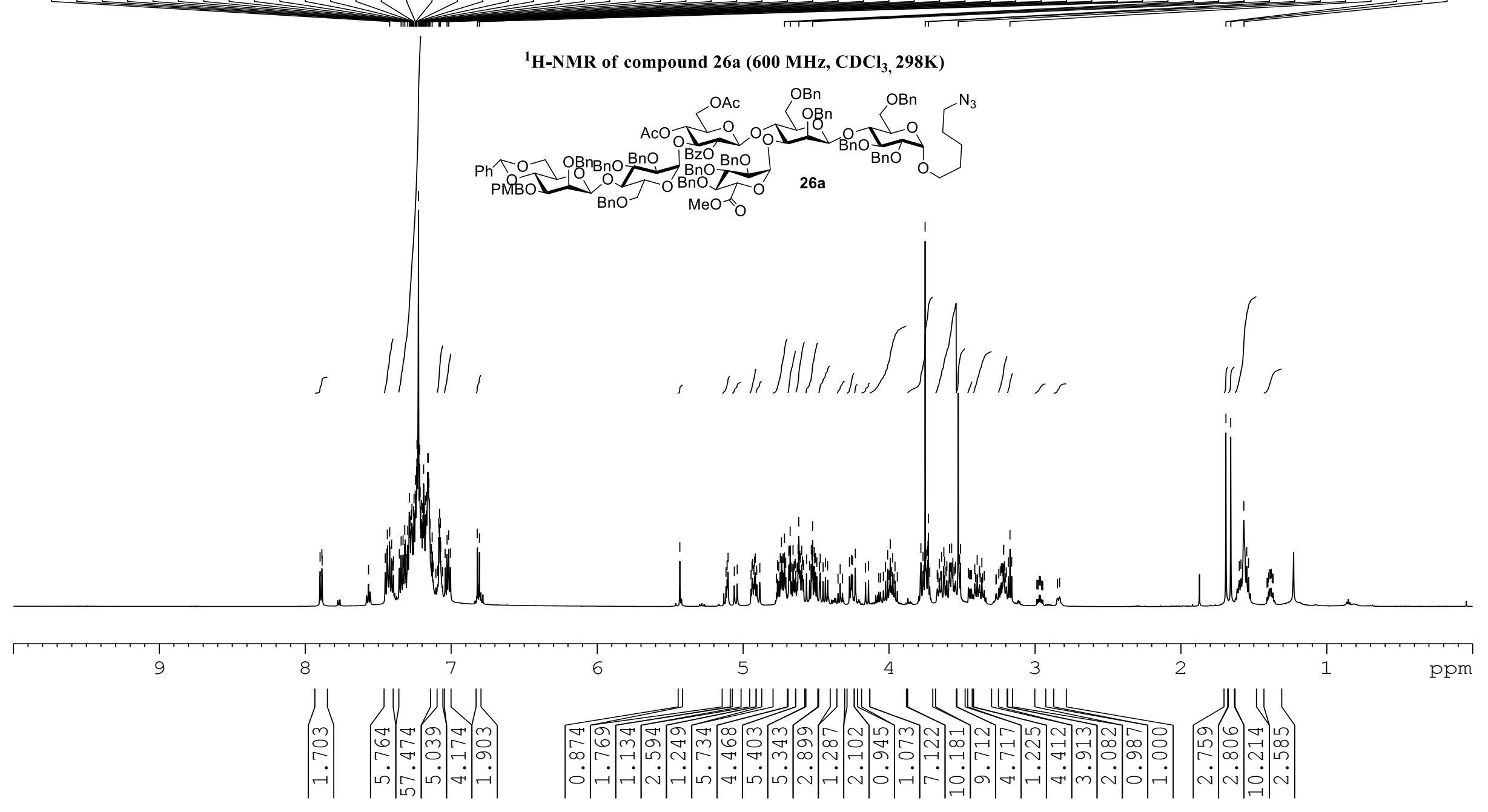




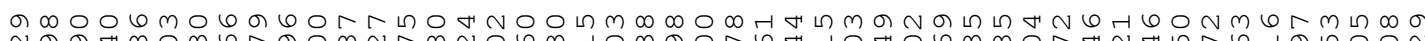

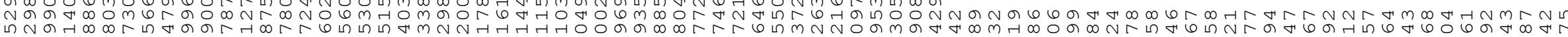

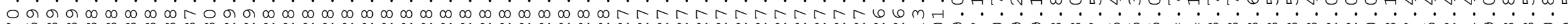

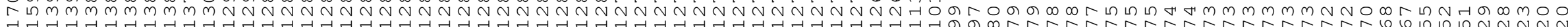

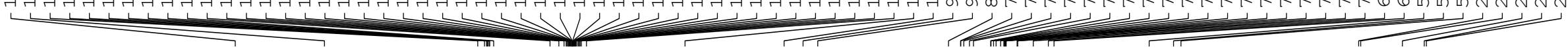

${ }^{13} \mathrm{C}-\mathrm{NMR}$ of compound 26a (150 MHz, $\left.\mathrm{CDCl}_{3}, 298 \mathrm{~K}\right)$

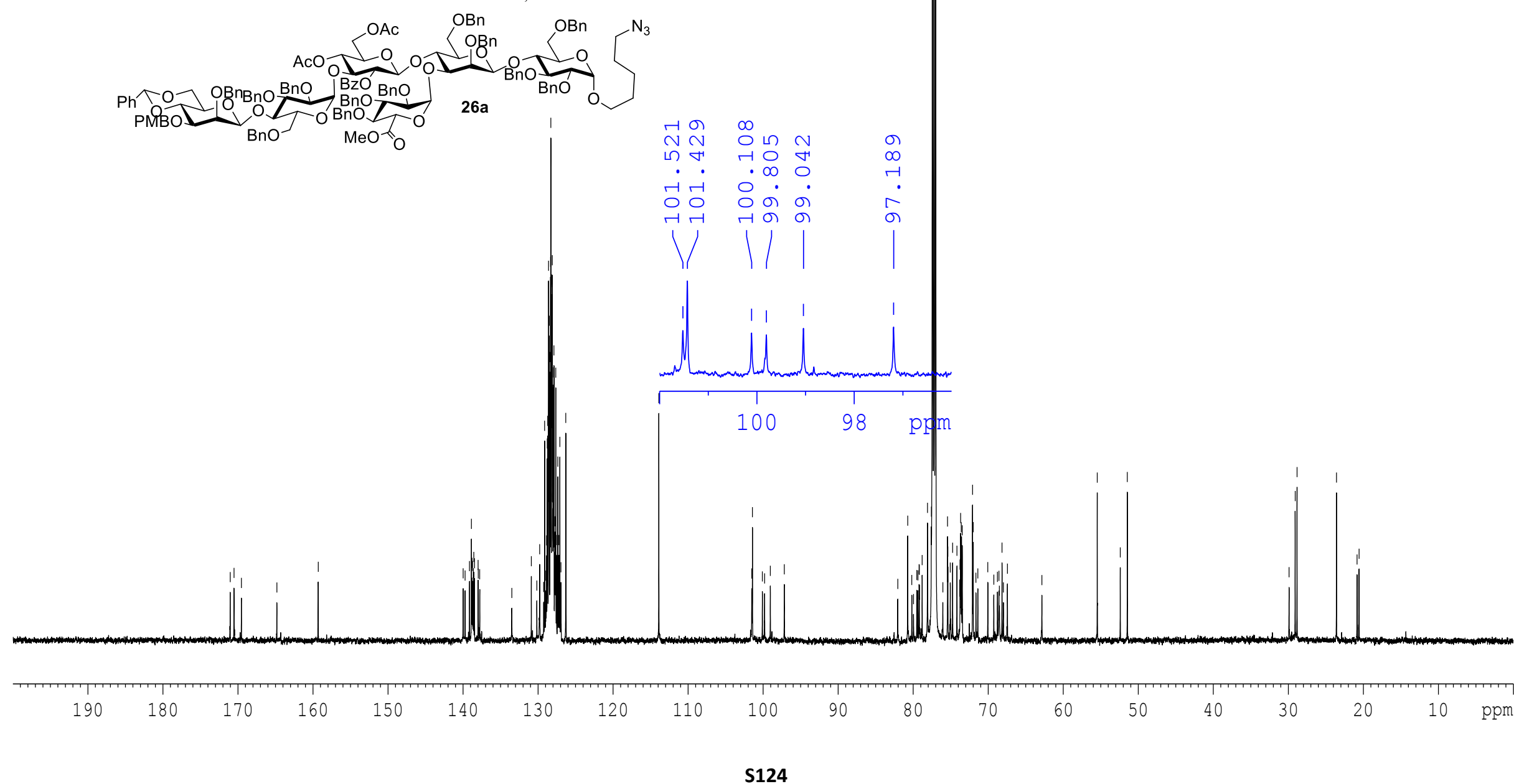


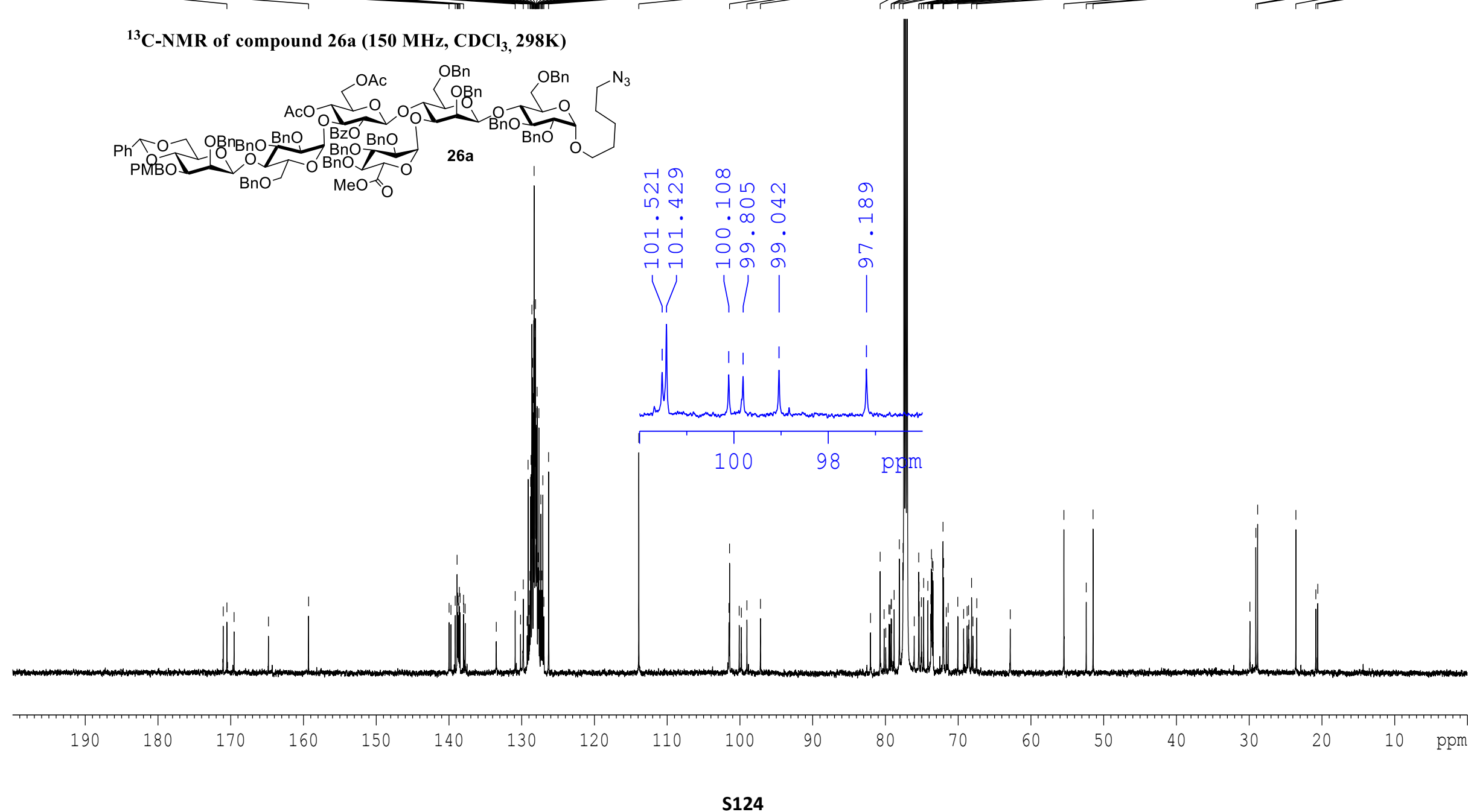
( 


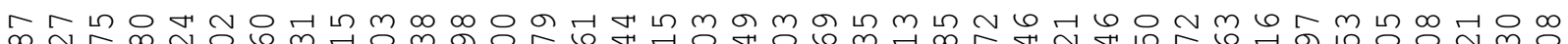

क 군

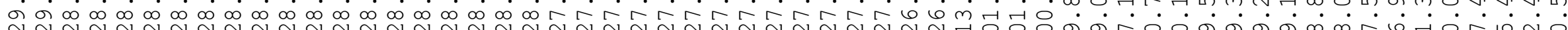

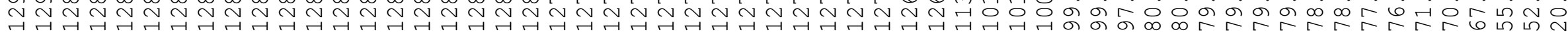

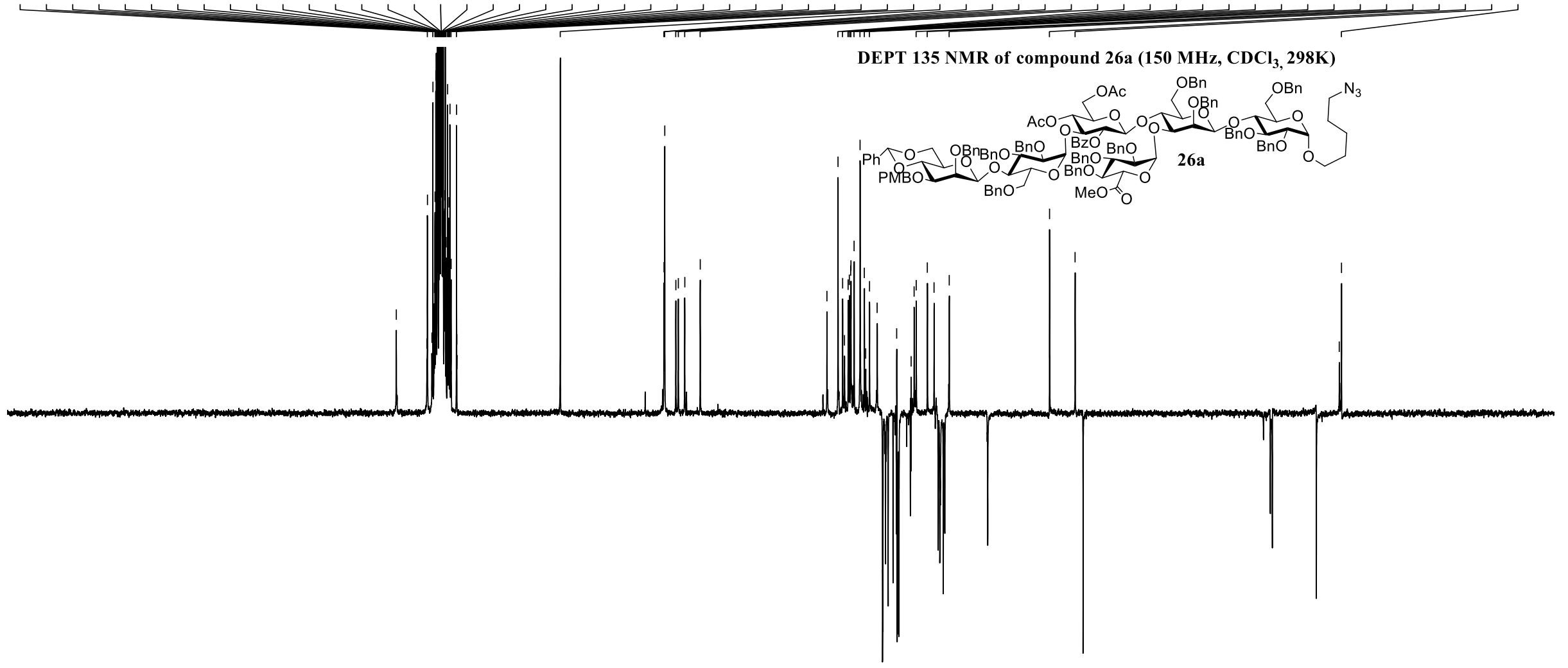

$\begin{array}{llllllllllllllllll}170 & 160 & 150 & 140 & 130 & 120 & 110 & 100 & 90 & 80 & 70 & 60 & 50 & 40 & 30 & 20 & 10 & \text { ppm }\end{array}$




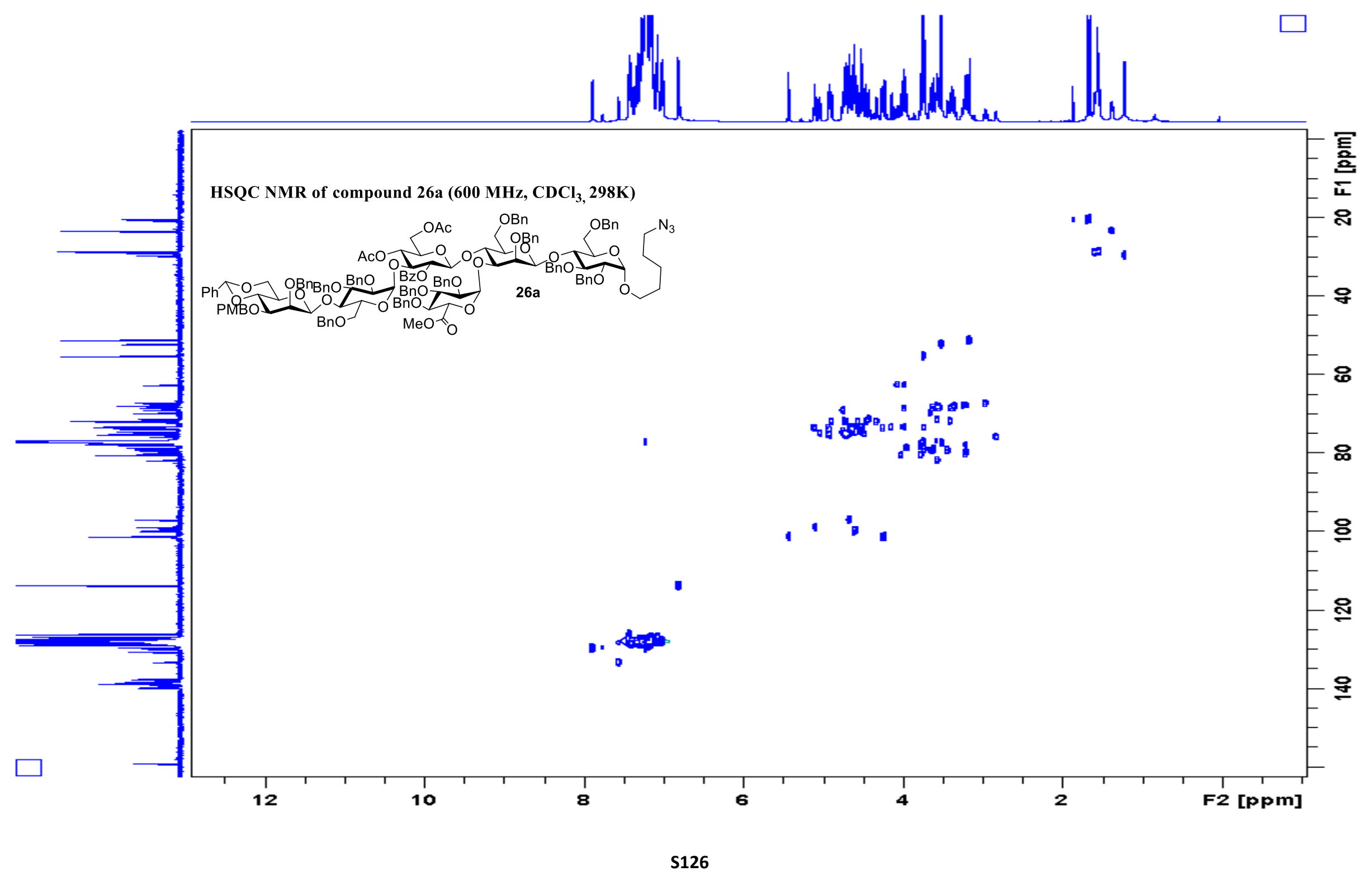




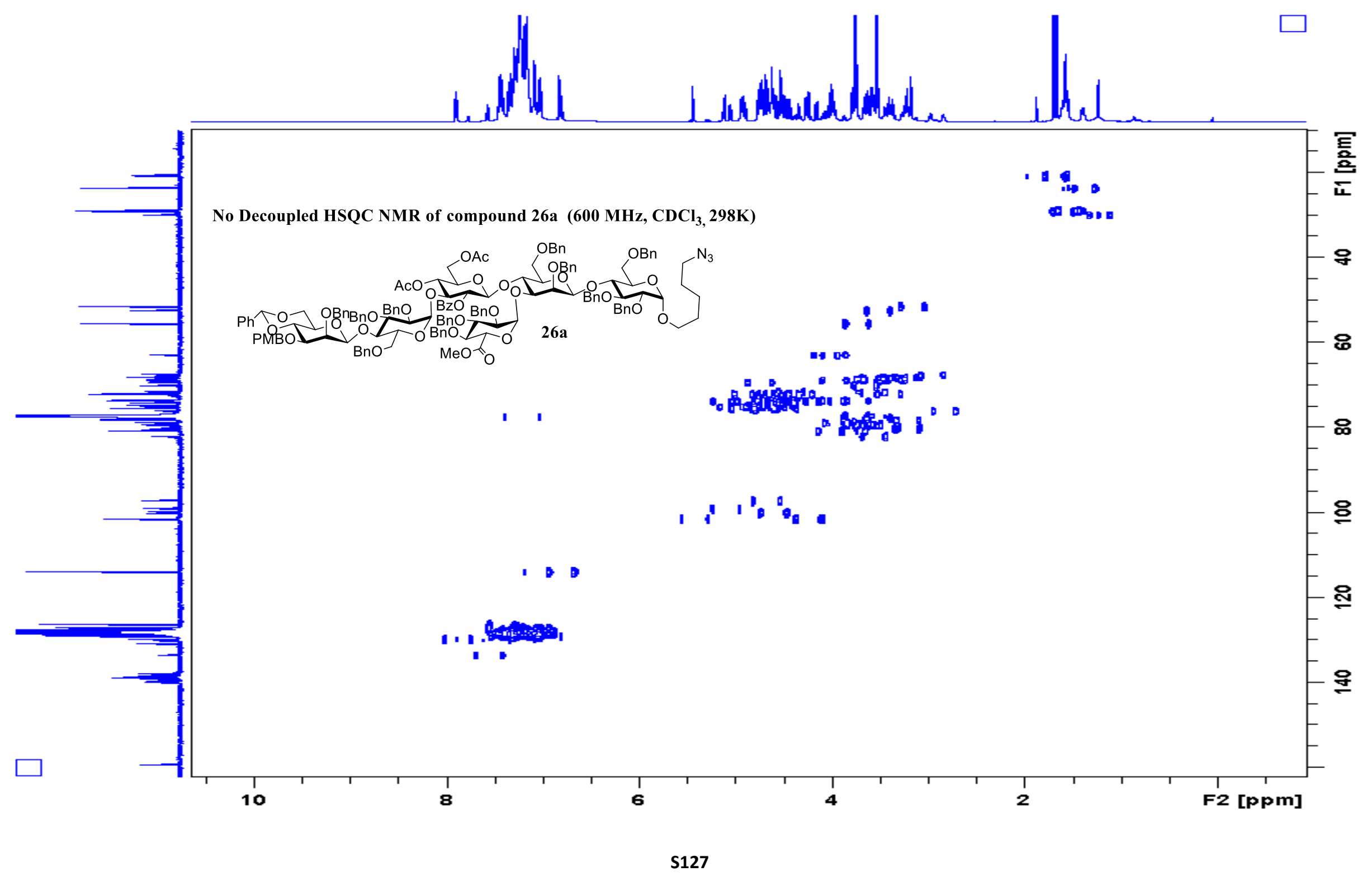




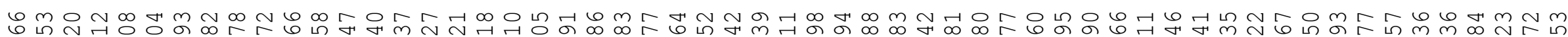

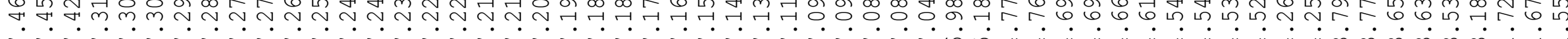

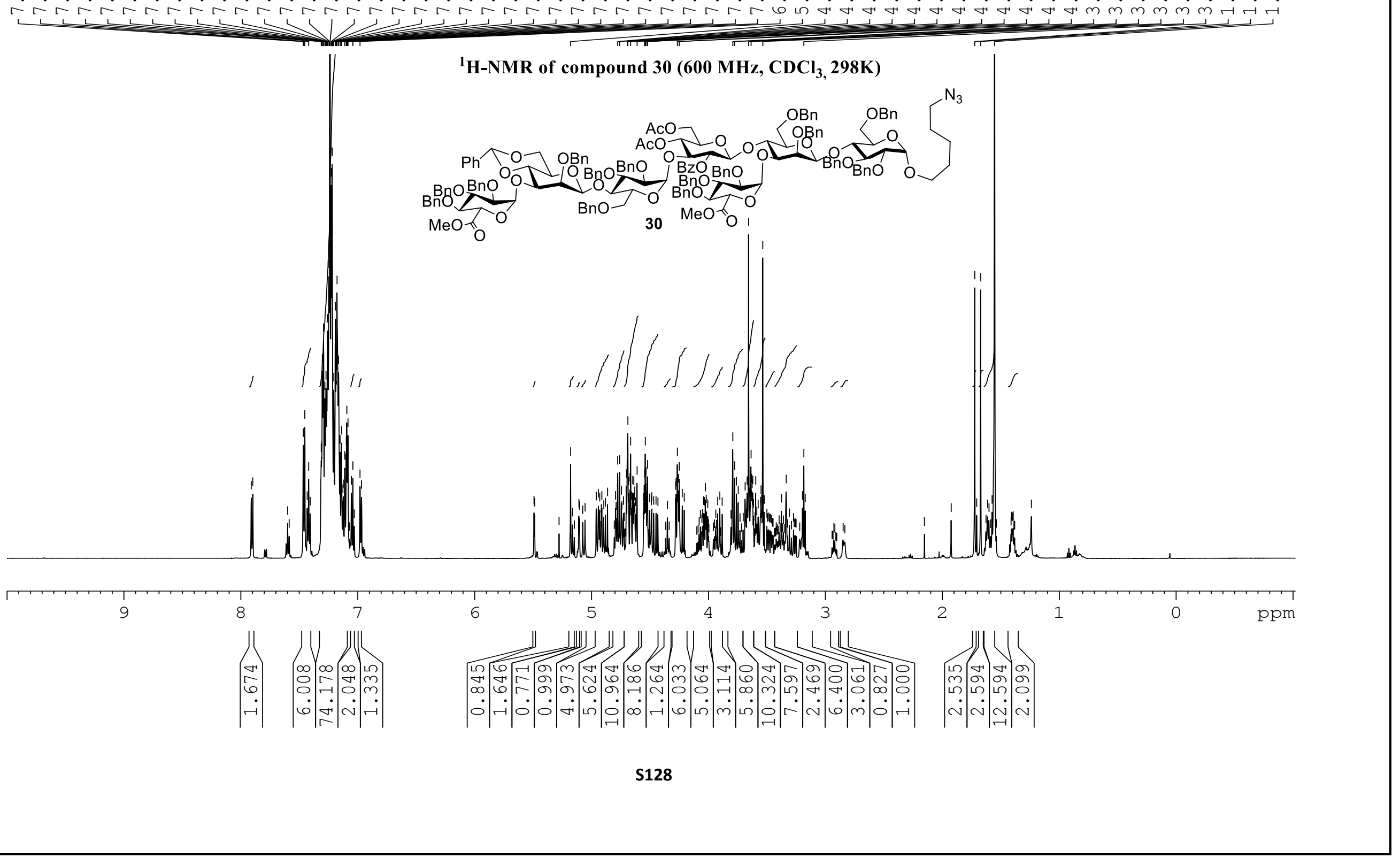




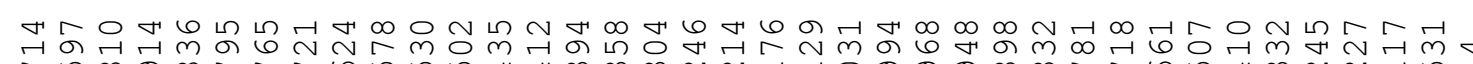

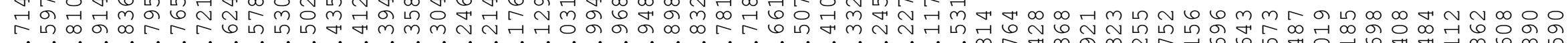

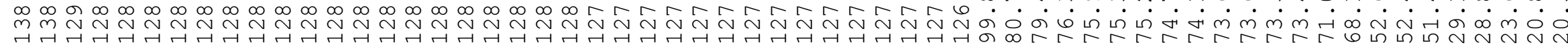

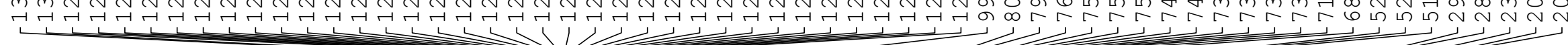

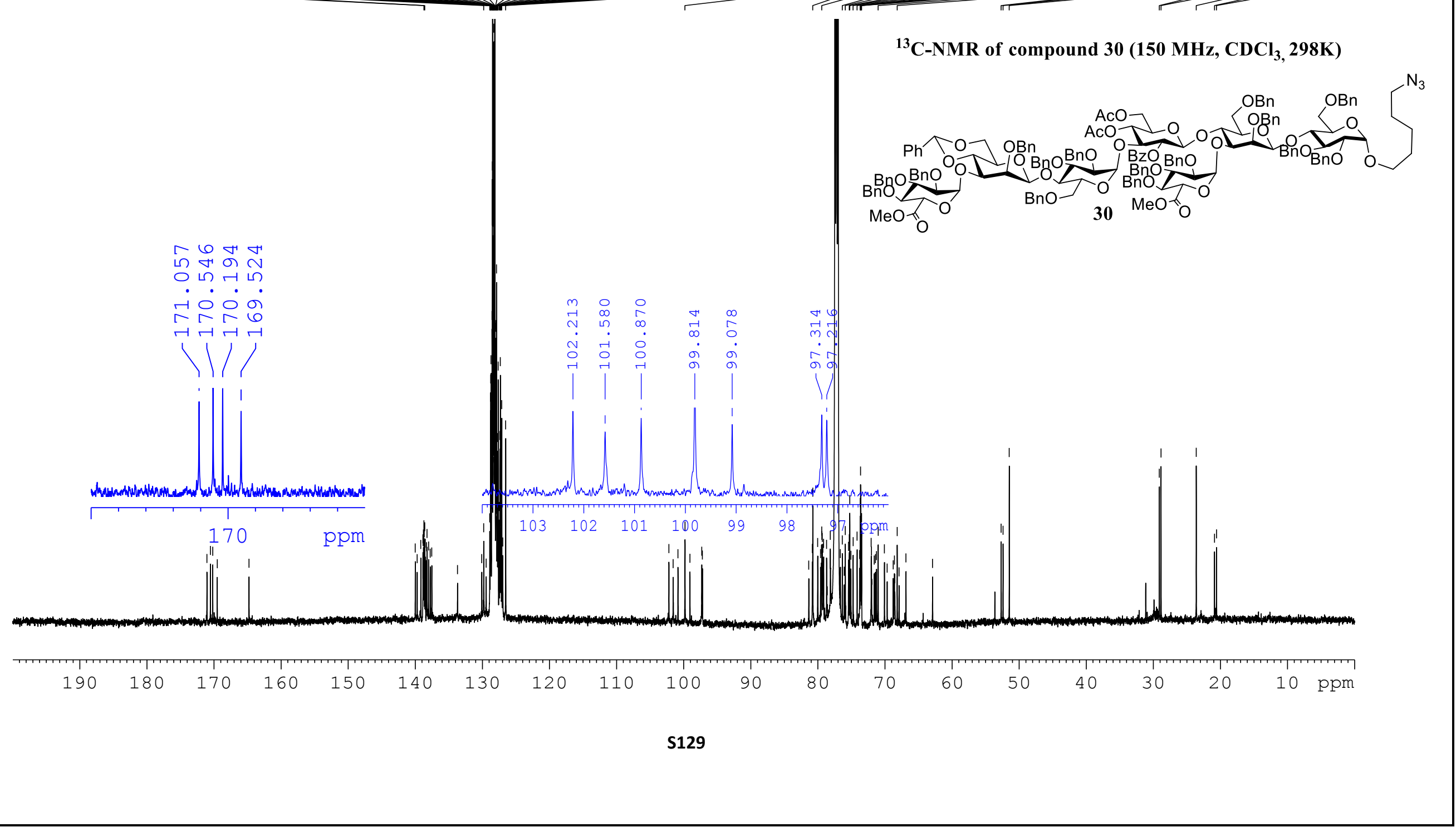


\#

Hनh

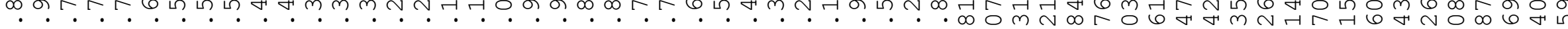

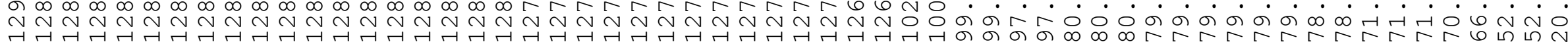

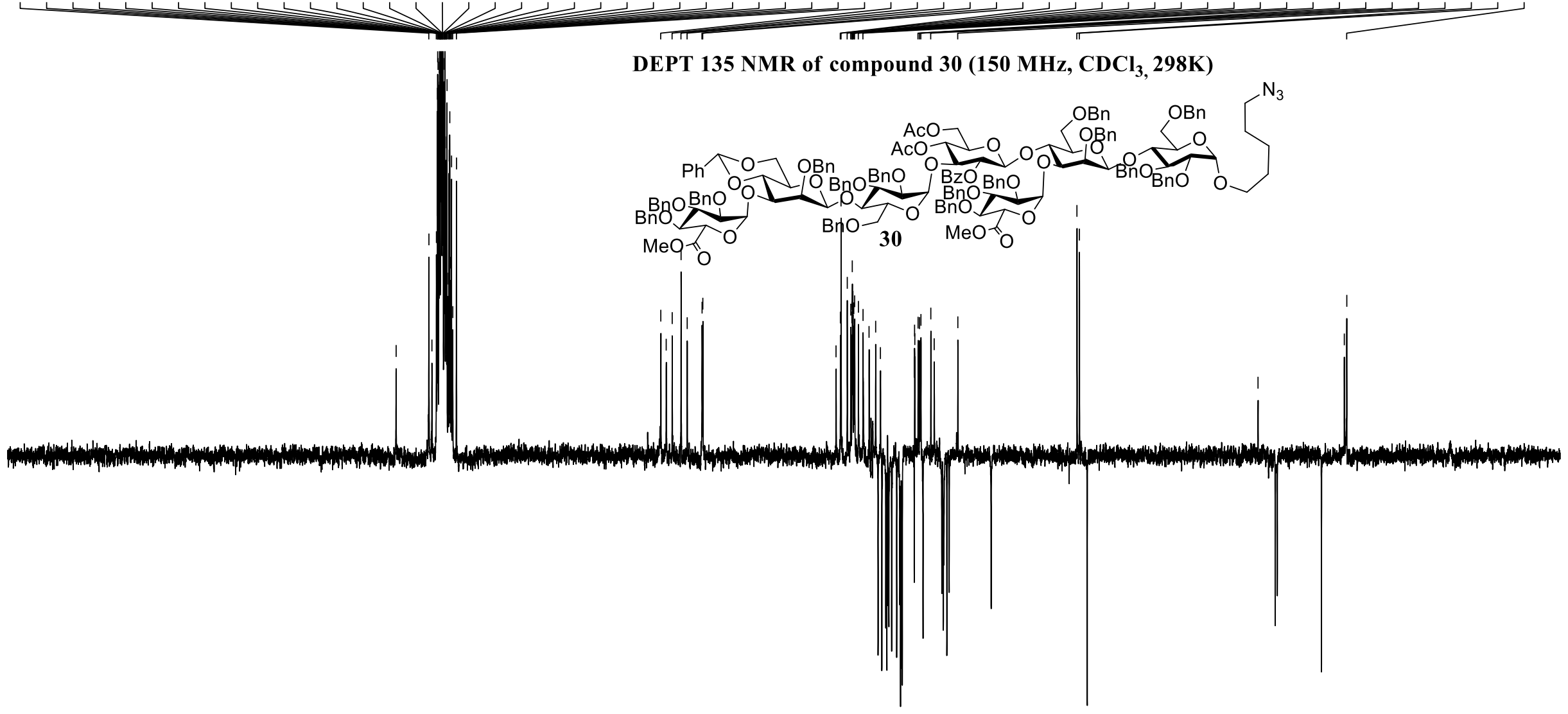

S130 


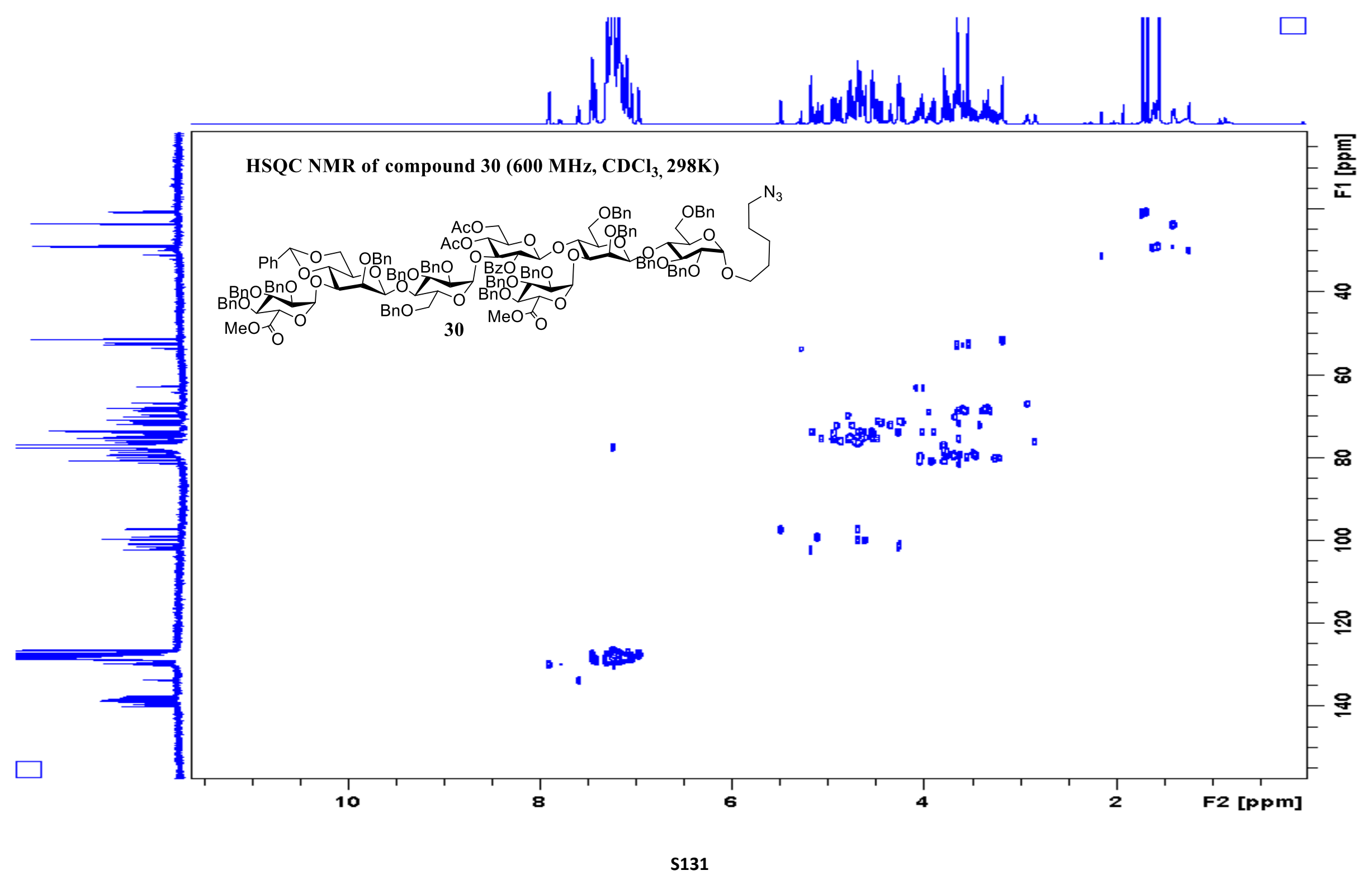




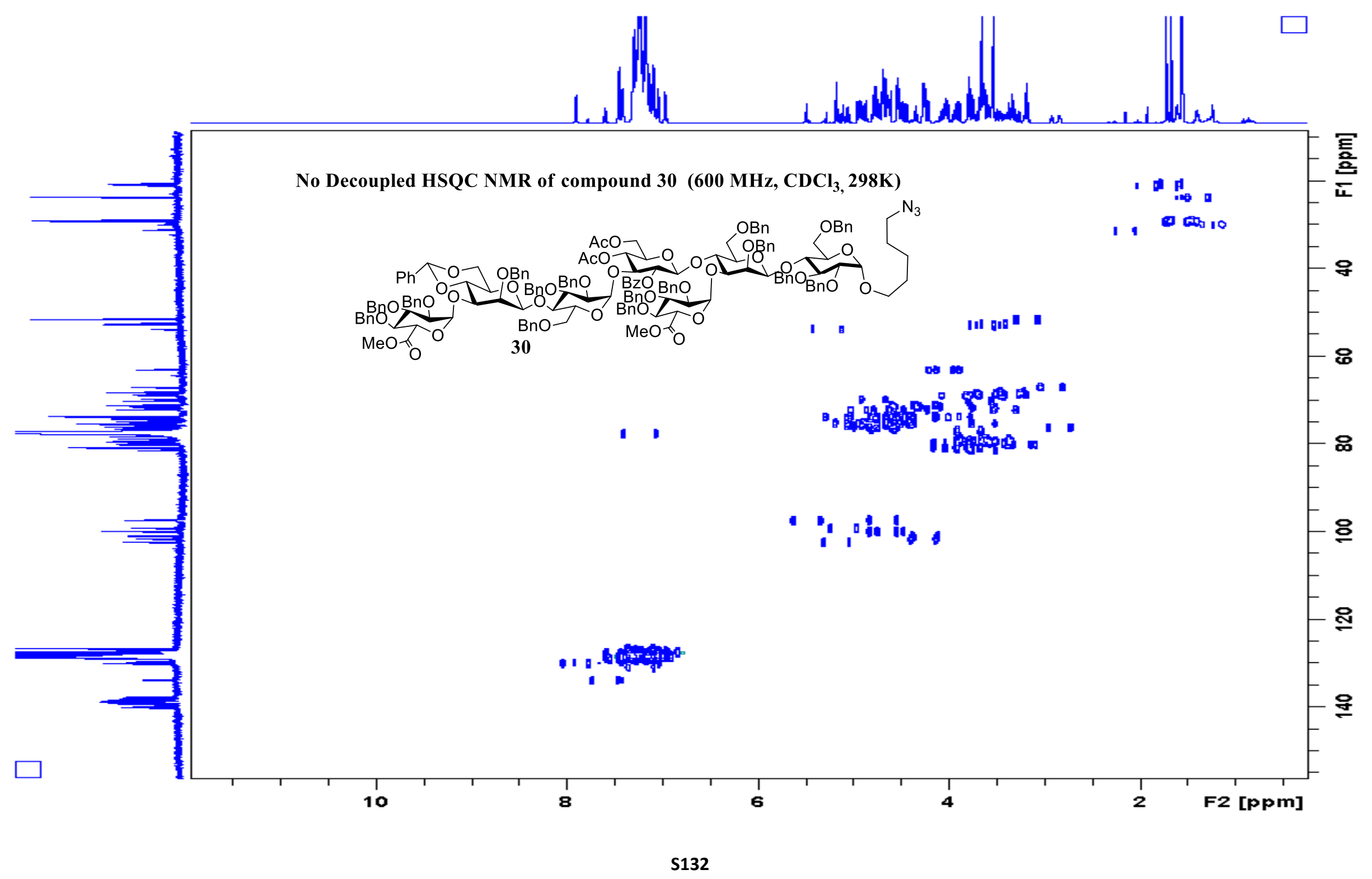




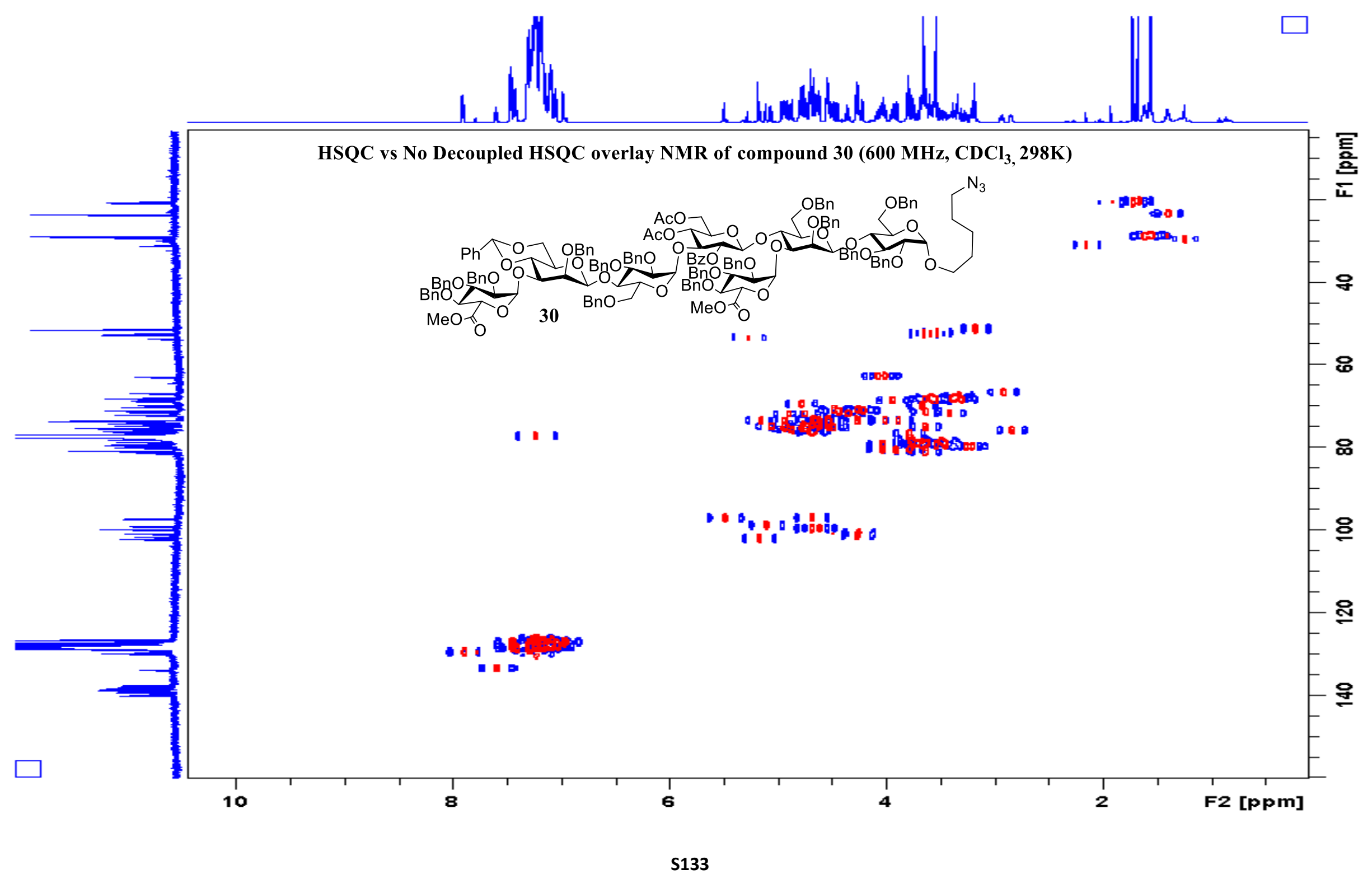




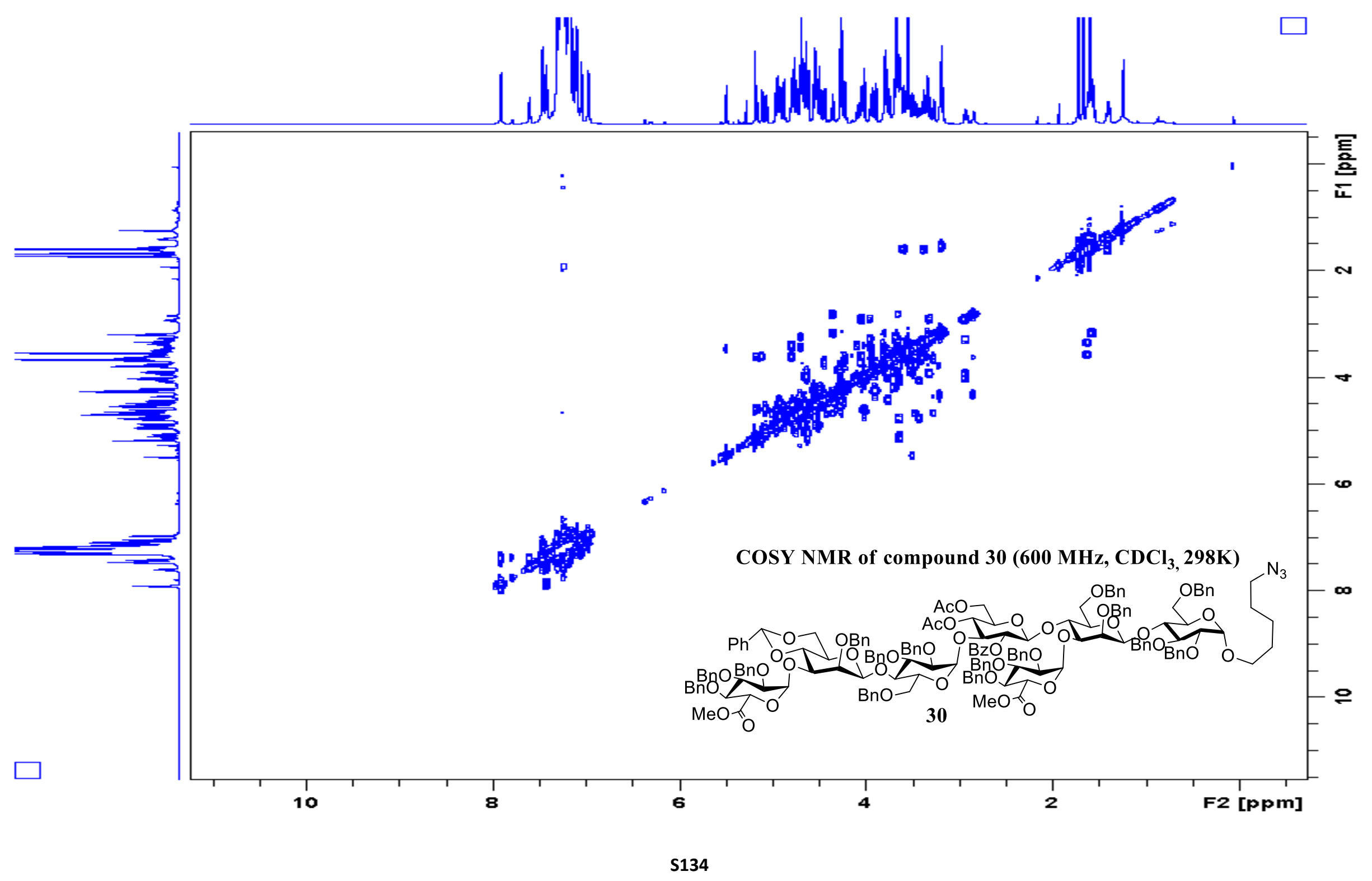




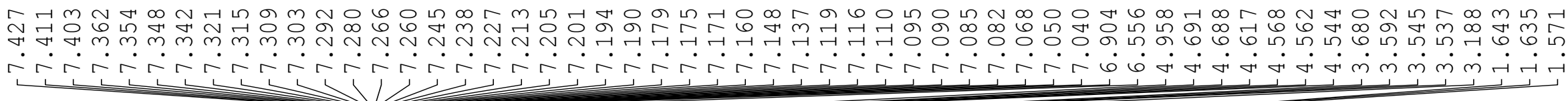

${ }^{1} \mathrm{H}-\mathrm{NMR}$ of compound 31 (600 $\left.\mathrm{MHz}, \mathrm{CDCl}_{3}, 298 \mathrm{~K}\right)$
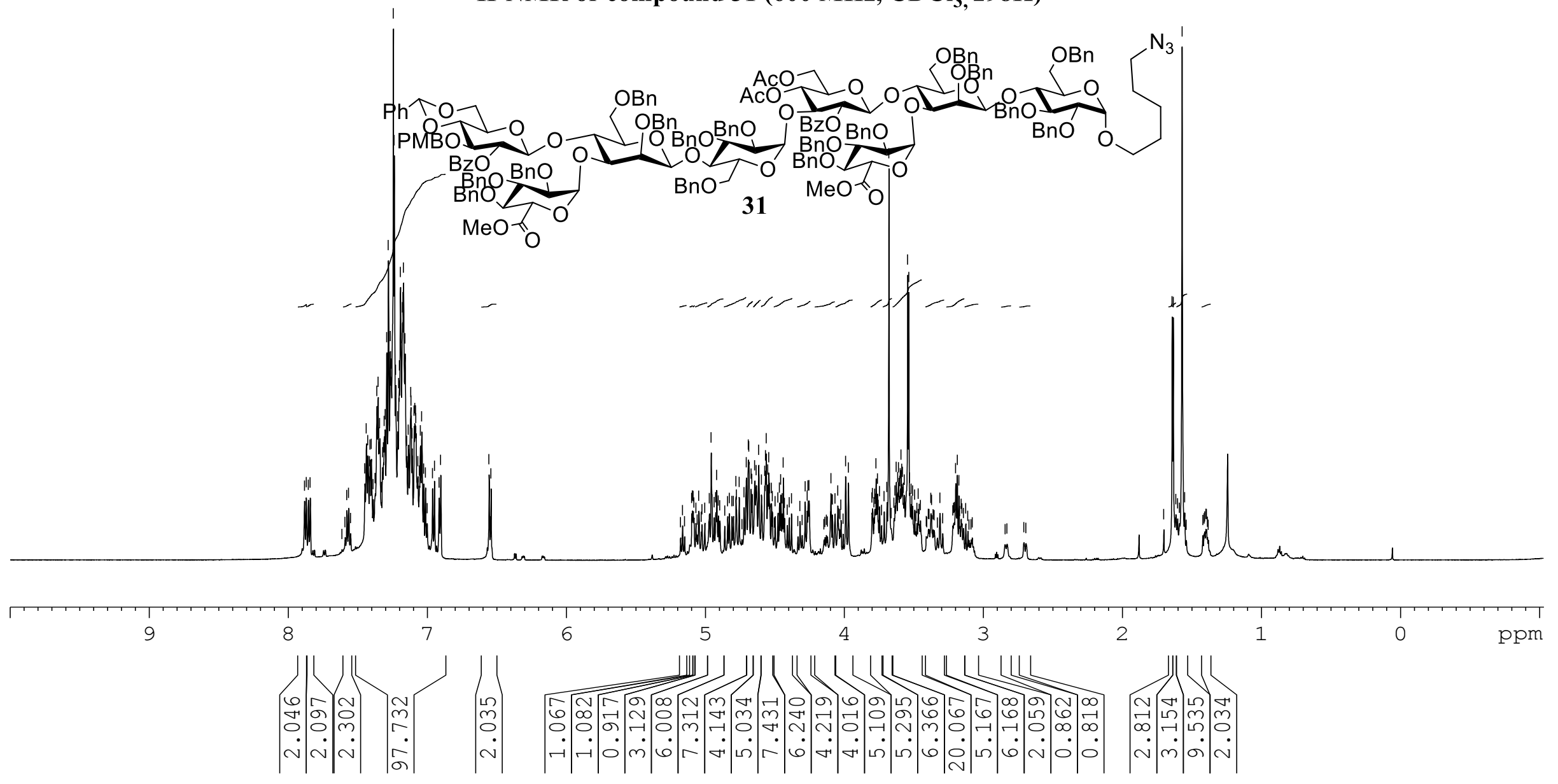

S135 


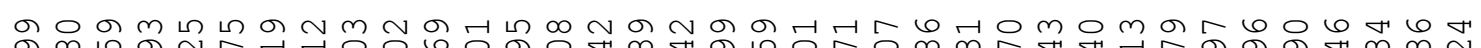

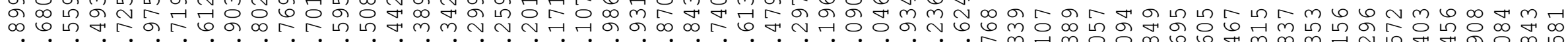

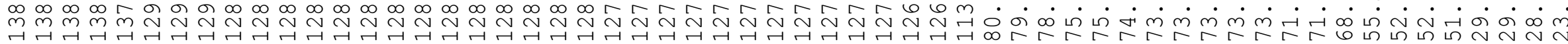

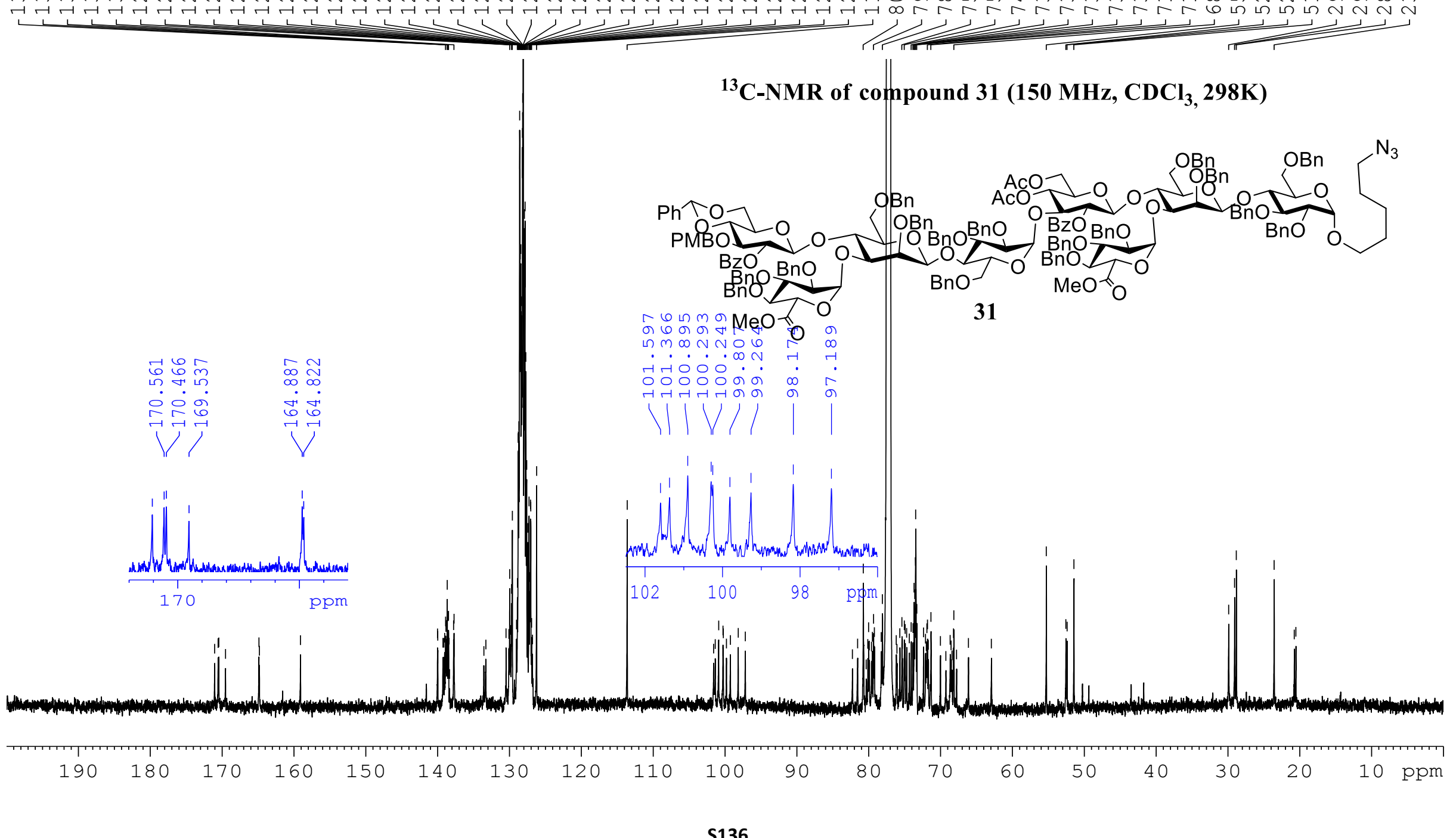

S136 


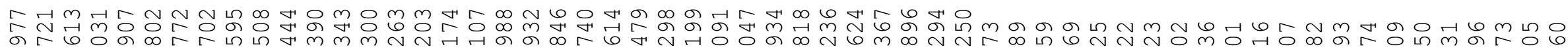

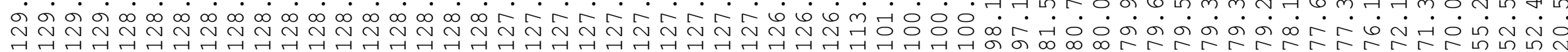

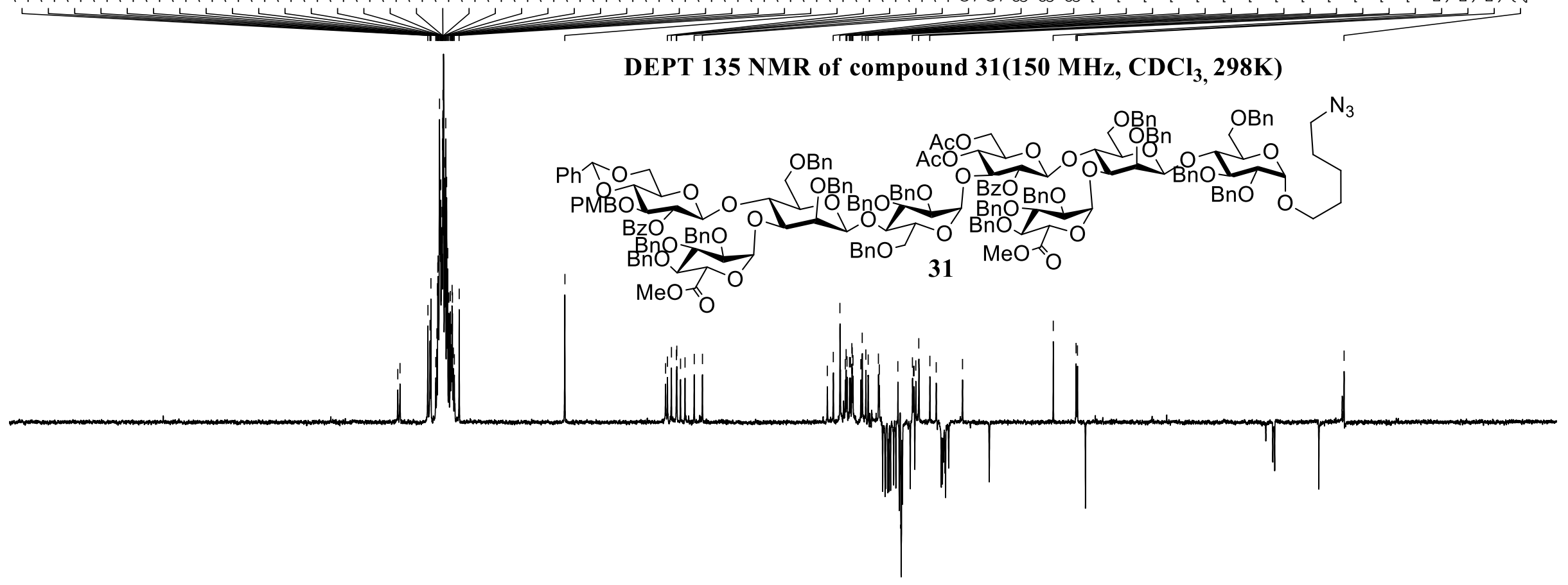

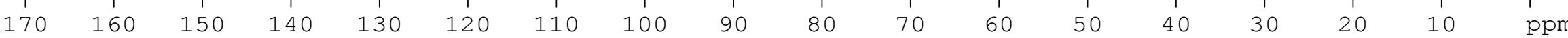




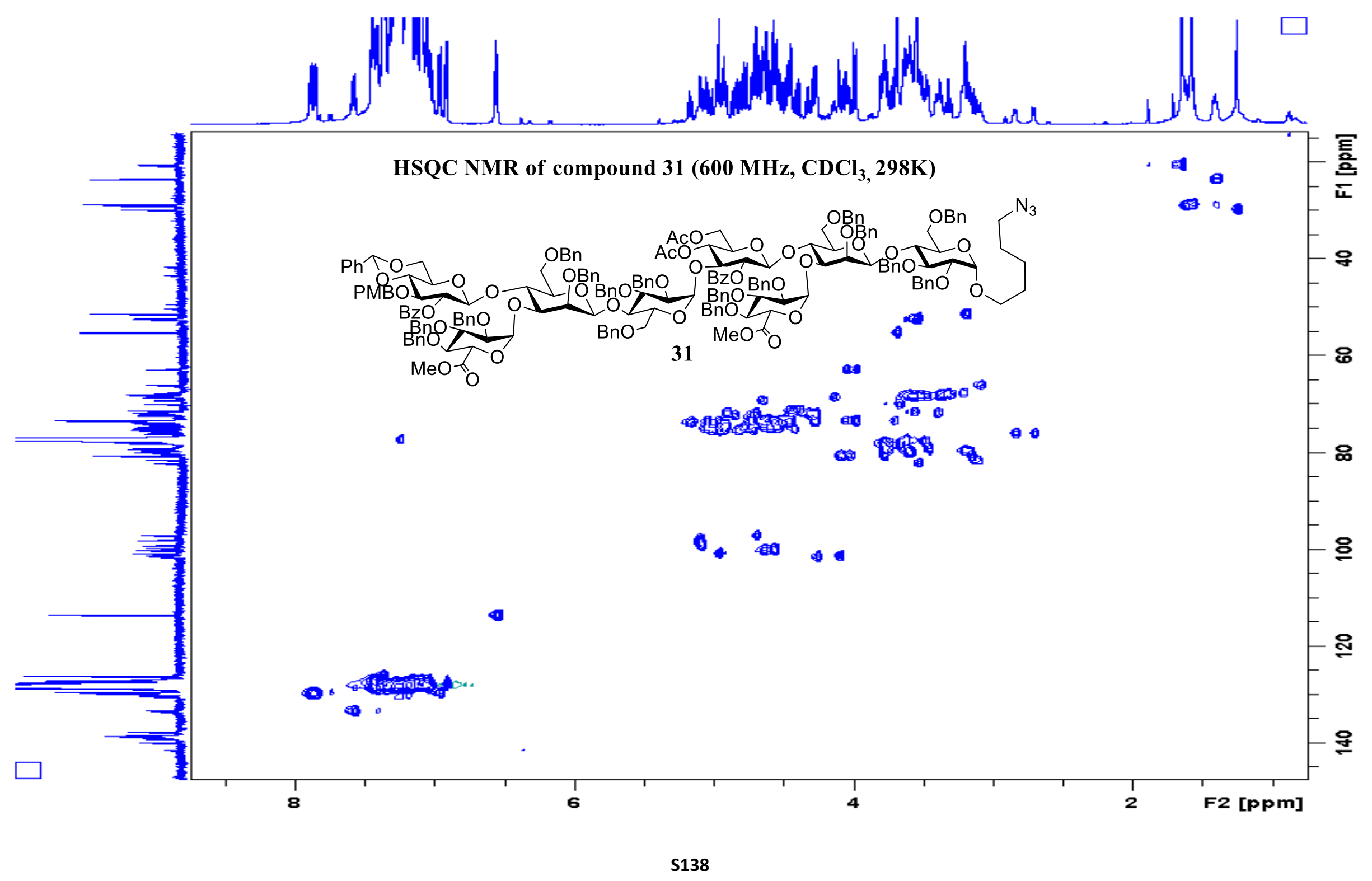




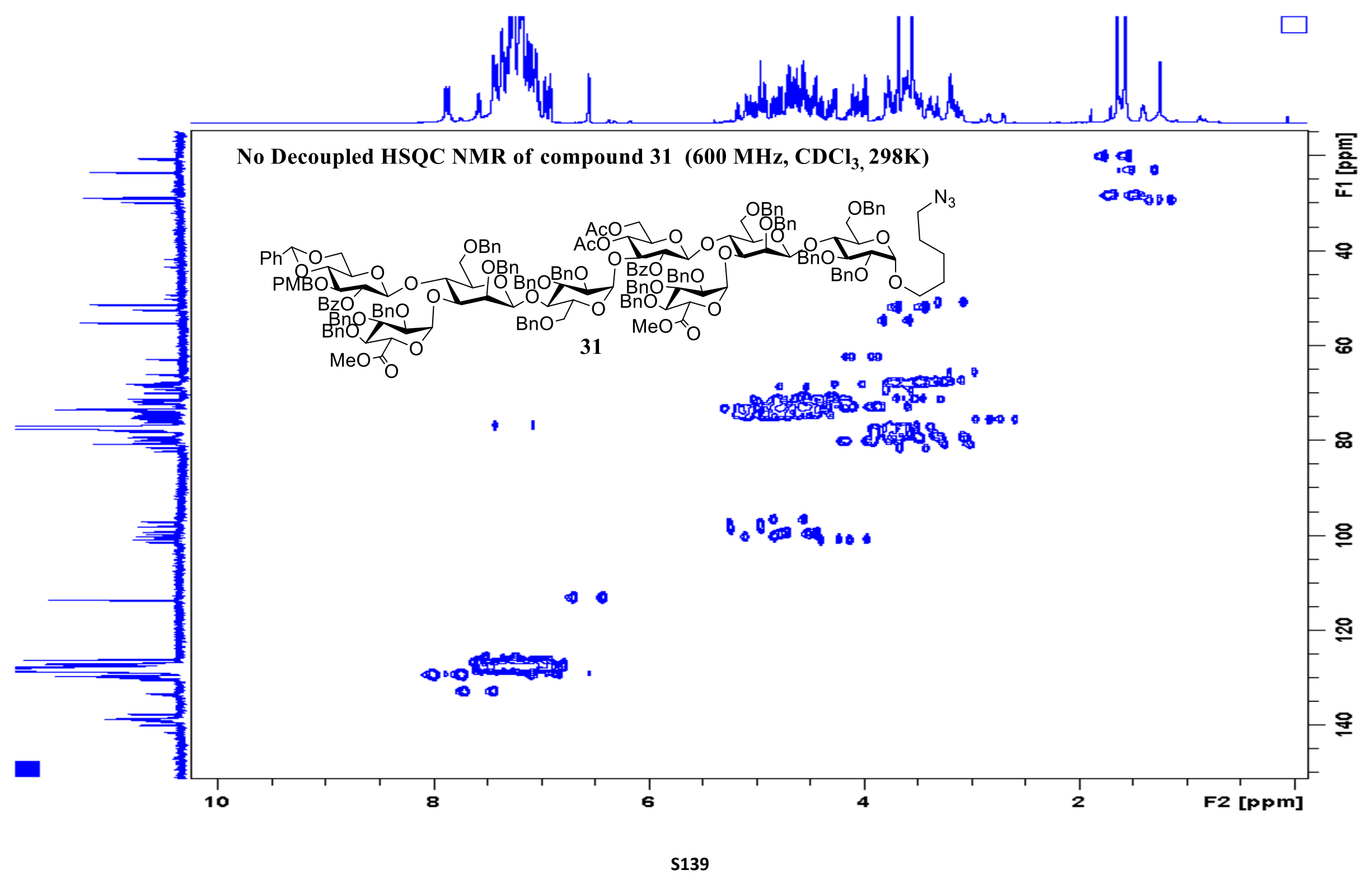




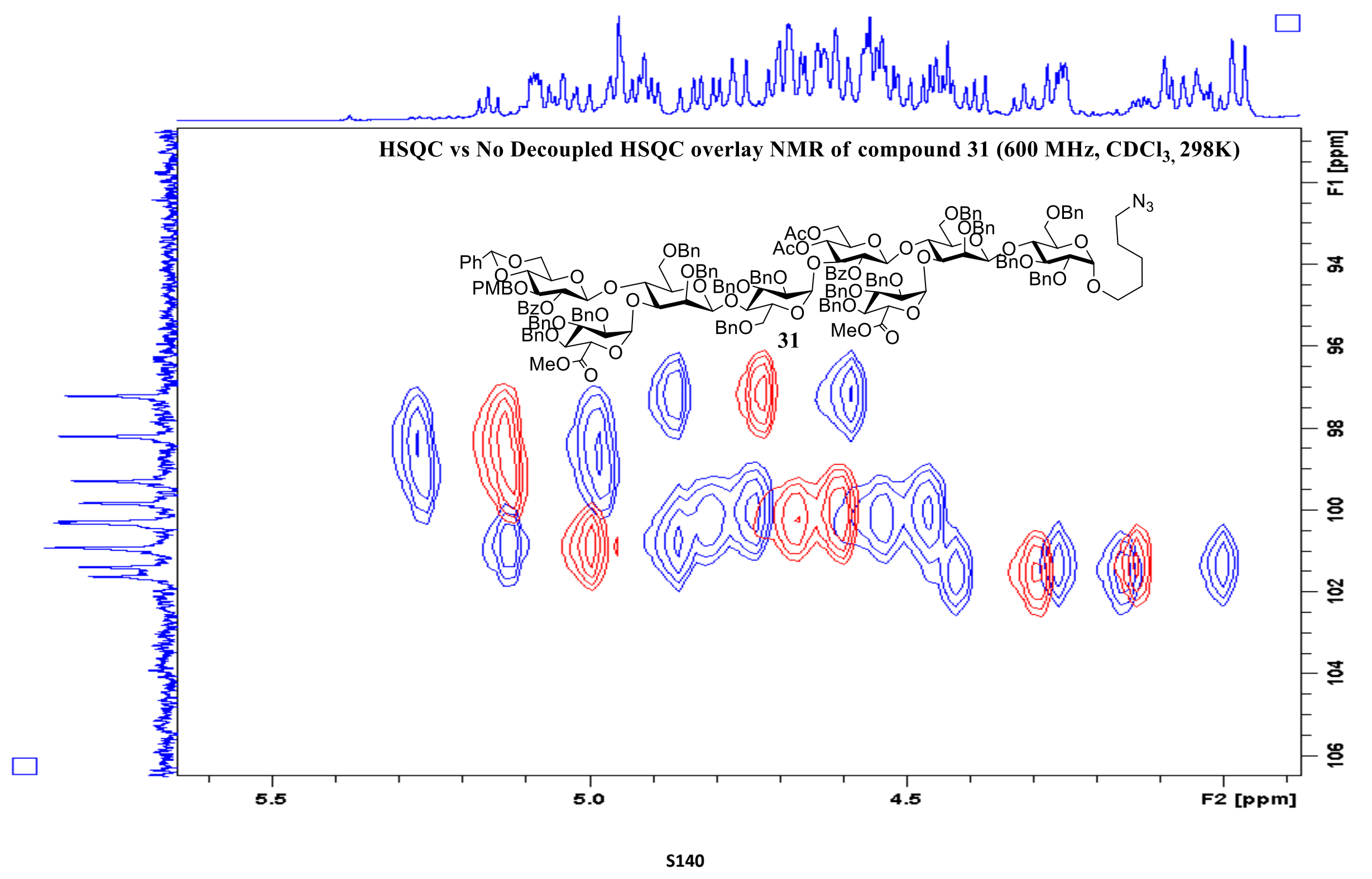




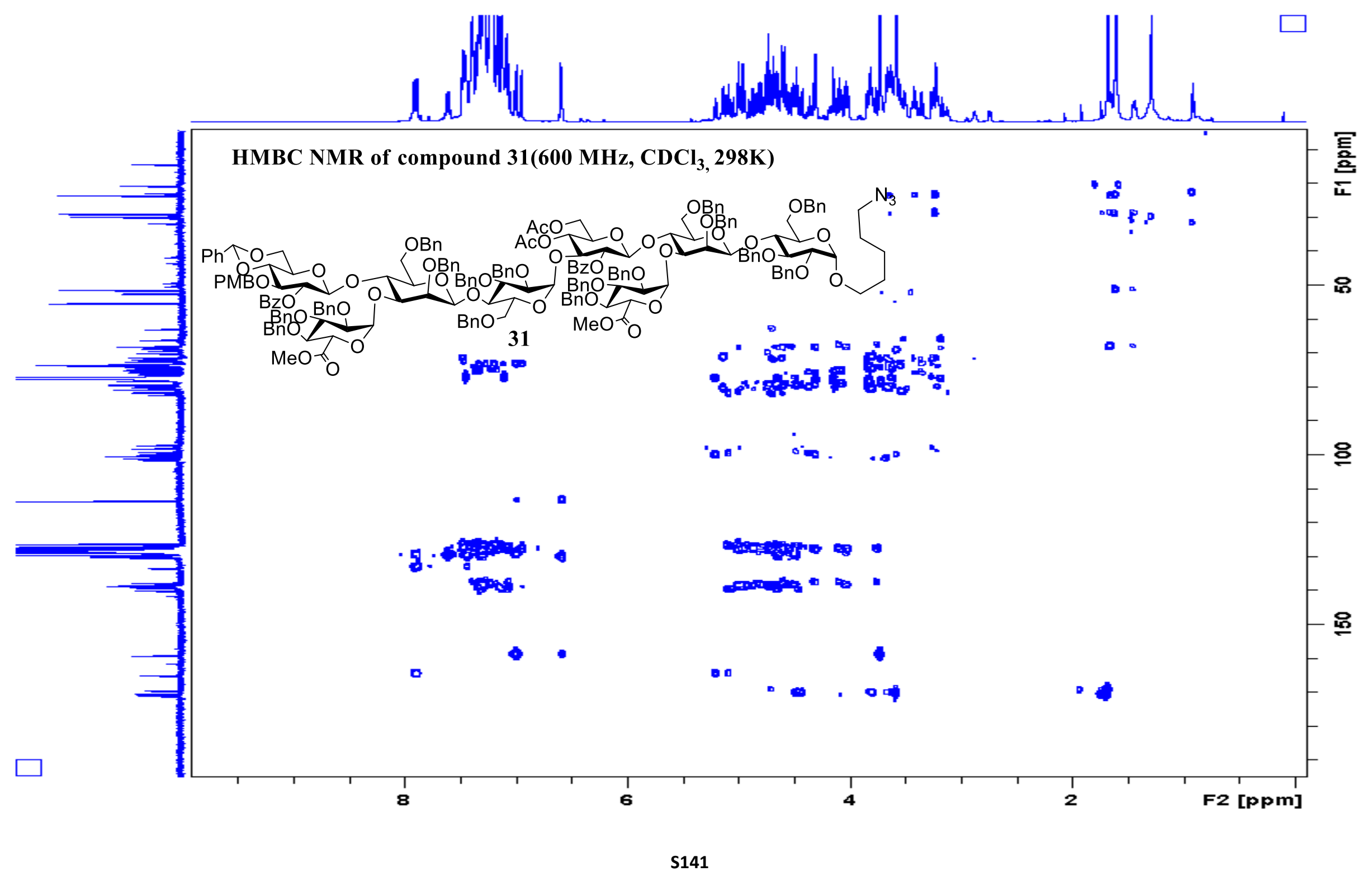




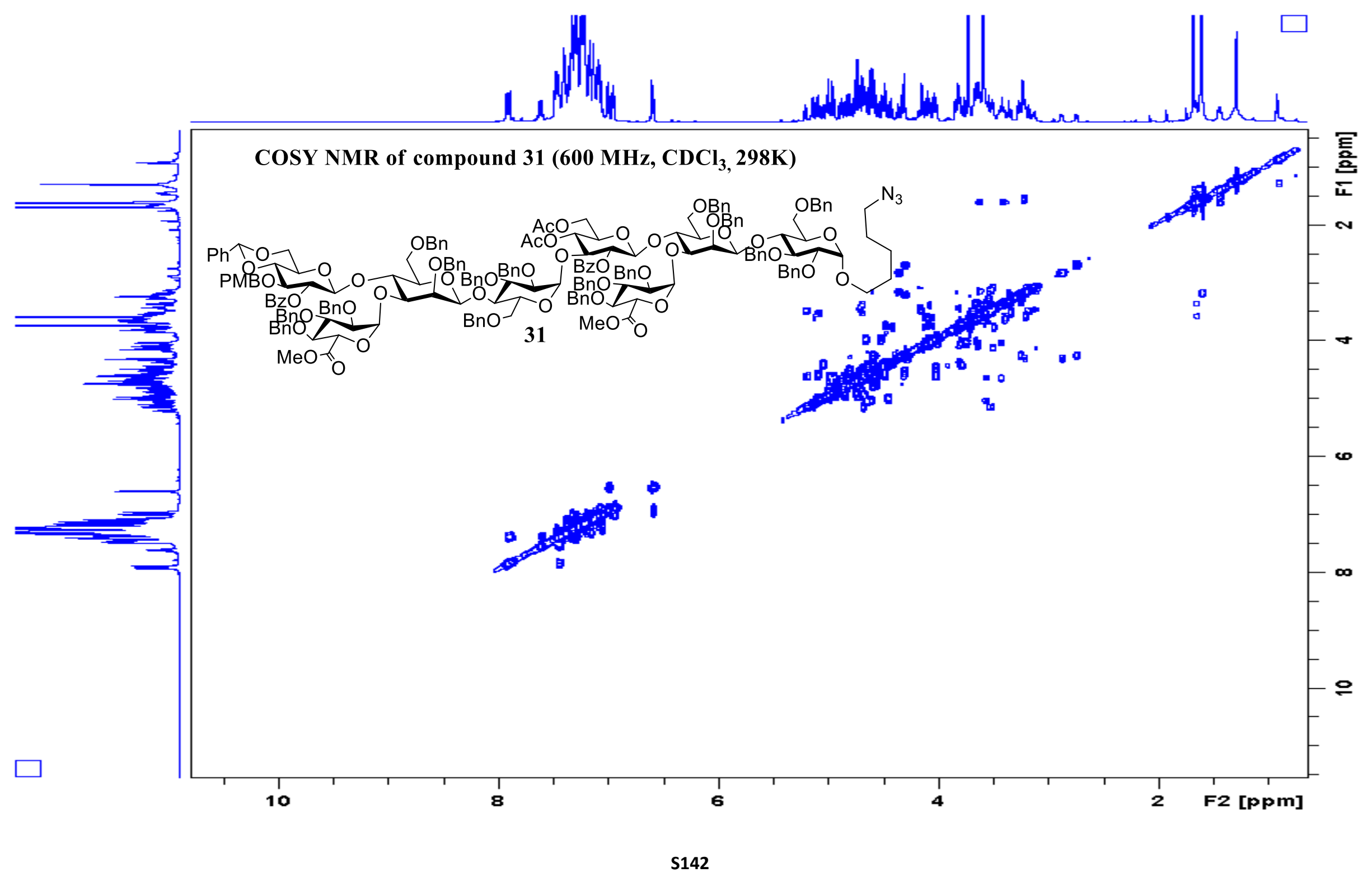




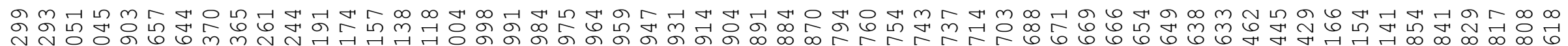

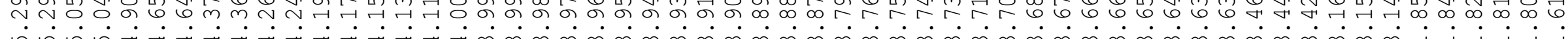
ம்

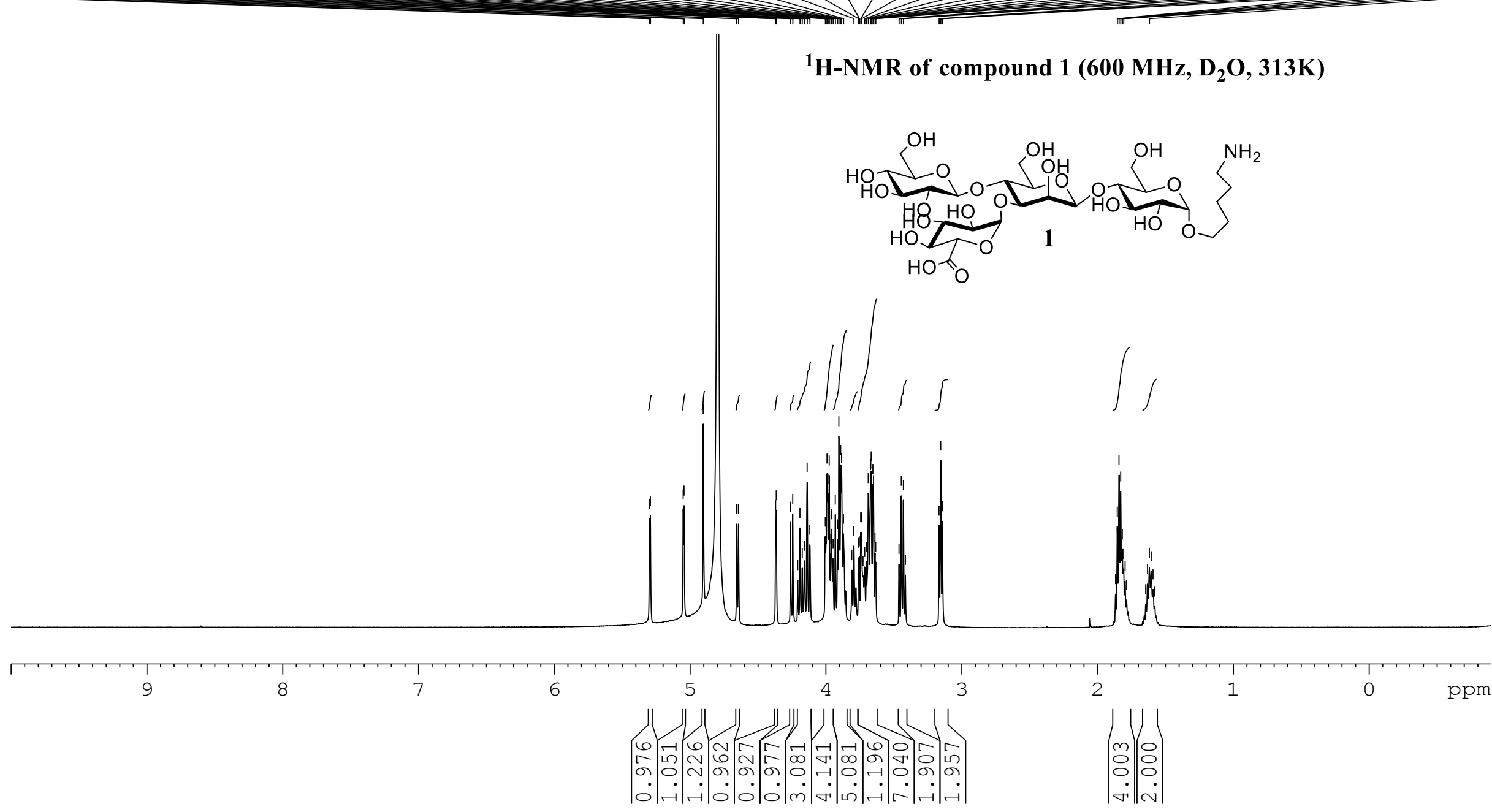




\section{${ }^{13} \mathrm{C}-\mathrm{NMR}$ of compound $1\left(150 \mathrm{MHz}, \mathrm{D}_{2} \mathrm{O}, 313 \mathrm{~K}\right)$}

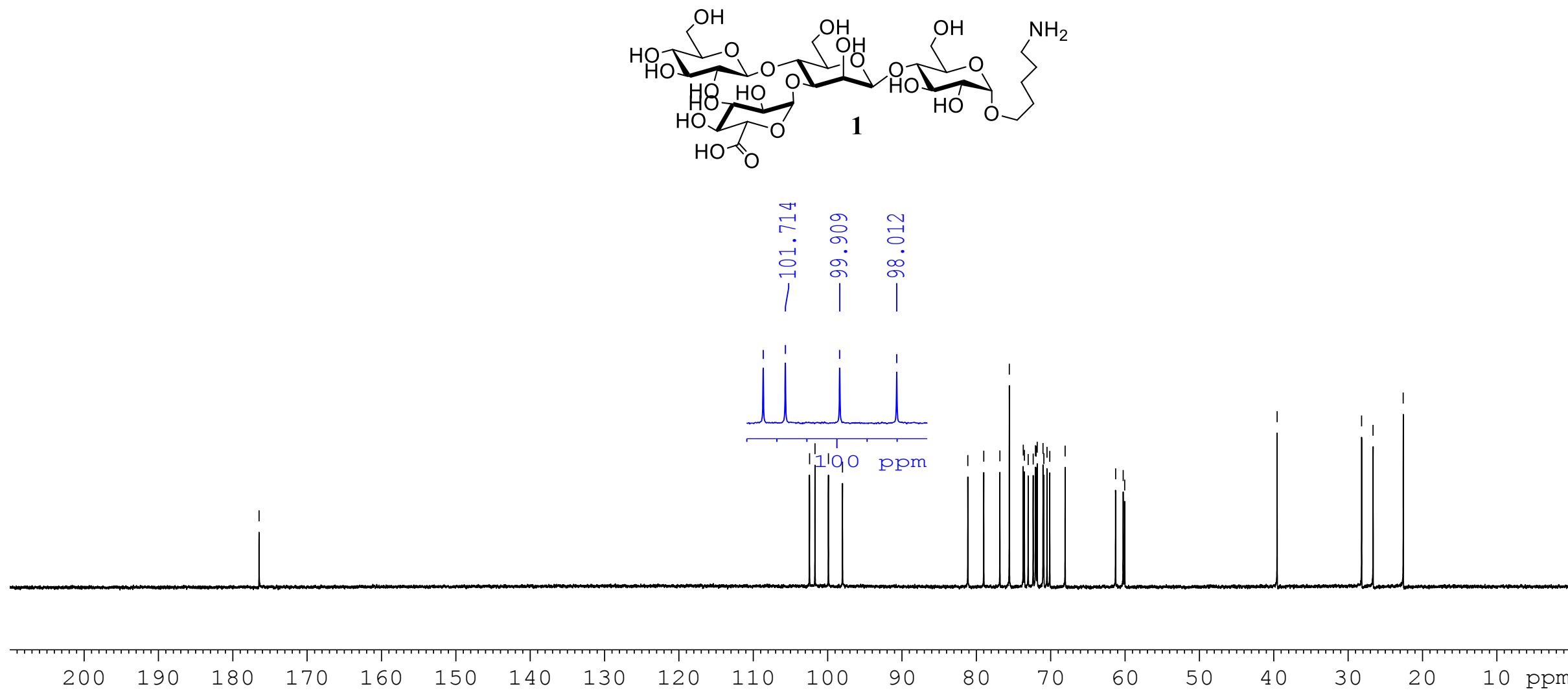




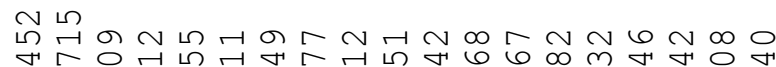

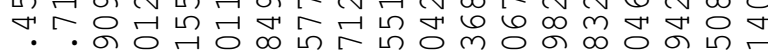

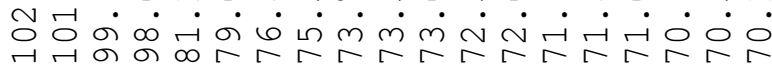

DEPT 135 NMR of compound $1\left(150 \mathrm{MHz}, \mathrm{D}_{2} \mathrm{O}, 313 \mathrm{~K}\right)$

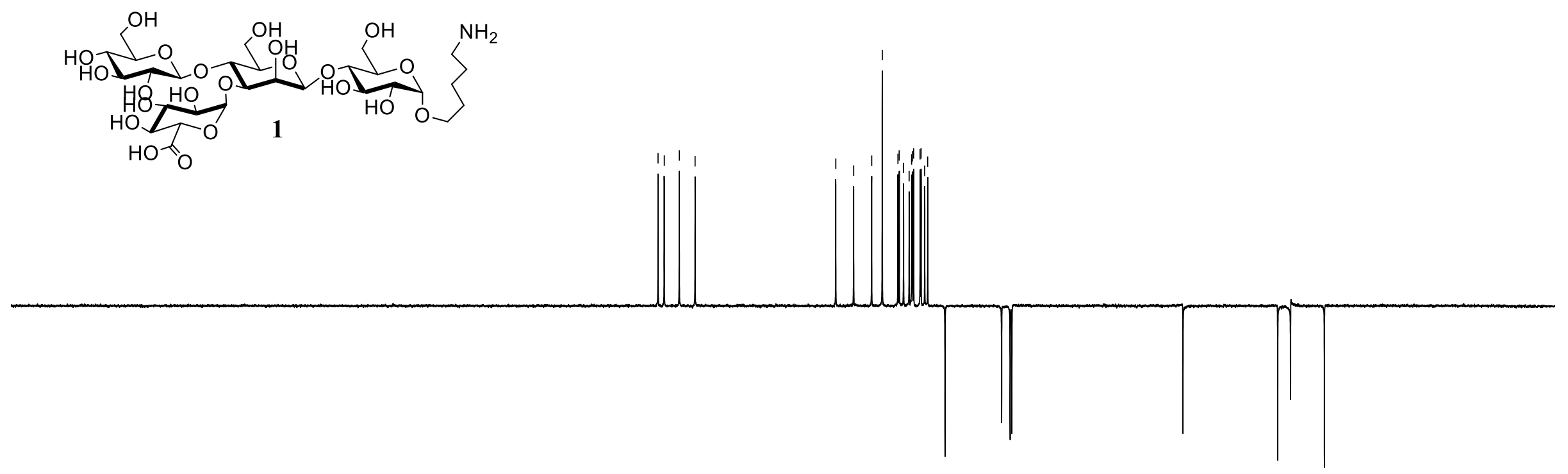

$\begin{array}{lllllllllllllllllll}170 & 160 & 150 & 140 & 130 & 120 & 110 & 100 & 90 & 80 & 70 & 60 & 50 & 40 & 30 & 20 & 10 & \text { ppm }\end{array}$




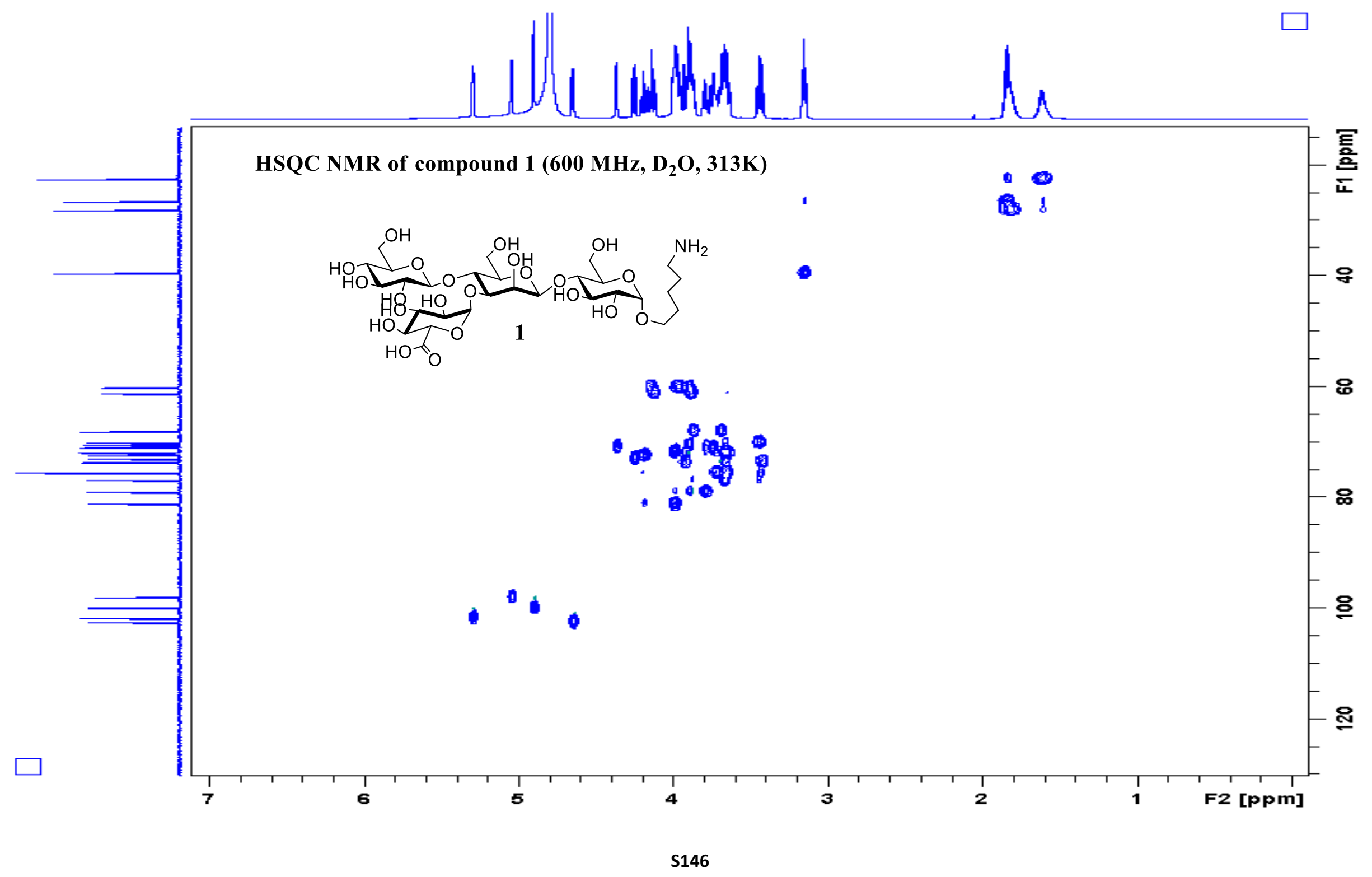




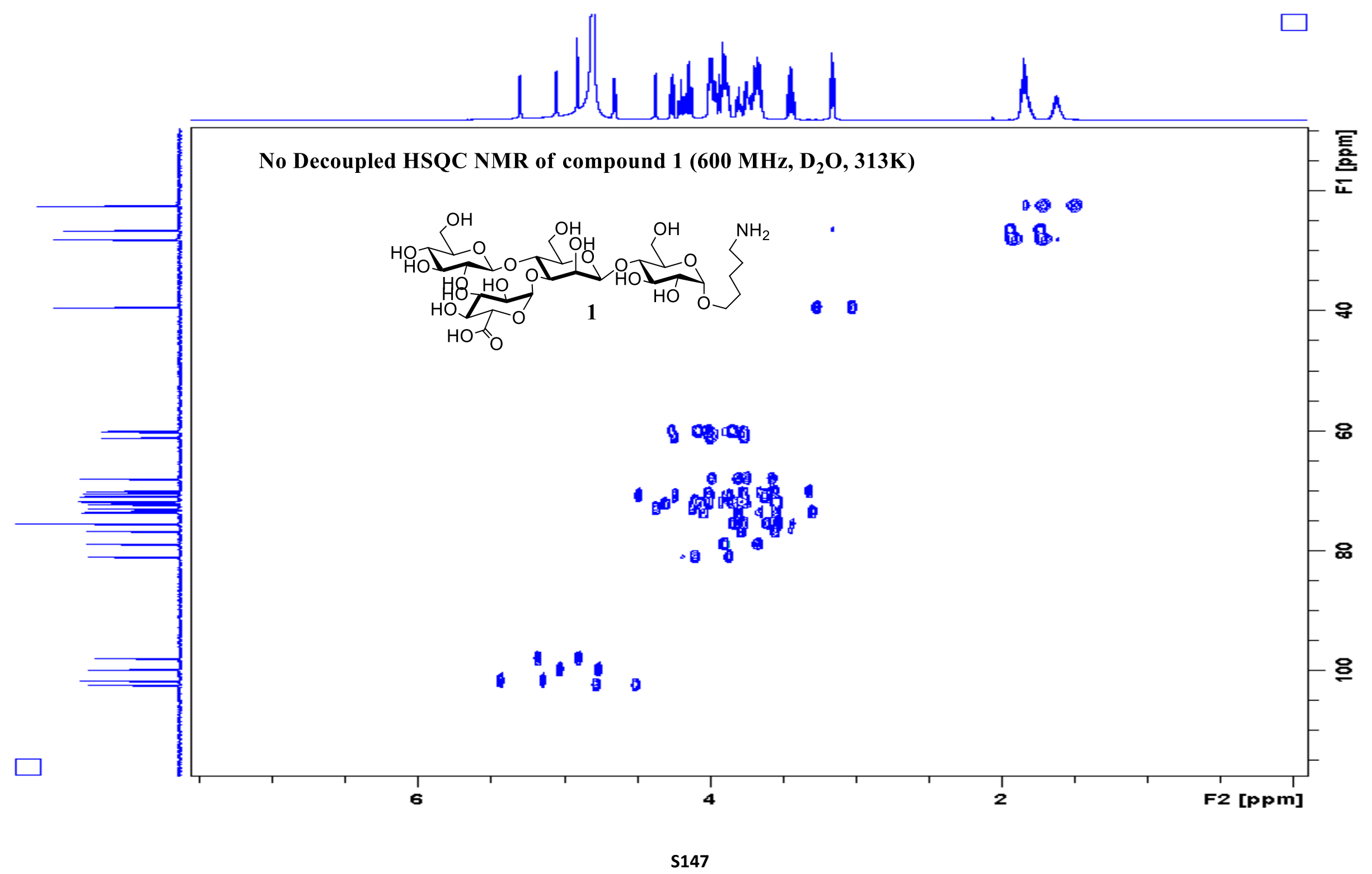




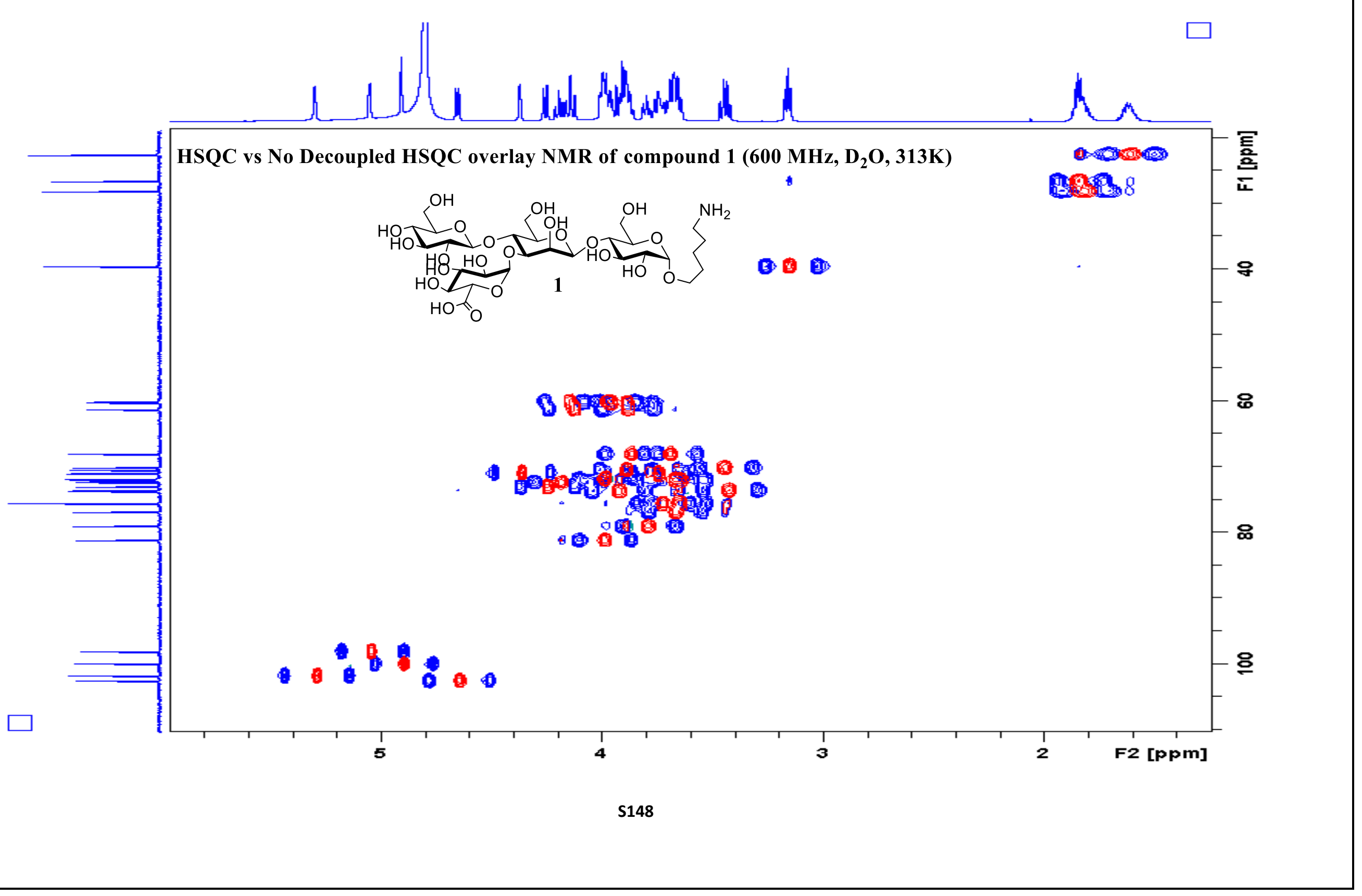




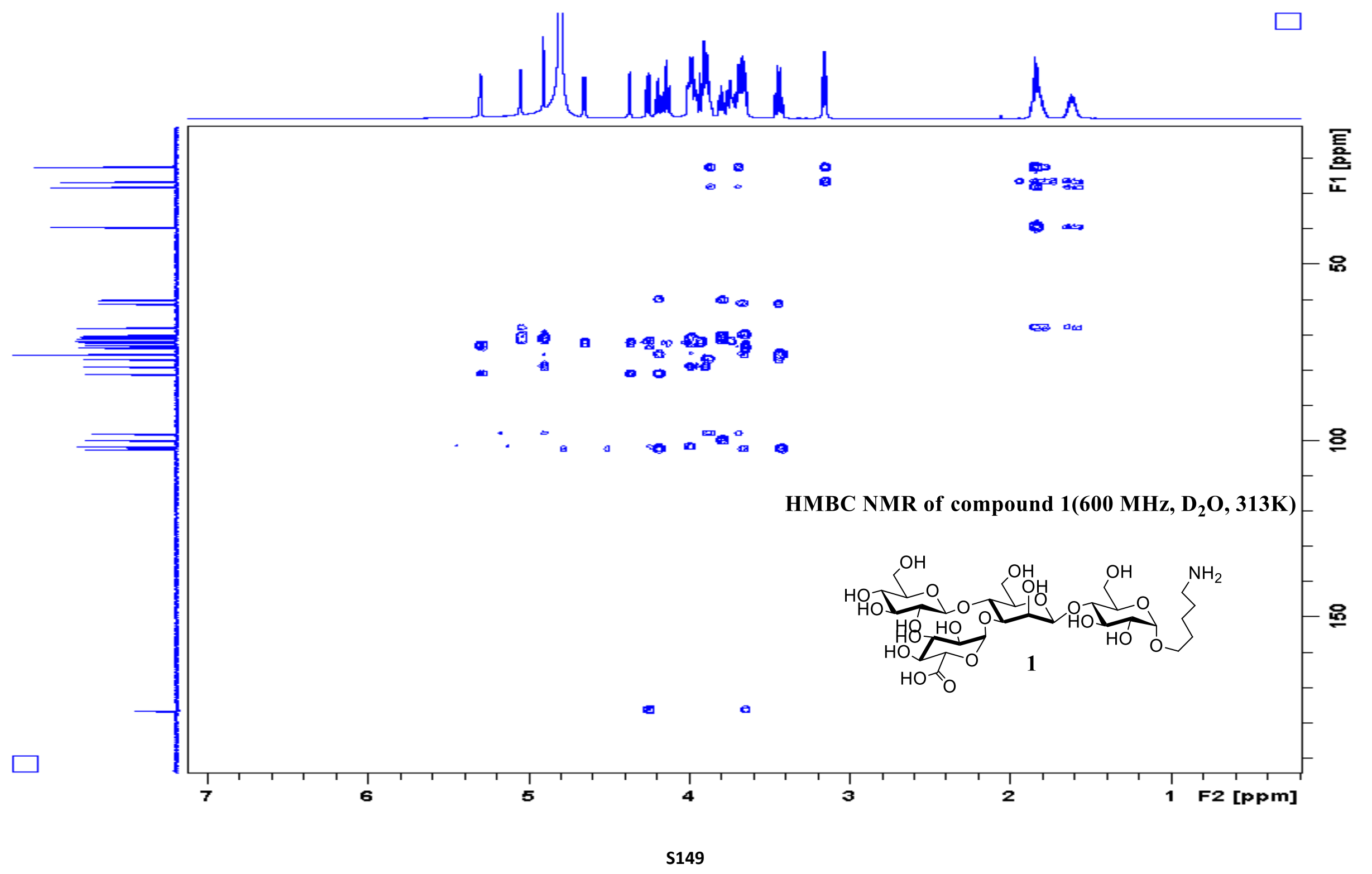




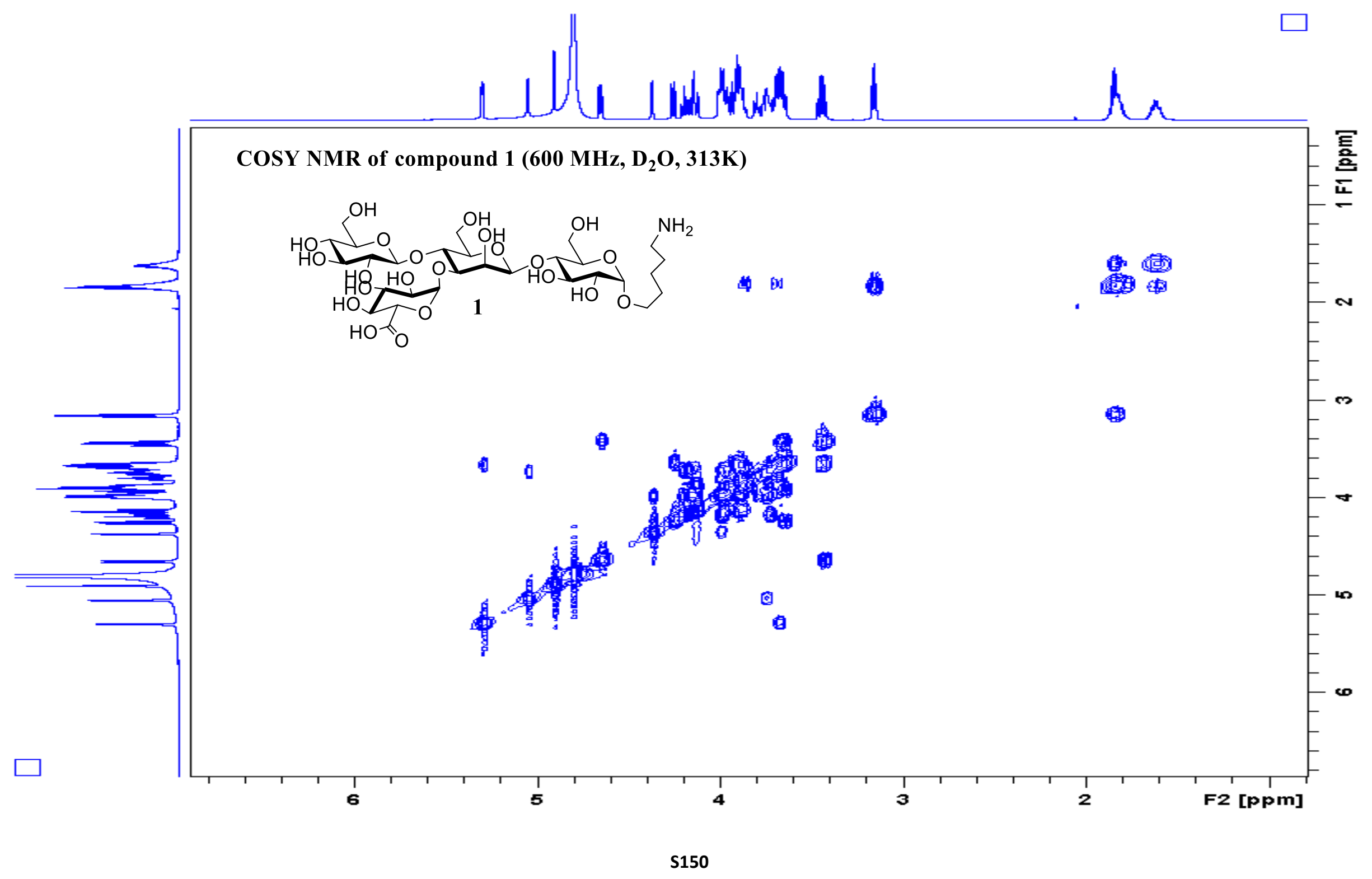




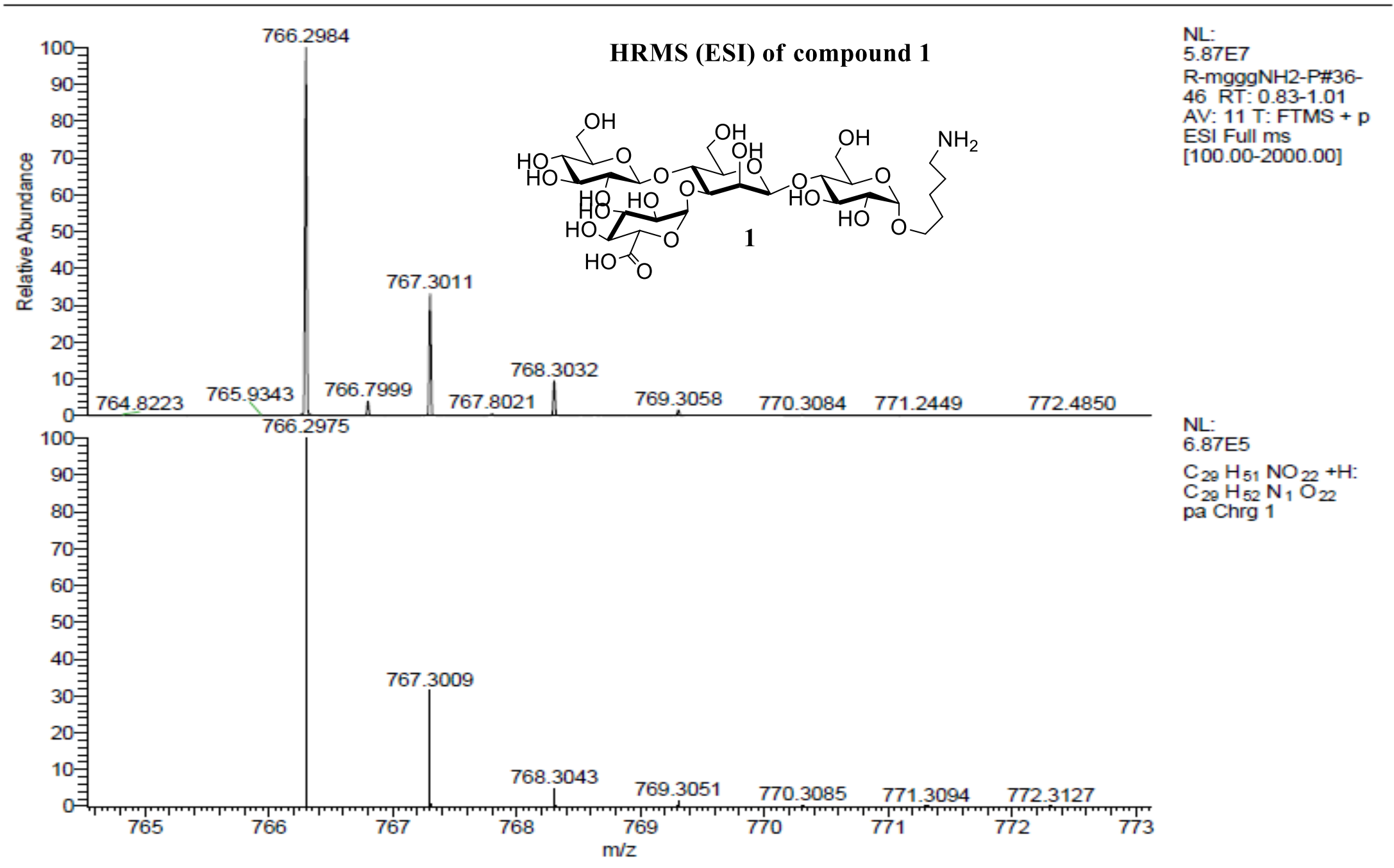




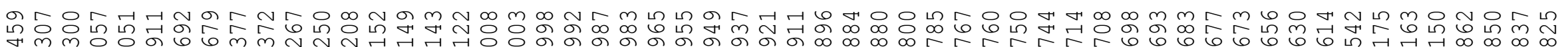
.

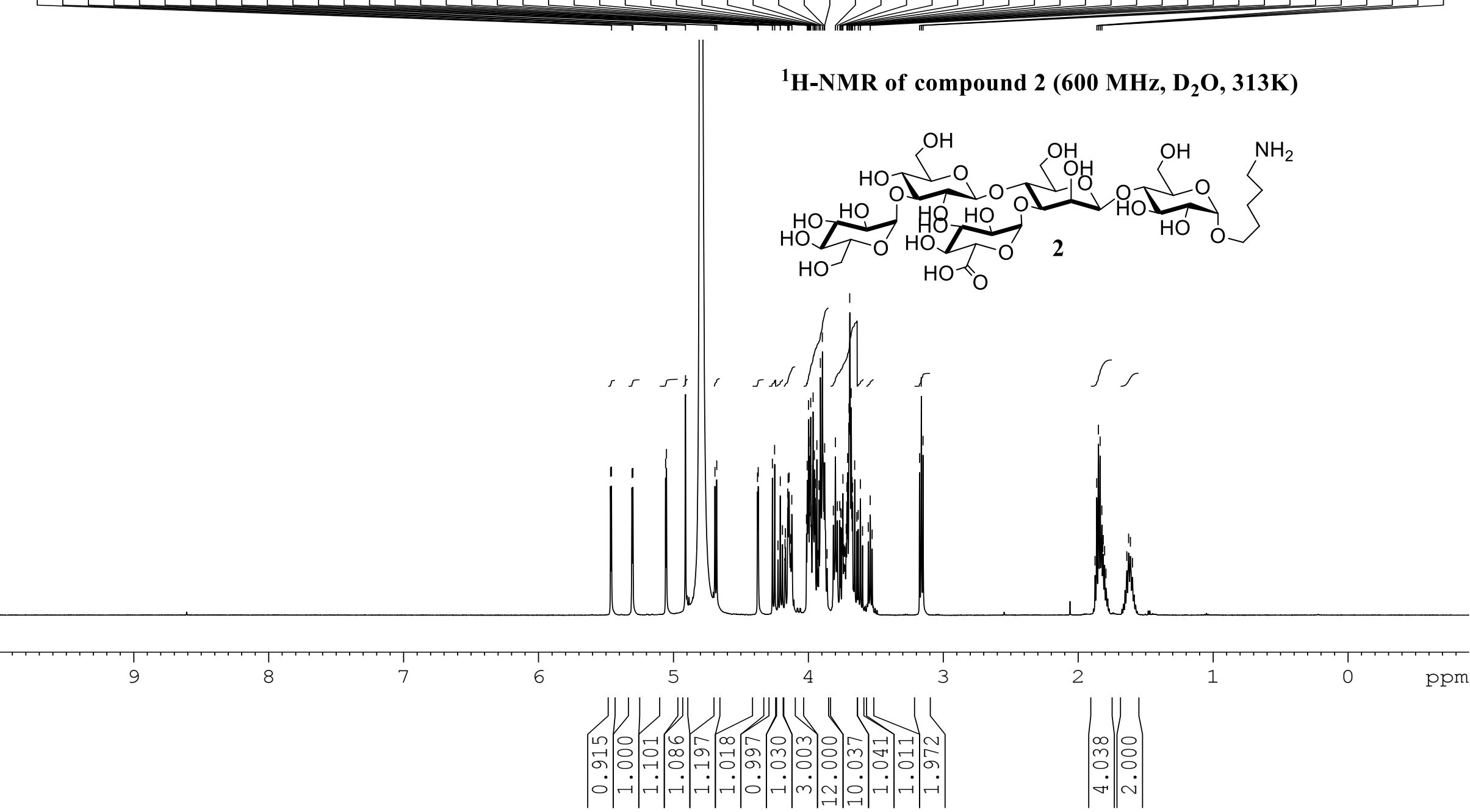




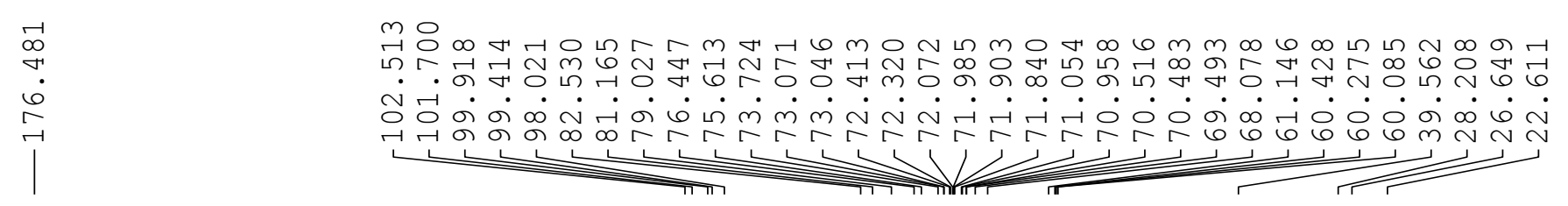

${ }^{13} \mathrm{C}-\mathrm{NMR}$ of compound $2\left(150 \mathrm{MHz}, \mathrm{D}_{2} \mathrm{O}, 313 \mathrm{~K}\right)$
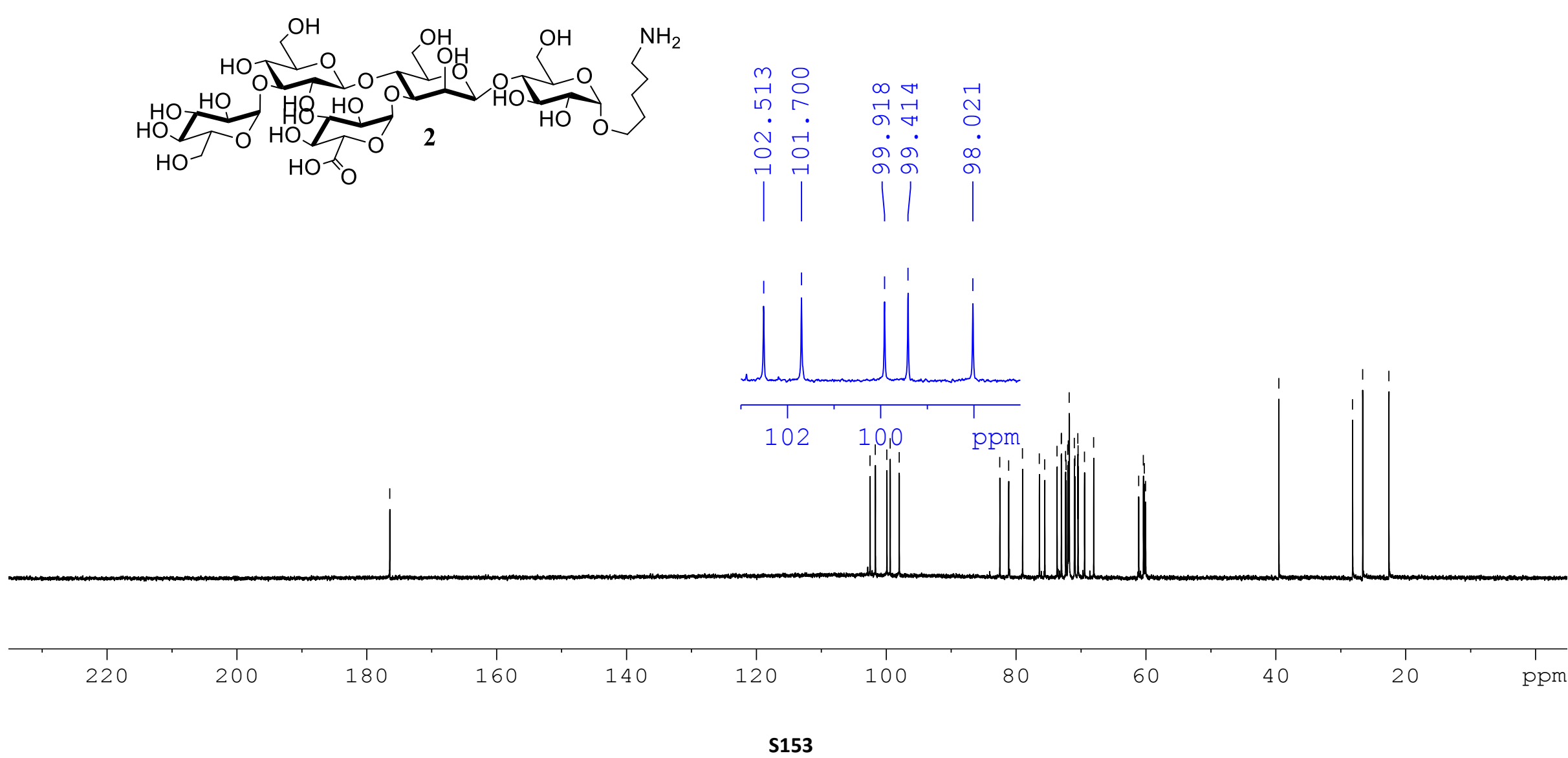


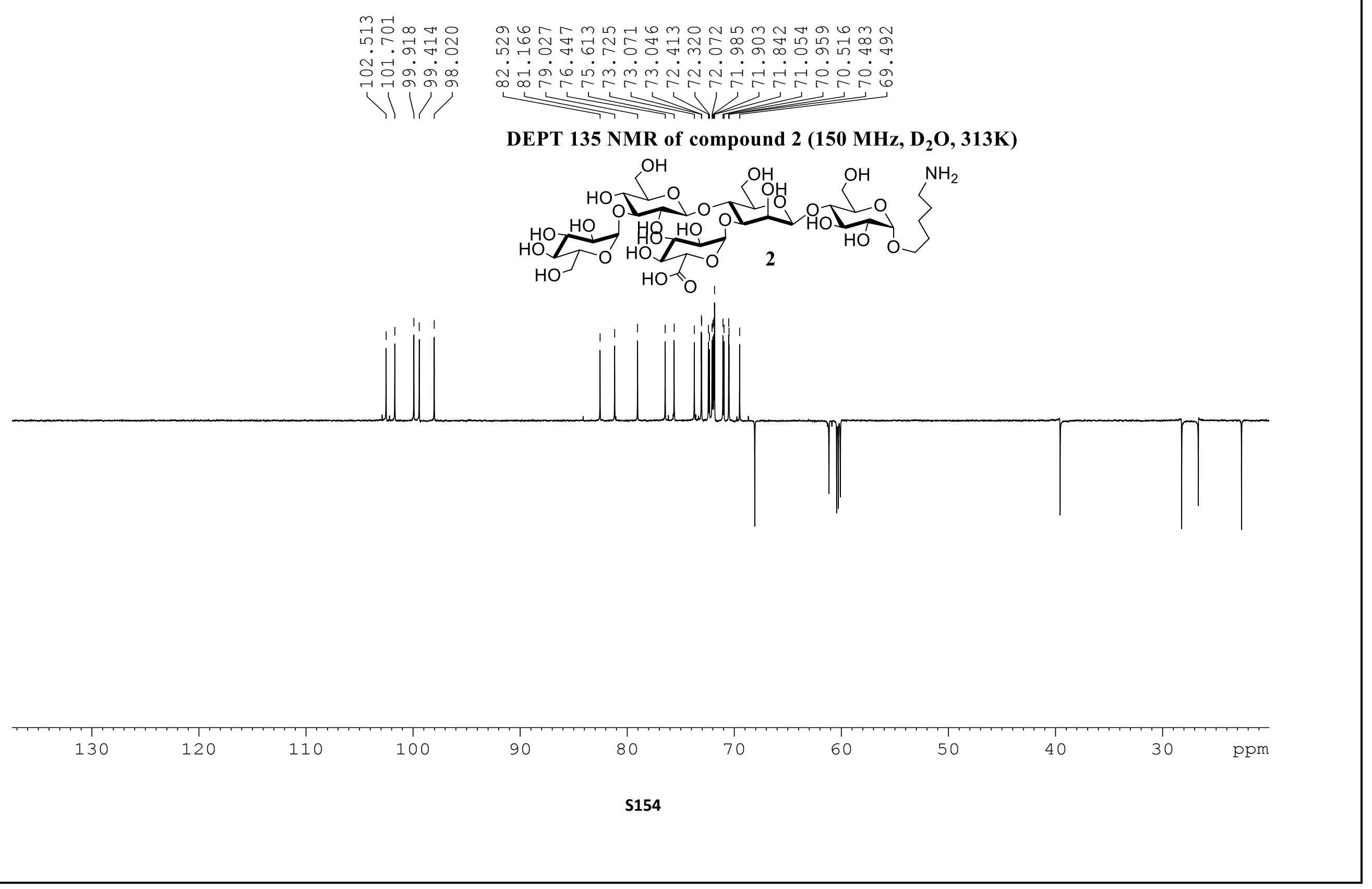



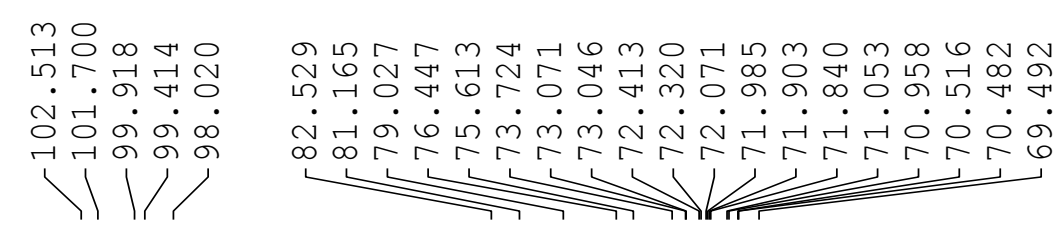

\section{DEPT 90 NMR of compound 2 (150 MHz, $\left.\mathrm{D}_{2} \mathrm{O}, 313 \mathrm{~K}\right)$}
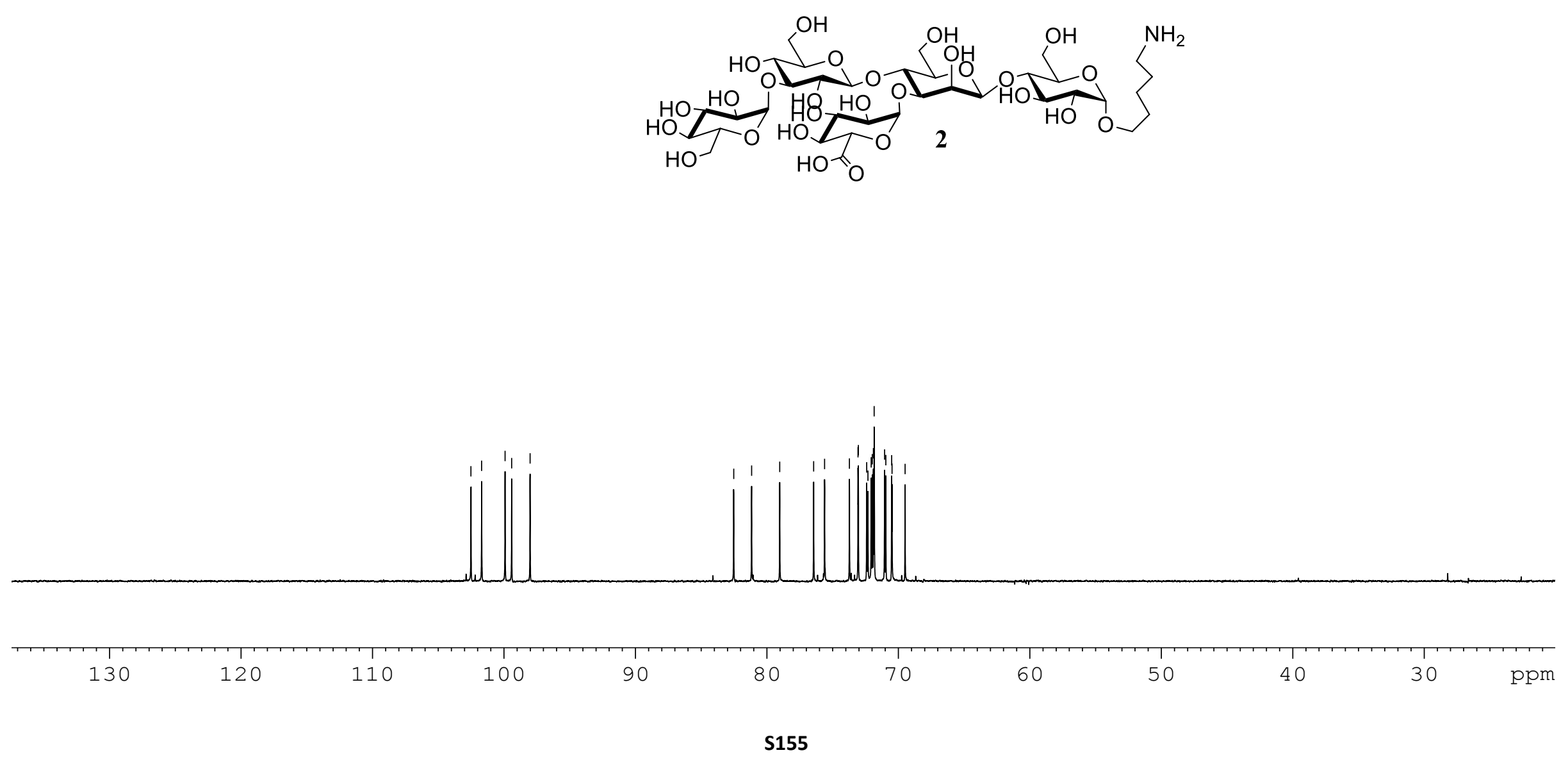


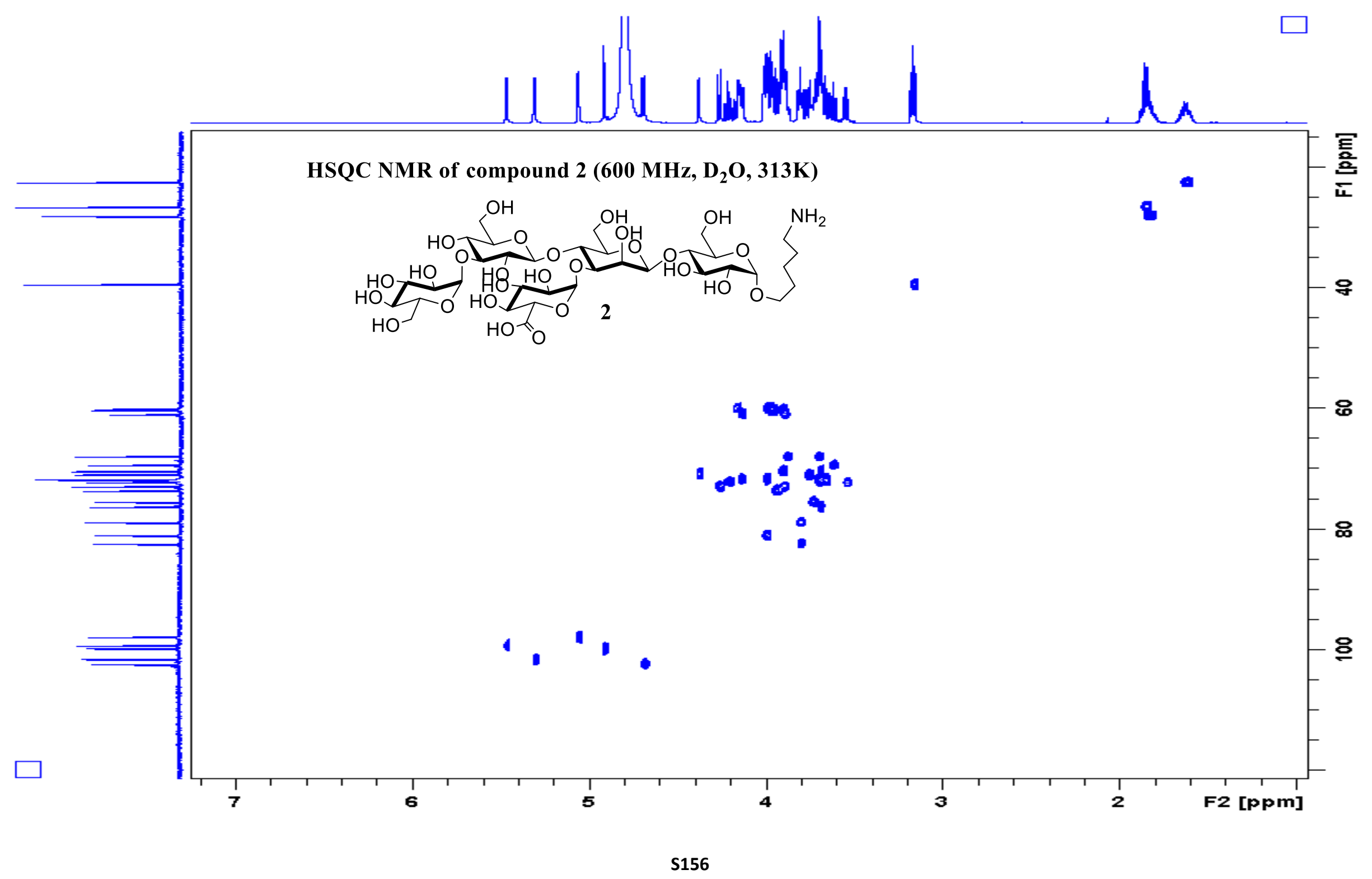




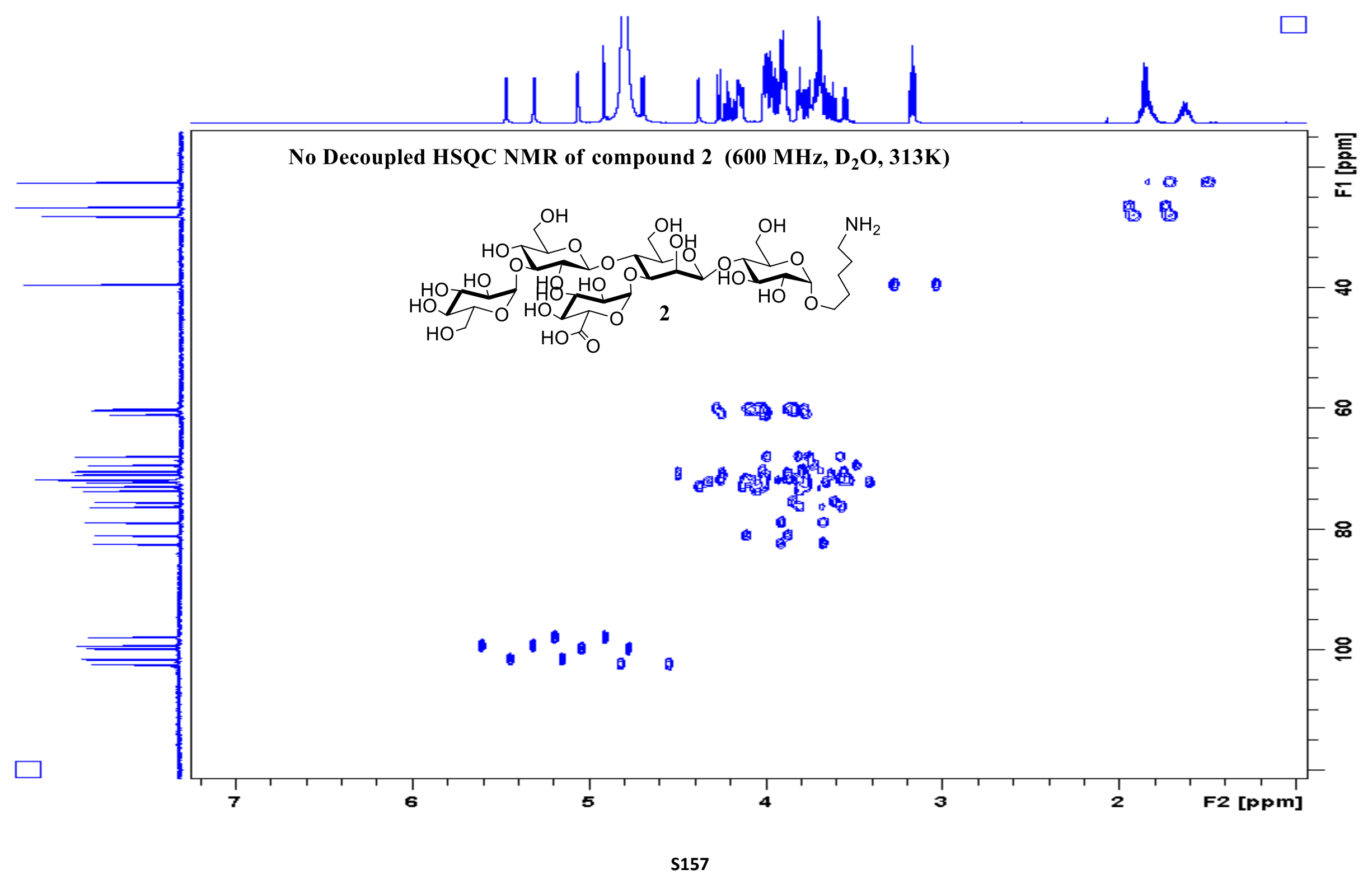




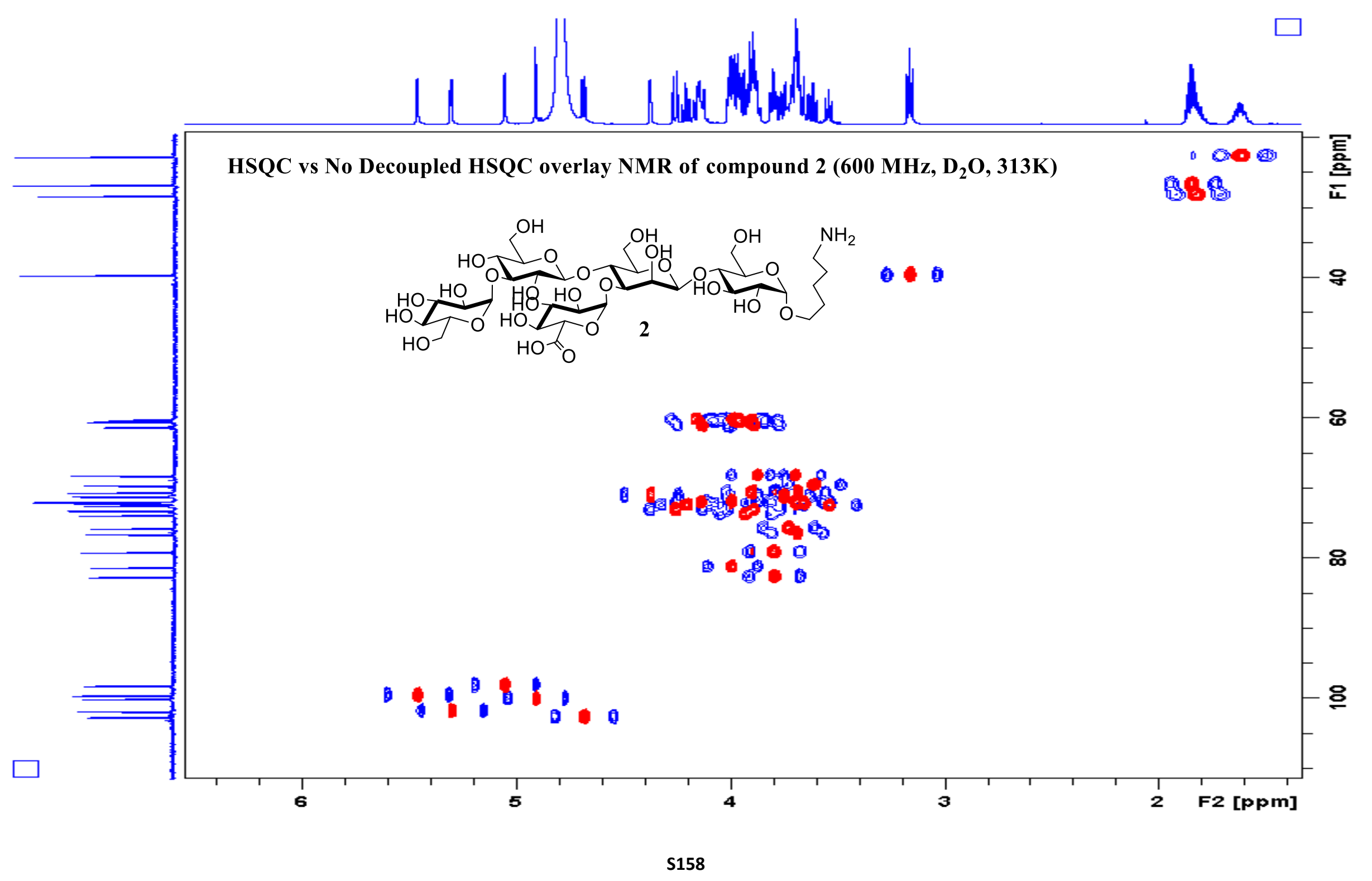




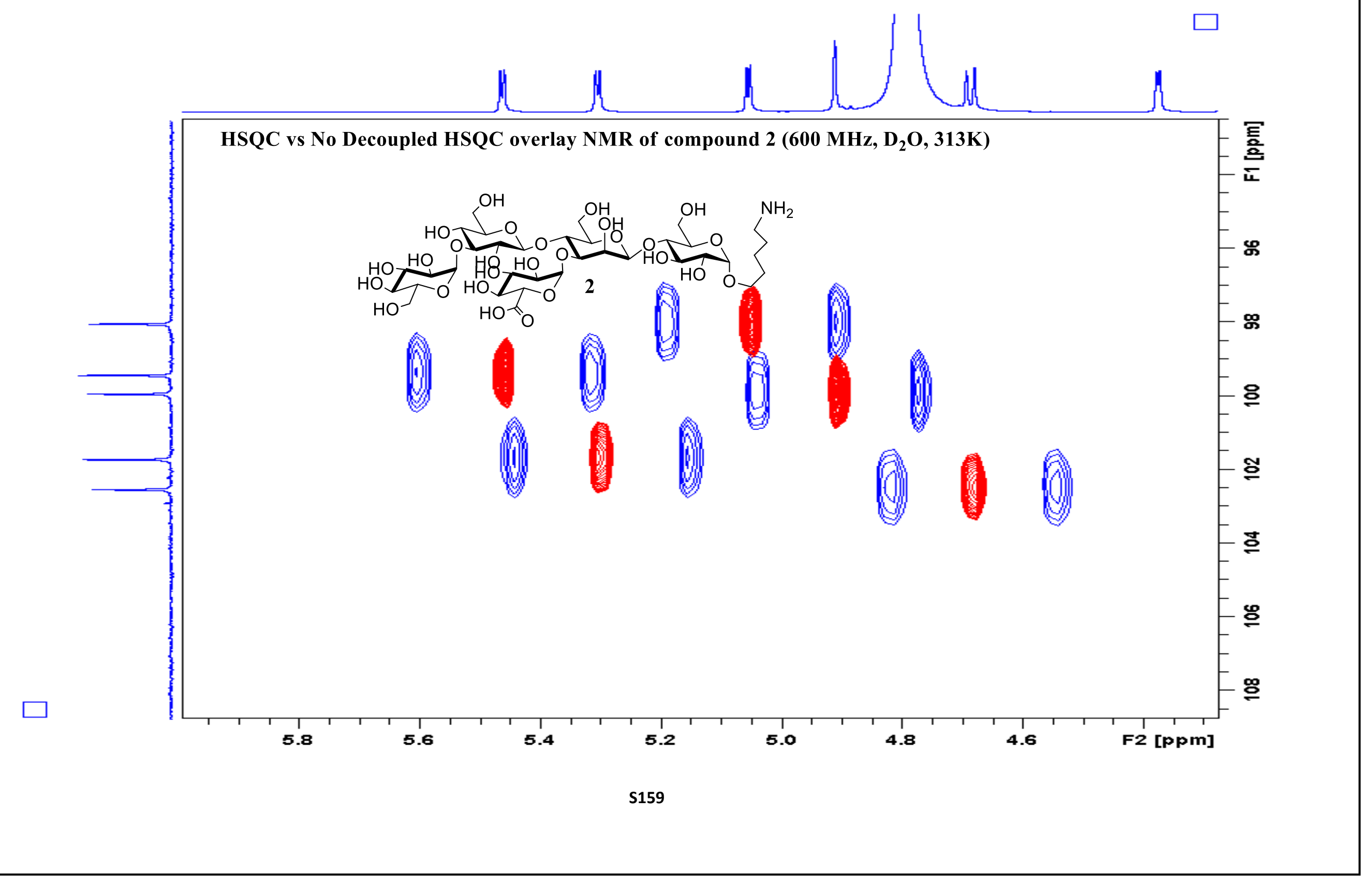




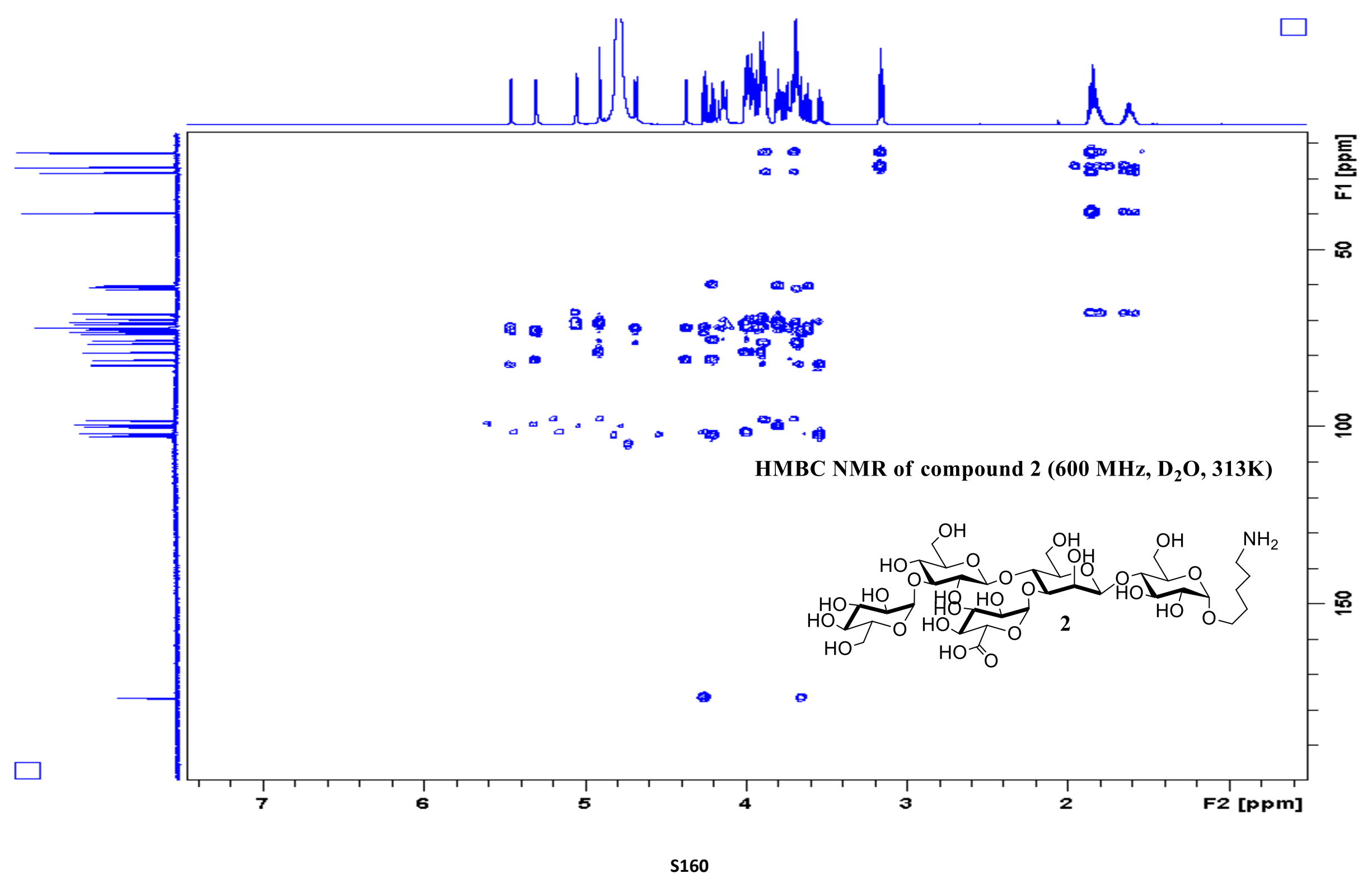




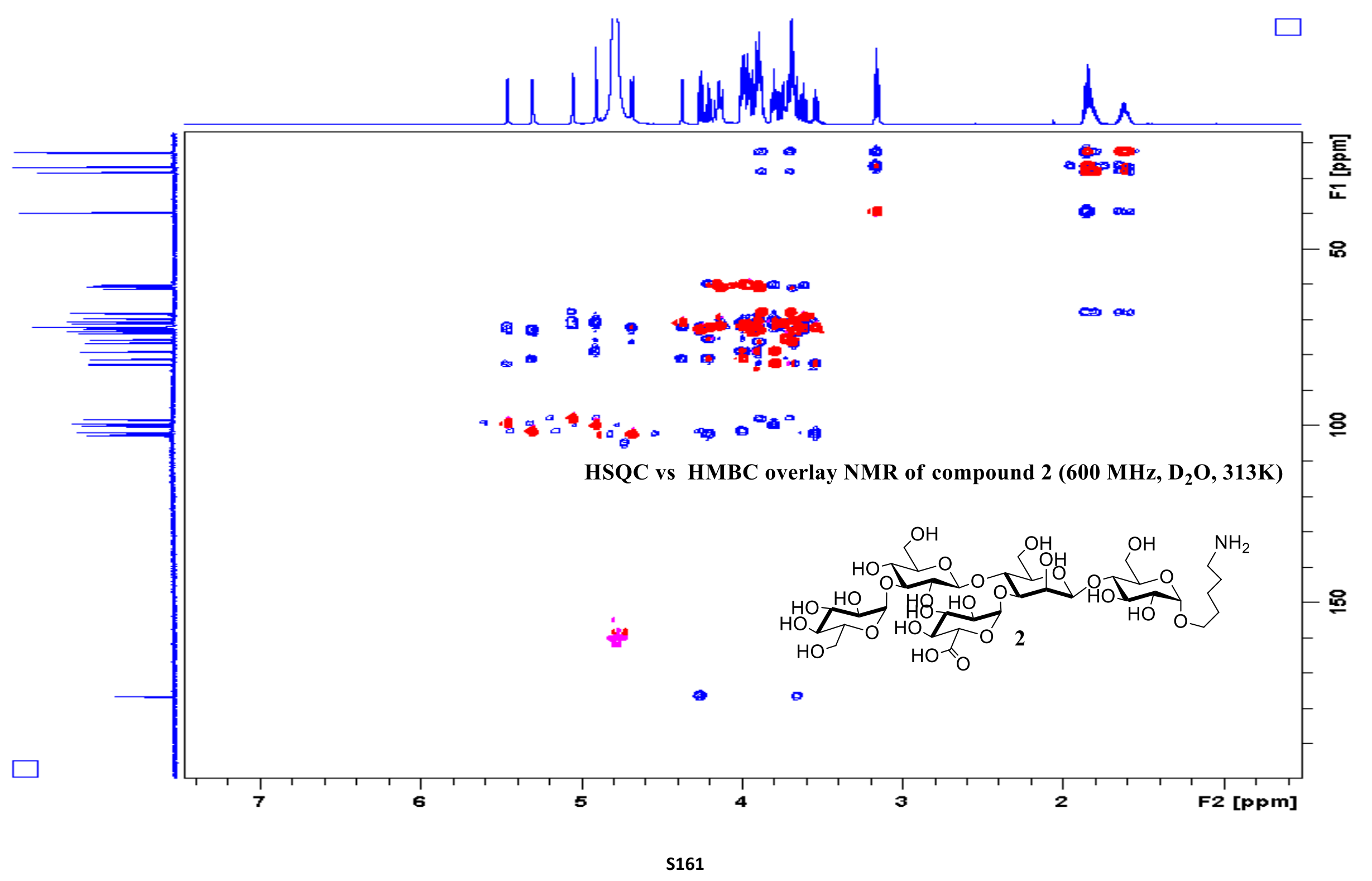




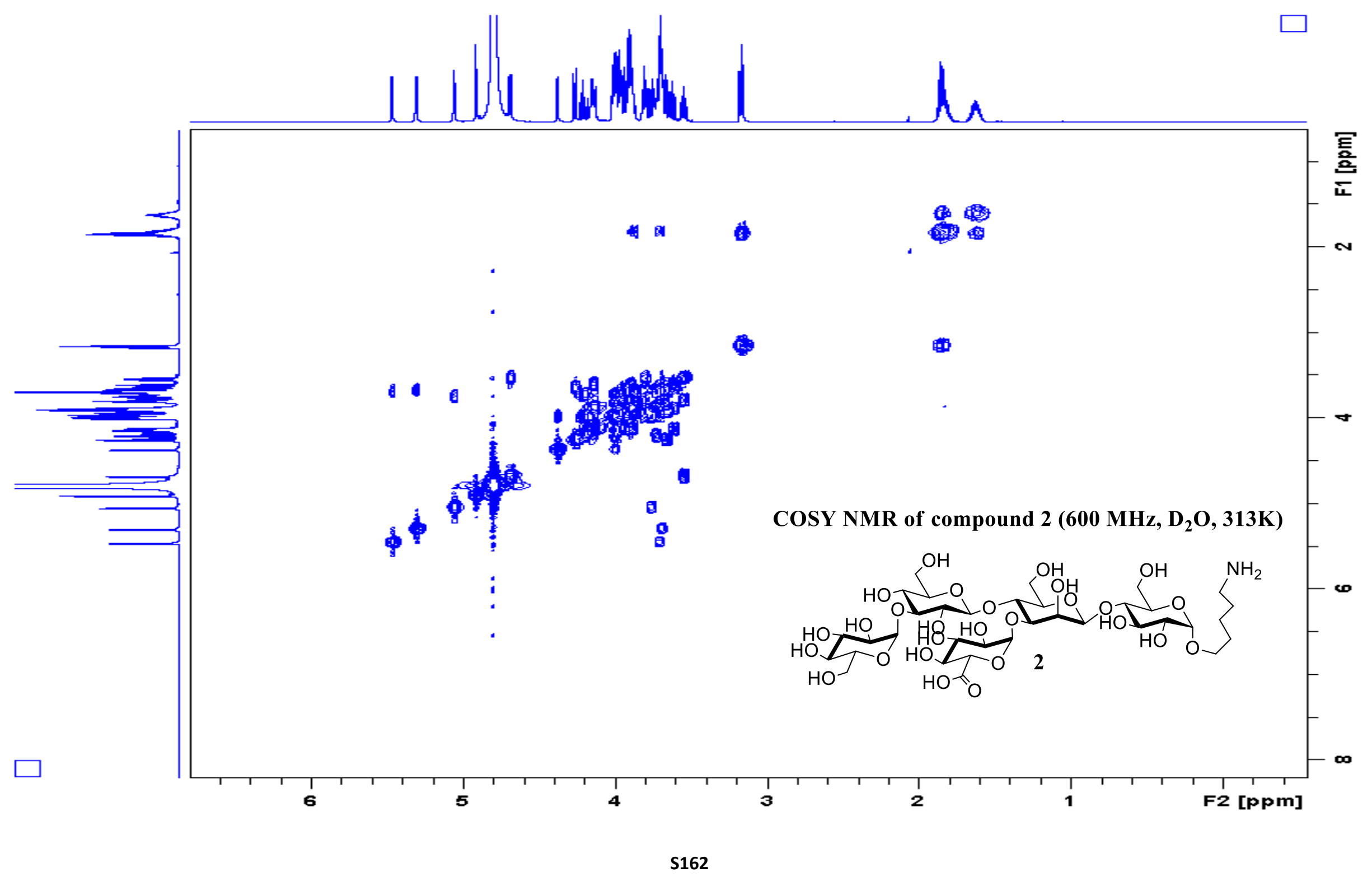


D:I+0IRaw Datal20141107/1109/K2-penta-p

11/7/2014 5:12:51 PM

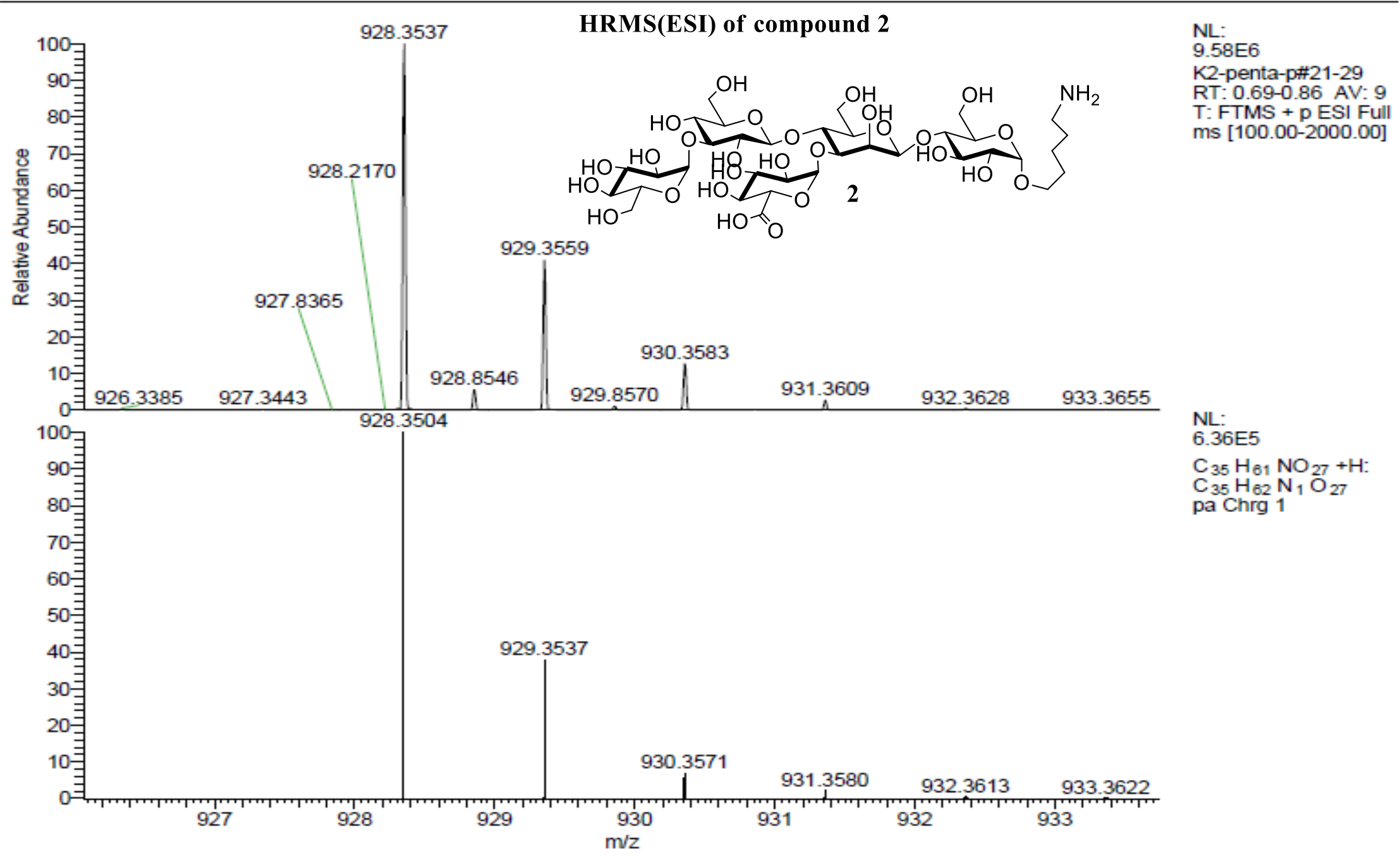




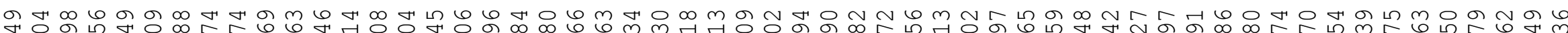

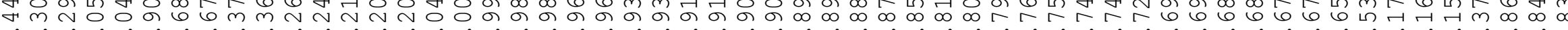

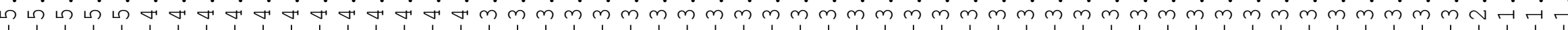

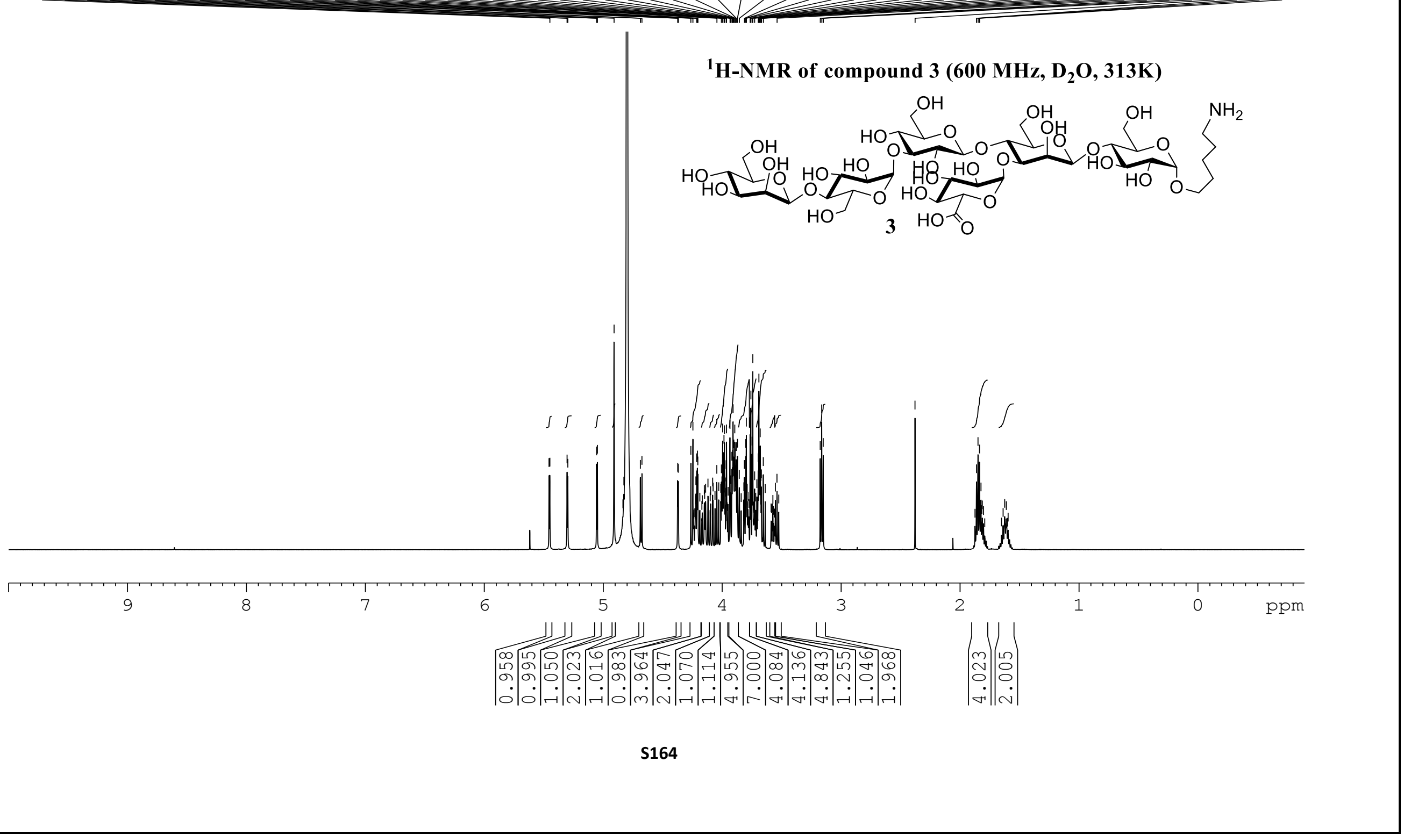


${ }^{13} \mathrm{C}-\mathrm{NMR}$ of compound 3 (150 MHz, $\left.\mathrm{D}_{2} \mathrm{O}, 313 \mathrm{~K}\right)$
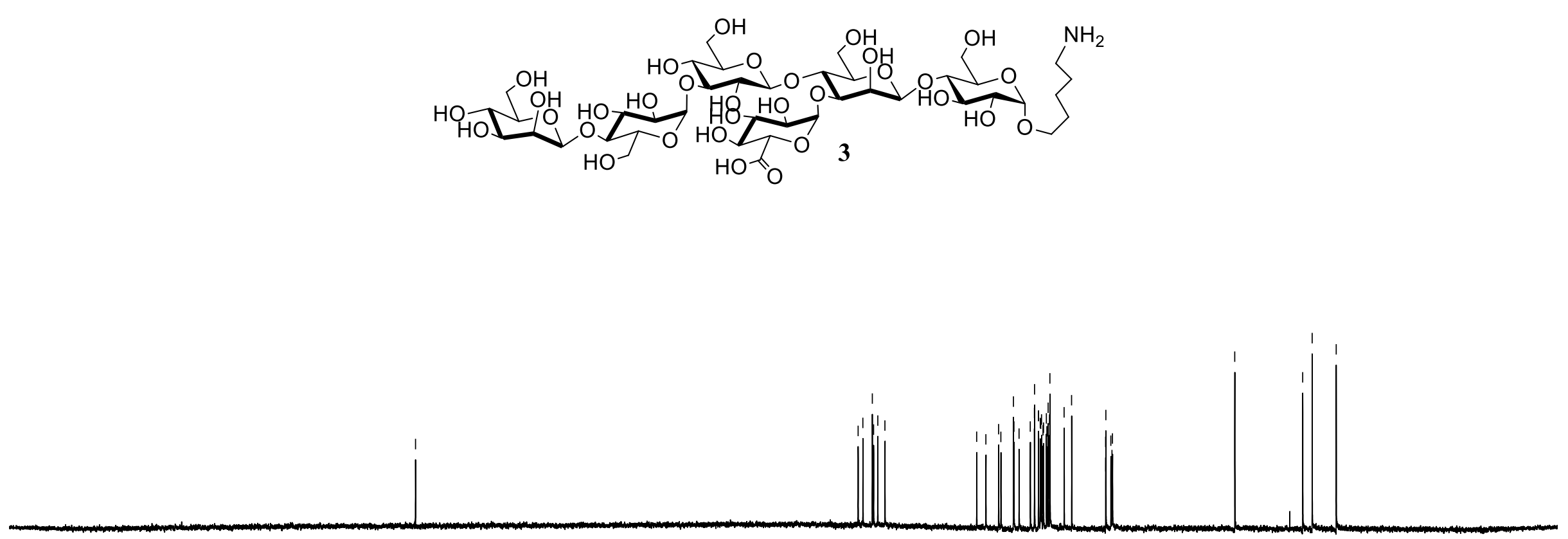


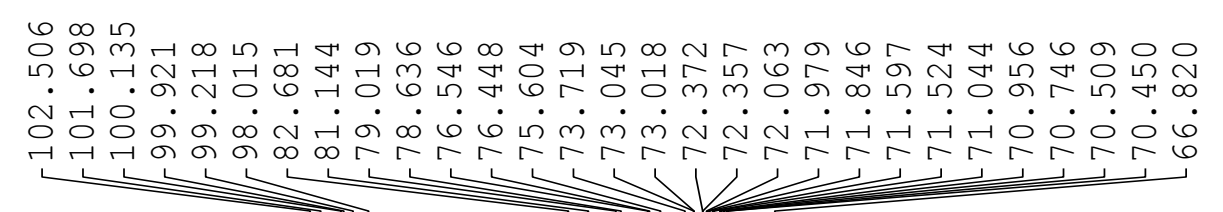

DEPT 135 NMR of compound $3\left(150 \mathrm{MHz}, \mathrm{D}_{2} \mathrm{O}, 313 \mathrm{~K}\right)$

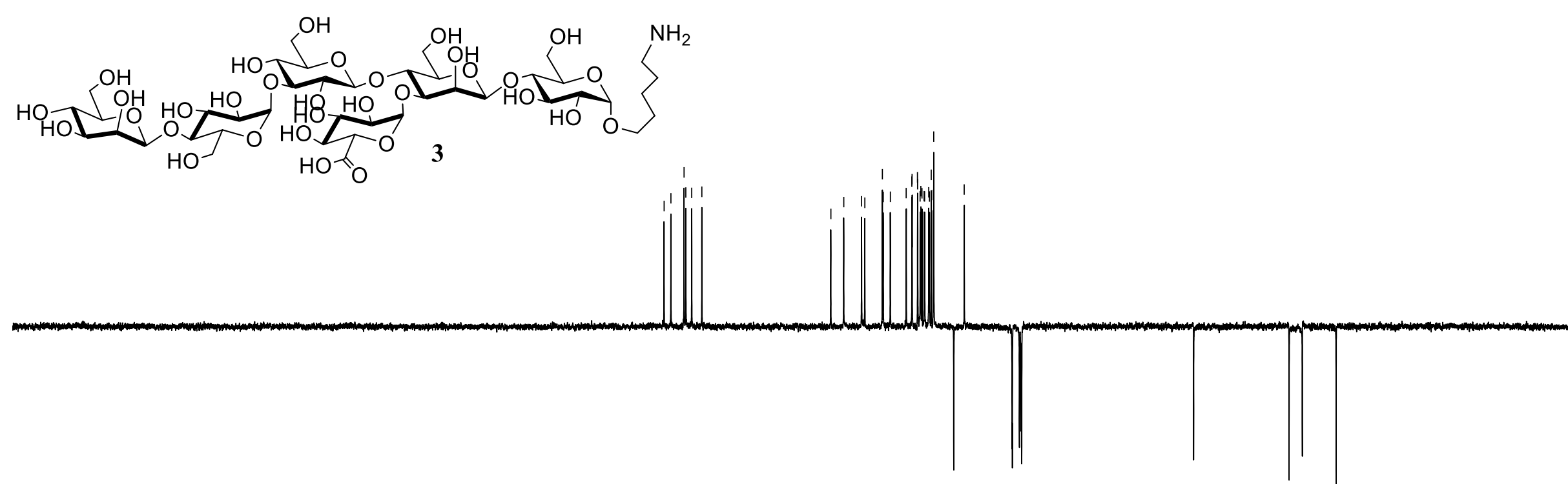

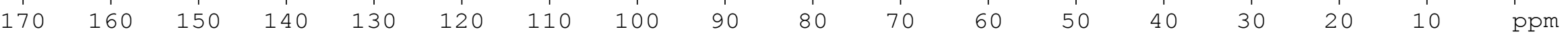




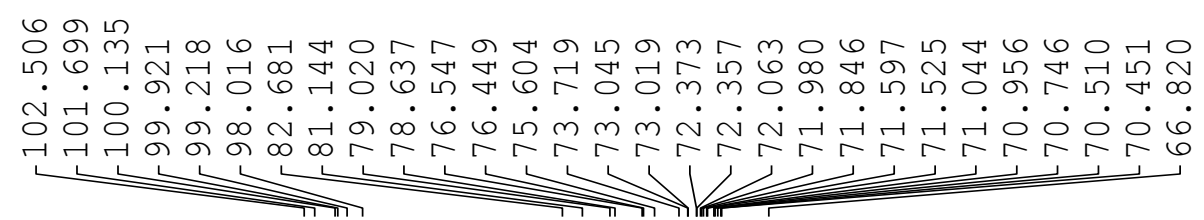

DEPT 90 NMR of compound $3\left(150 \mathrm{MHz}, \mathrm{D}_{2} \mathrm{O}, 313 \mathrm{~K}\right)$
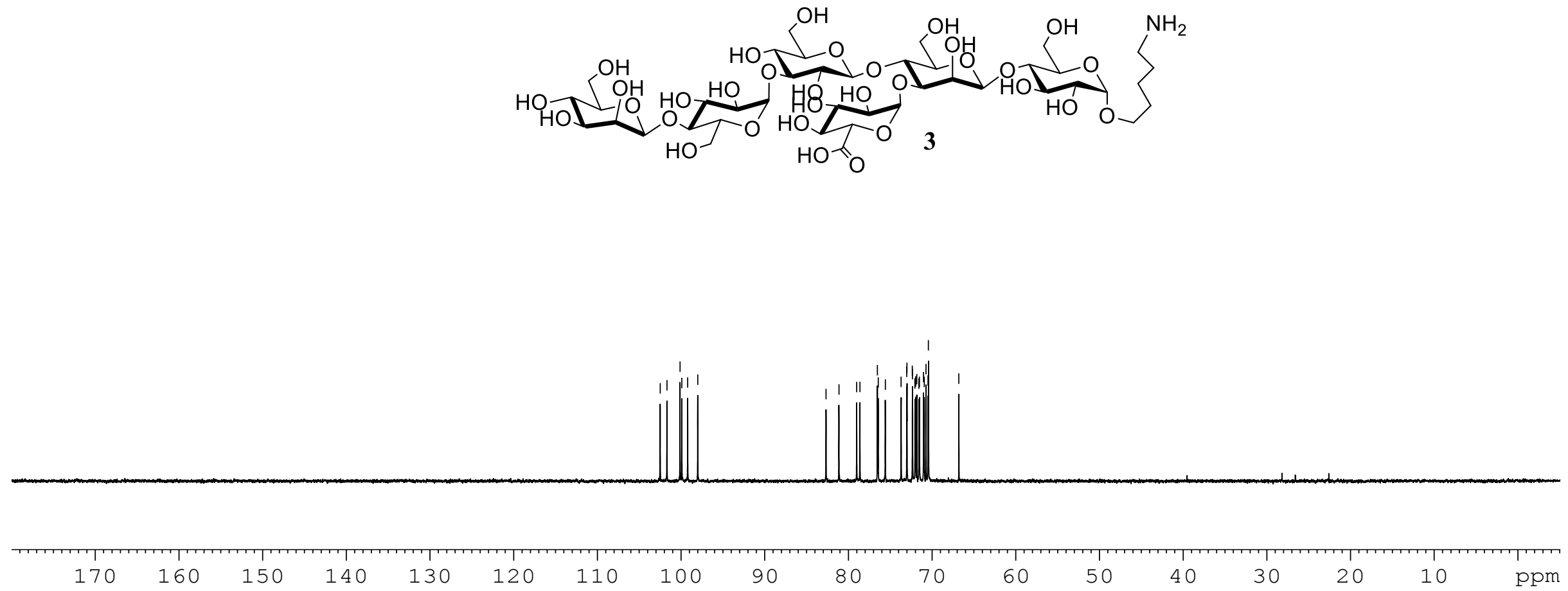


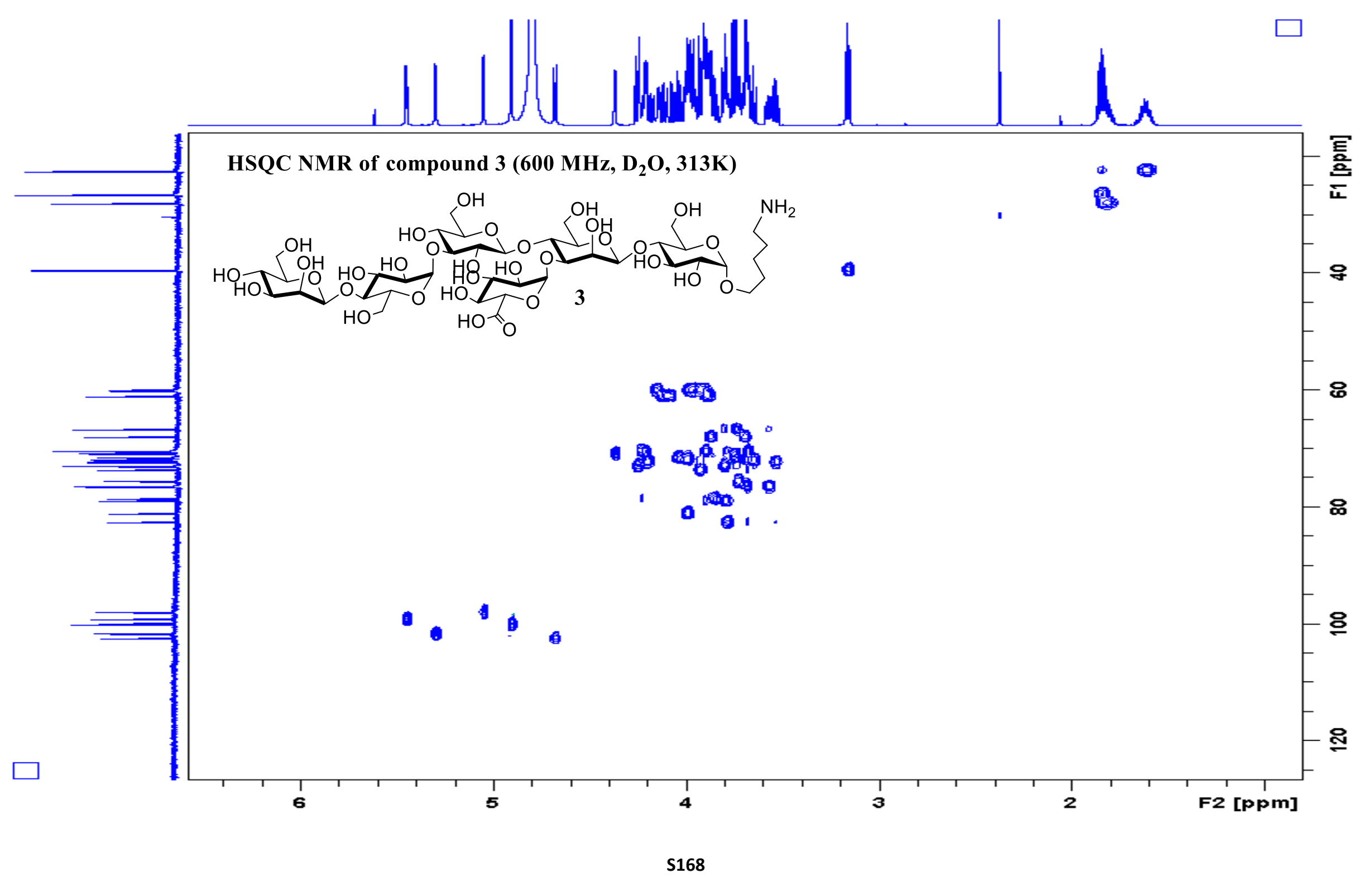




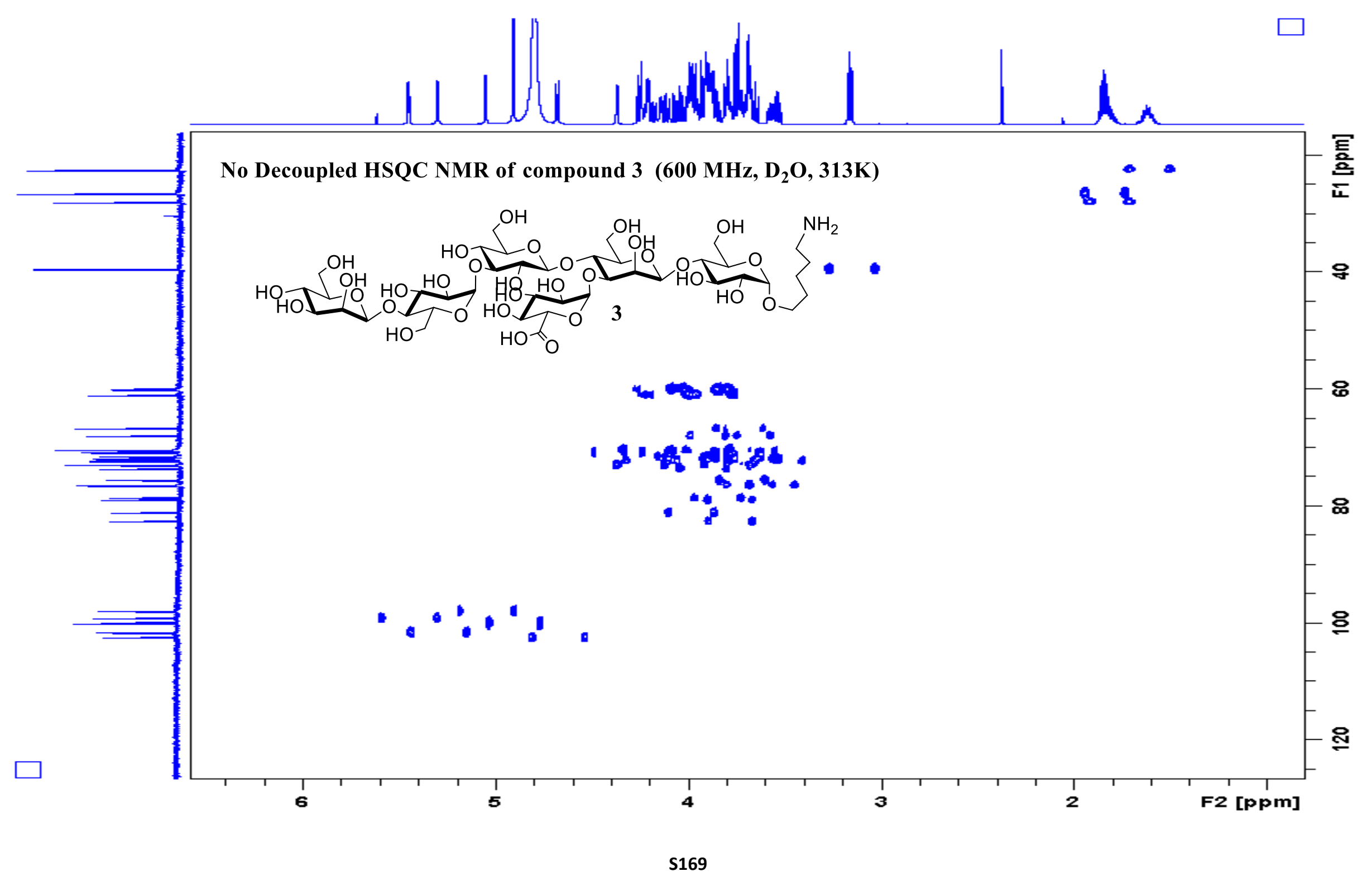




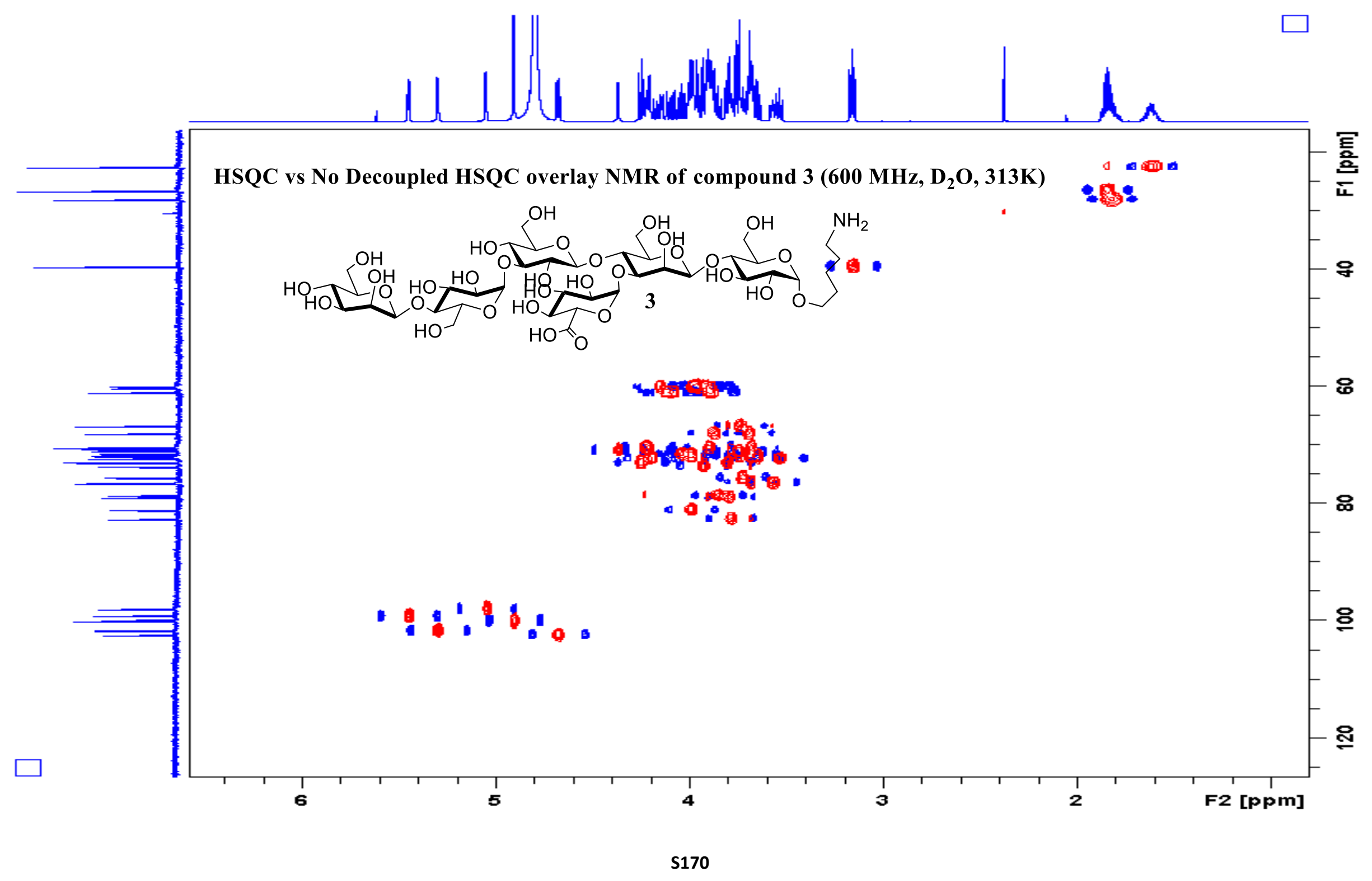




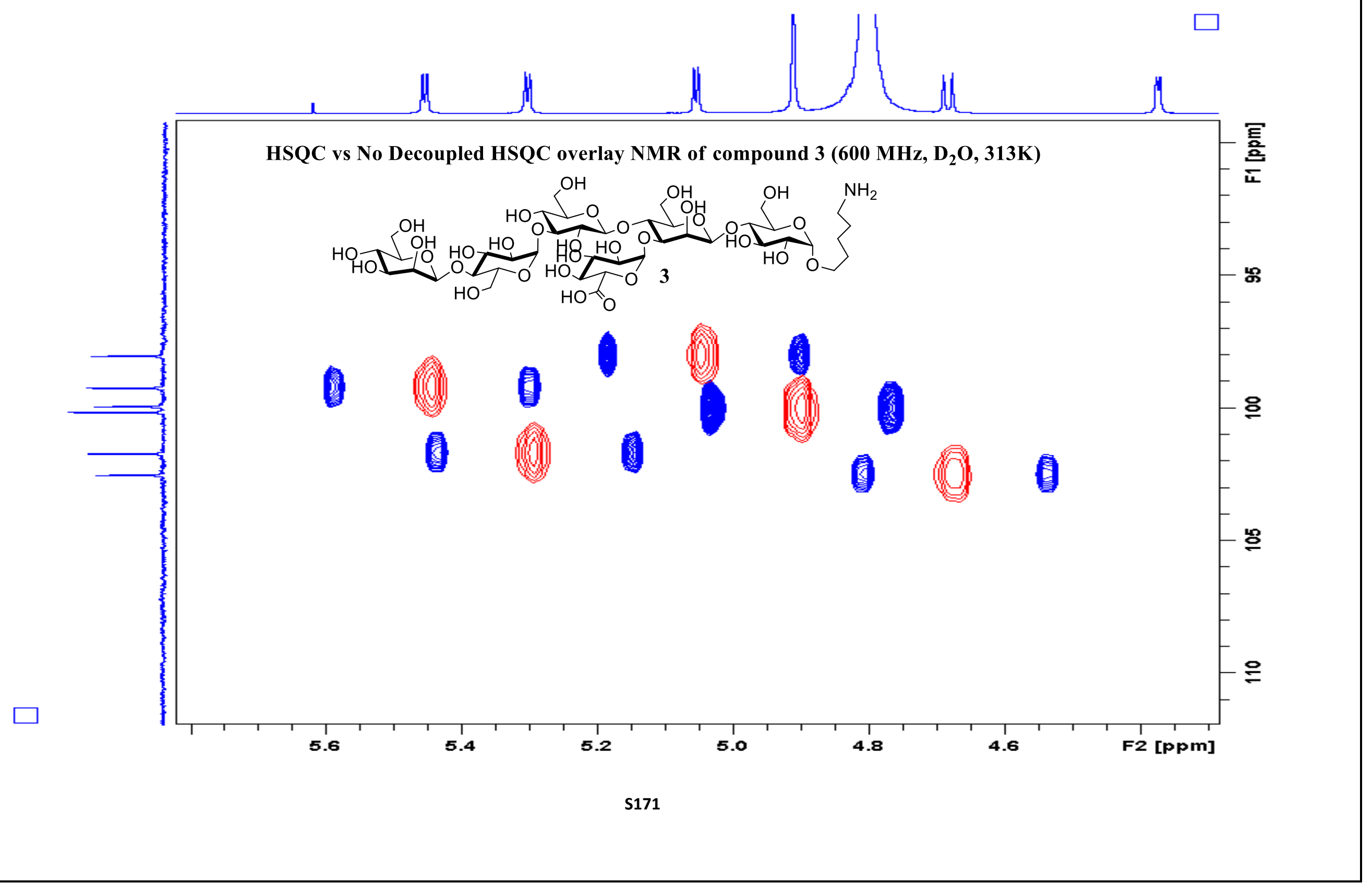




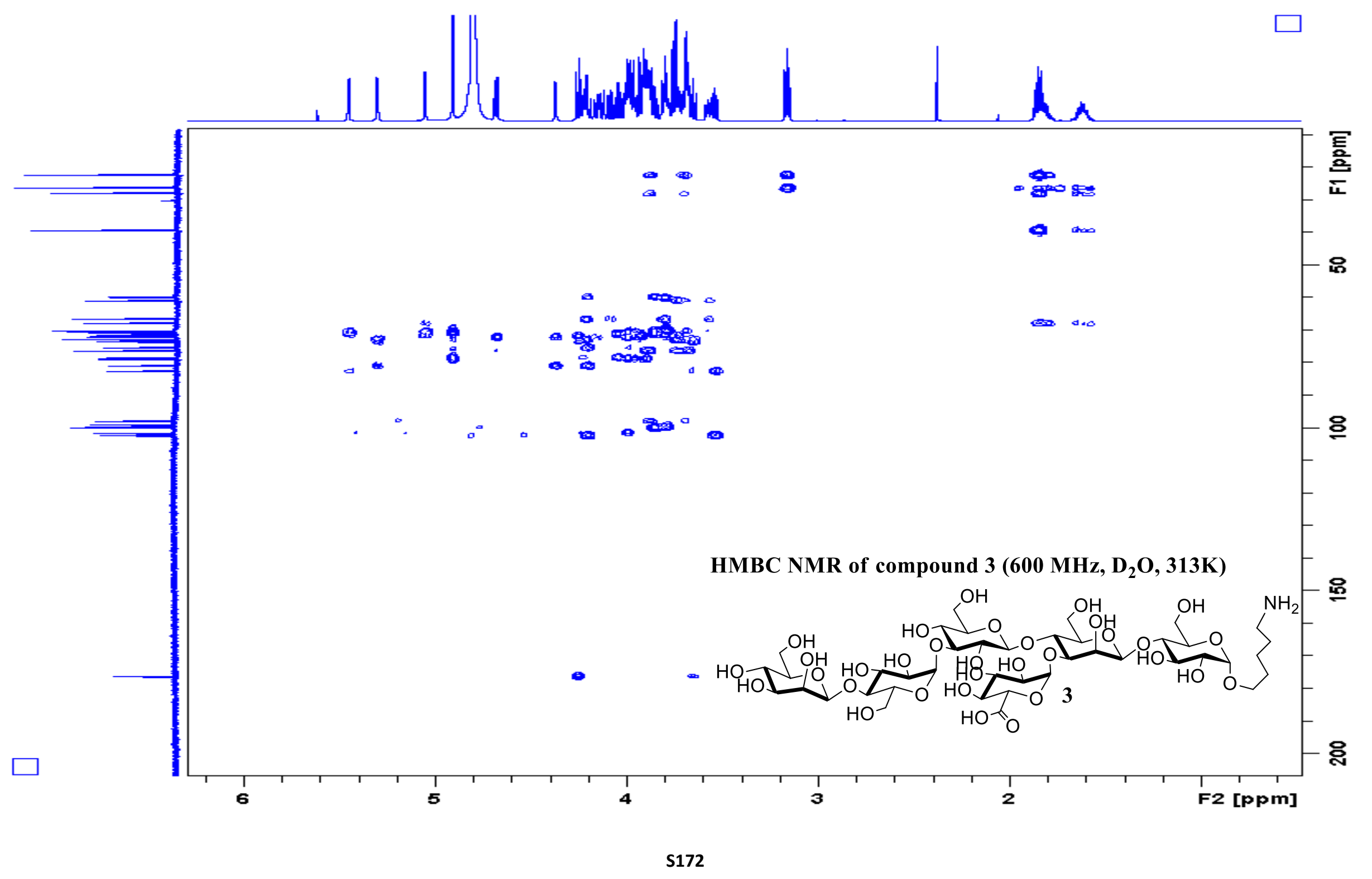




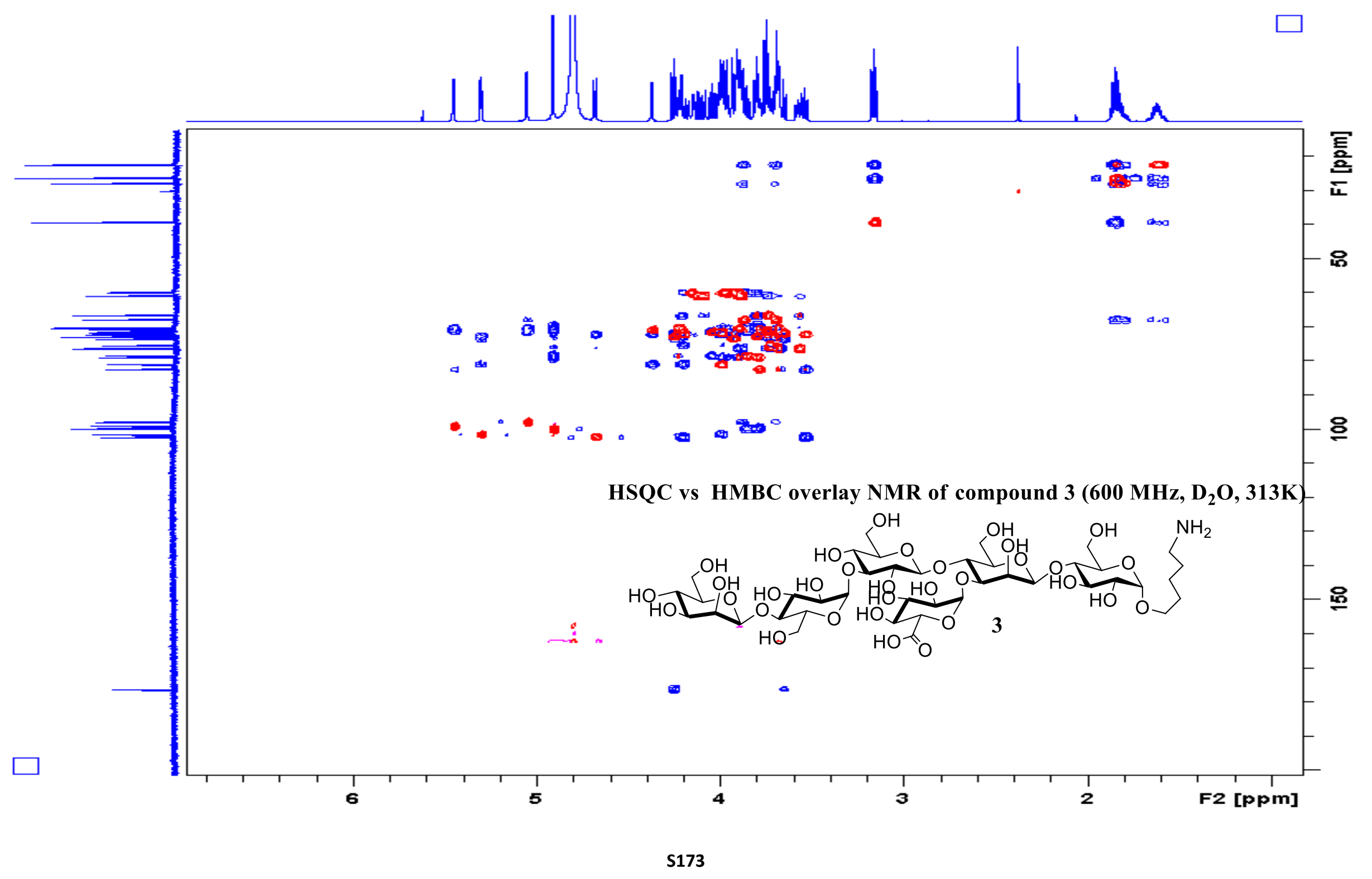




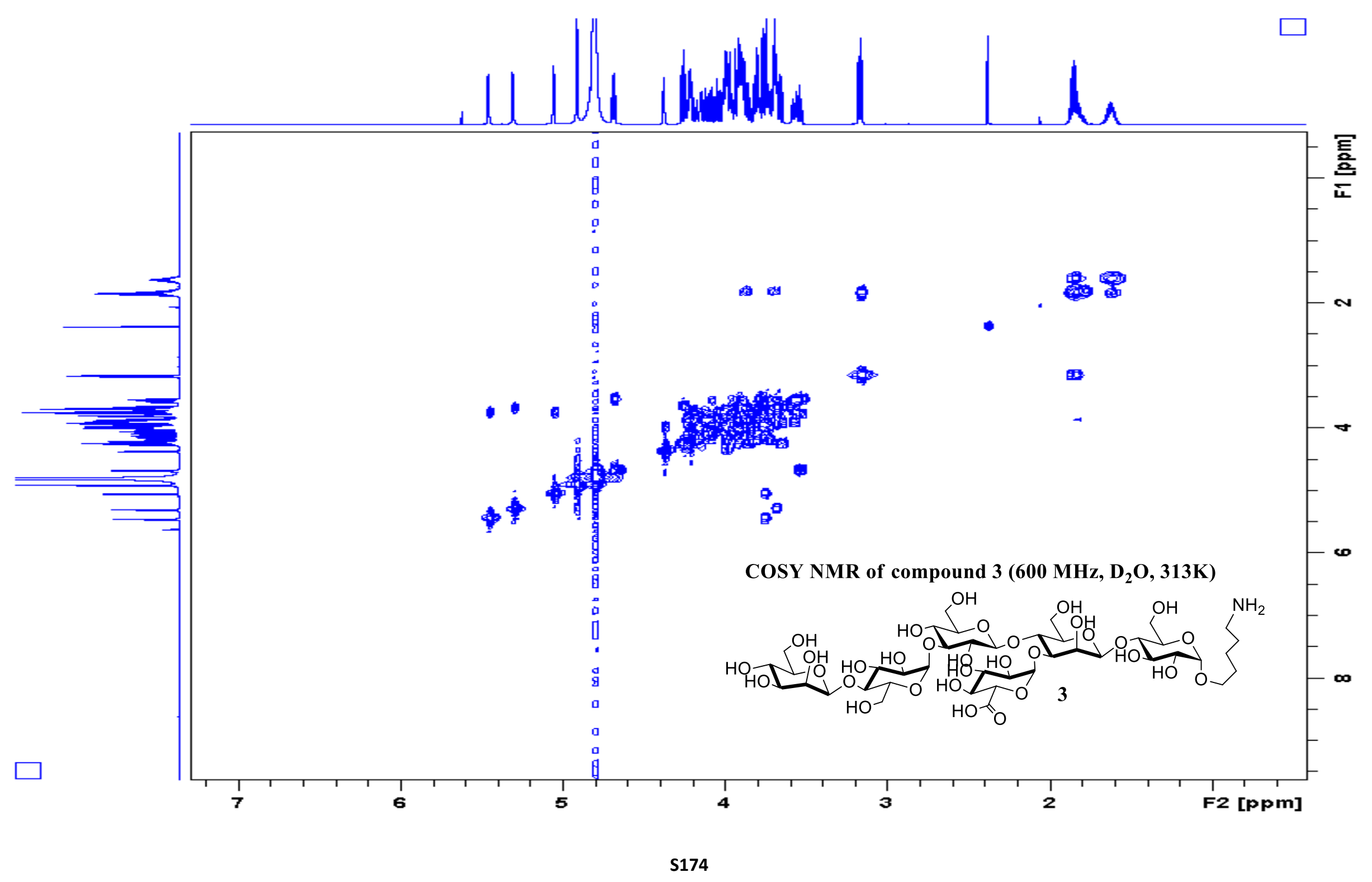




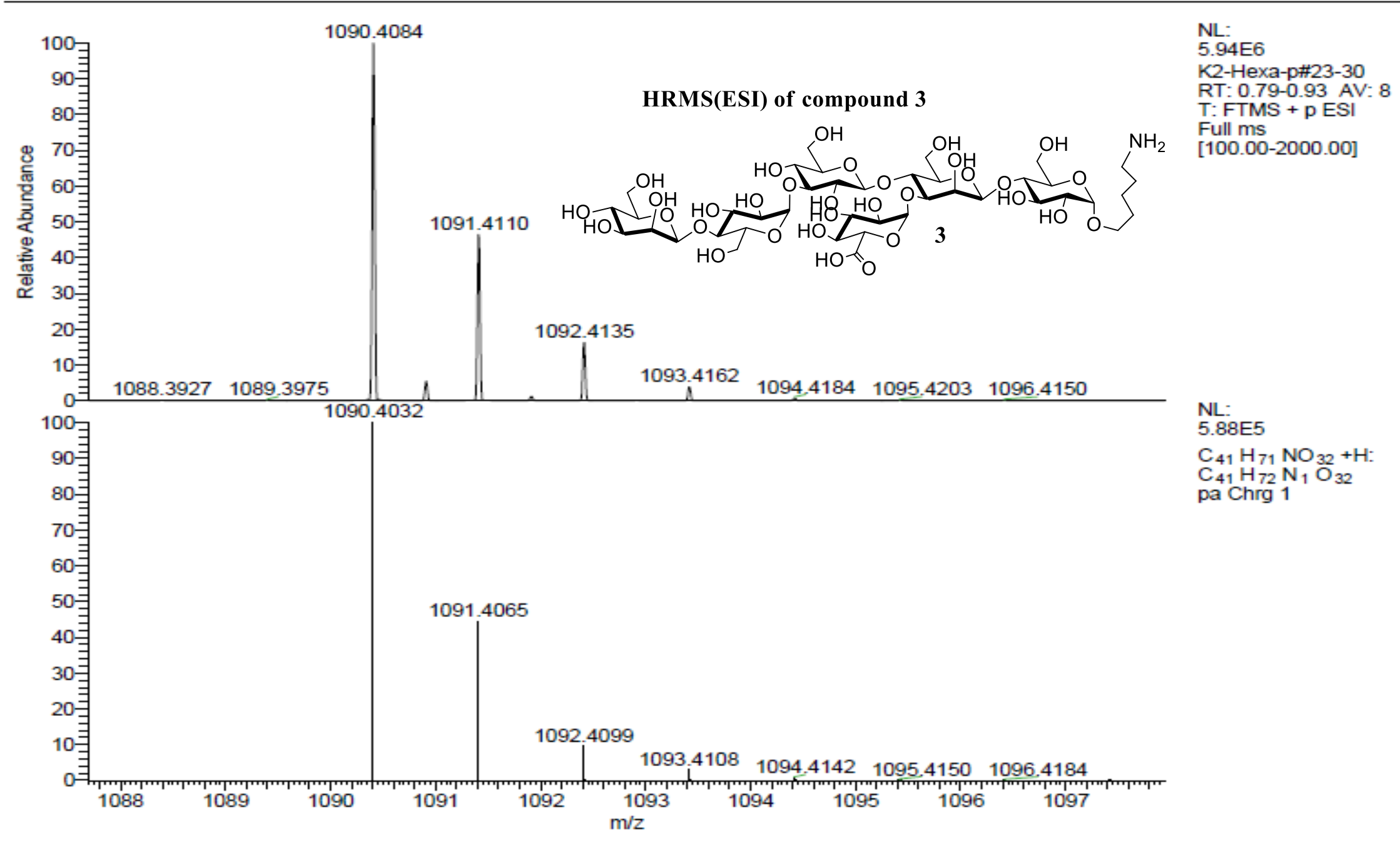




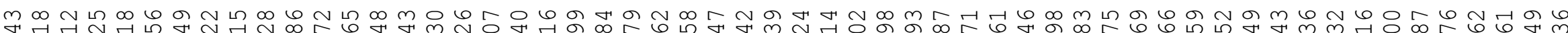

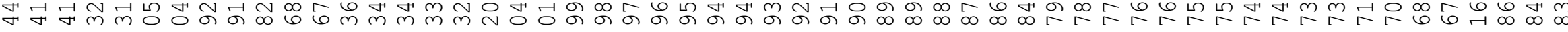

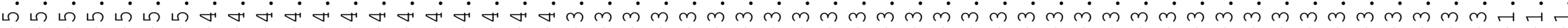

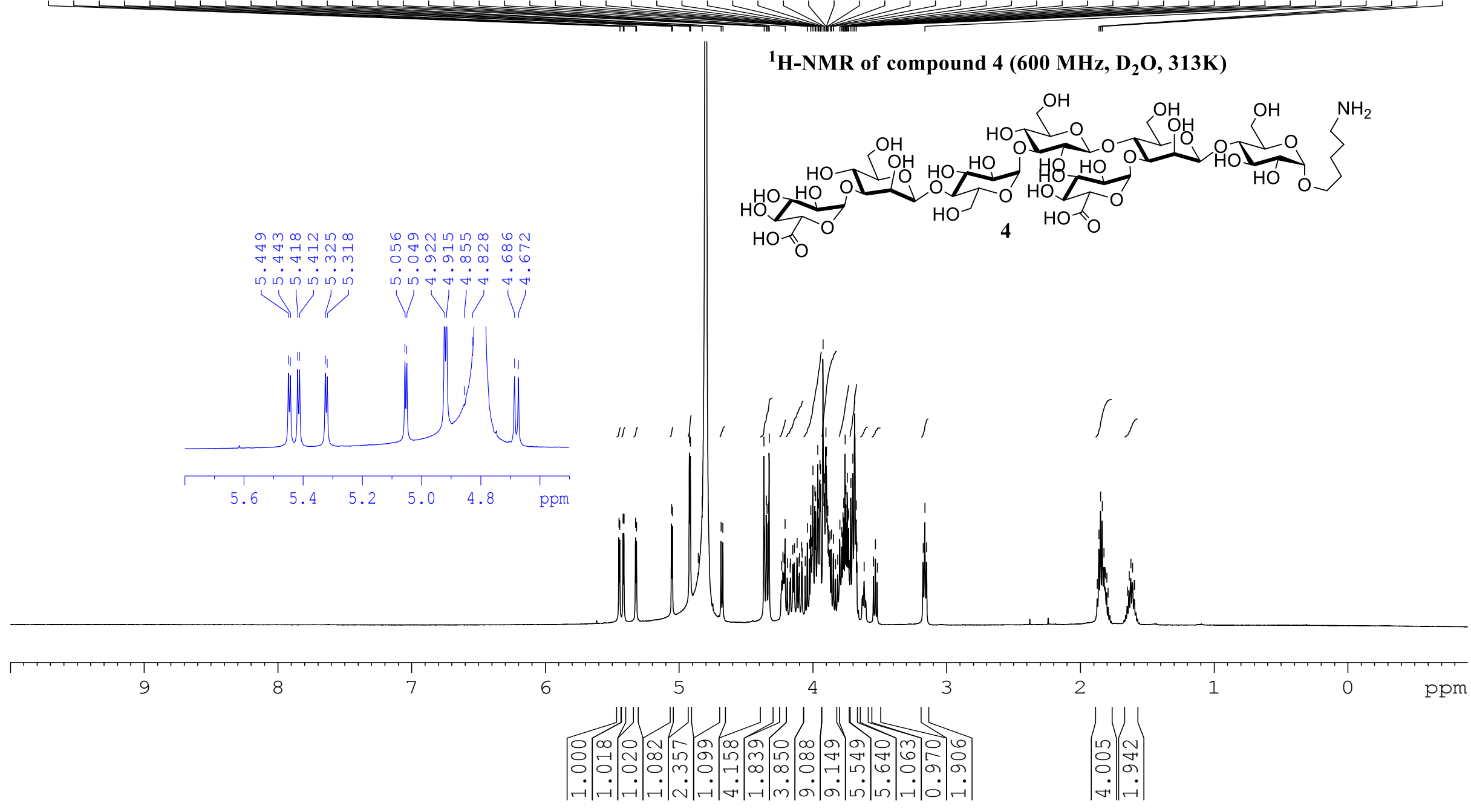


${ }^{13} \mathrm{C}-\mathrm{NMR}$ of compound 4 (150 MHz, $\left.\mathrm{D}_{2} \mathrm{O}, 313 \mathrm{~K}\right)$

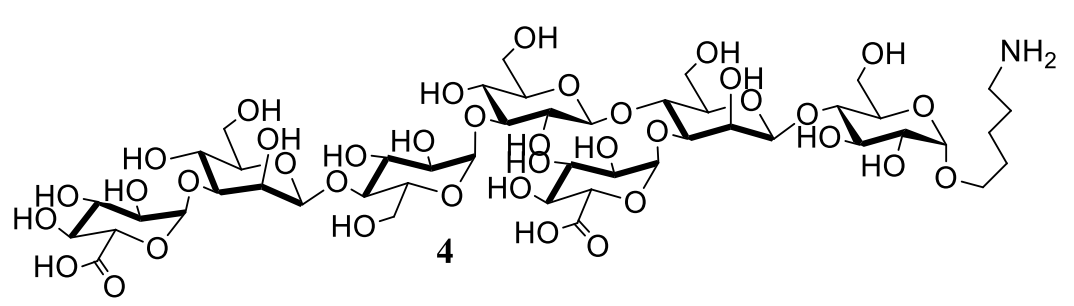

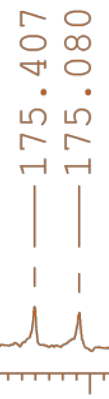

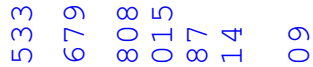

. $\cdot . r \sim$

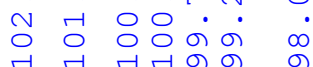

i
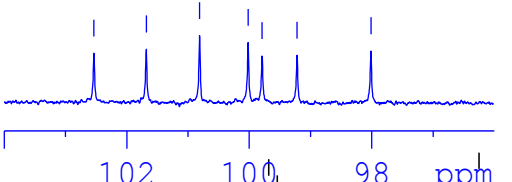

piom

102
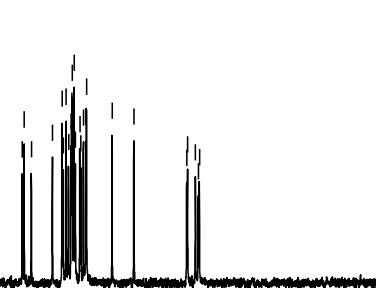


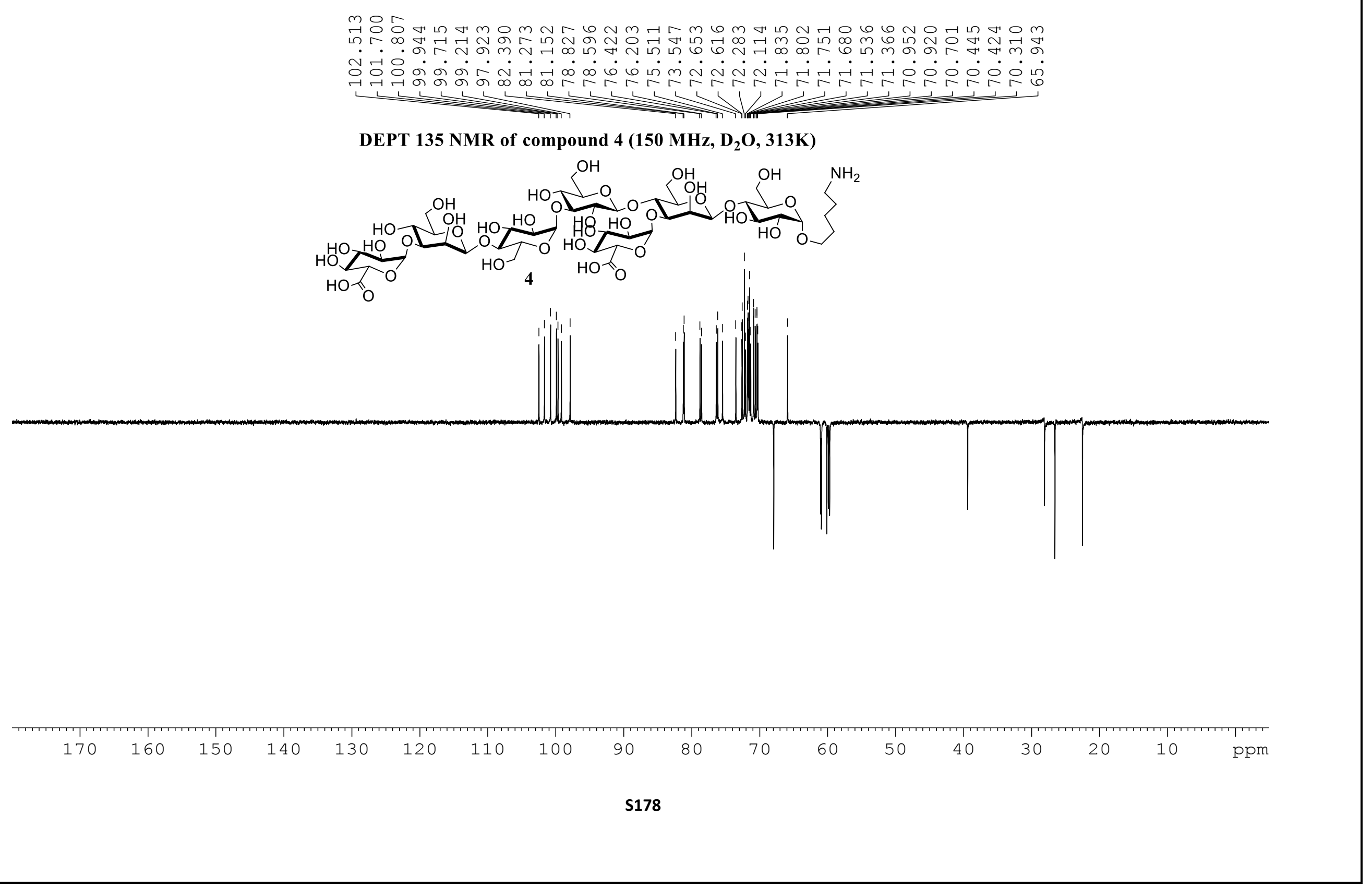




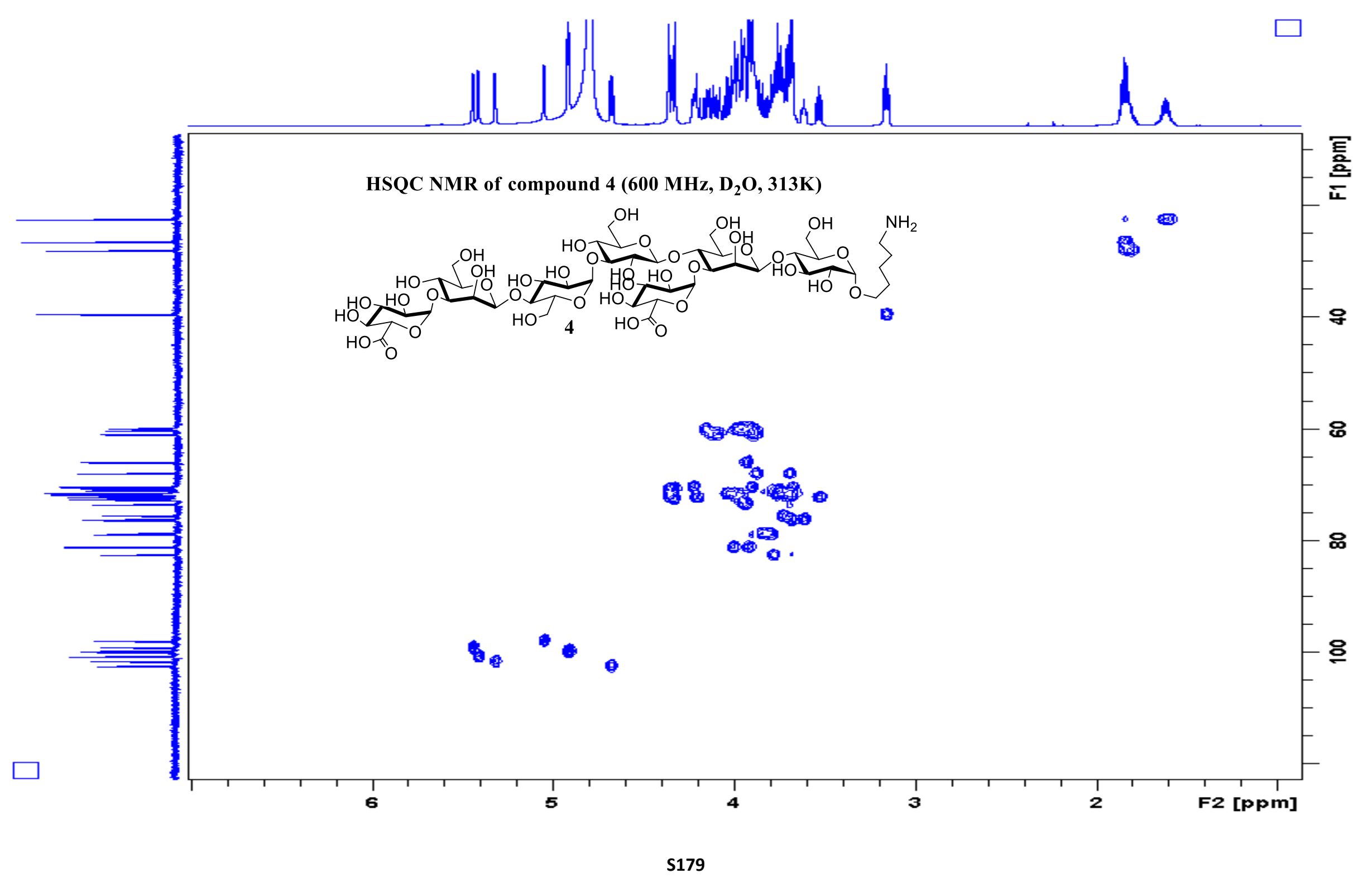




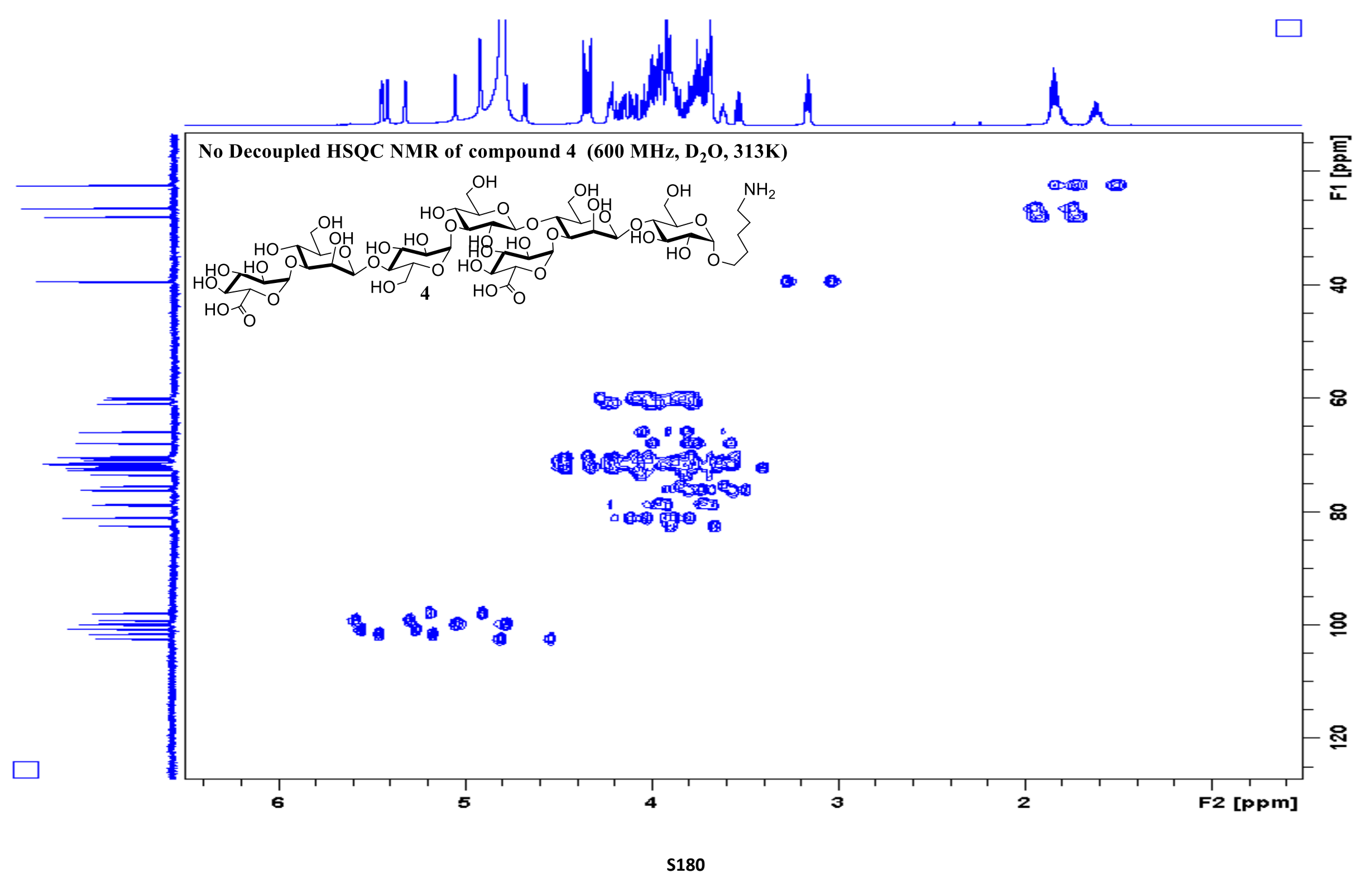




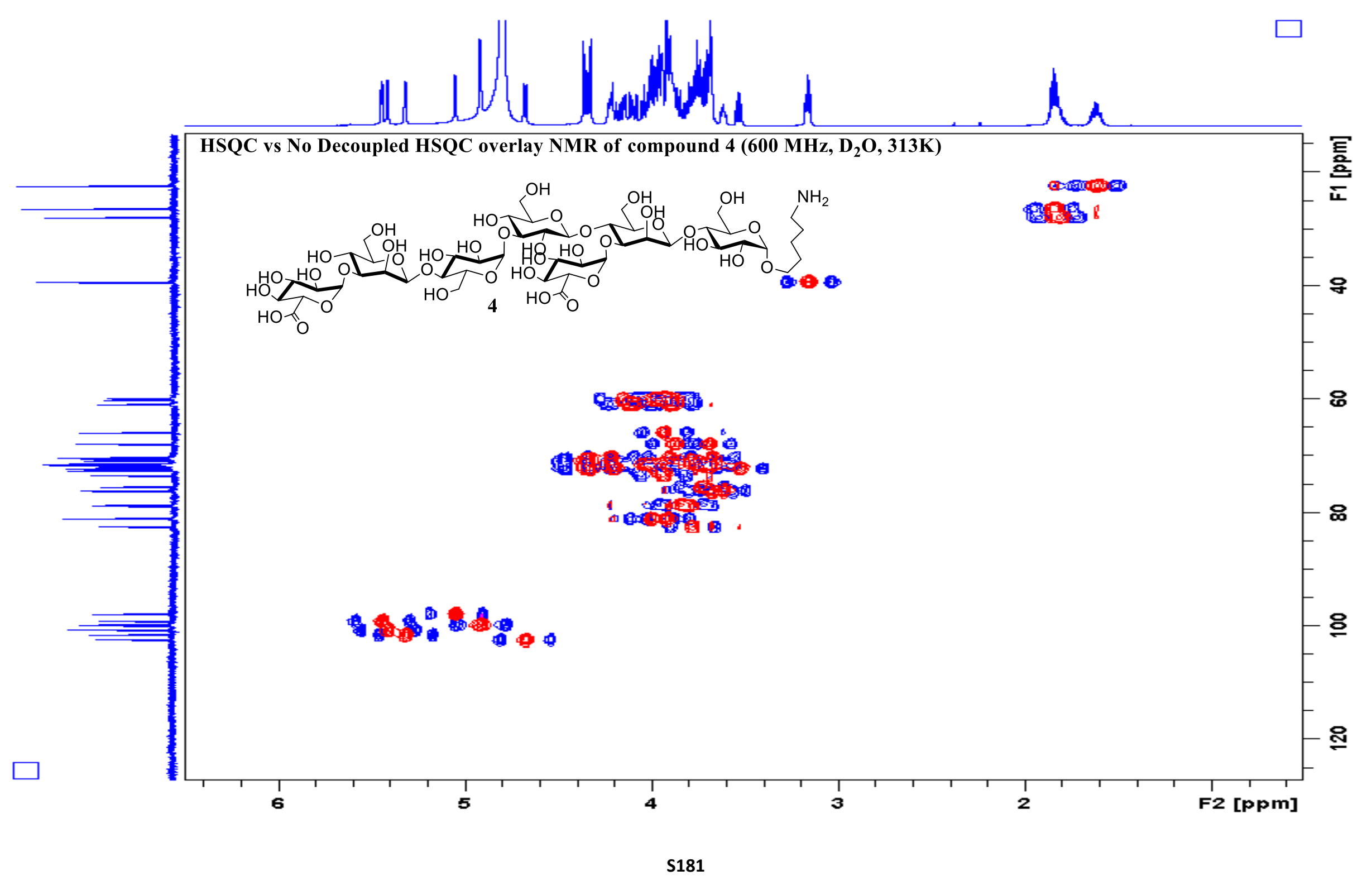




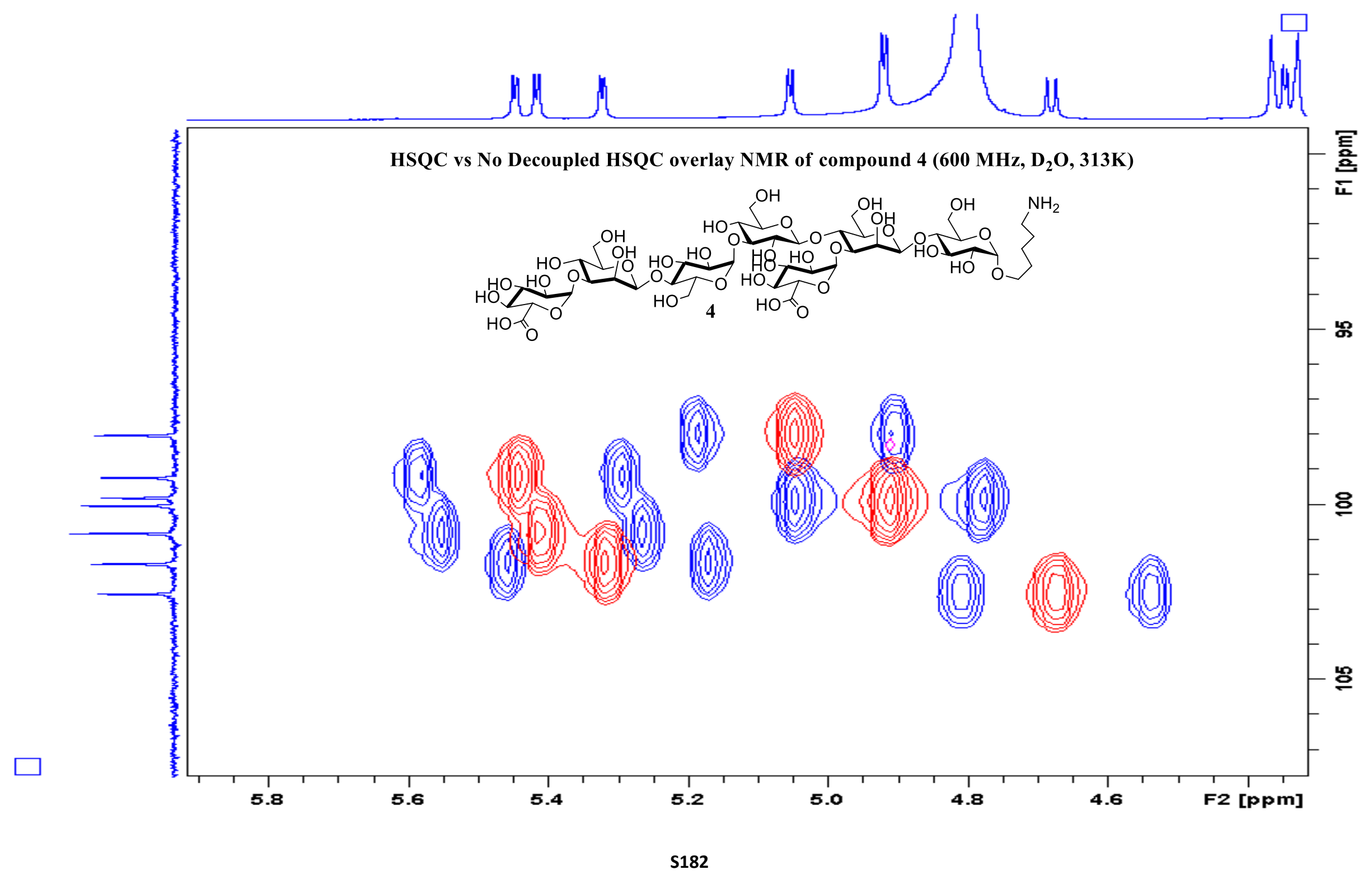




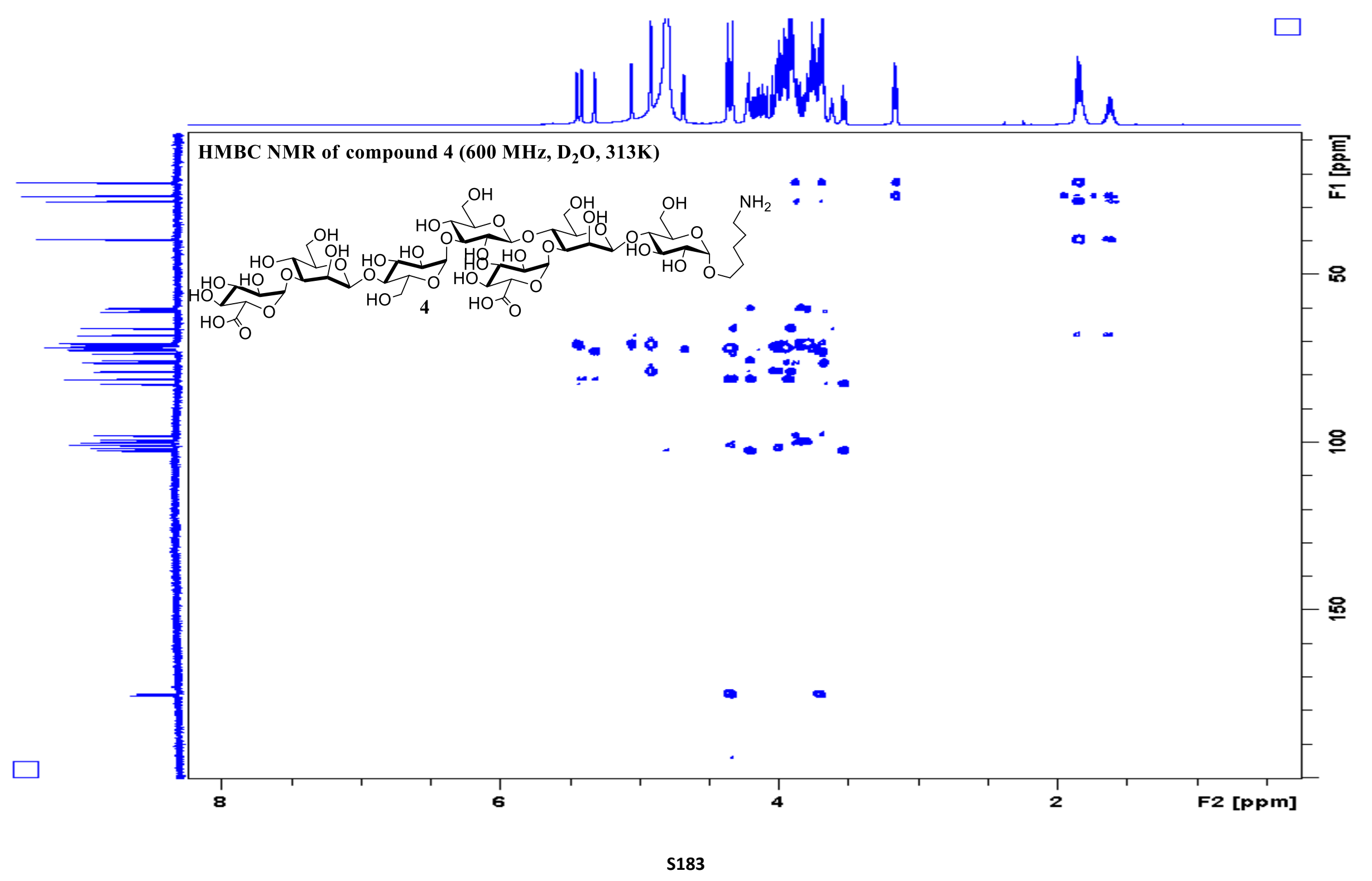




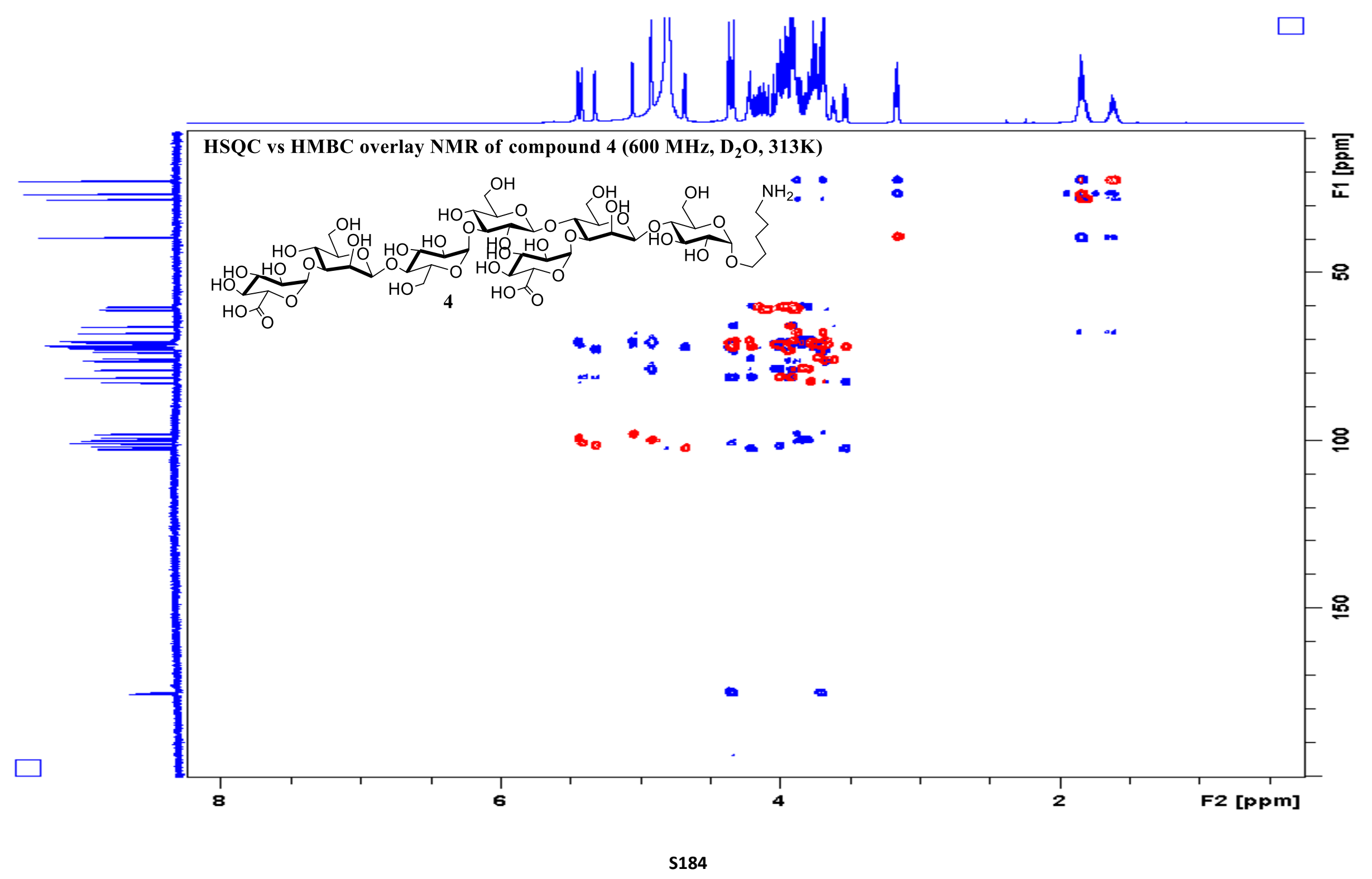




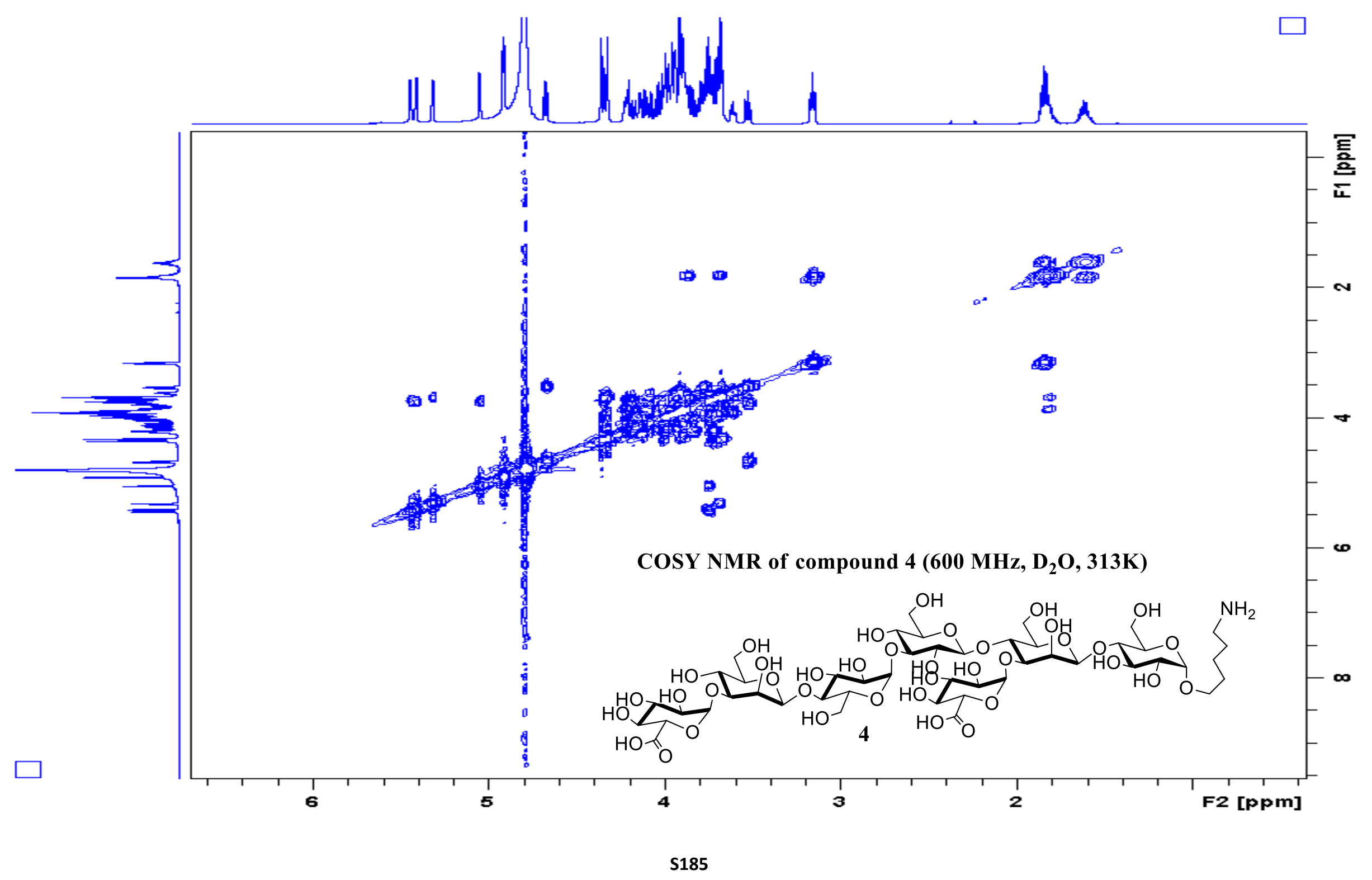




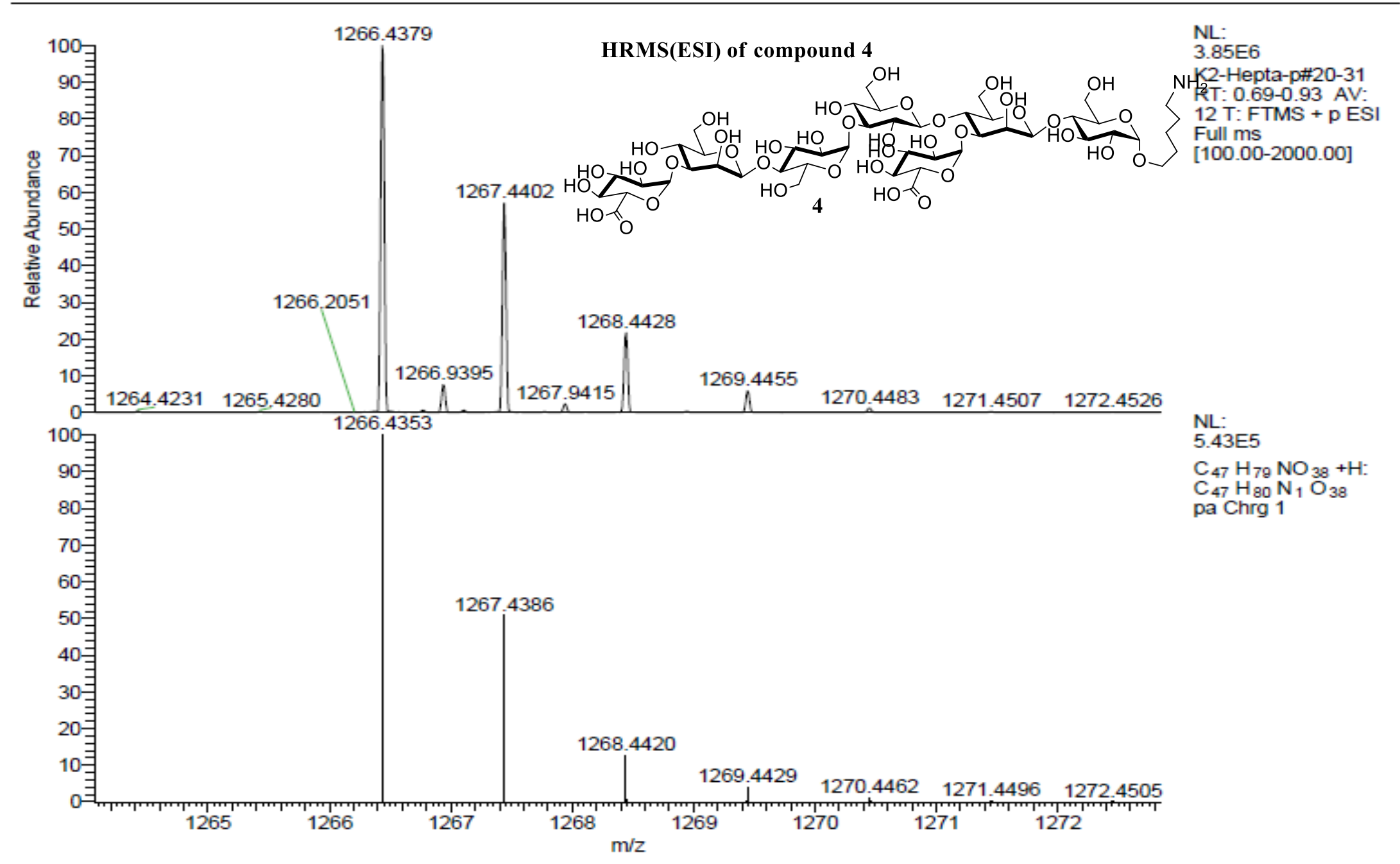




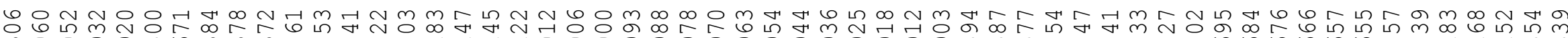
m. 잉. o. o.

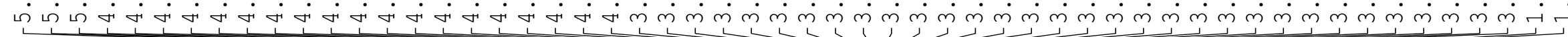

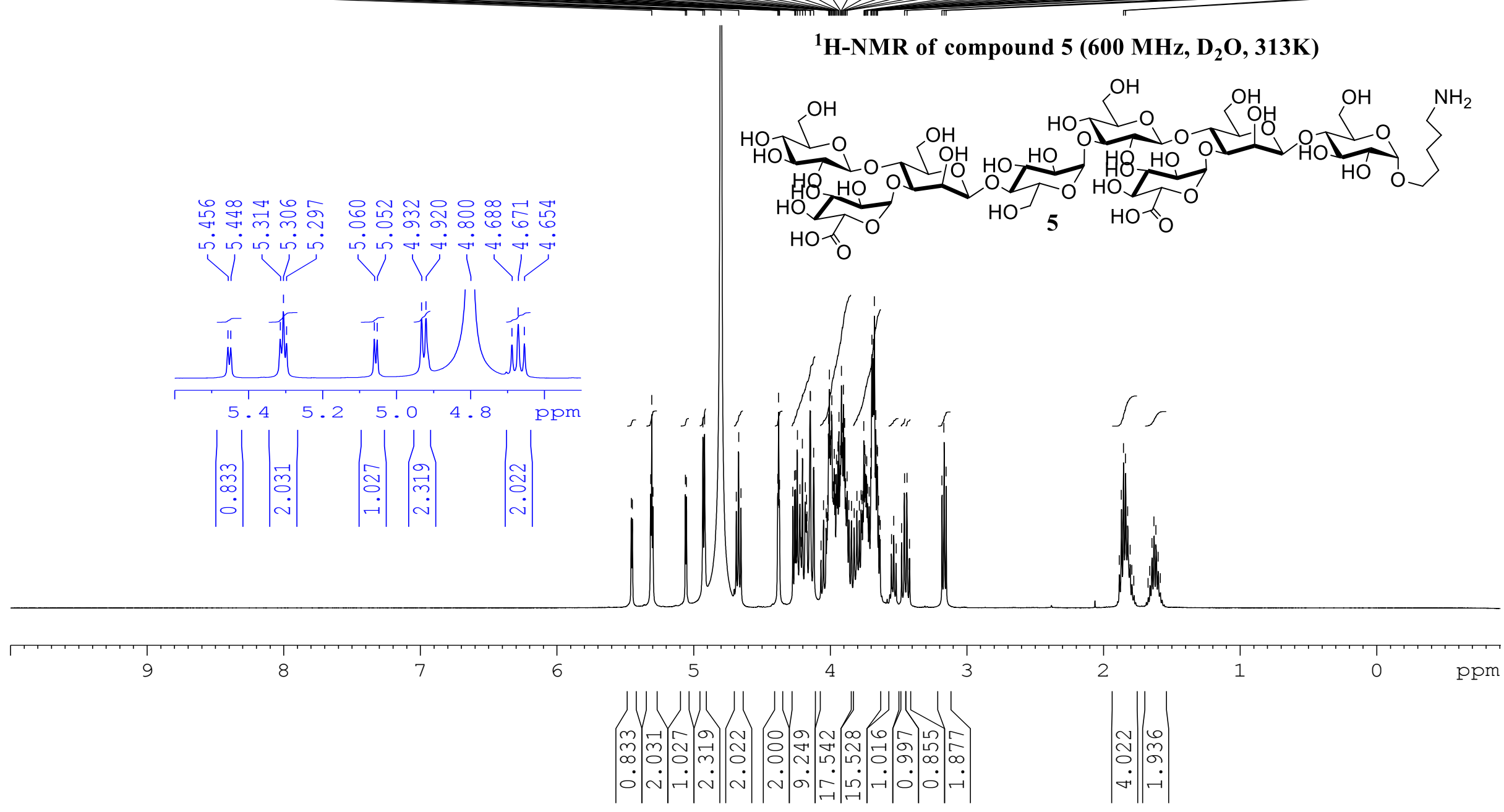




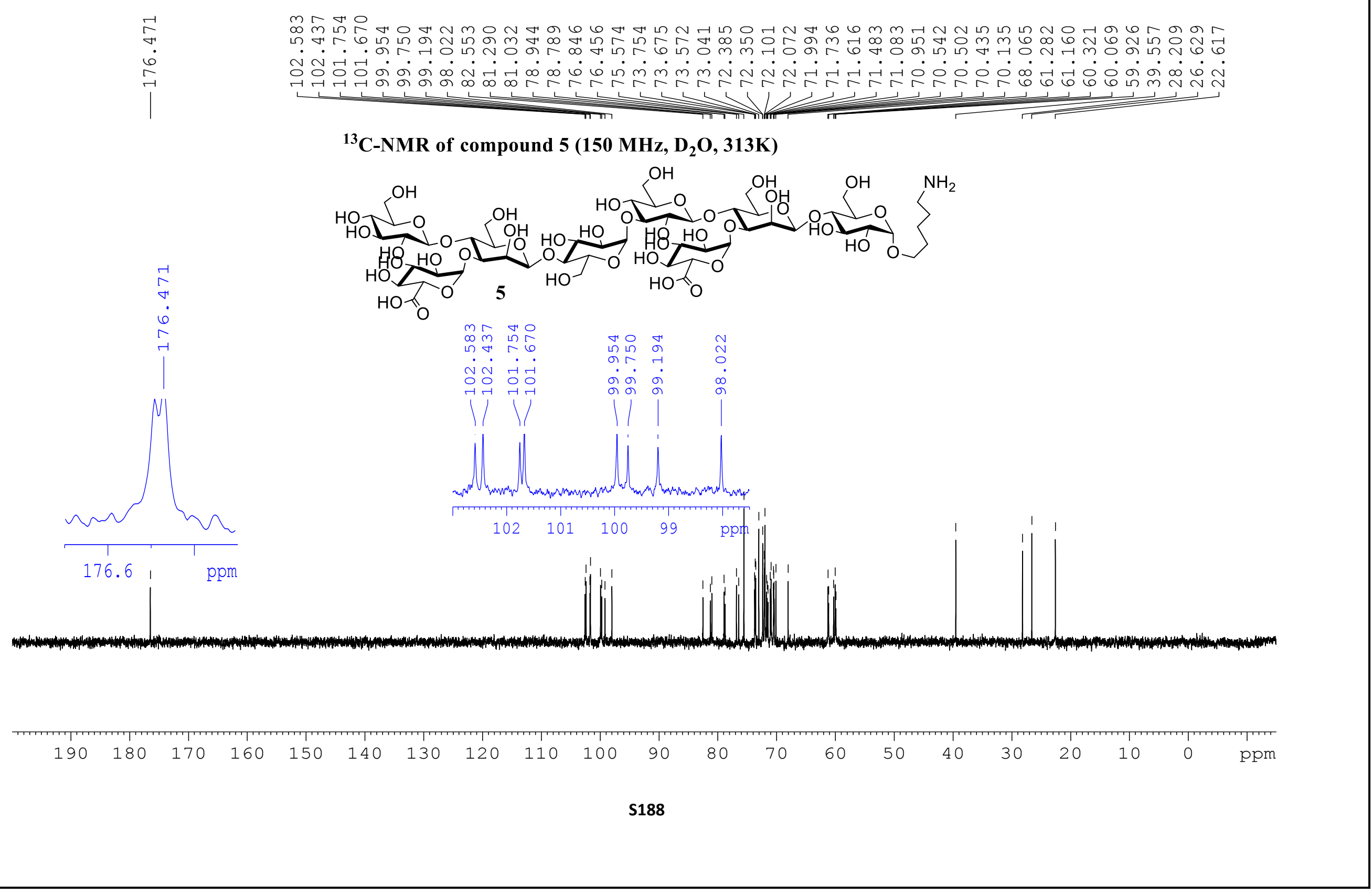




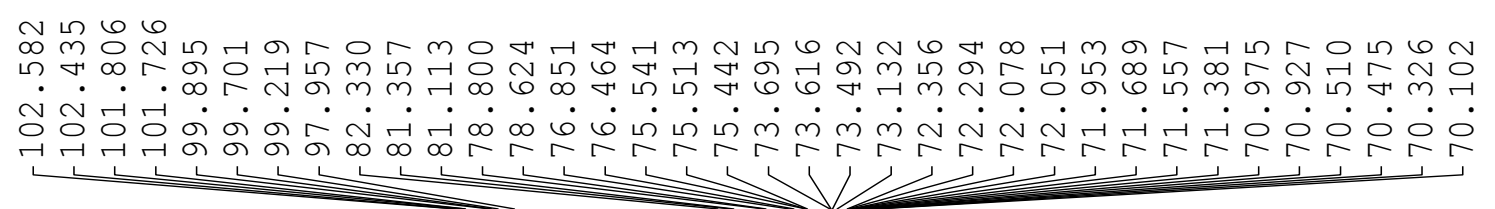

DEPT 135 NMR of compound 5 (150 MHz, $\left.\mathrm{D}_{2} \mathrm{O}, 313 \mathrm{~K}\right)$

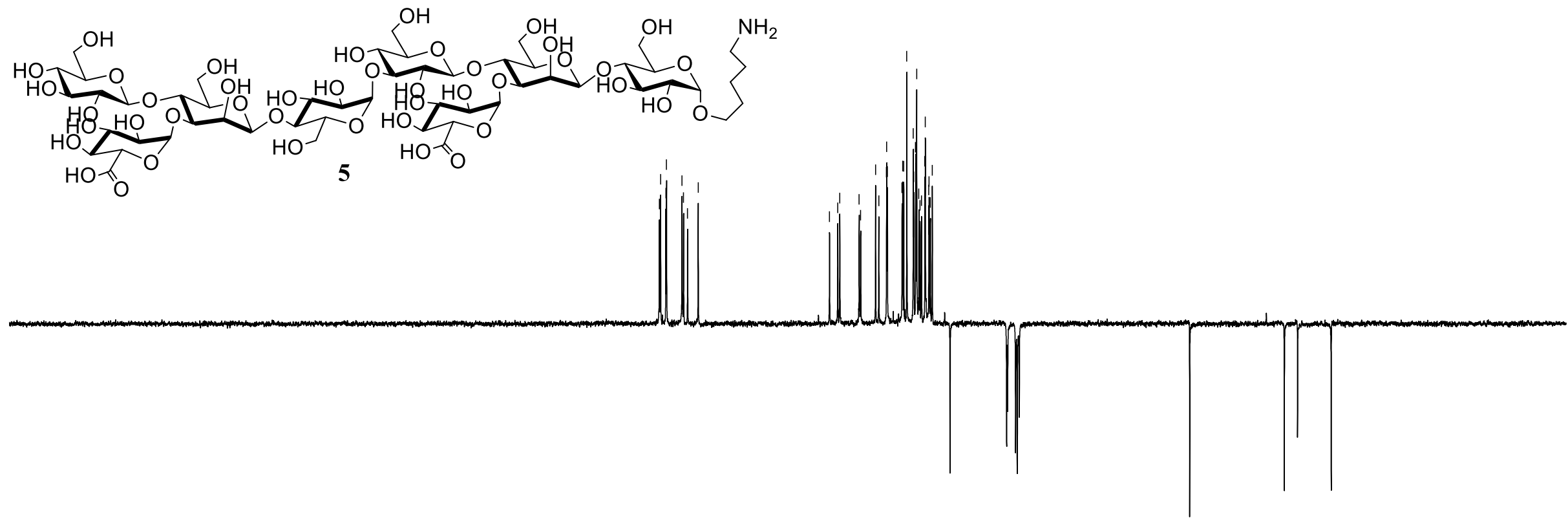




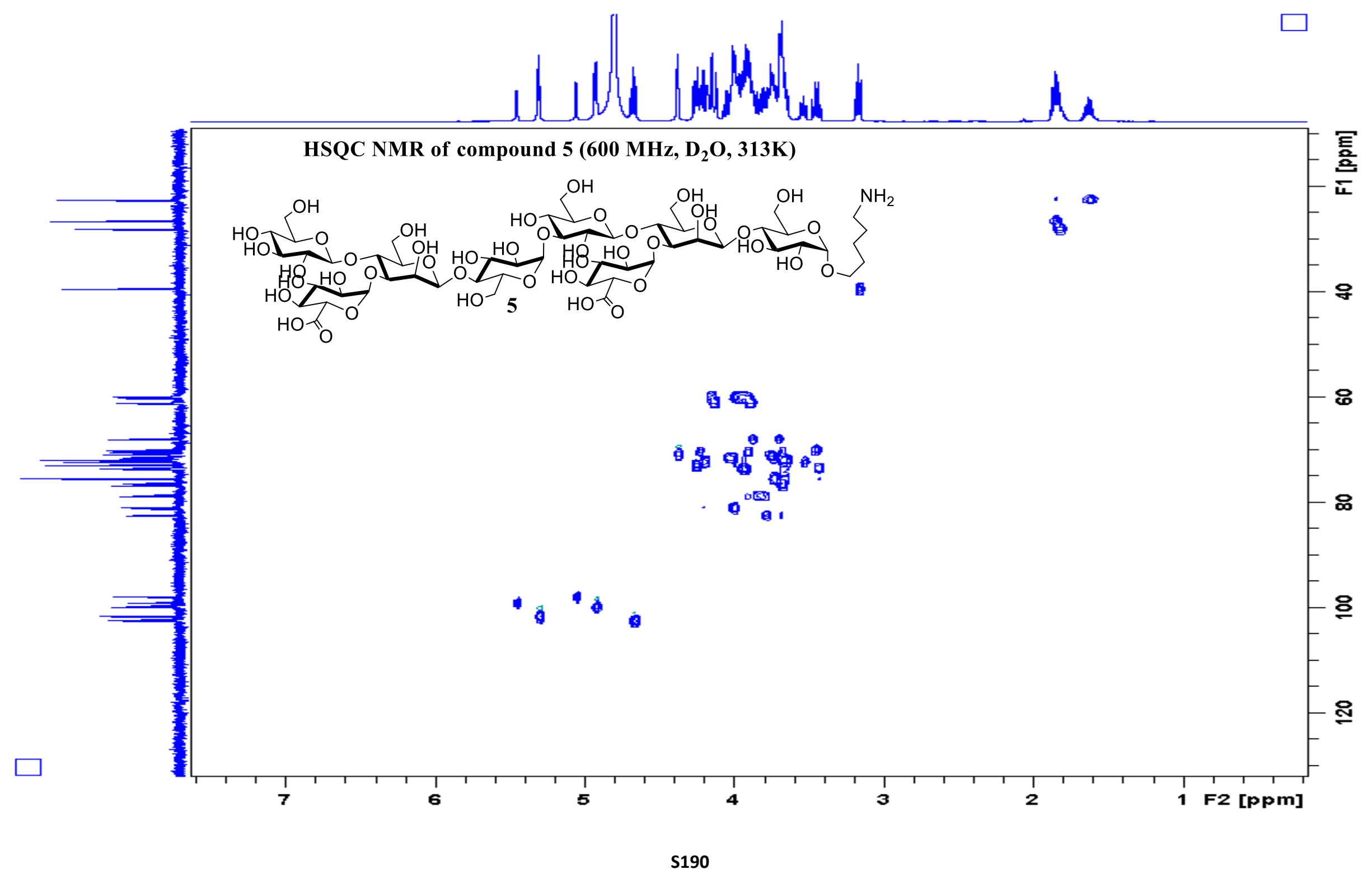




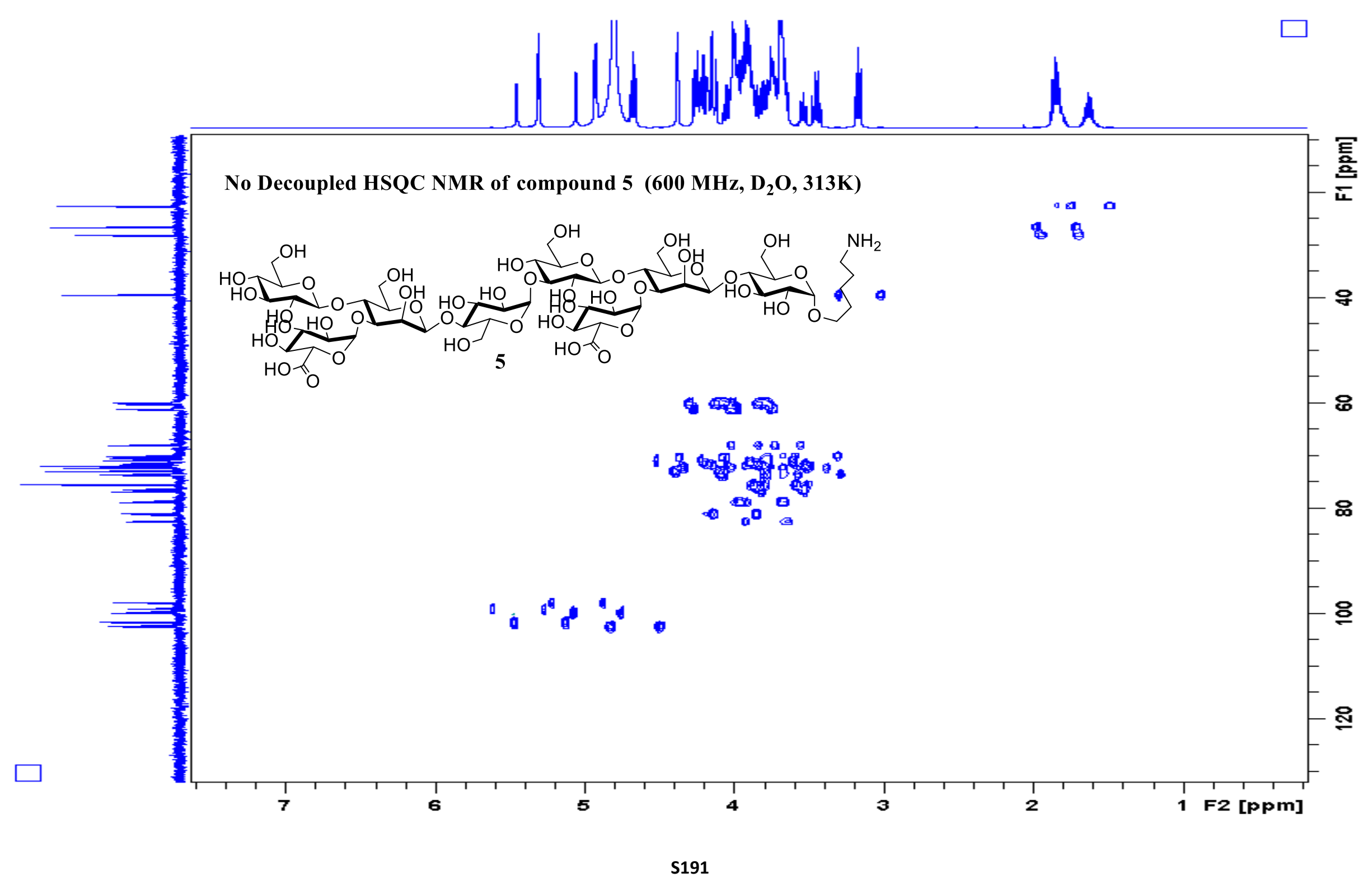




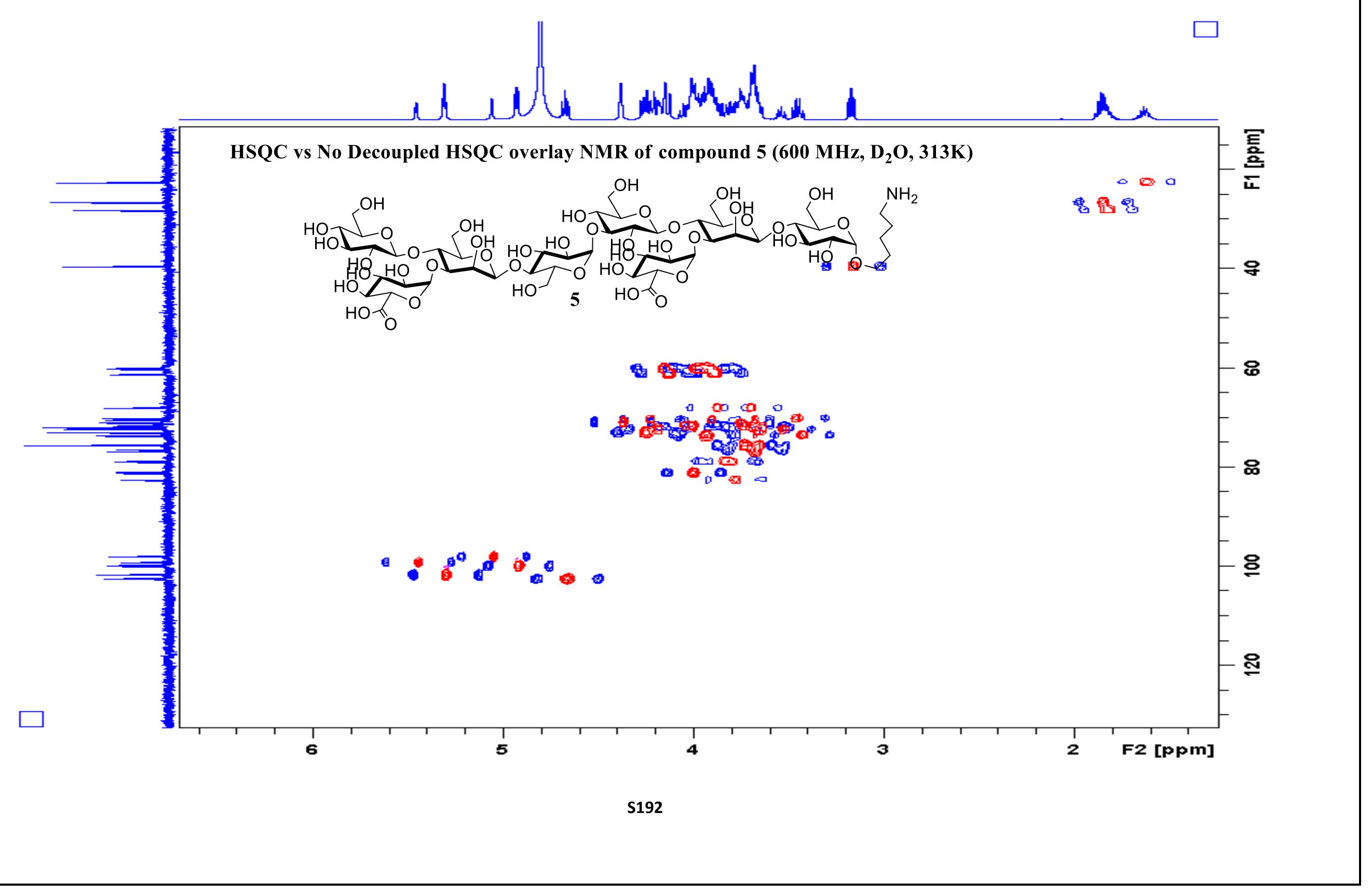




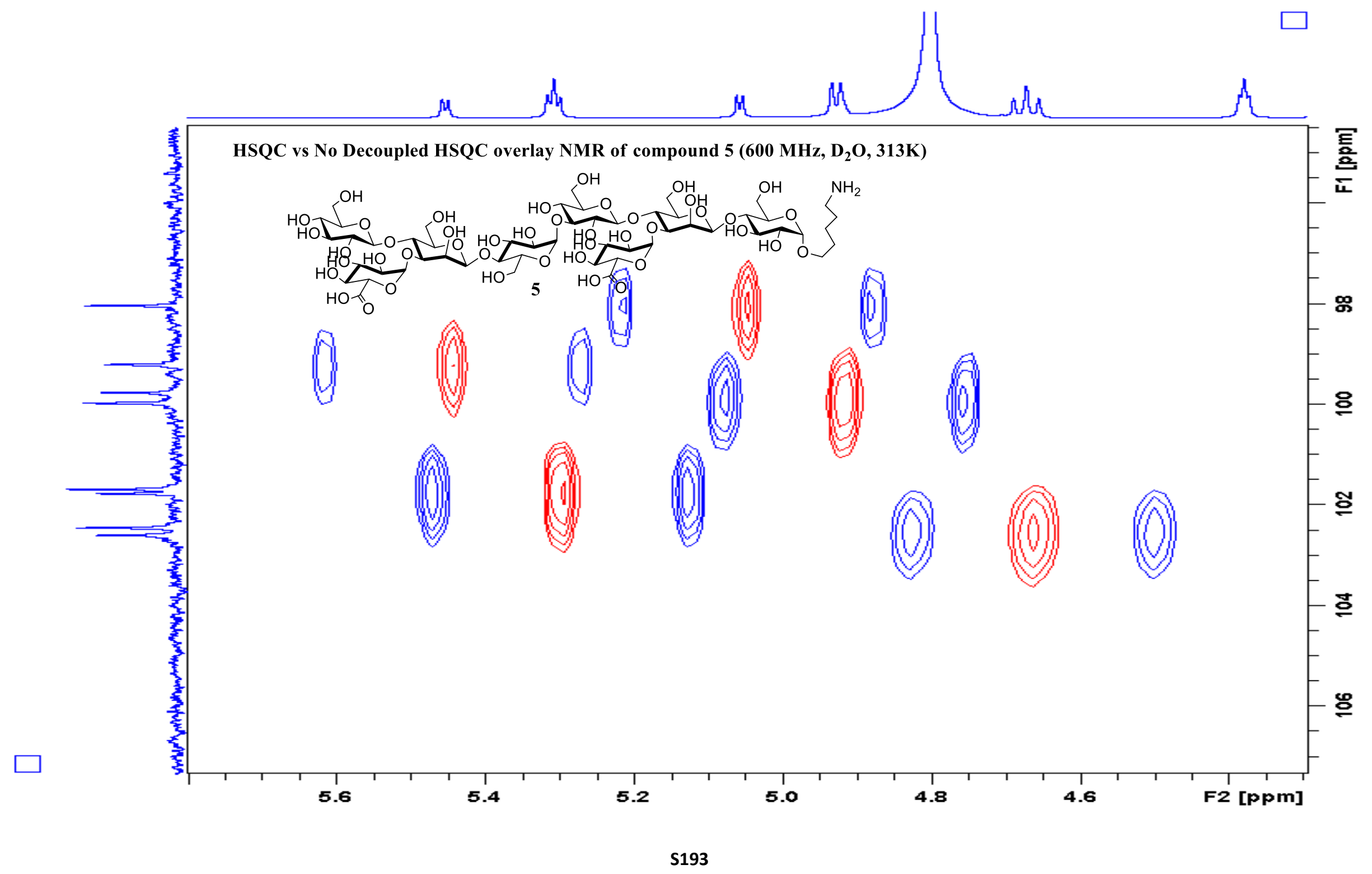




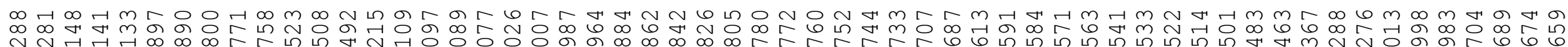

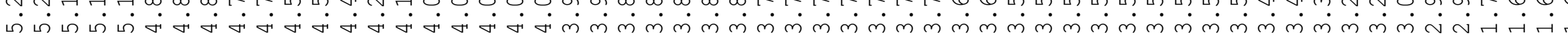

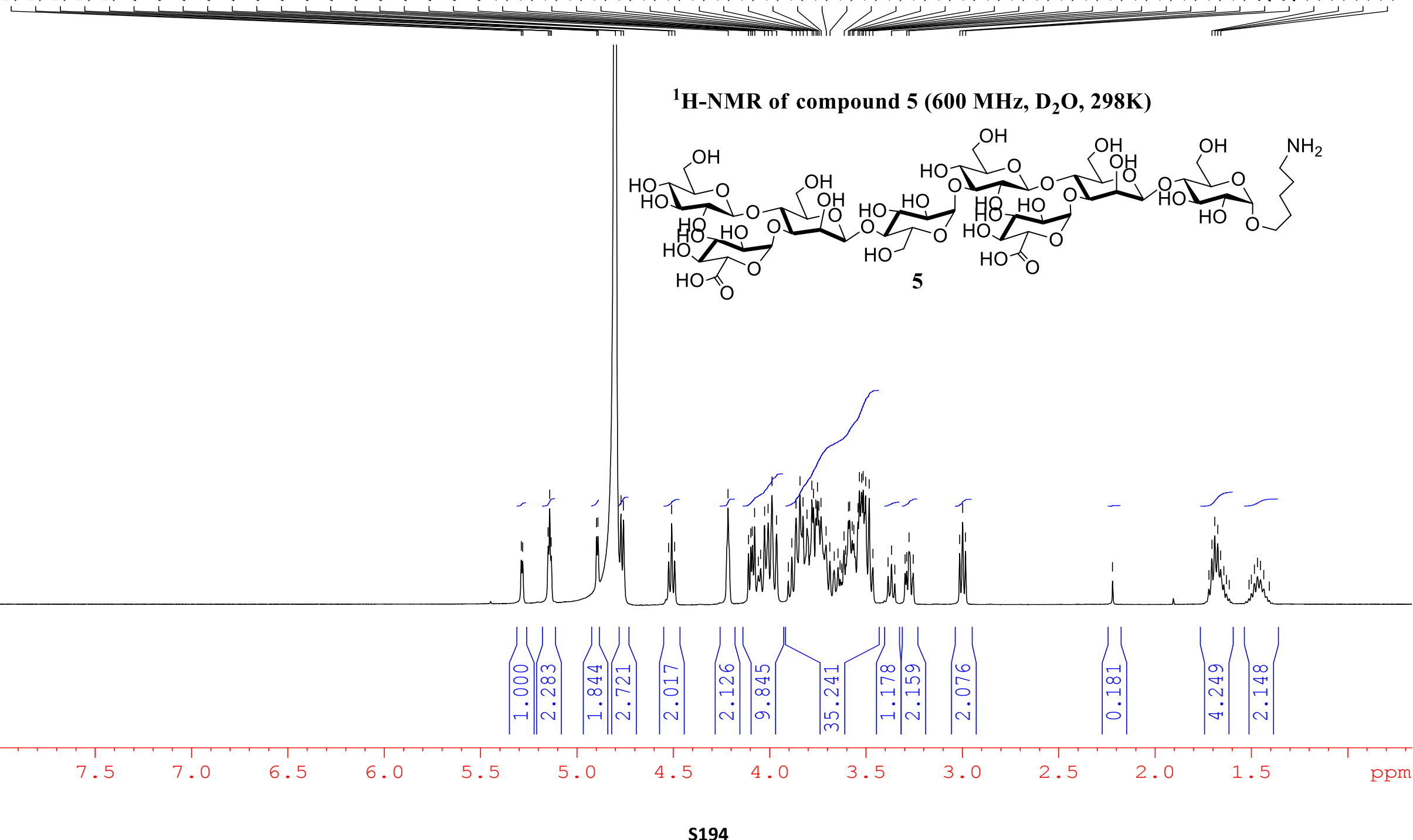




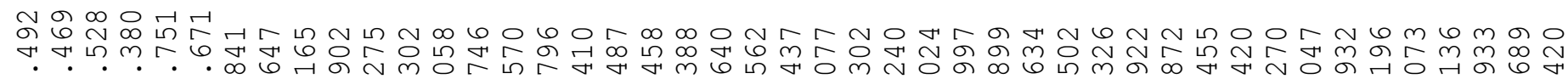

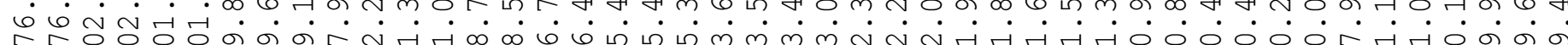
국가국의

\section{${ }^{13} \mathrm{C}-\mathrm{NMR}$ of compound 5 (150 MHz, $\left.\mathrm{D}_{2} \mathrm{O}, 298 \mathrm{~K}\right)$}

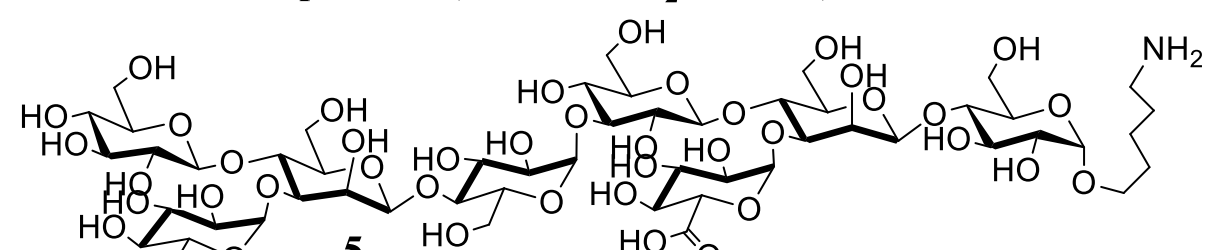

$\mathrm{HO}$

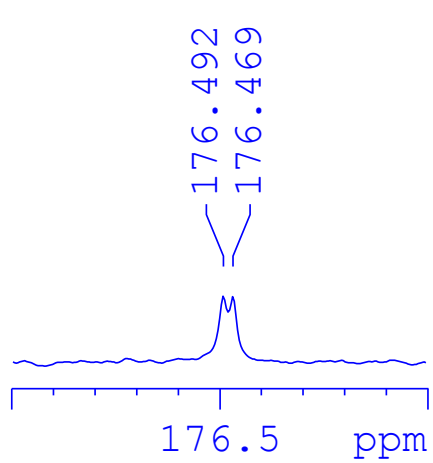

$\infty$ 다 다

N $\infty$ i

n m

$\cdot \cdot \cdot \infty 6$ -

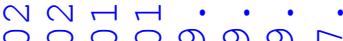

更

$1 / 1$
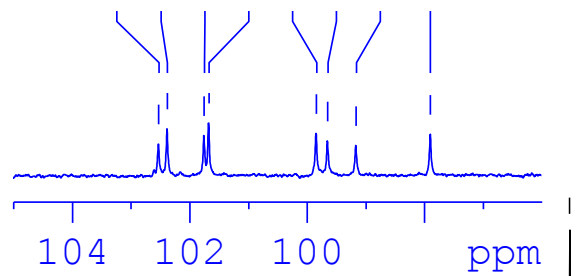
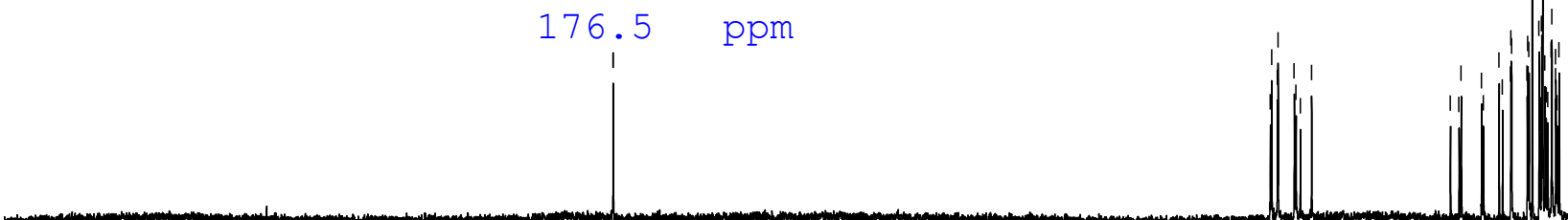


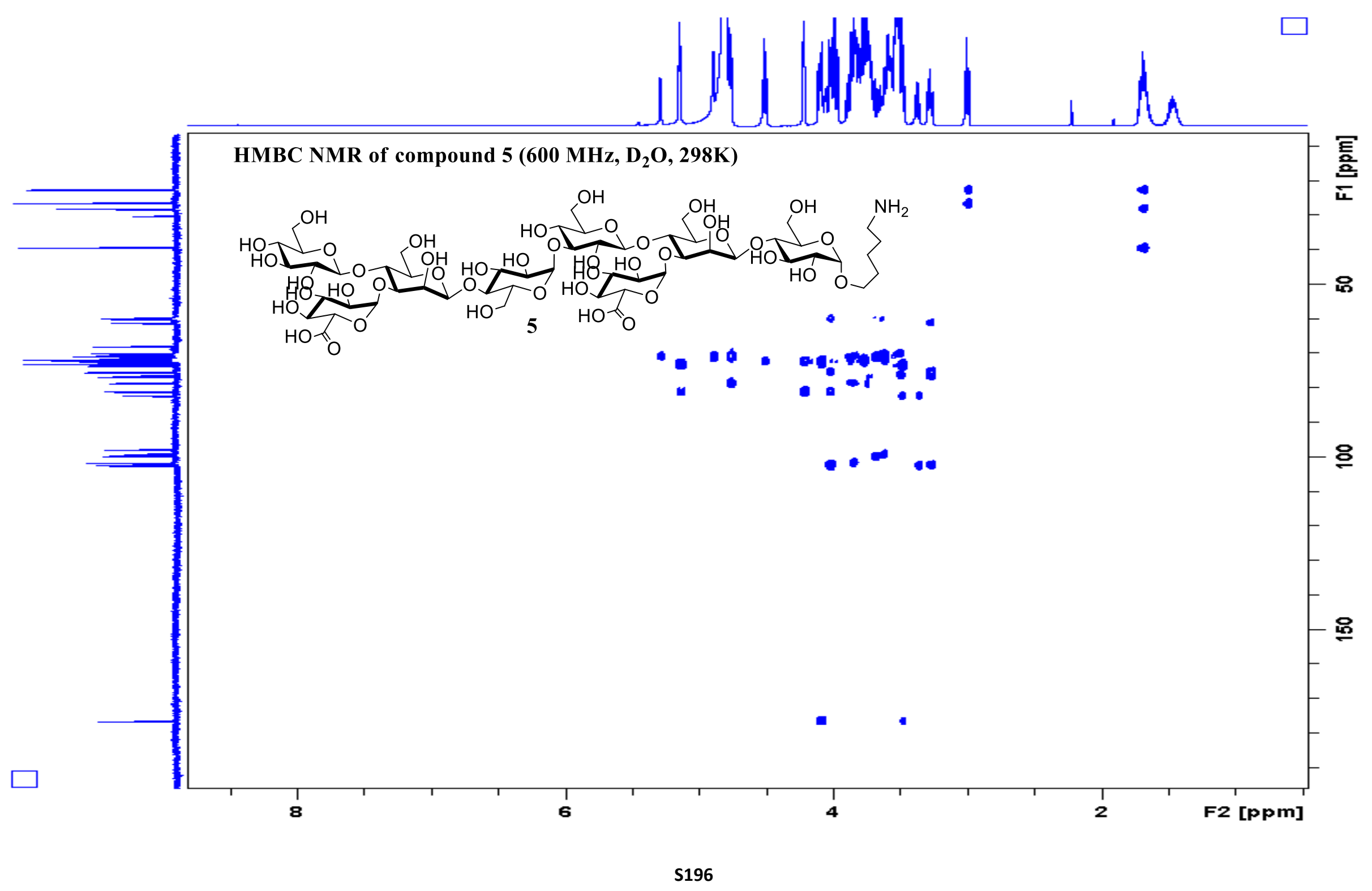




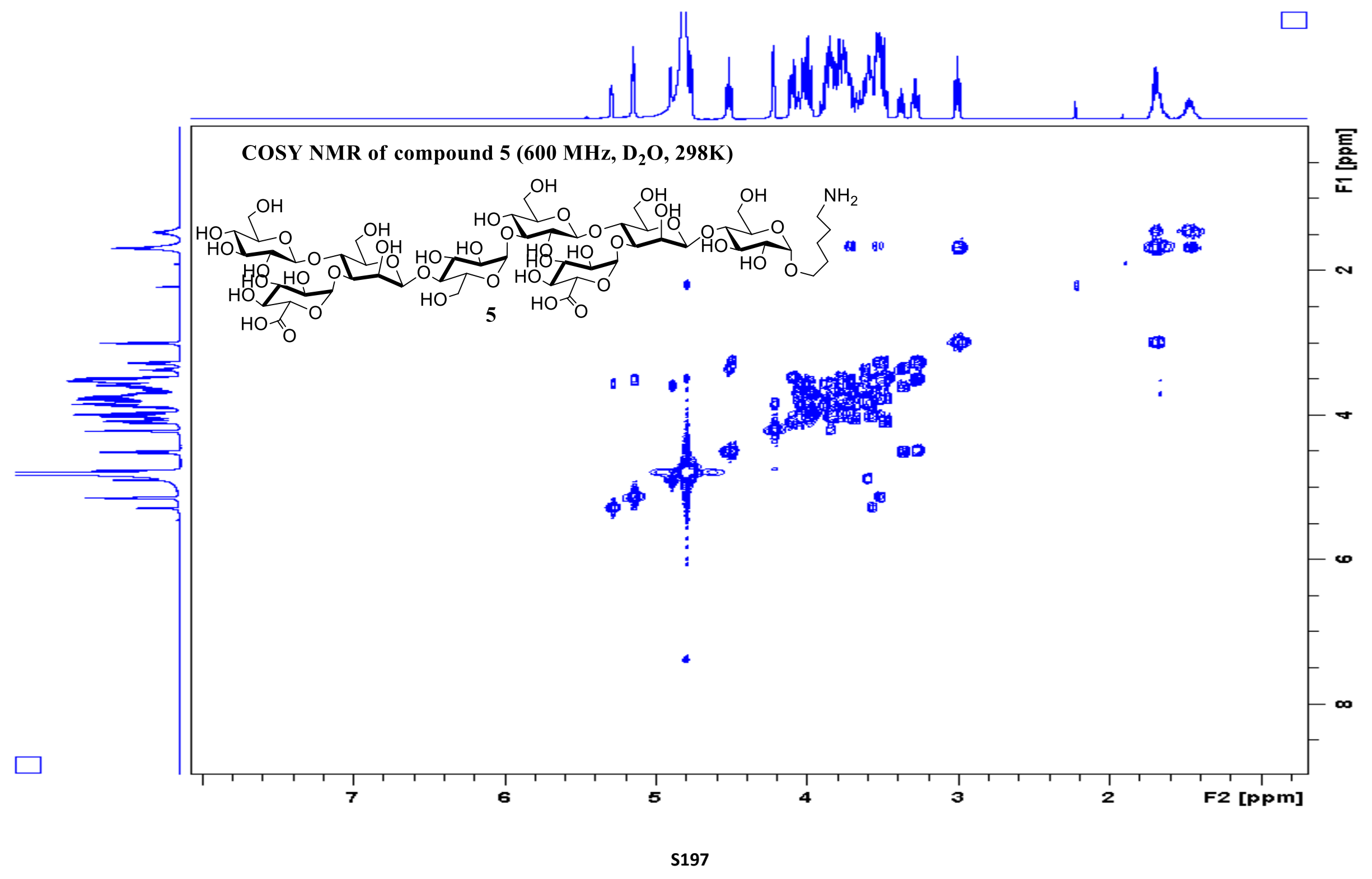




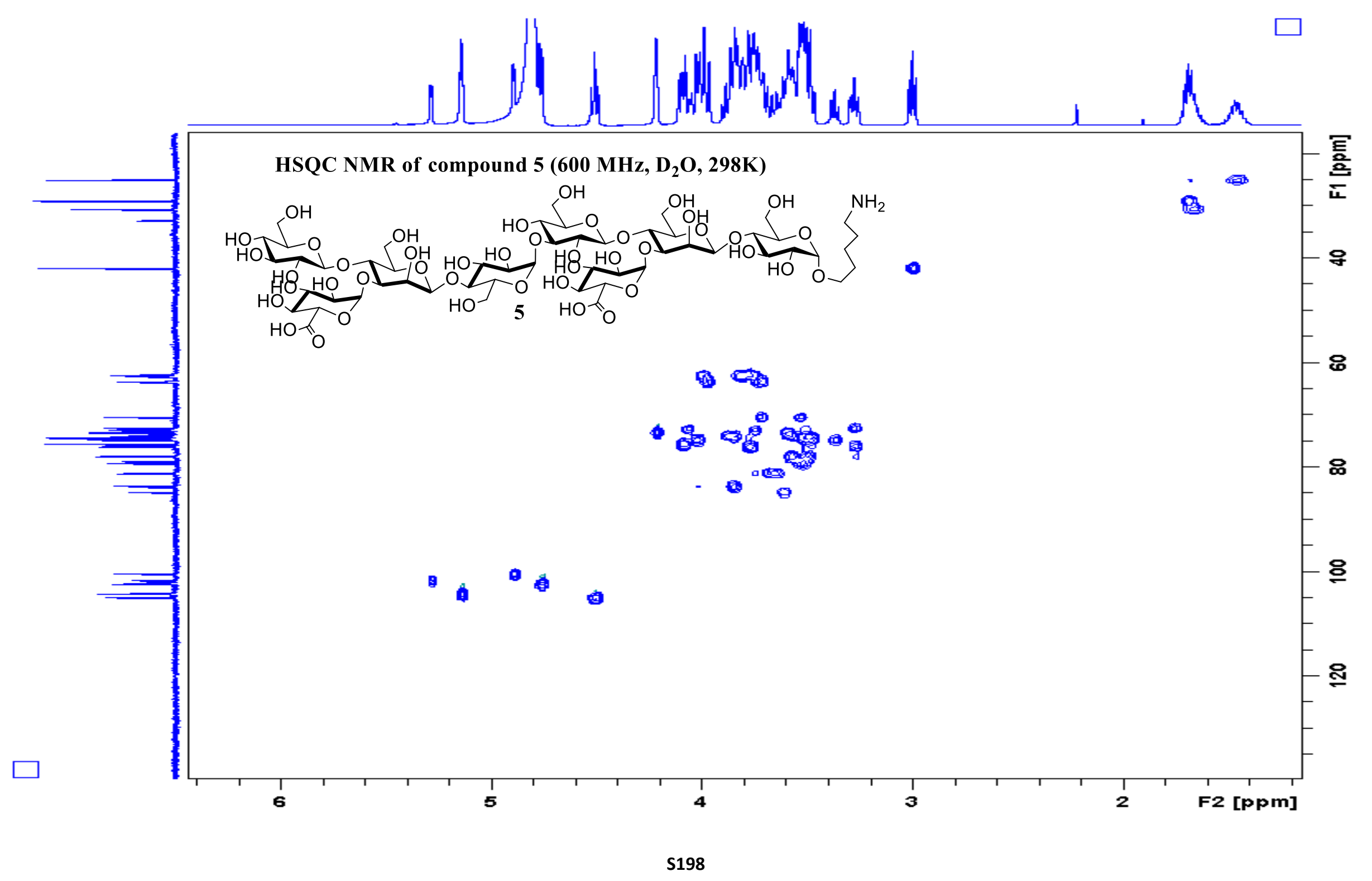




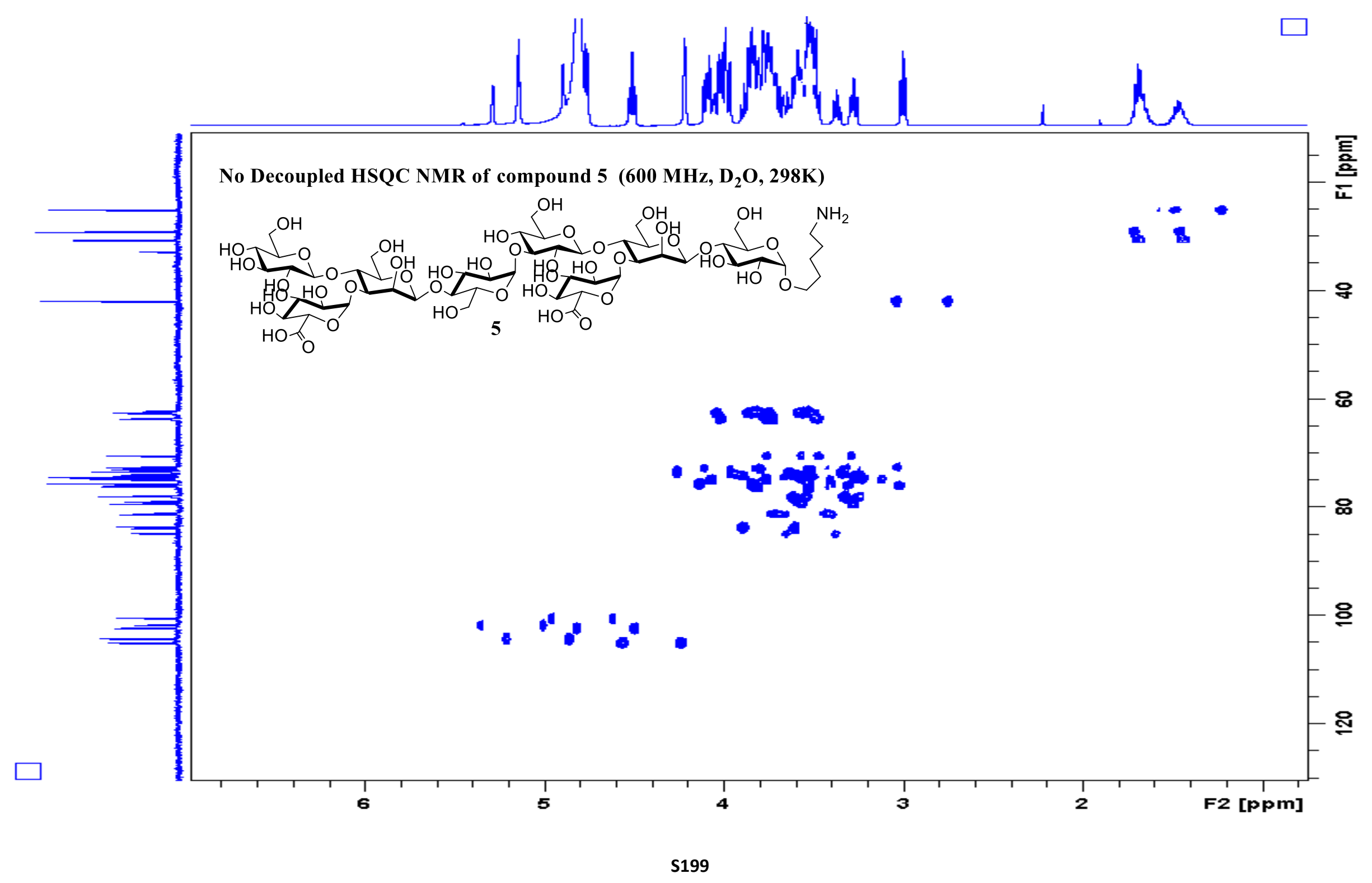




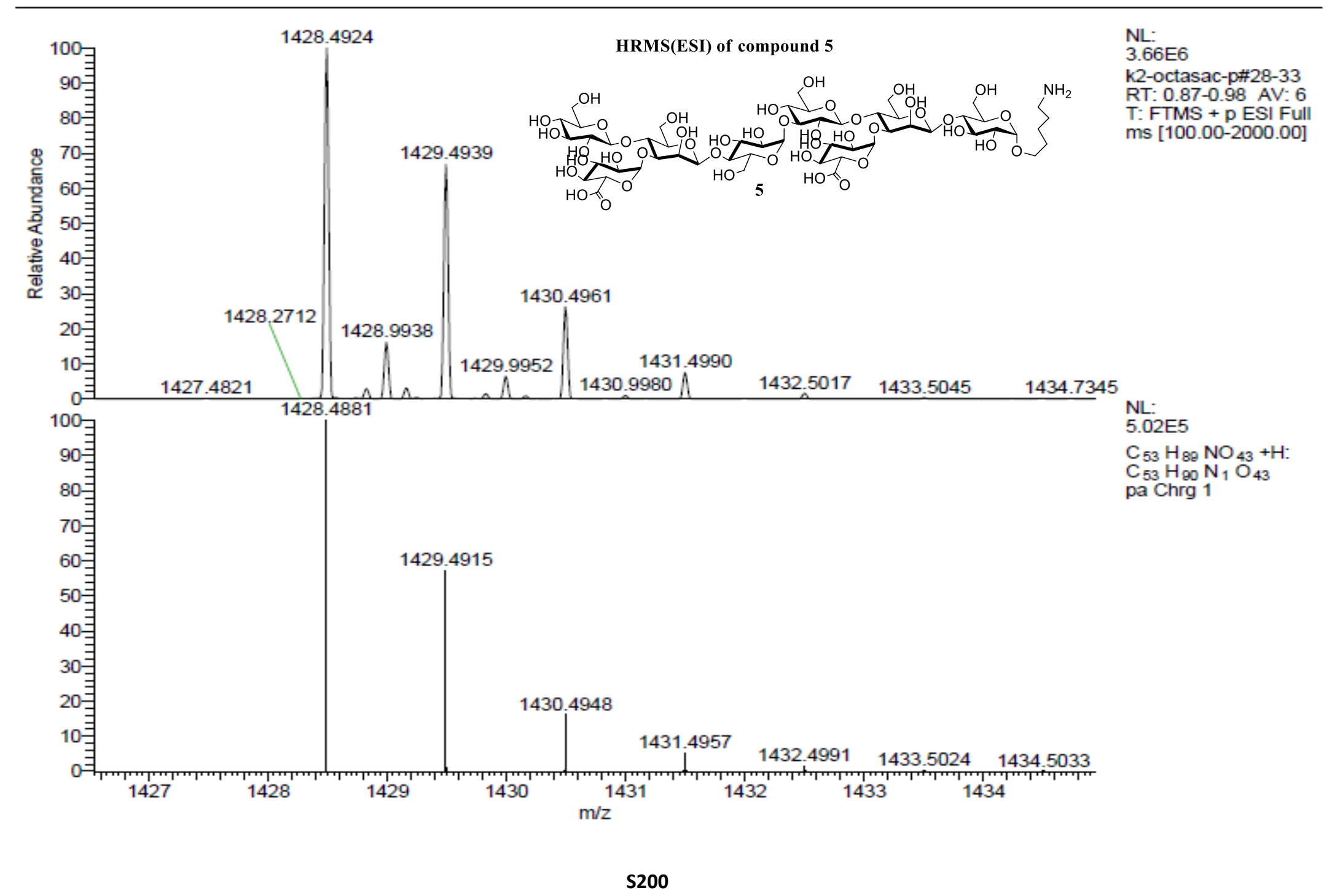




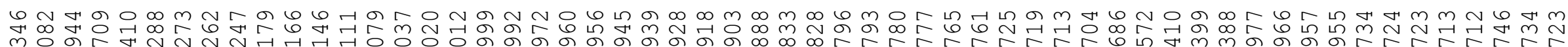

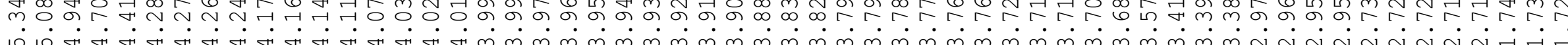

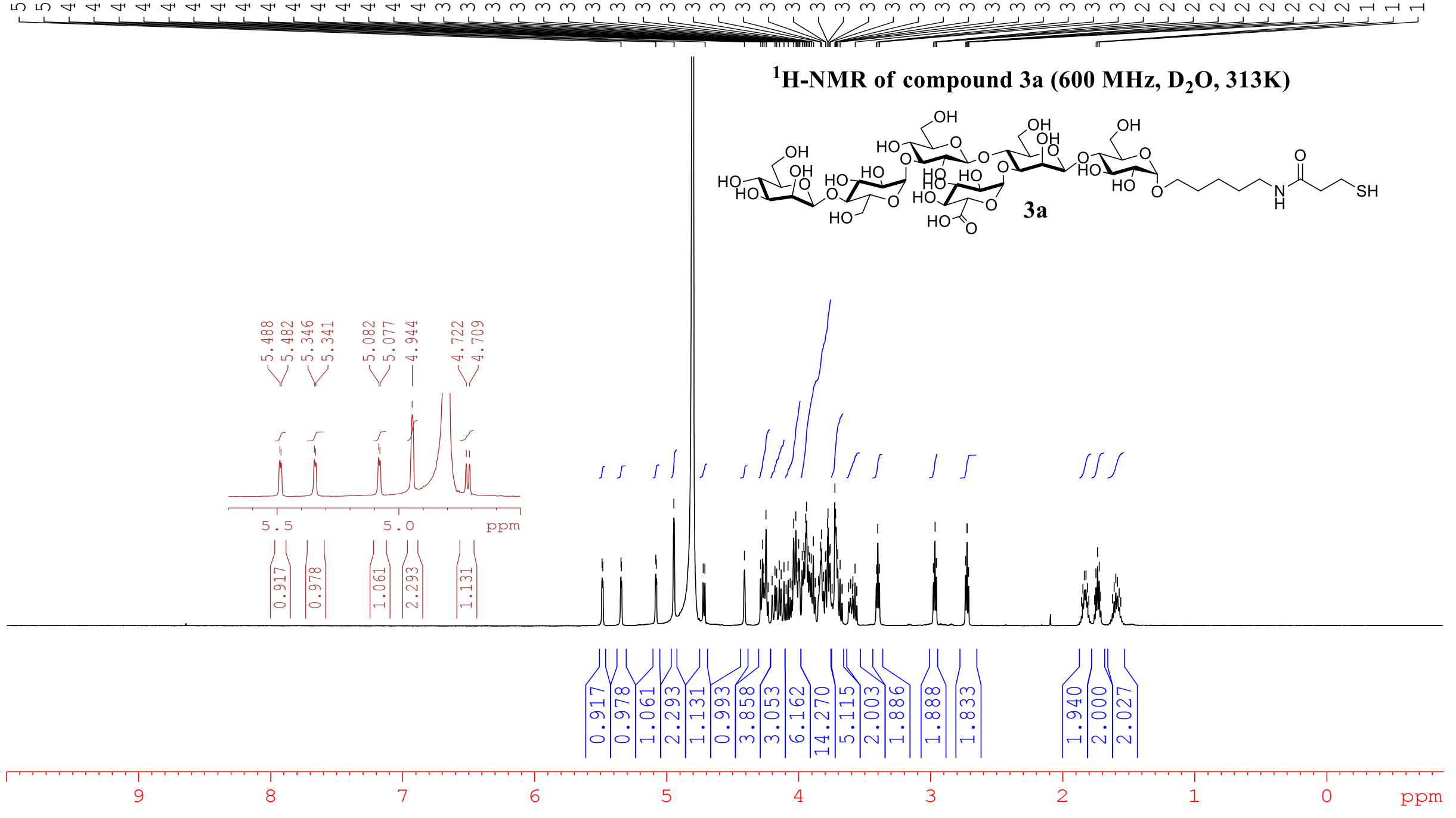




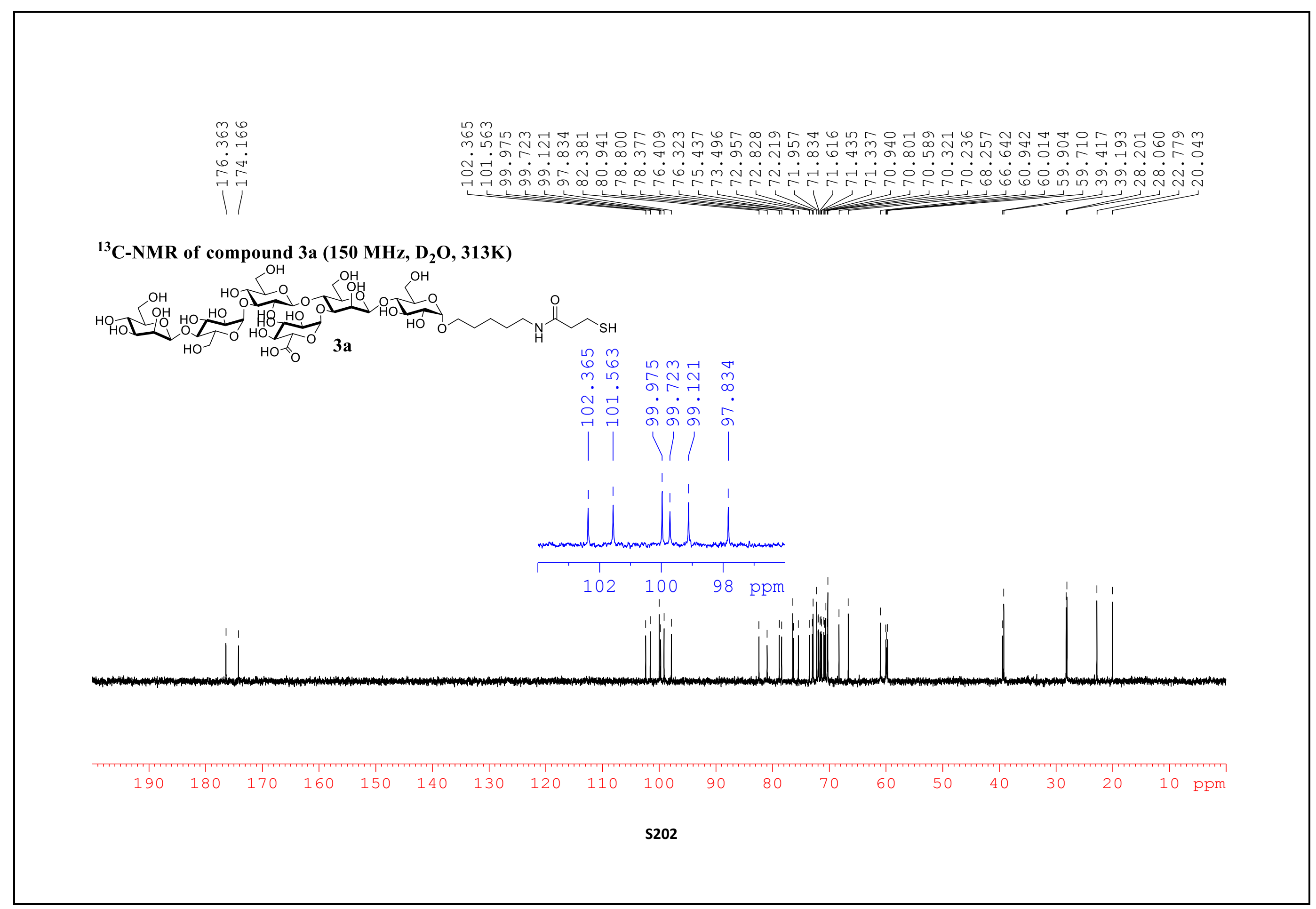




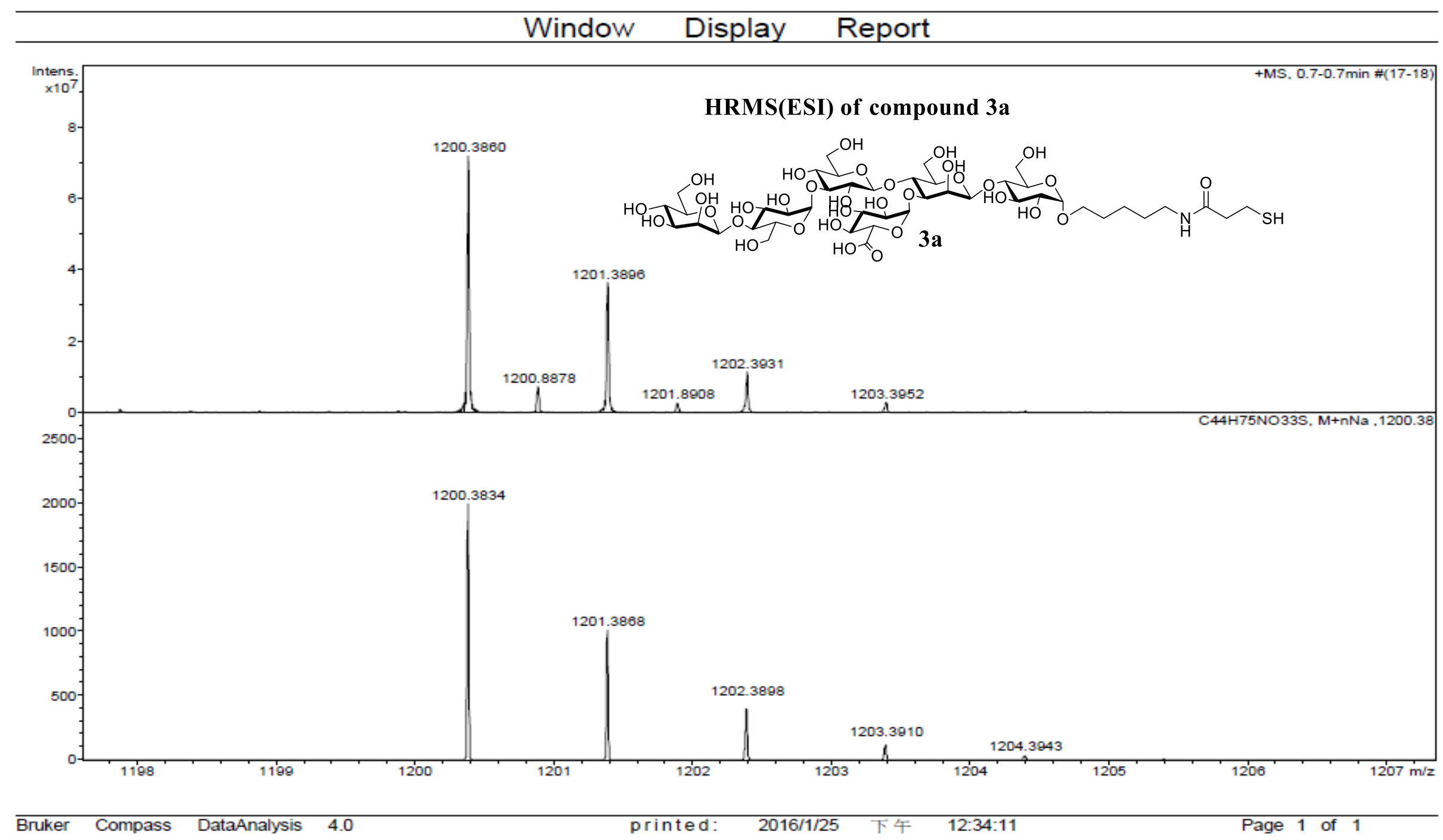




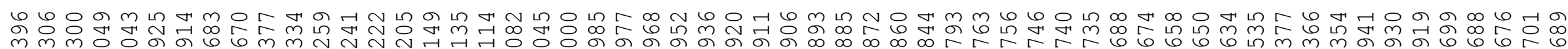

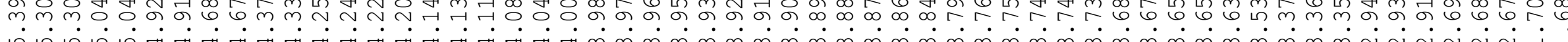

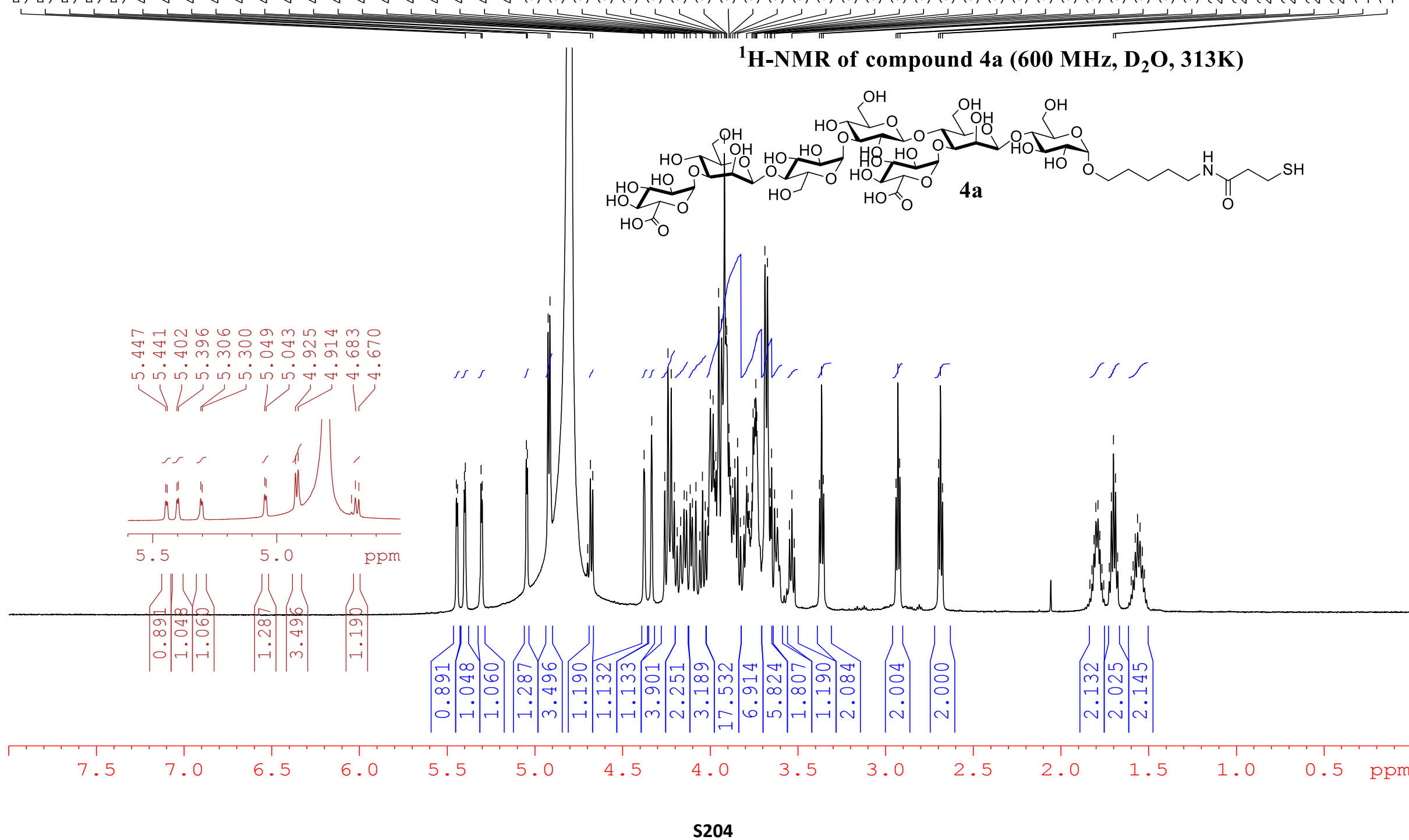




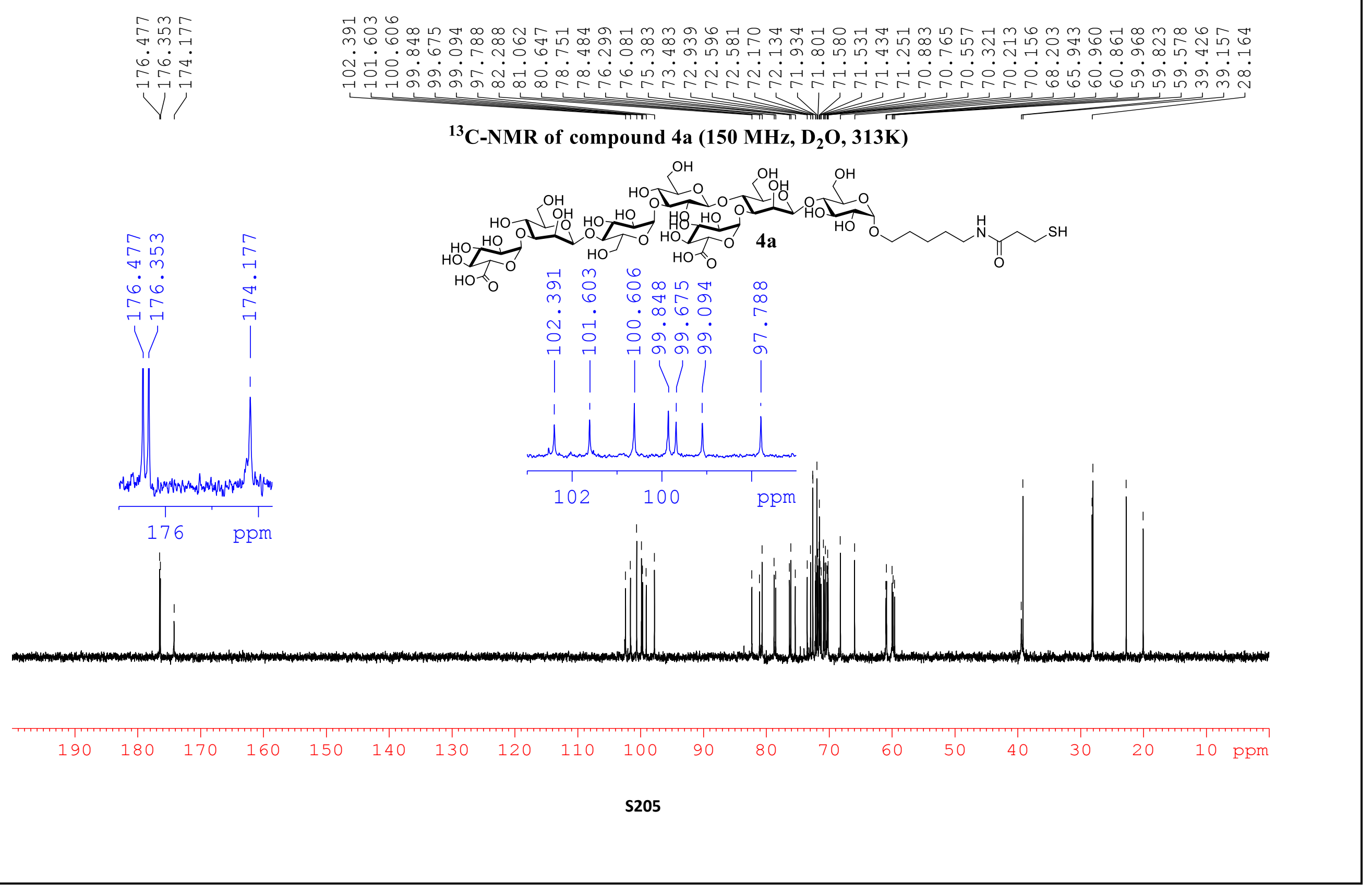




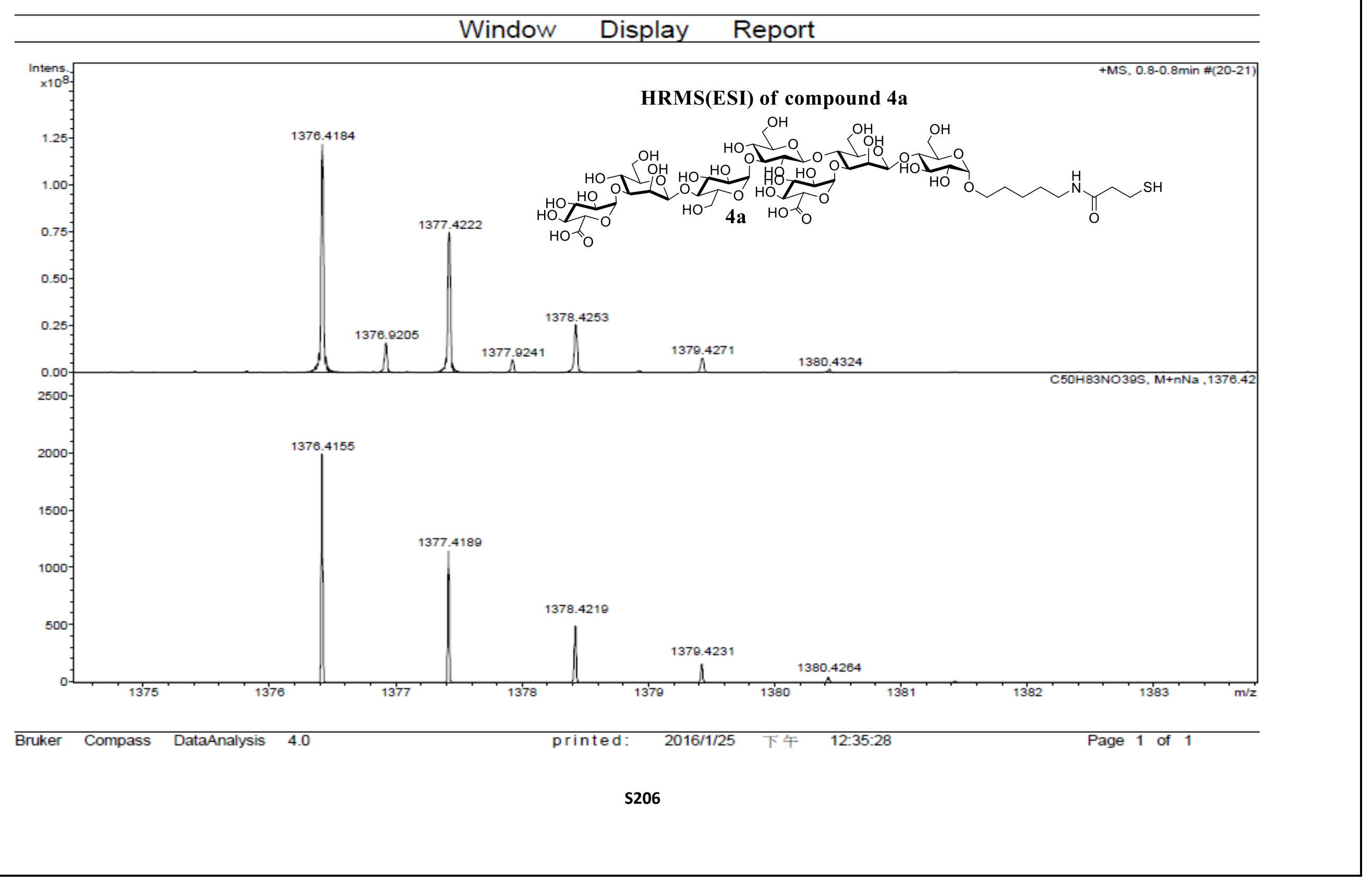




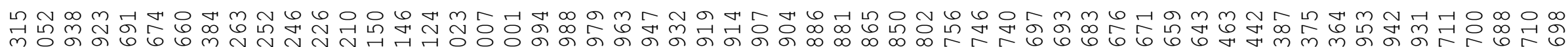

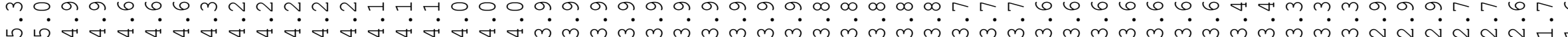

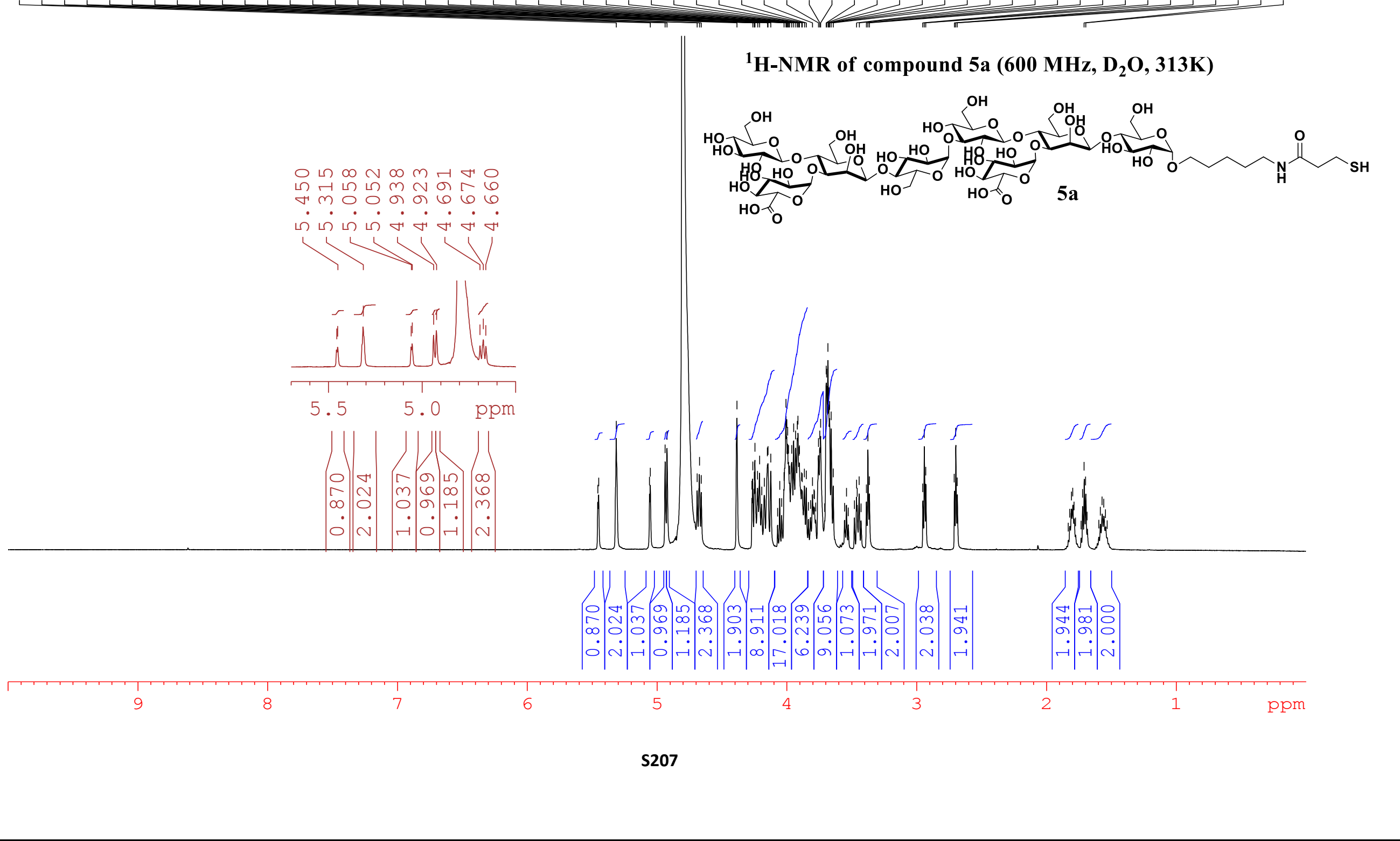




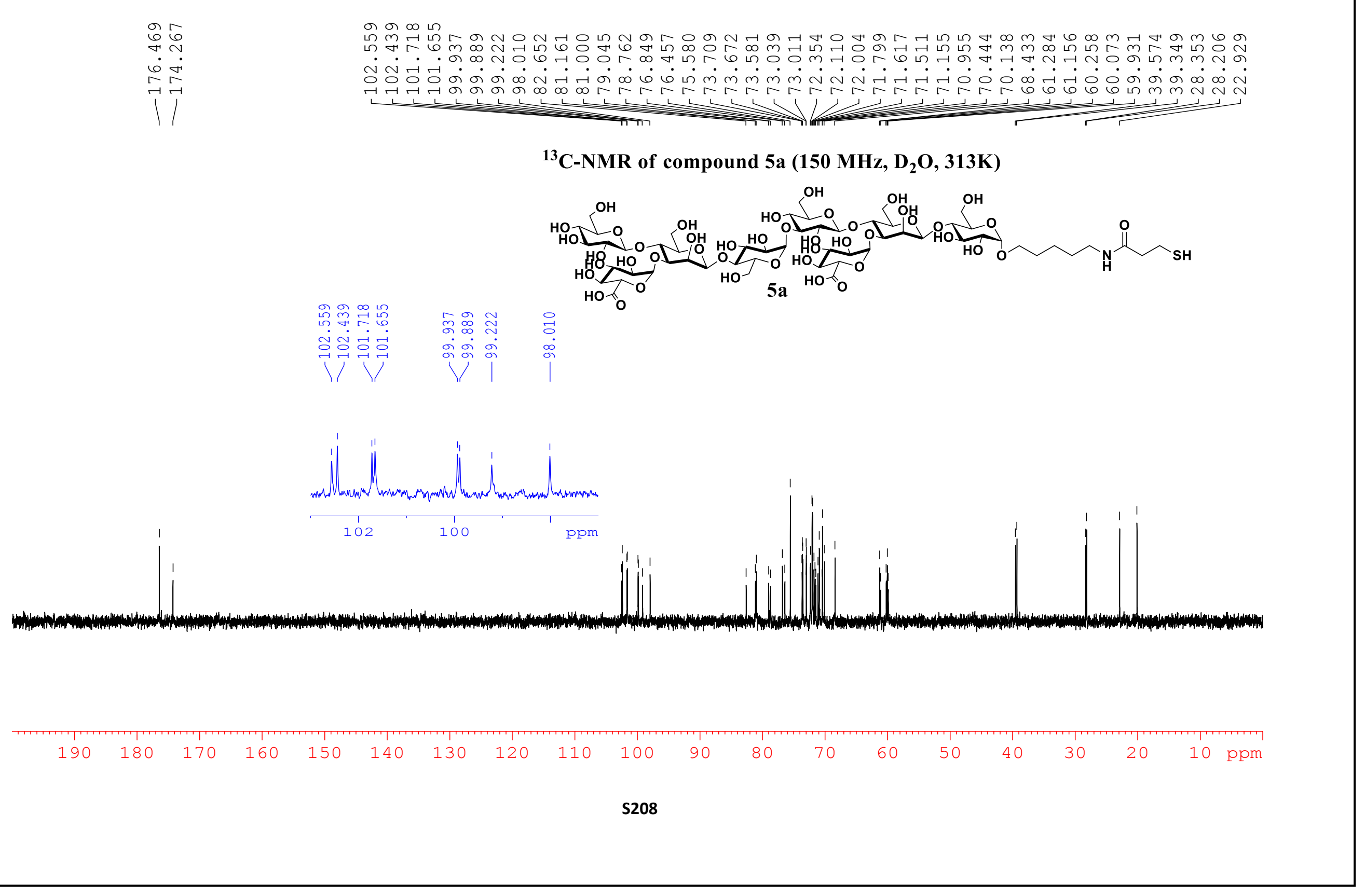




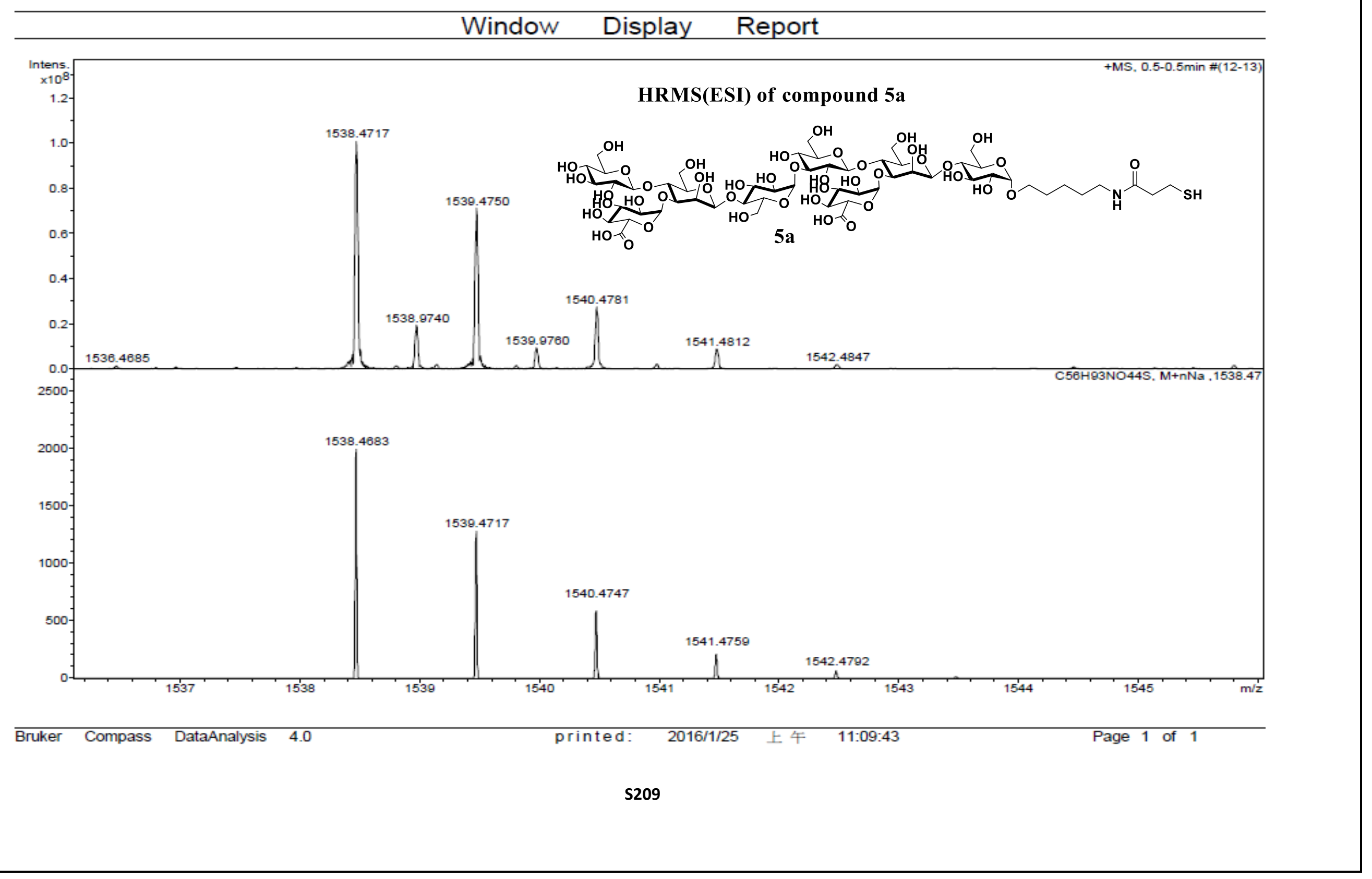

